UNIVERSIDAD POLITÉCNICA DE MADRID

ESCUELA TÉCNICA SUPERIOR DE INGENIEROS INDUSTRIALES DEPARTAMENTO DE INGENIERÍA MECÁNICA

\title{
Variable-stiffness joints with embedded force sensor for high-performance wearable gait exoskeletons
}

\author{
DOCTORAL THESIS \\ Manuel Javier Cestari Soto \\ MSc. Mechanical Engineering.
}




\title{
Variable-stiffness joints with embedded force sensor for high-performance wearable gait exoskeletons
}

\author{
Author \\ Manuel Javier Cestari Soto \\ MSc. Mechanical Engineer \\ Directors \\ Dr. Elena García Armada \\ $\mathrm{PhD}$ Robotics and Automation, Industrial Engineer \\ Dr. Andrés Díaz Lantada \\ $\mathrm{PhD}$ Industrial Engineer
}

2016 
Título: Variable-stiffness joints with embedded force sensor for high-performance wearable gait exoskeletons

Autor: Manuel Javier Cestari Soto

Tribunal nombrado por el Mgfco. y Excmo. Sr. Rector de la Universidad Politécnica de Madrid, el día de de 2016

Presidente:

Secretario:

Vocal:

Vocal:

Vocal:

Suplente:

Suplente:

Realizado el acto de lectura y defensa de la tesis el día de de 2016, en , el tribunal acuerda otorgar la calificación de: 
To Grey, my parents, and brothers.- 


\section{Acknowledgments}

I would like to thank my promotor Elena who gave me the opportunity to work towards this $\mathrm{PhD}$, trusting and encouraging my work. I would also like to particularly thank Dani and Juan C. with who I have shared this journey.

To Andrés, for all his assistance and representation before the University.

I would also like to thank all the help from the staff of the CAR, the administrative personnel and the guys from the workshop.

Special thanks to my colleagues who from one way or another have been involved in the development of this work: Jorge, Luis, Javi, and Mariano. Particularly to Gabriel for allowing me his $3 \mathrm{~d}$ printer for several test parts.

To my family, parents, brothers, and wife from whom I always felt supported. 


\section{Abstract}

The growing field of exoskeletons and wearable devices for walking assistance and rehabilitation has advanced considerably over the past few years. The current use of commercial devices is in-hospital rehabilitation of spinal cord injured, nevertheless the purpose of this technology is challenging: to provide gait assistance in daily life activities to the broadest segment of neurological disorders affecting walking and balance. A number of difficulties make this goal a challenge, but to name a few: (1) The degenerative character of the majority of neurological disorders and the very complex symptomatology associated to the disease requires an intelligent exoskeleton able to self-adapt to the disease; (2) The assistance to daily life activities requires a gait exoskeleton for particular use provided with energy autonomy for -at least- a journey. The causes for current commercial devices for not approaching this capabilities are mostly related to the nature and control approach of the joint actuation systems. Currently, commercial devices contain joints with stiff actuators that cannot adapt to unpredictable environments. These actuators consume more energy and may not be appropriate for human-machine interactions. Adjustable compliant actuators are being designed and implemented because of their ability to minimize large forces due to shocks, to safely interact with the user, and their ability to store and release energy in passive elastic elements. Many simulation-based researches have been performed evaluating the benefits of incorporating compliant joints in robotic exoskeletons. Spasticity and spasmodic movements are common between patients with motor deficiencies; and it comes from this research that compliant actuators could efficiently absorb these perturbations and improve the joints control.

This $\mathrm{PhD}$ thesis aims at advancing beyond the State of the Art in joint actuation systems for gait exoskeletons with the purposes of: enabling joint adaptation to variable symptomatology and improving energy efficiency, and adaptability during walking. By analyzing the biomechanics of locomotion, the characteristics and requirements of the main joints involved in the dynamic locomotion cycle are identified and analyzed. This doctoral work presents the design and development of two novel compliant actuators intended to fulfill the requirements for actuating 
joint exoskeletons. The main feature of the novel systems is that the compliant elements simultaneously allow measuring of the torque exerted by the joint.

Conceived as force-controlled compliant actuators, these actuators with Adjustable Rigidity and Embedded Sensor, ARES and ARES-XL are intended to be implemented in the joints of the ATLAS pediatric exoskeleton. The resulting device is a force controlled-compliant exoskeleton for children with neuromuscular diseases which allow the exploitation of the intrinsic dynamic during the locomotion cycle. ARES capabilities are presented and evaluated, proving its torque tracking capabilities at different stiffness levels.

The versatile operation of the joints such as the knee, could be emulated, and exploited by providing the elements that can control the use of the energy stored in the appropriate phases of the gait. ARES-XL allows the implementation of an add-on locking mechanism to this system, in combination with its zero stiffness capability and large deflection range. The evaluation of the system proves how this design exceeds the main capabilities of the original realization, as well as providing versatile actuation that could lead to its implementation in multiple joints.

During this work an assessment of the compliant exoskeleton was performed by walking under certain constrains. Comparing the behavior of the joints under different stiffness conditions, the inherent compliant of the presented actuators showed natural adaptability during the gait cycle, and regions of shock absorption.

The work developed in this $\mathrm{PhD}$ thesis is expected to continue being implemented in exoskeleton, and robotic prosthetics applications in a research and commercial level. Several publications in relevant journals, and international conferences have been published as a consequence of the research performed during this $\mathrm{PhD}$ work. There are currently three patents product of this research, they are being commercially exploited by a SME specialized on robotics for healthcare. Future works will focused in the optimization of the size and weight of the compliant systems, combined with the development and implementation of control strategies adapted to the specific users and environment conditions, for energy efficiency and more natural gaits. 


\section{Resumen}

El creciente campo de exoesqueletos y dispositivos portátiles de asistencia al caminado y rehabilitación ha avanzado considerablemente en los últimos años. Hoy en día, el uso actual de los dispositivos comerciales se centra en la rehabilitación de lesionados medulares en el hospital, sin embargo, el propósito retador de esta tecnología: proporcionar asistencia a la marcha en las actividades de la vida diaria al amplio grupo de trastornos neurológicos que afectan al caminar y el equilibrio. Un número de dificultades hacen que este objetivo un desafío, para nombrar unos pocos: (1) El carácter degenerativa de la mayoría de los trastornos neurológicos y la muy compleja sintomatología asociada a la enfermedad requiere un exoesqueleto inteligente capaz de autoadaptarse a la patología; (2) La asistencia a las actividades de la vida diaria requiere un exoesqueleto de marcha para su uso particular, siempre con la autonomía energética para -al menos- un viaje. Las razones por las que los dispositivos comerciales actuales no se acercan a esta capacidad son en su mayoría relacionados con el enfoque de la naturaleza y el control de los sistemas de actuación articular. En la actualidad, los dispositivos comerciales presentan articulaciones con actuadores rígidos que no permiten adaptarse a entornos impredecibles. Estos actuadores pueden consumir más energía y pueden no ser apropiados para la interacción hombremáquina. Actuadores adaptables están siendo diseñados e implementados debido a su capacidad para reducir al mínimo las fuerzas debido a choques, por su capacidad de interactuar de forma segura con el usuario, así como su capacidad para almacenar y liberar energía en elementos elásticos. Muchos estudios basados en simulaciones han evaluado los beneficios de incorporar articulaciones adaptables en los exoesqueletos. Movimientos espasmódicos y espasticidad son comunes entre los pacientes con deficiencias motoras; y debido a estos estudios actuadores adaptables pueden absorber eficientemente estas perturbaciones y mejorar el control de las articulaciones.

Este trabajo doctoral apunta a avanzar más allá del estado del arte en sistemas de actuación articular para exoesqueletos de marcha con los propósitos de: permitir la adaptabilidad de las articulaciones a distintas sintomatologías y mejoras en la eficiencia energética, así como brindar 
mayor adaptabilidad durante el caminado. Mediante el análisis de la biomecánica de la locomoción, se han identificado y analizado las características y requerimientos de las principales articulaciones involucradas en el ciclo de locomoción dinámica. Este trabajo doctoral presenta el diseño y desarrollo de dos novedosos actuadores adaptables que cumplen con los requisitos para la actuación de las articulaciones de exoesqueletos. La característica principal de los nuevos sistemas es que los elementos que permiten la adaptabilidad, simultáneamente permiten la medición del par ejercido por la articulación.

Concebido como actuadores adaptables controlados en fuerza, estos actuadores con rigidez ajustable y sensor incorporado, ARES y ARES-XL están destinados a ser implementados en las articulaciones del exoesqueleto pediátrico ATLAS. El dispositivo resultante es un exoesqueleto adaptable y controlado en fuerza, para niños con enfermedades neuromusculares que permite la explotación de la dinámica intrínseca durante el ciclo de locomoción. Las capacidades de ARES son presentadas y evaluadas, demostrando sus capacidades de medición de par del motor a diferentes niveles de rigidez.

La versatilidad de las articulaciones, como en el caso de la rodilla, puede ser emulada y aprovechada al incorporar elementos que puedan controlar el uso de la energía almacenada en las fases apropiadas de la marcha. ARES-XL permite la implementación de un mecanismo de bloqueo en combinación con su capacidad de cero rigidez y gran rango de deflexión. La evaluación del sistema demuestra cómo este diseño excede las principales capacidades de la realización original, a su vez el nuevo sistema proporciona una actuación versátil que podría conducir a su aplicación en múltiples articulaciones.

Durante este trabajo una evaluación del exoesqueleto-adaptable se realizó caminando bajo ciertas restricciones mecánicas. Comparando el comportamiento de las articulaciones a diferentes condiciones de rigidez, la adaptabilidad inherente de los actuadores presentados mostró adaptabilidad natural durante el ciclo de la marcha, y regiones de absorción de choque.

Se espera que el trabajo desarrollado en esta tesis doctoral continúe implementándose en aplicaciones de exoesqueletos y prótesis robóticas, a nivel de investigación y comercial. Varias publicaciones en revistas relevantes y conferencias internacionales han sido publicadas como consecuencia de la investigación llevada a cabo durante este trabajo de doctorado. Actualmente hay tres patentes producto de esta investigación, que están siendo explotados comercialmente por 
una PYME especializada en robótica para la salud. Los trabajos futuros se centrarán en la optimización del tamaño y peso de los sistemas de actuación adaptables, combinado con el desarrollo e implementación de estrategias de control adaptadas a los usuarios específicos y condiciones del entorno, con el objetivo de mejoras en la eficiencia energética y un andar más natural. 


\section{Table of Contents}

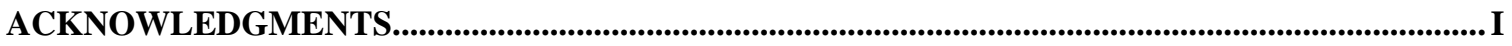

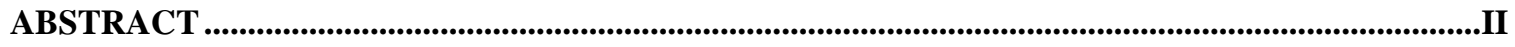

RESUMEN ......................................................................................................................................................... IV

LIST OF FIGURES .............................................................................................................................................. XI

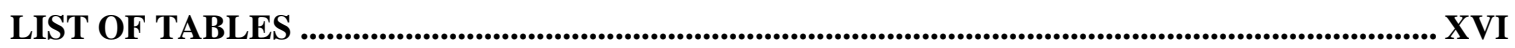

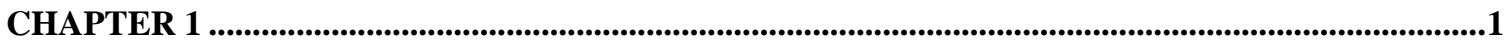

INTRODUCTION ...................................................................................................................................................1

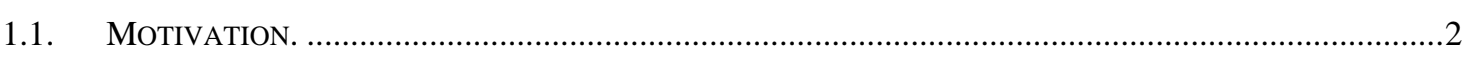

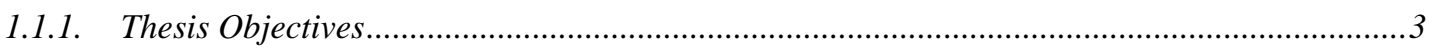

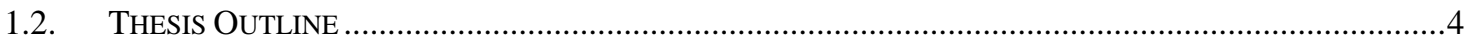

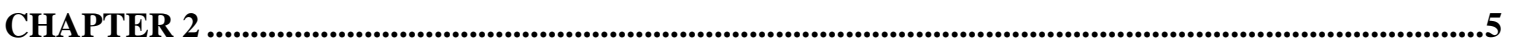

ATLAS PROJECT \& ADVANCES BEYOND THE SOA ON LOWER LIMB

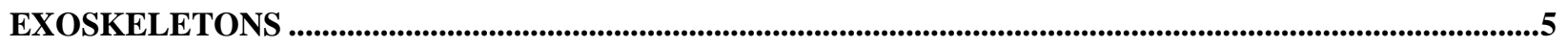

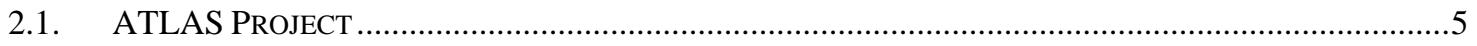

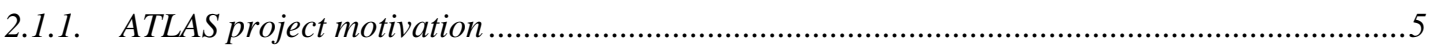

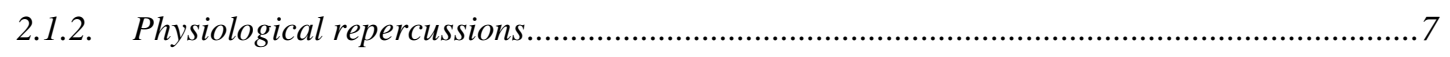

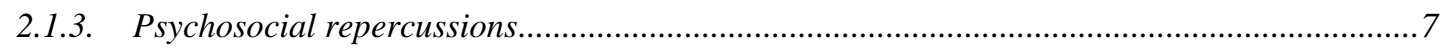

2.2. LOWER LIMB EXOSKELETONS- STATE OF THE ART ...............................................................

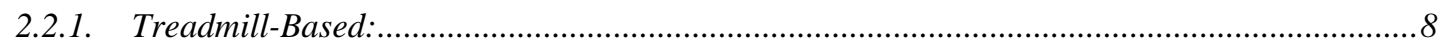

2.2.1.1. The Lokomat (Hocoma):............................................................................................................ 8

2.2.1.2. Lower-extremity Powered Exoskeleton (LOPES by the University of Twente):................................. 8

2.2.1.3. The Active Leg Exoskeleton (ALEX by the University of Delaware):.............................................. 8

2.2.1.4. Automated Locomotion Training using and Actuated Compliant Robotic Orthosis (ALTACRO by The Vrije Universiteit Brussel):

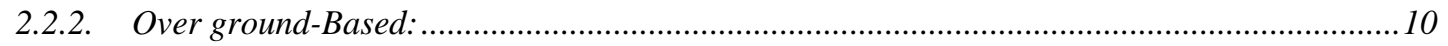

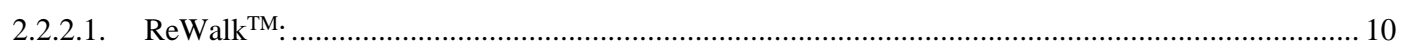

2.2.2.2. Rex:

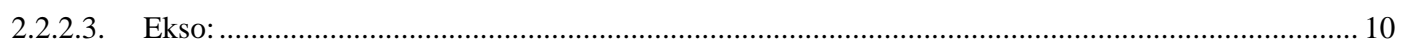

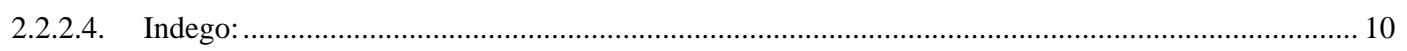

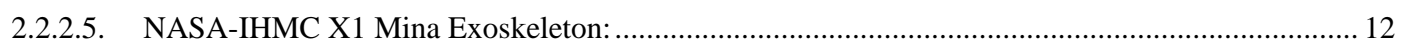




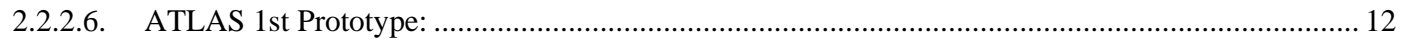

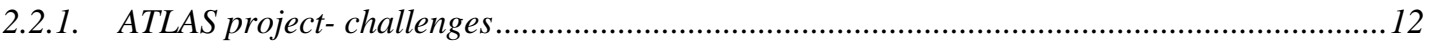

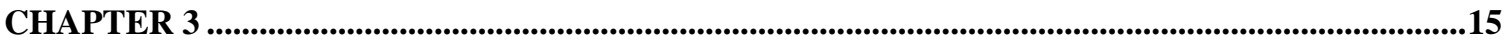

HUMAN GAIT - THE BIOMECHANICS OF LOCOMOTION....................................................15

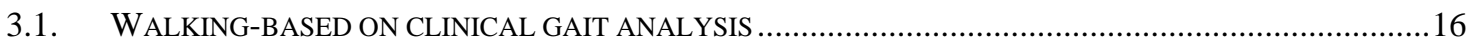

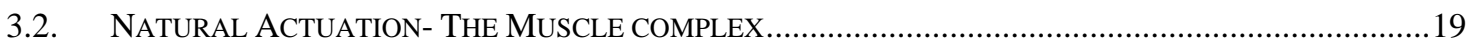

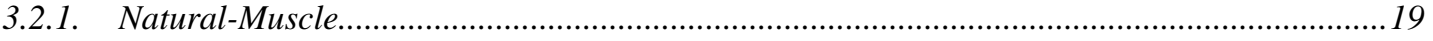

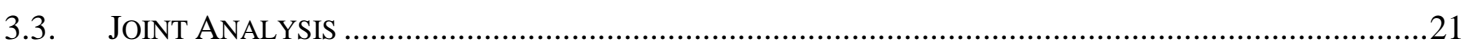

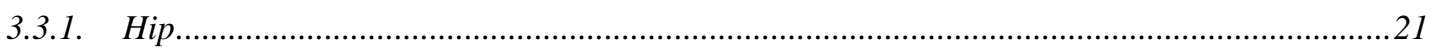

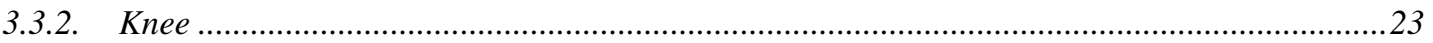

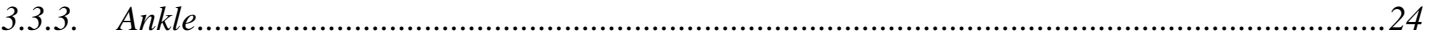

CHAPTER 4 ................................................................................................................................28

COMPLIANT ACTUATION- VARIABLE STIFFNESS ACTUATORS (VSA) ..........................28

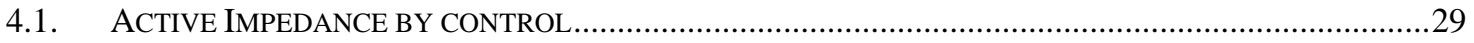

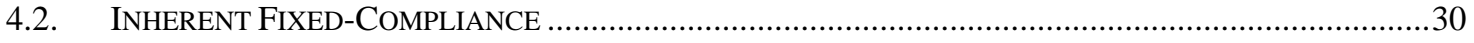

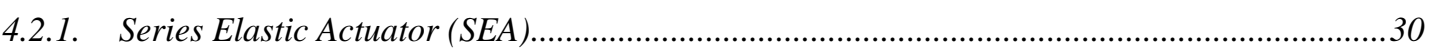

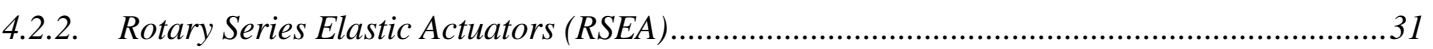

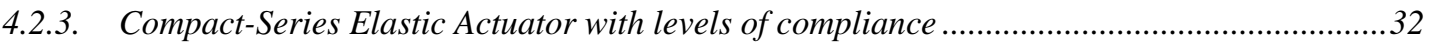

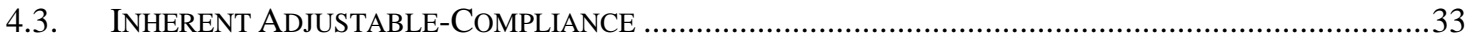

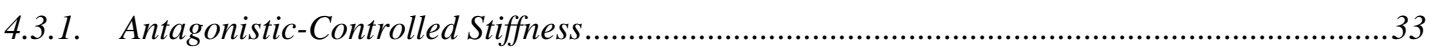

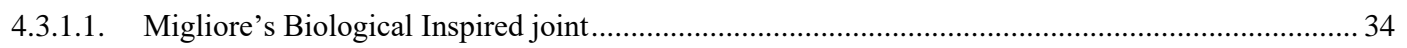

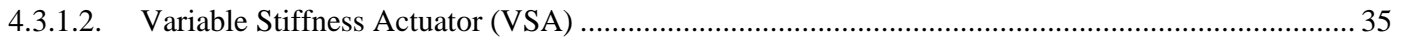

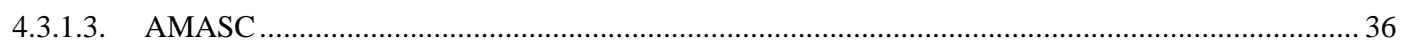

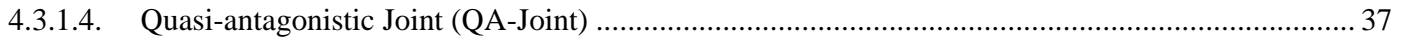

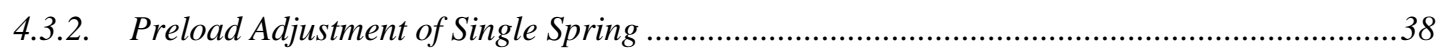

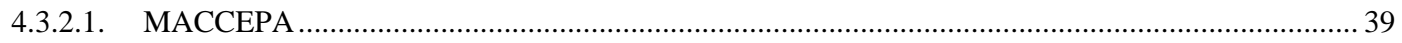

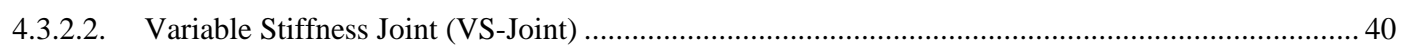

4.3.3. Changing Transmission between Load and Spring-Lever Length......................................41

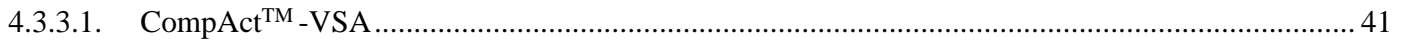

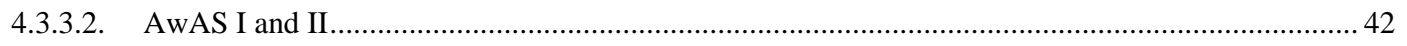

4.3.4. Controllable Variable Impedance Actuators (VIA) ....................................................43

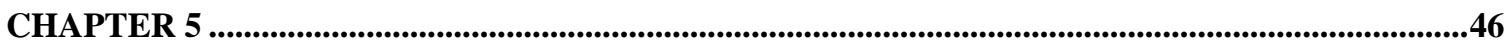

\section{ACTUATOR OF ADJUSTABLE RIGIDITY WITH EMBEDDED SENSOR-}

\section{ARES $\quad 46$}

5.1. COMPLIANT JOINT REQUIREMENTS... 


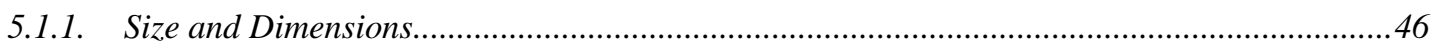

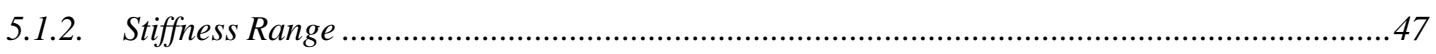

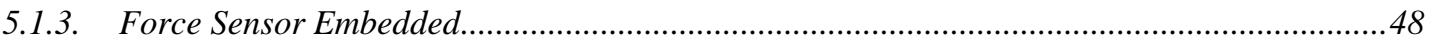

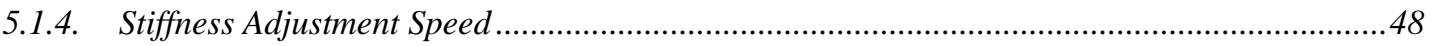

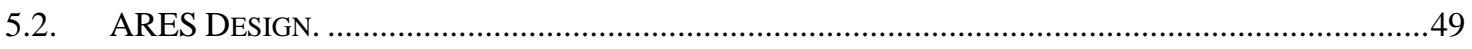

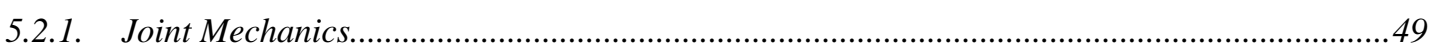

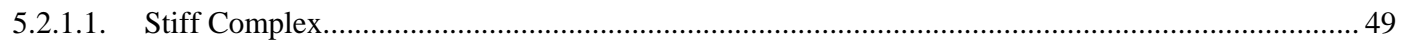

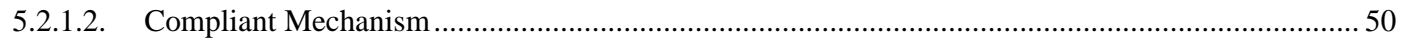

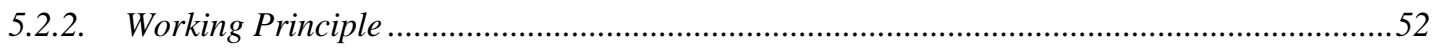

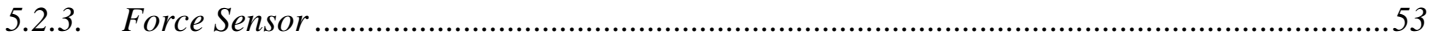

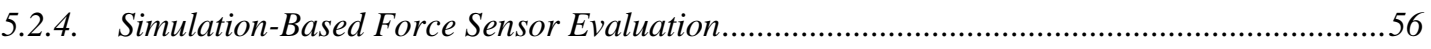

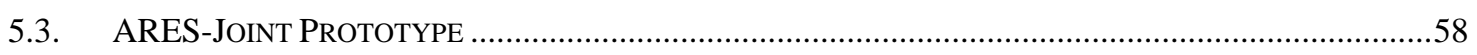

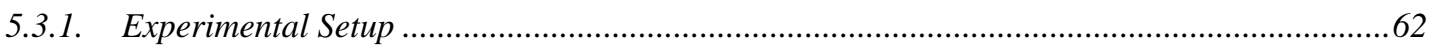

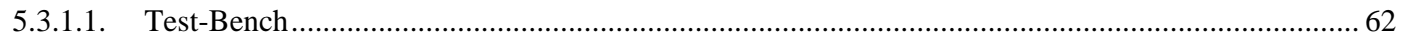

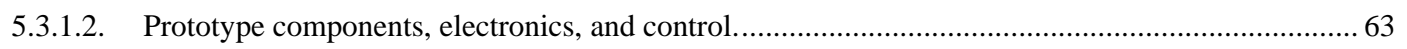

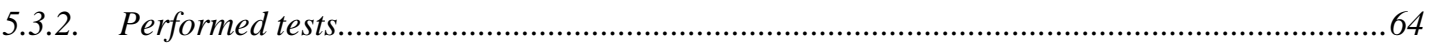

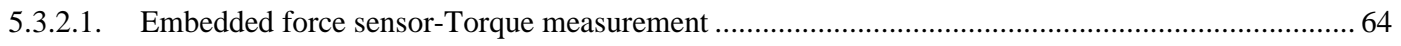

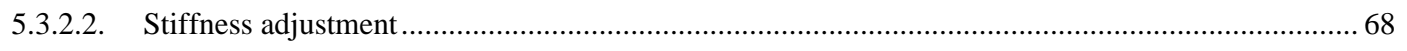

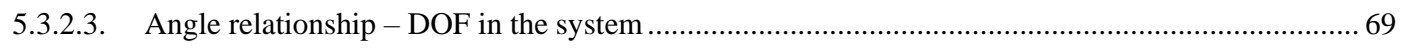

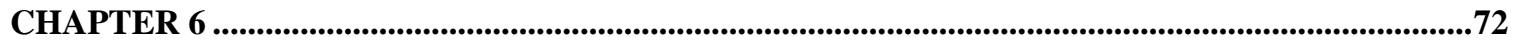

\section{ACTUATOR OF ADJUSTABLE RIGIDITY WITH EMBEDDED SENSOR}

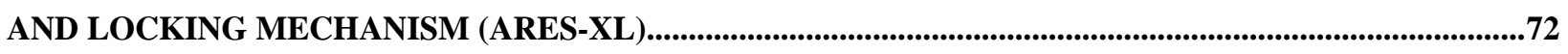

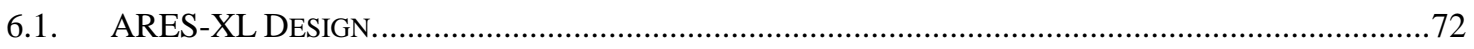

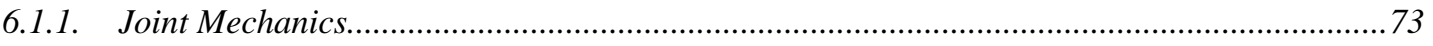

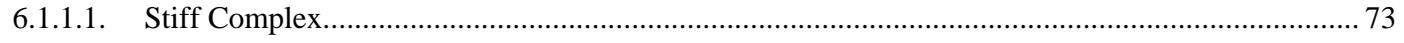

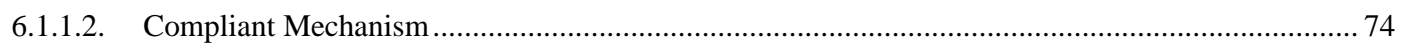

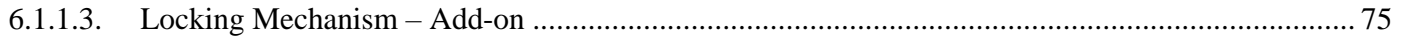

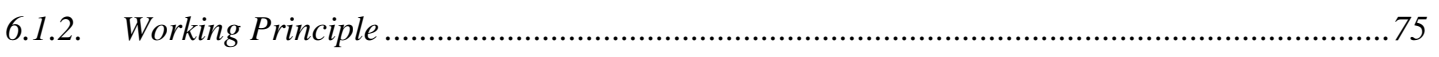

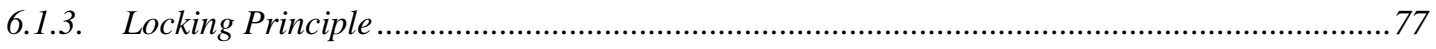

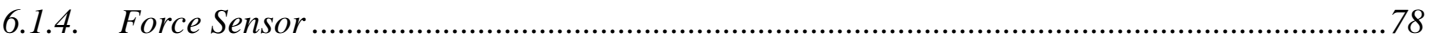

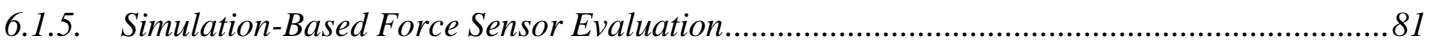

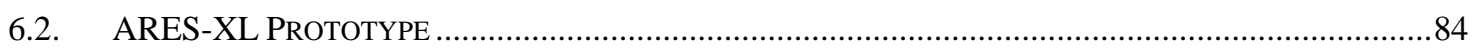

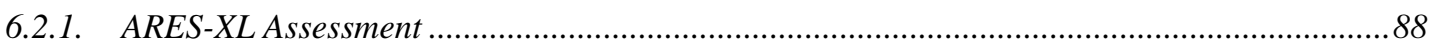

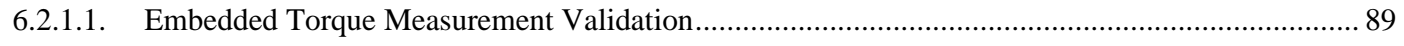

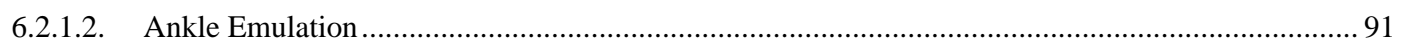

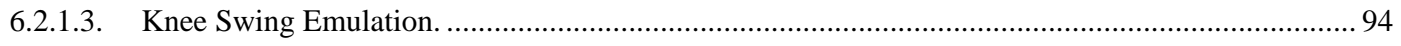

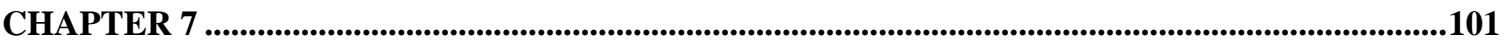




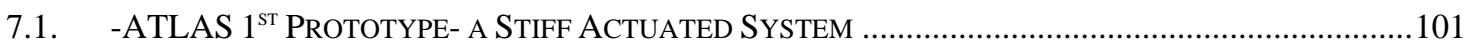

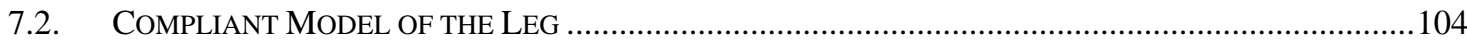

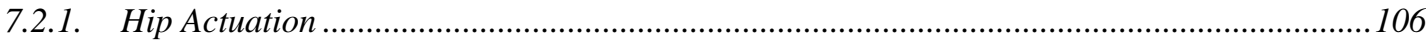

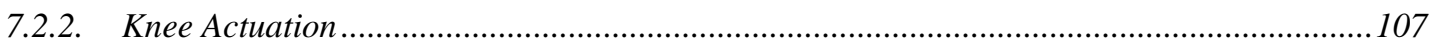

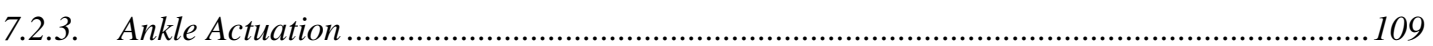

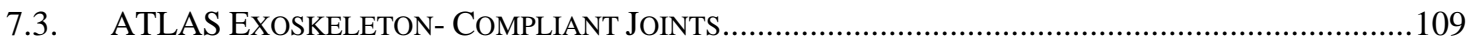

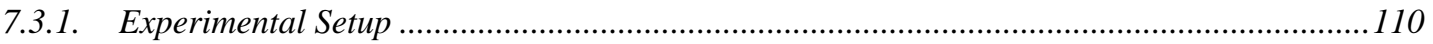

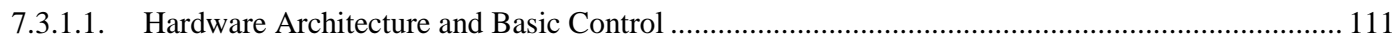

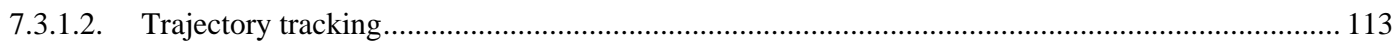

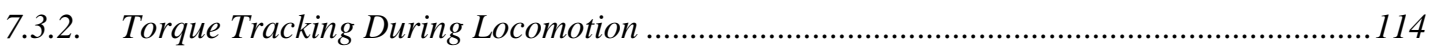

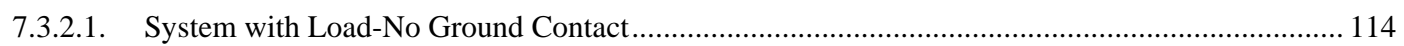

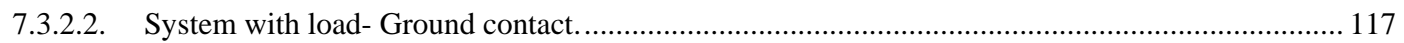

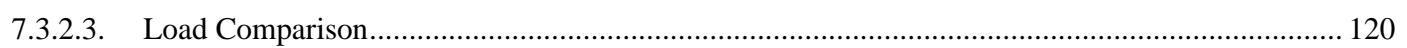

7.3.3. Gait Control Strategy Based on State Machine ……….................................................... 123

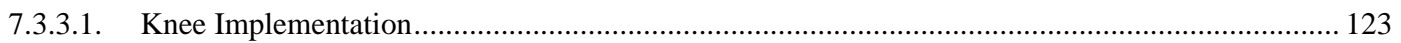

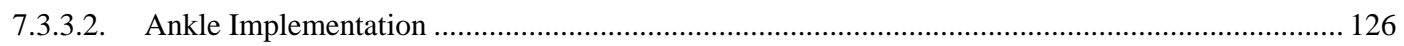

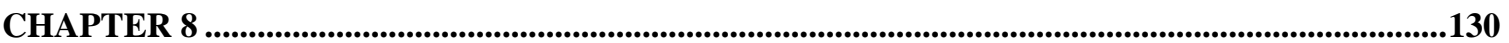

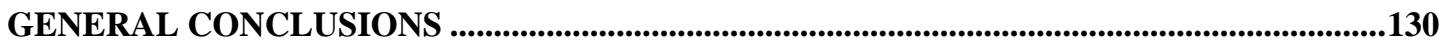

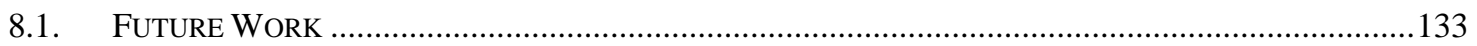

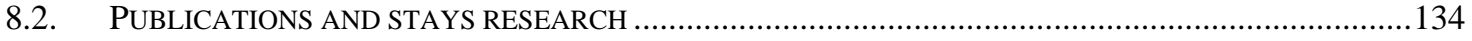

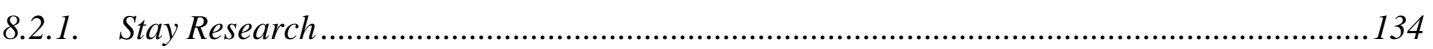

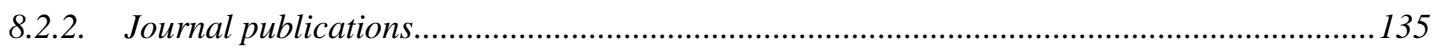

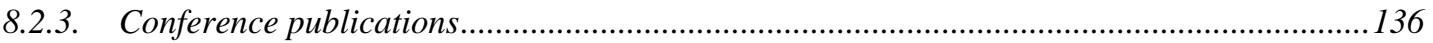

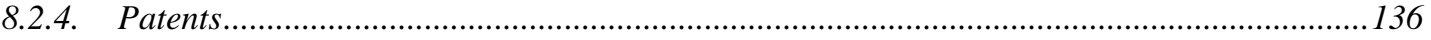

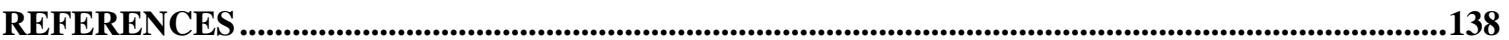




\section{List of Figures}

Figure 2-1. Distribution of cases of mobility impairment.

Figure 2-2. Treadmill-Based Exoskeletons. (a) Lokomat, (b) LOPES, (c) ALEX,

(d) ALTACRO.

Figure 2-3. Over Ground-Based Exoskeletons. (a) ReWalk ${ }^{\mathrm{TM}}$,(b) REX Bionics, (c)

Ekso Bionics, (d) Indego, (e) X1-Mina Exoskeleton, (f) ATLAS Lower Limb

Exoskeleton. 11

Figure 2-4. Challenges in Rehabilitation Exoskeletons' Design. ............................................. 13

Figure 3-1. Planes of Section of the Human Body ..................................................................... 15

Figure 3-2. Data from Clinical Gait Analysis (CGA) and Phases of the Locomotion

Cycle- Right Leg. 17

Figure 3-3. Clinical Gait Data. Angular Position, Moment, and Power of the

Sagittal Joints [54]. Walking Cycle Moderate-Speed............................................................. 18

Figure 3-4. Multifunction of the Natural Muscle................................................................... 19

Figure 3-5. Classic Hill-Muscle Model ..................................................................................... 20

Figure 3-6. (a) Hip-Impedance and (b) Hip-Leg Model to Fulfill Hip Requirements................. 22

Figure 3-7. Knee Analysis. (a) Sub-Phases at the Knee During Gait. (b) Knee-

Impedance Evolution During Gait. (c-d-e) Knee-Leg Model to Fulfill knee

Requirements.

Figure 3-8. (a) Ankle-Impedance Evolution During Gait. (b-c-d) Ankle-Leg Model

to Fulfill Ankle Requirements.

Figure 4-1 . a) Schematic Diagram of Series Elastic Actuators [45] b) Series Elastic

Actuator. 31

Figure 4-2. Rotary Series Elastic Actuator Schematics.................................................... 32

Figure 4-3. Powered Ankle with Compact Series Elastic Actuator........................................ 33

Figure 4-4. Migliore's Biological Inspired Joint and Quadratic Spring Device......................... 34

Figure 4-5. VSA-I. a) VSA Prototype b) Tensioning Mechanism. ........................................ 35 
Figure 4-6. 4 Linkage Bar Mechanism of the VSA-II. 36

Figure 4-7. 4 AMASC-a) Schematic Diagram of Pulleys Mechanism and b) $1^{\text {st }}$ Leg

Prototype.... 37

Figure 4-8. Quasi-Antagonistic Joint. a) Transmission Variable Design. b) QA-

Joint and Principle of Operation. 38

Figure 4-9. a) MACCEPA Basic Configuration. b) MACCEPA Prototype. c)

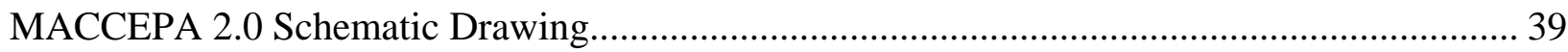

Figure 4-10. a) Mechanism of the VS-Joint. b) Working Principle of the VS-Joint. .................. 40

Figure 4-11. CompAct-VSA Mechanism.

Figure 4-12. a) AwAS I Mechanical Principle and b) AwAS II Mechanical

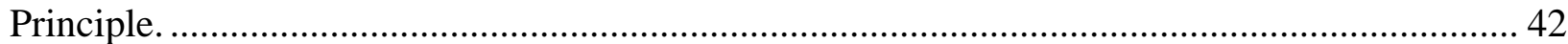

Figure 4-13a) AwAS b) AwAS II CAD prototypes............................................................ 43

Figure 5-1. ARES Joint Mechanics. a) CAD Drawing of ARES, b) CAD of Stiff

Complex, and c) CAD of Compliant Mechanism............................................................... 50

Figure 5-2. ARES Components Coupling ............................................................................. 51

Figure 5-3. ARES Working Principle. a) Less Stiffness Configuration, b) High

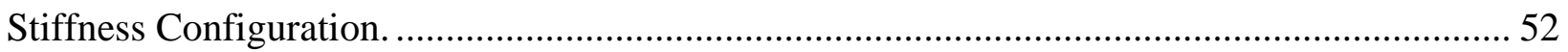

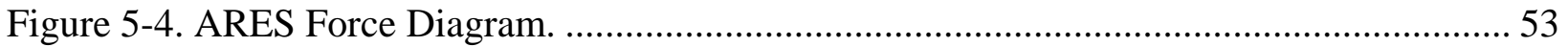

Figure 5-5. ARES Range of Stiffness. a) Torque Range for Different Positions of

Lo b) Stiffness Range for Different Positions of Lo........................................................... 55

Figure 5-6. Open-Loop Tracking Simulation with Saturated Measurement ............................ 56

Figure 5-7. Low Torques Tracking Simulation in Open-Loop............................................. 57

Figure 5-8. Open-Loop Tracking Simulation with Ramps and Steps Inputs............................. 58

Figure 5-9. ARES Joint- CAD Model with Main Dimension. ................................................. 60

Figure 5-10. ARES Prototype.a) ARES Prototype-Front View. b) ARES Complian

Mechanism' Prototype-Front View. c) Main Actuation -M1 ............................................ 61

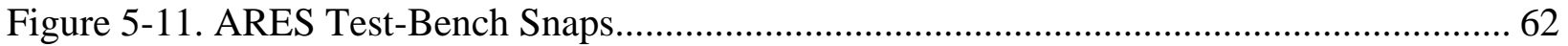

Figure 5-12. Simplified Schematic of the Experimental Control Setup.................................. 64

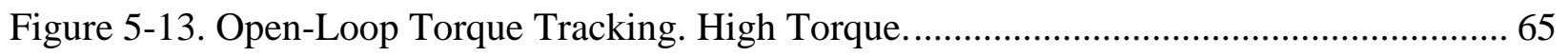

Figure 5-14. Open-Loop Torque Tracking- Low Torque. ..................................................... 66

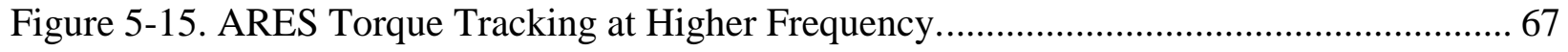




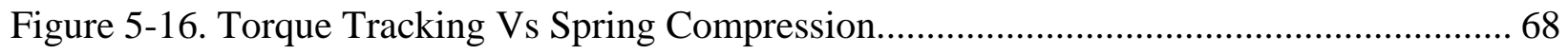

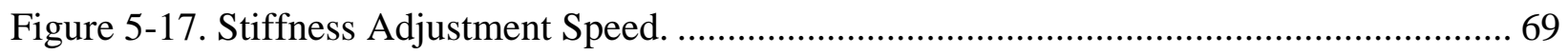

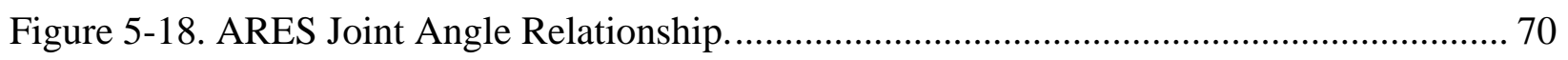

Figure 6-1. ARES-XL Joint Mechanics. a) CAD Drawing of ARES-XL, b) CAD of

Compliant Mechanism, c) Locking Mechanism -Add on, and c) CAD of Stiff

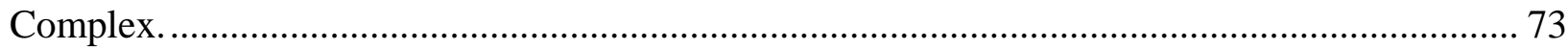

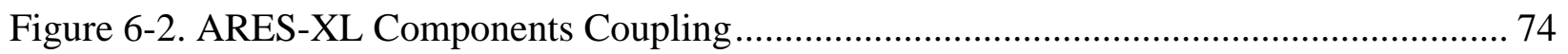

Figure 6-3. ARES-XL Working Principle. a) ARES-XL High Stiffness b) ARES-

XL Minimum Stiffness. 76

Figure 6-4. ARES-XL Locking Mechanism. a) Tooth and Pawl Engaged while

Joint Loaded in Opposite Direction. b) Slider Change Position, Pulling the Pawl to

Disengage. c) Pawl Disengaged- the Joint Moved Freely Due to Load and Springs................. 77

Figure 6-5. ARES-XL, Diagram for Torque Calculations................................................ 78

Figure 6-6. ARES-XL Range of Stiffness. a) Torque Range for Different Positions

of Lo b) Stiffness Range for Different Positions of Lo. ...................................................... 81

Figure 6-7. Open-Loop Torque Tracking Simulation with Different Sine Torques................... 82

Figure 6-8. Ramps and Steps Torque- Simulation in ARES-XL ......................................... 83

Figure 6-9. ARES-XL Joint- CAD Model with Main Dimension............................................ 85

Figure 6-10. ARES-XL Prototype.a) ARES-XL Prototype-Front View. Pawl -L1

not Being Pulled. b) ARES-XL Prototype-Front View. Pawl -L1 Being Pulled and

Stiffness Near Zero. c) View of the Locking System-Ratched with Pawl

Disengaged. d) View of the Locking System-Ratched with Pawl and Locking

Engaged. e) ARES-XL Prototype -Custom View. Coupling Elements Highlighed.................... 86

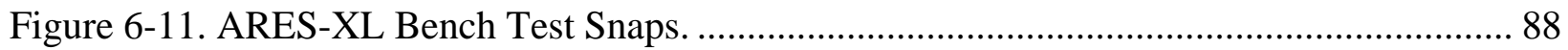

Figure 6-12. Torque Tracking in ARES-XL at 2 Levels of Stiffness and $1.6 \mathrm{Kg}$

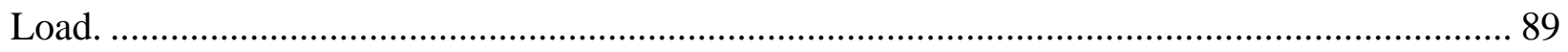

Figure 6-13. Torque Tracking in ARES-XL at 2 Levels of Stiffness and $3.6 \mathrm{Kg}$

Load. 90

Figure 6-14. State-Machine at the Joint to Emulate the Ankle............................................ 91

Figure 6-15. Ankle Emulation. a) ARES-XL Position Response. b) Stiffness Levels

-State Machine 92 
Figure 6-16. Ankle Emulation. a) ARES-XL Joint Velocity. b) Current

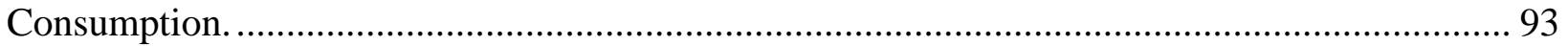

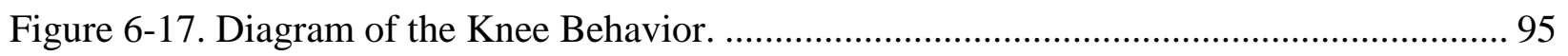

Figure 6-18. Schematics of the State-Machine Applied to Emulate the Knee Swing................ 96

Figure 6-19. Knee-Swing Emulation. a) ARES-XL Joint Position and Trajectory. b)

ARES-XL Velocity. c) ARES-XL Deflection and Stiffness Levels State Machine.................... 97

Figure 6-20. ARES-XL Locking and Unlocking Sequence. Locking: Not engaged

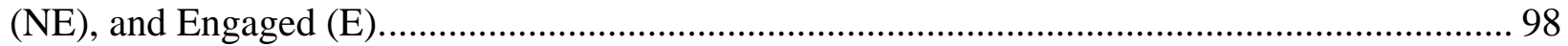

Figure 7-1. Exoskeleton Prototype with Stiff Actuation ................................................... 102

Figure 7-2. Walker Frame for Balance Control and Center of Mass Vertical

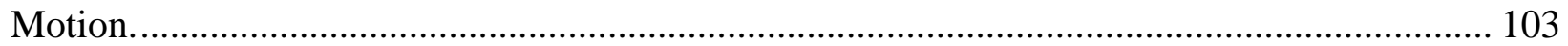

Figure 7-3. Leg Model- Levels of Stiffness ................................................................. 105

Figure 7-4. ARES vs ARES-XL Stiffness Ranges for System Selection ............................... 106

Figure 7-5. ARES-Simplification, a SEA Rotary Actuator for the Hip.................................. 107

Figure 7-6. ATLAS Exoskeleton- Prototype with Compliant Joints....................................... 110

Figure 7-7. Exoskeleton and Walker Frame Coupled. CoM Vertical Displacement

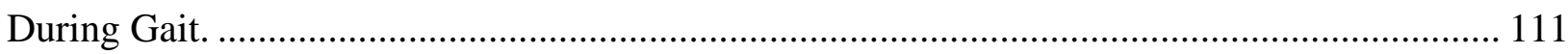

Figure 7-8. Simplified-Control Diagram of the ATLAS Compliant Exoskeleton..................... 112

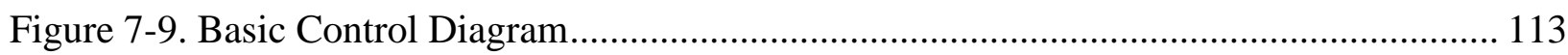

Figure 7-10. Commanded Joints Trajectories. Highlighted Area Where Leading

Leg Waits for the Leg Swing of the Other Leg. ............................................................ 114

Figure 7-11. Torque Tracking Under Different Stiffness for Knee and Ankle Joint-

Hip in the Air With Load.......................................................................................... 115

Figure 7-12. Torque Tracking for Different Stiffness- Knee in the Air With Load................. 116

Figure 7-13. Torque Tracking for Different Stiffness- Knee in the Air With Load.................. 117

Figure 7-14. Torque Tracking for Different Stiffness at Knee and Ankle- Hip in the

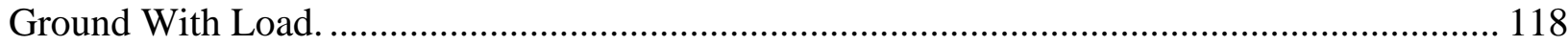

Figure 7-15. Torque Tracking for Different Stiffness- Knee in the Ground With

Load.

Figure 7-16. Torque Tracking for Different Stiffness- Ankle in the Ground With

Load.. 119 
Figure 7-17. Torque Tracking at the Hip under Different Conditions of Load....................... 120

Figure 7-18. Torque Tracking at the Knee Under Different Conditions of Load..................... 121

Figure 7-19. Torque Tracking at the Ankle Under Different Conditions of Load. ................... 122

Figure 7-20. State Machine Commanded at the Knee. a) SM- for Lock

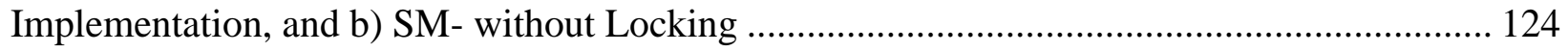

Figure 7-21. Knee Results with State Machines Implemented.......................................... 125

Figure 7-22. Knee Results with State Machines Implemented- Velocity and

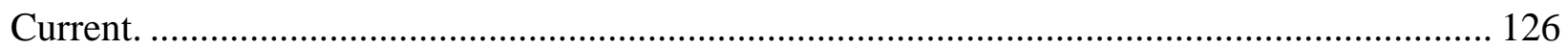

Figure 7-23. State Machine Commanded at the Ankle (Ankle-SM). ................................... 127

Figure 7-24. Position and Deflection when Implementing Ankle-SM................................ 128

Figure 8-1. ATLAS- Compliant Prototype and CAD design. ............................................. 134 


\section{List of Tables}

Table 2-1. World and Europe group's distribution of affected individuals..................................... 6

Table 2-2. Basic Comparison of Over-Ground Exoskeletons. ................................................... 14

Table 3-1. Joint characteristics during gait, based on the MTU actuation. .................................. 26

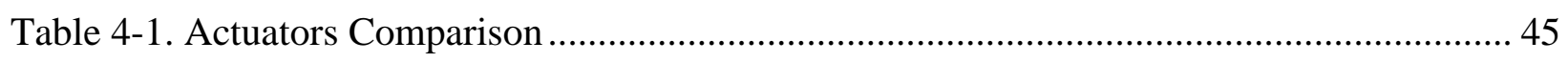

Table 5-1. Different Stiffness Ranges at the Knee Joint............................................................ 48

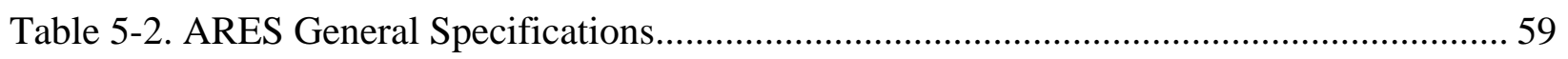

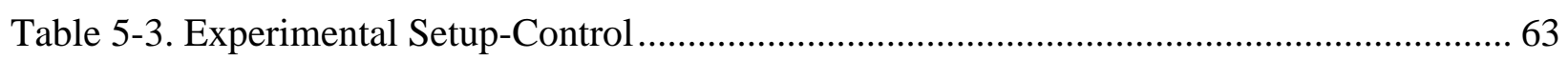

Table 5-4. Actuators Comparison including ARES.................................................................. 71

Table 6-1. ARES-XL and ARES Mechanical Properties ............................................................ 84

Table 6-2. Actuators Comparison Including ARES and ARES-XL........................................... 100

Table 7-1. Summary of the Torque Tracking experiment's Results............................................ 122

Table 7-2. Over-Ground gait Exoskeletons and ATLAS-C...................................................... 129 


\section{Chapter 1}

\section{Introduction}

There are several causes that affect the lower limb mobility worldwide. Current estimations inform spinal cord injury (SCI) affects 3 million people around the world. About 265.000 spinal cord injured individuals, just in the United States [55]; over 30,000 affected and diagnosed approximately 1,400 new spinal cord injuries per year in Spain. Moreover, neuromuscular diseases (NMD) are a group of more than 150 inherited or acquired neurological diseases affecting the muscles and nervous system. It affects one in 10,000 children worldwide. In overall terms, there are more than 4 million people affected by neuromuscular diseases in the world and more than 50,000 affected in Spain [54].

The spinal cord injury can derives in paraplegia or quadriplegia resulting in the loss of mobility, and there is no range of ages of affected individuals, as is a consequence of an injury. On the other hand, Spinal Muscular Atrophy (SMA) one of the most common NMD begins in childhood and is clinically manifested by significant hypotonia and muscle weakness, translated in the loss of walking ability among other consequences. Intensive-walking training appears to be vital to the activitytriggered learning process of the sensorimotor system. For people who will probably never walk again, assisted gait training diminishes the negative effects resulting from being bound to a wheelchair.

Recently, in an attempt to improve recovery and functionality, there has been a considerable increase in the implementation of robotic devices, such as exoskeletons and active orthoses that help people with walking disabilities to regain strength in their legs and joints so they can stand or even walk again. For the purpose of this work, an exoskeleton is defined as a mechanical device, essentially anthropomorphic in nature, worn by an operator and that fits closely and operates in parallel with the human legs. The effort to restore mobility to the legs of these patients has encouraged the development of many devices, many of them already available commercially. These devices use electric motors that require a large gear reduction to achieve the desired torque for exoskeleton joints while maintaining a small size. However, when physical interaction with the world is required while maintaining interaction with the user, particularly interaction that involves an impact or kinetic energy transfer 
[25], the traditional approach of using stiff actuators from classical robotic applications to hold precise positions [22] is not ideal.

\subsection{Motivation.}

There are several limitations in the current technology and applications of robotic exoskeletons. They reproduce a programmed gait pattern by actively controlling the motion of only two joints per leg, or significantly changing the natural gait. As the number of degrees of freedom of the human leg is significantly larger than two (the simplest mechanical model of the human leg identifies 7 degrees of freedom [12]), this actuation results in a non-physiological gait which is not indicated for neuromuscular rehabilitation. Also, because of featuring a limited number of controlled degrees of freedom, the device cannot provide balance while maintaining reduced size and weight, so the user needs to make use of crutches or walkers to maintain balance during standing and walking. The use of such devices requires physical strength in arms and hands, as the crutches must support the extra weight of the robotic device during some phases of the gait. NMD children present acute weakness in arms and hands so they are unable to make use of these stabilizing aids. Also, thoracic control and weight-bearing capacity are required for the use of current commercial exoskeletons. Tetraplegics, and NMD children do not have these capabilities. Besides the previous reasons, also these devices are not suitable for children, as the minimum height of the user accepted is $1,54 \mathrm{~m}$. However, the main reason for these commercial devices for not being indicated to the treatment of children with NMDs is their inability to safely interact with the user symptomatology.

Many studies suggest that one reason why biological systems can successfully interact with the environment is that they have the ability to modulate joint impedance [15], understanding mechanical impedance as the additional force created at the output by load motion. The implementation of an impedance control approach requires the actuator to be a high-precision force source [58]. A complete active control of the joint impedance in active orthosis applications suffers from some drawbacks: Most implementations of impedance control techniques using force-feedback, whit traditional stiffness-joints require a significant level of computation and requires a constant energy drain, as the motor must apply a constant force despite the fact that no work is being performed [22]. Commercial devices, for gait assistance and rehabilitation, nowadays present joints actuated with stiff actuators, which result in devices that cannot adapt to unpredictable environments, consume much energy and are not appropriate for human-machine interaction.

To develop powered devices that could assist the patient for long periods, adapt to their necessities, the incorporation of compliant joints to resemble the biological counterpart and achieve a 
safe human-robot interaction is nowadays a necessity. This doctoral work has been developed under the ATLAS project DPI2010-18702 (Performance and control of power amplification devices and mobility in humans and robots), this project aimed to establish the research in the design of new actuators and mechanical structures for the new generation of service robots, focused on its implementation for children with SMA, and SCI.

\subsubsection{Thesis Objectives}

This $\mathrm{PhD}$ Thesis intents to design and develop new actuators with high power-to-weight ratio with the capability of controlling its mechanical impedance if required. Some patients with neurological disorders often suffer from severe spasms. If rigid actuators are used, unwanted movements, like those of spasmodic type, can cause high stresses in the mechanical system that could cause damage to the patient's leg. Adding compliance to the actuators can allow absorbing significant position errors, thus avoiding damage to the system itself and of course, ensuring patient safety. Since the use of elastic elements can store energy during the foot support, this stored energy can be later used for power generation.

The proposed actuator integrates in its mechanism a force sensor that allows force feedback at the joint, taking advantage of the elastic elements that simultaneously regulate the joint impedance. Mass and inertia of the actuator are minimized by the compact design and the simultaneous use of each component for more than one utility (i.e. sensing and acting).

In order to accomplish the goals of this doctoral work, some main objective are traced.

The biomechanics of normal walking provides the basis for the design of new actuated artificial limbs. Studying, and identifying the main requirements of the sagittal joints, during the locomotion cycle will allow to select the elements to incorporate into this thesis work. The design, and manufacture of the desire variable impedance actuators with embedded sensors, with application to the joints of ATLAS exoskeleton. This work will validate the embedded force sensor, and compliant systems by testing the systems prototypes.

The developed actuators will be implemented into an exoskeleton structure. The designed and manufactured robotic device with 3DOF per leg, provided with compliant joints will finally be evaluated. The behavior of the compliant exoskeleton during locomotion will be assessed. Focusing on exploiting the proposed actuators properties and locomotion dynamics, different experimental setups with, and without special control strategies will be tested. 


\subsection{Thesis Outline}

During this doctoral thesis, two compliant systems were built. The Adjustable Stiffness with Embedded Sensor actuators ARES, and ARES-XL were developed as versatile devices with intrinsic properties that could be exploited by means of a proper control strategy during the locomotion cycle. Adaptability, force control, and energy efficiency are some of the main properties of the developed devices.

The dissertation is divided in 7 chapters besides this introductory chapter. An introduction to the ATLAS project and a review of current exoskeleton technologies is given in Chapter 2. Following an important principle in science, where one must understand the normal behavior of a phenomenon before attempting to study the abnormal or pathological; Chapter 3 presents an analysis of the biomechanics of locomotion. The study of the natural actuation, along with the understanding of the main requirements of the lower limb' joints contribute with the design and development of a suitable system for providing actuation to robotic exoskeleton.

A review of the current state of the art on compliant actuation technologies is given in Chapter 4 , based on this chapter the limitations of the technology are identified and a novel design is introduced in Chapter 5. The ARES actuator' design and functionalities are presented in this chapter, along with several simulations and evaluation of the actuator prototype.

ARES-XL, an improved version of the original realization ARES, is presented in Chapter 6. The deflection, and stiffness capabilities of this system exceed the achieved with the previous prototype. The evaluation of the system is presented in this chapter, in combination with some control strategies intended to exploit the actuator capabilities during the walking cycle.

Chapter 7 introduces the assessment of the first compliant exoskeleton for children, coupled with a special walker frame to provide balance while allowing a semi-natural gait. The behavior of the exoskeleton is discussed along the chapter, and online adaptations of the actuator's stiffness are commanded, combining control, and exoskeleton reactions to exploit the kinetic, potential, and dynamic properties of walking and the compliance. Finally, general conclusion and future work recommendation of this doctoral thesis are given in Chapter 8.

This doctoral work was supported by the Spanish Ministry of Economy and Competitiveness performed under the frame of the ATLAS project developed at the Centre for Automation and Robotics (CAR), joint center by the Spanish National Research Council and the Polytechnic University of Madrid. 


\section{Chapter 2}

\section{ATLAS Project \& Advances beyond the SoA on Lower Limb Exoskeletons}

Research in robotic exoskeletons and active orthosis began in the early 1960s [50, 67]. However, it has been in the last few years that a significant increase in the development of these devices can be seen. Advances in robotic technologies, actuators, sensors, new materials, control algorithms, and miniaturization of computers have led to this growth [11]. The effort to help people with walking disabilities to regain strength in their legs and joints so they can stand or even walk again, has encouraged the implementation of these robotic systems for assistance and rehabilitation focusing on providing missing movements and sensing to the user in a safe environment. Robotic rehabilitation not only provides consistent and efficient therapy without exhaustion, it also has the potential to enhance the therapy beyond the abilities of the practitioner [34].

\subsection{ATLAS Project}

The ATLAS project ( New Actuation and Control Technologies for Empowering Humans and Robots DPI2010-1872), a research project developed at the Centre for Automation and Robotics (CSIC-UPM) had the main purpouse to progress beyond the state of the art in these scientific and technical issues that currently limit the development and real-world application of portable exoskeletons and active orthoses.

To demostrate the project results and as part of the development of this work, a lower limb exoskeleton that can be considered as an THKAFO (Trunk-Hip-Knee-Ankle-Foot Orthosis) intended to help with walking at a moderate speed $(<0.8 \mathrm{~m} / \mathrm{s})$ to children with walking disabilities, has been designed, developed and tested. This technology has been licensed to Marsi Bionics, a SME specialized on robotics for healthcare [36].

\subsubsection{ATLAS project motivation}

Approximately $1 \%$ of the world's population depends on wheelchairs to move. This means that about 60 million people in the world depend on a wheelchair. With the aging of the Western population 
this number increases beyond measure. While wheelchairs have improved in quality in recent decades, the main limitation on autonomy, independence, health and personal integrity continues to exist with the use of the wheelchair [71].

Current estimations inform about 265.000 spinal cord injured individuals, just in the United States [55]. The spinal cord injury derives in paraplegia or quadriplegia resulting in the loss of mobility. Spinal cord injury affects 3 million people worldwide; in Spain there are over 30,000 affected and diagnosed approximately 1,400 new spinal cord injuries per year (incidence rate: 30 per million inhabitants), in the Community of Madrid about 1102.

Neuromuscular diseases are a group of more than 150 inherited or acquired neurological diseases affecting the muscles and nervous system. It affects one in 10,000 children worldwide. In overall terms, there are more than 4 million people affected by neuromuscular diseases in the world and more than 50,000 affected in Spain [54].

Totaling all causes, the total affected by lower limb paralysis in the world amounts to nearly 24 million people. To this total must be added the group of elderlies in the world that depend on a wheelchair, which are about 40 million. Table 2-1, and Figure 2-1 present the distribution of affected cases in the world and Europe, where in Europe about 15\% are children affected mainly by Cerebral Palsy and Neuromuscular diseases.

Table 2-1. World and Europe group's distribution of affected individuals.

\begin{tabular}{lcc}
\hline & People in the World & People in Europe \\
\hline Elderly & $40,000,000$ & $15,000,000$ \\
Cerebral Palsy [49] & $14,000,000$ & $1,879,700$ \\
Neuromuscular diseases [17] & $4,000,000$ & 783,200 \\
Spina bifida [33] & $3,000,000$ & 300,400 \\
Spinal cord injury [55] & $3,000,000$ & 450,000
\end{tabular}
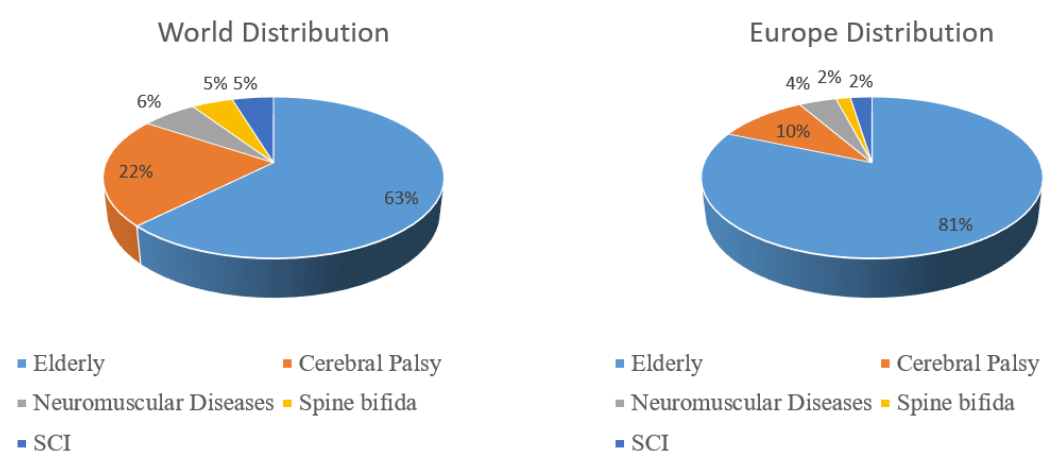

Figure 2-1. Distribution of cases of mobility impairment. 


\subsubsection{Physiological repercussions}

The lack of mobility in the lower limbs and the continued sitting position causes significant physiological disorders. Disorders in sexuality and fertility, loss of bladder control and feces are very common. But also because of reduced mobility and loss of the ability to walk, paraplegia can cause numerous medical complications. Some of the most common complications include pressure ulcer, spasticity, tissue necrosis, thrombosis and pneumonia. Physical therapy and some technological devices, such as the stander or standing frame can help prevent these complications [71].

Pain is a common problem after a traumatic injury to the spinal cord. Their frequency is from $42 \%$ to $69 \%$, identified as a significant cause of patient activity limitations and contributing to a decrease in quality of life. Some studies have suggested that chronic pain in these patients may be more associated with psychosocial factors than to physiological factors [35].

\subsubsection{Psychosocial repercussions}

The loss of mobility in the lower limbs creates dependency, affects the self-confidence and self-esteem, and reduces employment opportunities and social interaction and economic stability. Psychosocial conditions derived from paralysis may include feelings of frustration, embarrassment, humiliation, emotional distress, depression and long-term problems related to loss of employment, partner and wellness, and suicidal ideation, which often occurs in people with paraplegia [35].

Concern about the opportunities and living conditions of people in disability status, has motivated the development of policies and programs aimed at understanding this phenomenon from the perspective of people with disabilities and the environment. It seems that if you restore the function of the legs would solve many of the physiological and psychosocial loss arising from lower limb mobility. Robotic technology can help, if not regaining locomotor functions, yes providing mobility through an external orthopedic device that engages affected joints and execute the movement of his/her legs through servo-controlled motors, allowing patient to walk on his/her own, without help of others, providing independence, regaining lost physiological functions, including chronic pain relief to the extent that is caused by psychological problems arising from the lack of mobility. Such a device is called lower limb exoskeleton.

\subsection{Lower Limb Exoskeletons- State of the Art}

Based on the exoskeleton classification given in [7], the following review will focus in lower limb exoskeletons for medical applications, intended for rehabilitation and assistance, while robotic exoskeletons to augment the performance of the able body person are out of the scope of this work 
$[12,34]$. Several works agree in a subdivision inside exoskeletons for medical applications based on the training/working environment [7, 12, 28, 34].

\subsubsection{Treadmill-Based:}

Many robotic systems have been developed with the aim to automate rehabilitation techniques. A combination of an exoskeleton that can support totally or partially the weight of the patient in combination with a treadmill, in order to reproduce the natural gait and improve functional mobility. Some of the most relevant devices under this category are presented below.

\subsubsection{The Lokomat (Hocoma):}

A mechatronic body weight support (BWS) system [18, 34] developed to provide precise body weight unloading for patients with neurological or other impairments during treadmill training. This commercial gait therapy device provides and active BWS and a robotic exoskeleton support hip and knee movements in the sagittal plane. Trainings with the Lokomat system is traditional performed with a fixed gait pattern as the robot followed the predefined joint angle trajectories. Gait pattern algorithms were implemented in Lokomat to incorporate the assist as needed functionality. However, it is still based of fixed gait patterns and limited to sagittal motion.

2.2.1.2. Lower-extremity Powered Exoskeleton (LOPES by the University of Twente):

A compliant rehabilitation robot with $9 \mathrm{DOF}$, that besides the capabilities of the Lokomat, allows balance training [63]. It presents two parts, a 2-D pelvic control system and an exoskeleton with 2 DOF at the hip and 1 at the knee per leg. Bowden cables are used to realize a flexible transmission, so that the motors are detached from the exoskeleton, reducing its weight and making the device intrinsically compliant, characteristic that will be discussed in the following chapters [58, 63].

\subsubsection{The Active Leg Exoskeleton (ALEX by the University of Delaware):}

This device incorporates an extra DOF on the ankle compared with LOPES. Similar to the previous system, a force control strategy is implemented in order to achieve an assist as needed strategy. However, it does not present compliant actuation at its joints, instead a force field controller is used based on load cells connected to the linear actuators of the system $[5,6]$.

\subsubsection{Automated Locomotion Training using and Actuated Compliant Robotic} Orthosis (ALTACRO by The Vrije Universiteit Brussel):

This robotic device is the result of a research project contributing to the synergy between robotics and rehabilitation. The exoskeleton structure has 8 powered DOF; 2 at the hip, 1 at knee and 1 at the ankle per leg [66]. The electrically powered actuators present intrinsic mechanical compliance 


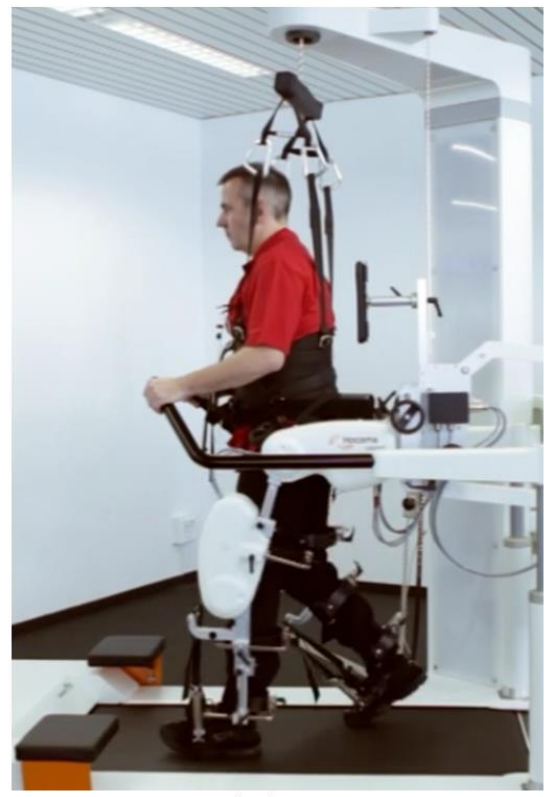

(a)

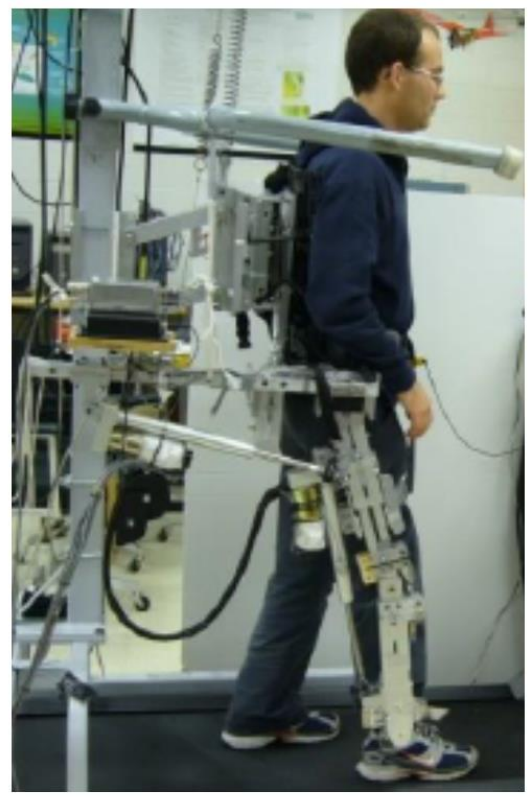

(c)

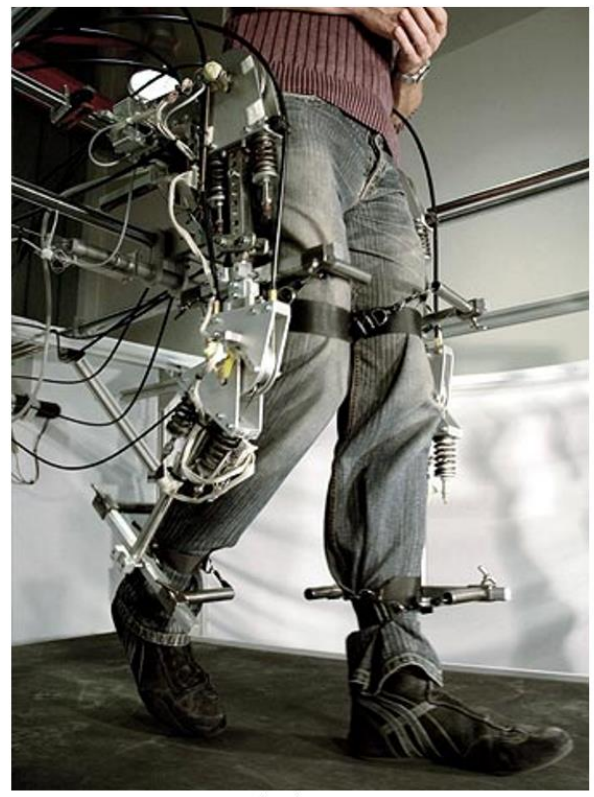

(b)

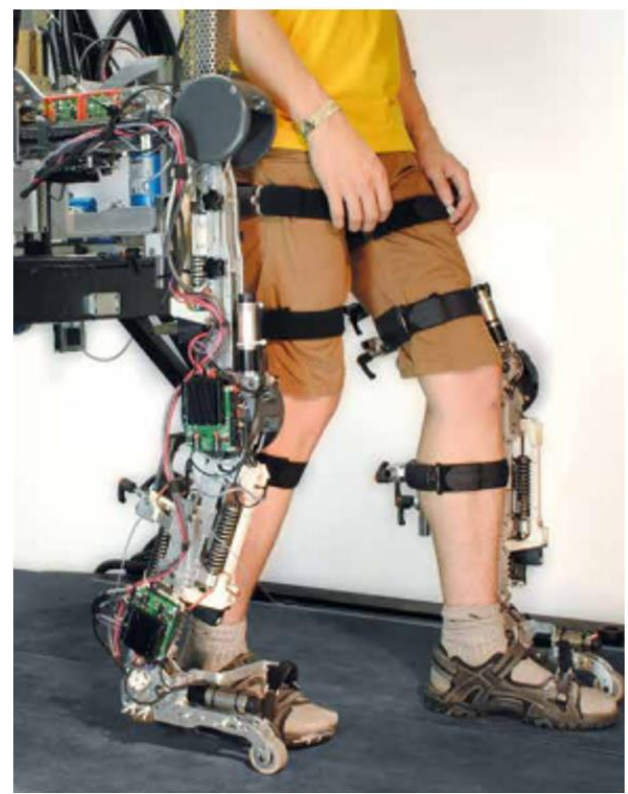

(d)

Figure 2-2. Treadmill-Based Exoskeletons. (a) Lokomat, (b) LOPES, (c) ALEX, (d) ALTACRO

by implementing compliant actuators to be discussed in the future sections [10]. The device is capable of force control and implements a gravity compensation functionality to assist the therapist during rehabilitation trainings with the patient. 


\subsubsection{Over ground-Based:}

This category comprehend devices that allow the patient to move over ground, not being constrain to a fixed location over a treadmill. They are subject to more challenges and limitations compared to the first sub-division and terms of autonomy, balance and energy. The following devices are characterized by the use of electric motors in combination with a gearboxes with large reduction ratio, required to achieve the necessary torque for their task. A brief review of the most relevant robotic devices in this category and pertinent comments about their functionalities and limitations are given below.

\subsubsection{ReWalk ${ }^{\mathrm{TM}}$ :}

This is a commercial robotic device from ARGO Medical Technologies Ltd. Presents actuation at hip and knee only in the sagittal plane and an unactuated ankle joint [3]. The exoskeleton weights approximately $23.3 \mathrm{Kg}$ and was the first FDA cleared exoskeleton for rehabilitation and personal use in the United States. The robotic system is not self-balanced, thus the user needs to provide the lateral stability assisted with clutches or walker.

\subsubsection{Rex:}

This commercial device developed by Rex Bionics based on New Zealand, weights around 40 $\mathrm{Kg}$ and is actuated at hip, knee and ankle. Is the only self-balanced exoskeleton available in the market, this makes it especially suitable for upper limb therapeutic exercises in stand position. Is currently undergoing clinical studies to evaluate its potential benefits; toward FDA clearance for rehabilitation in the United States [46]. Its size and weight are directly related to its ability to keep balance. A fixed gait pattern programed in the exoskeleton is intended to project the weight of the system into the area in contact with the ground; is recommended for regular hard surface.

\subsubsection{Ekso:}

It is a system designed and built at Ekso bionics, originally Berkeley Bionics [14, 34]. Similar to ReWalk, it is actuated at hip and knee by two linear electric motors, and clutches or walker are necessary to maintain balance. This device is more rehabilitation-oriented and is FDA cleared for rehabilitation in the United States

\subsubsection{Indego:}

This device is the latest exoskeleton with FDA cleared for personal and rehabilitation use. It presents a light and modular design with hip and knee actuation; with a weight of approximately 12 $\mathrm{Kg}$ it is the lightest powered exoskeleton system in the market [42]. Originally developed at Vanderbilt University and partnered with Parker for commercialization, it is now known as the Indego 
Exoskeleton. It is actuated only at the sagittal plane, thus the stability is once again up to the user. The joints in the exoskeleton present significantly less torque than the other devices, however a combination on the user stability and normally active brakes at the knees [16], allows the user to stand and walk with the exoskeleton.

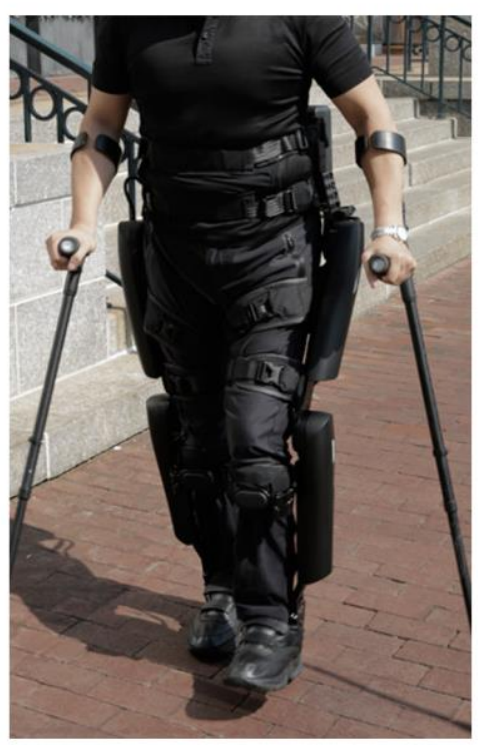

(a)

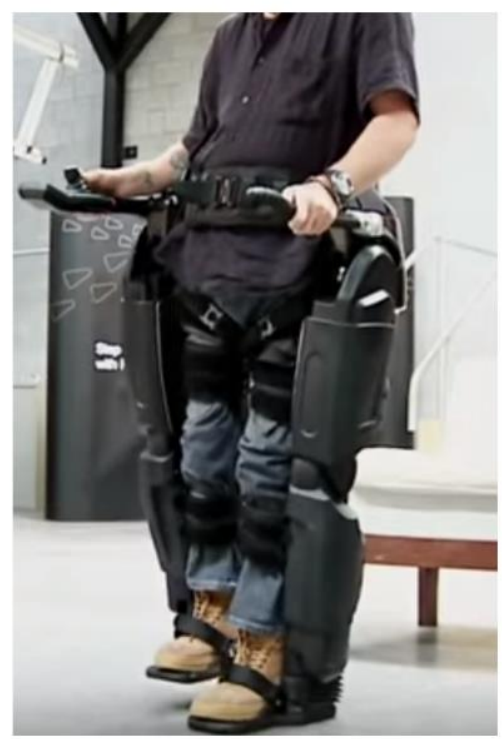

(b)

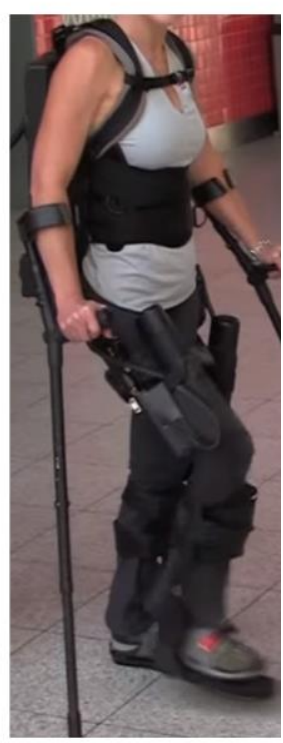

(c)

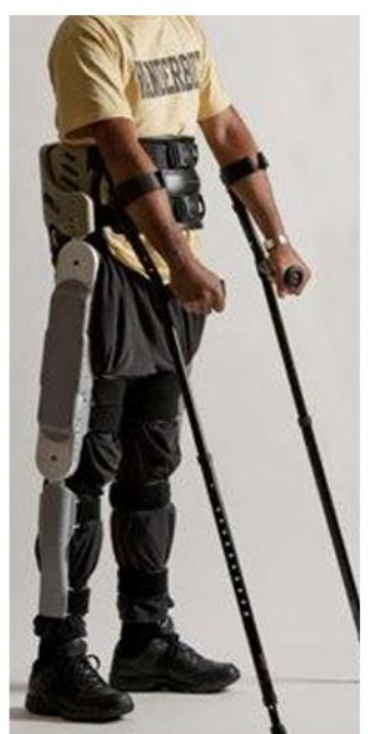

(d)

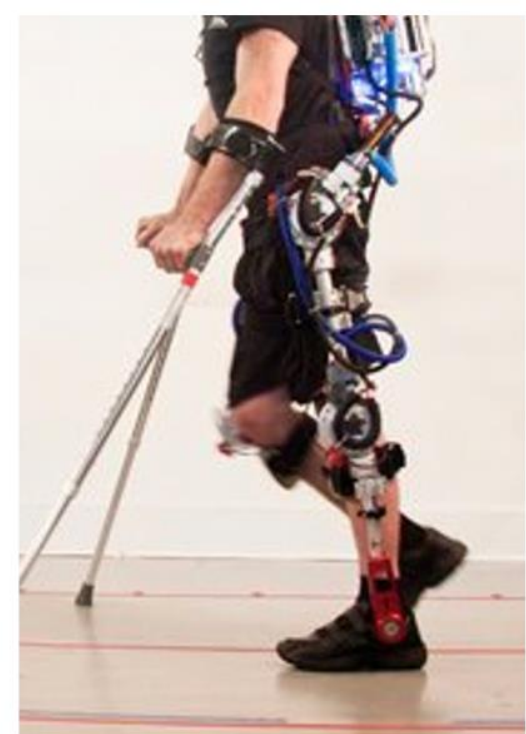

(e)

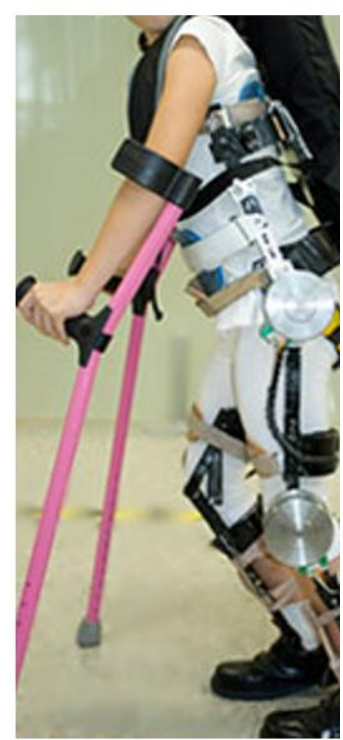

(f)

Figure 2-3. Over Ground-Based Exoskeletons. (a) ReWalk ${ }^{\mathrm{TM}}$,(b) REX Bionics, (c) Ekso Bionics, (d) Indego, (e) X1-Mina Exoskeleton, (f) ATLAS Lower Limb Exoskeleton. 


\subsubsection{NASA-IHMC X1 Mina Exoskeleton:}

NASA Johnson Space Center and IHMC are jointly developing a powered lower extremity exoskeleton. The focus of this collaboration is to develop a robotic device for a range of applications, including mobility assistance for abled and disabled users, rehabilitation, and exercise [29]. This system have had several prototypes, from the original IHMC Exoskeleton with several compliant DOF [32], to the latest version with hip and knee actuated, and passive joints at hip and ankle [29, 40]. Incorporates torque control at the actuated joints and similar to most of the systems in this section, is not capable of maintaining balance.

\subsubsection{ATLAS 1st Prototype:}

The ATLAS exoskeleton is a device resulting partially from this work at the Centre for Automation and Robotics (CSIC-UPM), its technology has been licensed to Marsi Bionics [36]. The device is actuated at hip, knee and ankle and is intended to assist children with walking disabilities. Differently from many of the current existing robotic systems, the $1^{\text {st }}$ prototype of the ATLAS exoskeleton was originally intended for quadriplegic users, thus actuation at the ankle and a special walker[8] were required in order to achieve lateral balance [19, 20, 50]. A predefined gait trajectory is implemented at the joints of the exoskeleton, based on walking data from healthy subjects. By sensing the force at the foot of the exoskeleton in contact with the ground, online adaptations to the gait can be done in order to adjust to the user walking variabilities. The work developed during this thesis, aims to produce compliant actuators that can be incorporated into the exoskeleton in order to allow adaptability among other advantages to the predefined gait.

\subsubsection{ATLAS project- challenges}

Human-robot-environment interactions are some of the main aspects to consider in the field of robotic exoskeletons, particularly with those intended for rehabilitation or medical purposes. Actuators for empowering robots should have low mechanical impedance or backdriveability to allow the robot's adaptation to the environment and to the human. Understanding mechanical impedance as the minimum amount of force an actuator outputs for a given load motion, can be thought of as the stiffness of the actuator output [48].

An actuation system can be seen as a complex of three parts: power source, actuator, actuation mechanism. Due to the mobility necessity of exoskeletons and the need to carry their own power source and control hardware [34] (except treadmill-based exoskeleton robots) new generation of legged empowering robots required large power to feed both the robot (increase speed) and the payload (increase force). Because autonomous empowering robots carry their own actuators, an increase of 
actuator weight would rely on an overall increase in robot weight, which in turn will require larger power for a given performance.

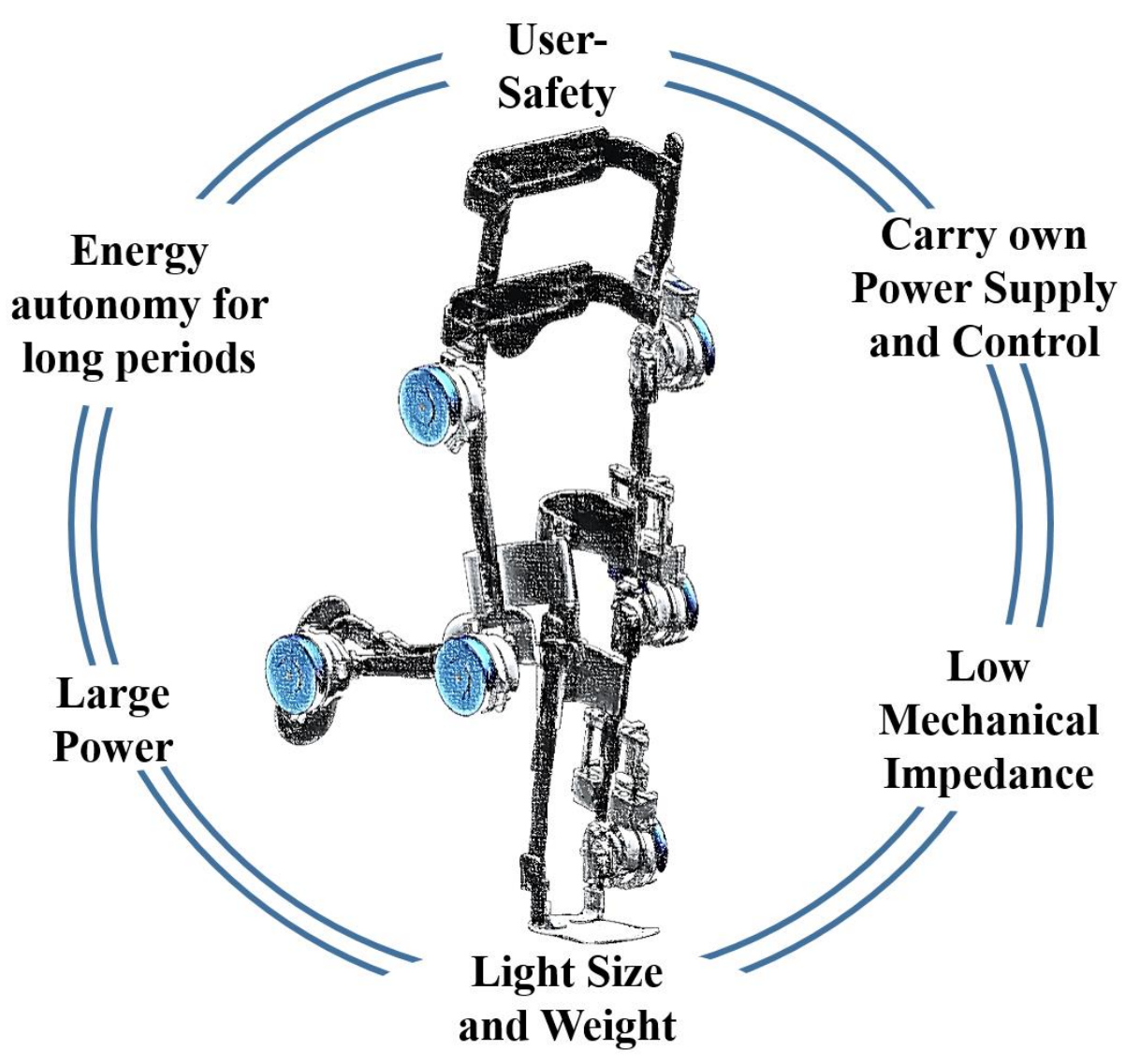

Figure 2-4. Challenges in Rehabilitation Exoskeletons' Design.

Ultimately, exoskeletons will be worn by users that should be able to handle then and be satisfied with their aesthetically appearance. Therefore, small size and reduced weight are required for exoskeleton applications. The size reduction avoids the problem of bothering the regular activities of the user (such as sitting or passing through a door), and by having lower dimensions, the associated inertia that the actuators must resist decreases. The design of a light weight, energy efficient and powerful source is another challenge. Battery is a common power source, however considering that the current state of the art in high energy-density power supplies is still immature, one alternative solution is to optimize the power consumption of the robot itself.

This work will focused particularly in robotic exoskeletons for over-ground applications. A brief comparison is given in Table 2-2 between the current devices in the state of the art. The 
highlighted cells correspond to the improved prototype of the ATLAS exoskeleton, provided with compliant joints in its structure that constitutes an advance beyond the current SoA.

Size and weight are main concerns in exoskeleton applications, for these the robotic devices should present light, and compact structures. The emulation of a more natural actuation could lead to robotic systems with lower energy consumption as well as more adaptability to the user conditions an environment. To achieve this, an understanding of the human biomechanics during locomotion is required, and it this matter will be addressed in the next chapter.

Table 2-2. Basic Comparison of Over-Ground Exoskeletons.

\begin{tabular}{|c|c|c|c|c|c|c|}
\hline $\begin{array}{c}\text { Exoskeleton } \\
\text { Device }\end{array}$ & $\begin{array}{c}\text { Weight } \\
(\mathrm{kg})\end{array}$ & Balance & $\begin{array}{l}\text { Users } \\
\text { hight } \\
\text { (cm) }\end{array}$ & $\begin{array}{c}\text { Actuated } \\
\text { DOF }\end{array}$ & Gait & Actuation \\
\hline ReWalk $^{\mathrm{TM}}$ & 23.3 & $\begin{array}{l}\text { User-Cluthes or } \\
\text { walker }\end{array}$ & $\begin{array}{l}\text { 160-190 } \\
\text { (Adults) }\end{array}$ & 2 per Leg & $\begin{array}{c}\text { Predefined-Fix } \\
\text { Based on healthy } \\
\text { subjects - NO ankle }\end{array}$ & $\begin{array}{c}\text { Stiff-Rigid } \\
\text { Compliant } \\
\text { Ankle }\end{array}$ \\
\hline Rex Bionics & 40 & Self-Balanced & $\begin{array}{l}\text { 160-190 } \\
\text { (Adults) }\end{array}$ & 5 per leg & $\begin{array}{c}\text { Predefined-Fix } \\
\text { Addapted for static } \\
\text { stability }\end{array}$ & Stiff-Rigid \\
\hline Ekso Bionics & 20 & $\begin{array}{l}\text { User-Cluthes or } \\
\text { walker }\end{array}$ & $\begin{array}{l}\text { 158-188 } \\
\text { (Adults) }\end{array}$ & 2 per Leg & $\begin{array}{c}\text { Predefined-Fix } \\
\text { Based on healthy } \\
\text { subjects - NO ankle }\end{array}$ & Stiff-Rigid \\
\hline Indego & 12 & $\begin{array}{l}\text { User-Cluthes or } \\
\text { walker }\end{array}$ & $\begin{array}{l}\text { 155-191 } \\
\text { (Adults) }\end{array}$ & 2 per Leg & $\begin{array}{c}\text { Predefined-Fix } \\
\text { Based on healthy } \\
\text { subjects - NO ankle }\end{array}$ & $\begin{array}{c}\text { Stiff-Rigid } \\
\text { Compliant } \\
\text { Ankle }\end{array}$ \\
\hline NASA-X1 & -- & $\begin{array}{l}\text { User-Cluthes or } \\
\text { walker }\end{array}$ & $\begin{array}{l}\text { 155-191 } \\
\text { (Adults) }\end{array}$ & 2 per Leg & $\begin{array}{c}\text { Predefined-Fix } \\
\text { Based on healthy } \\
\text { subjects - NO ankle }\end{array}$ & $\begin{array}{c}\text { Stiff-Rigid } \\
\text { Compliant } \\
\text { Ankle }\end{array}$ \\
\hline ATLAS & 10 & $\begin{array}{c}\text { User-Cluthes or } \\
\text { Walker } \\
\text { Self-Balance -Special } \\
\text { Walker }\end{array}$ & $\begin{array}{c}\text { 130-165 } \\
\text { (Children) }\end{array}$ & 3 per Leg & $\begin{array}{l}\text { Predefined-Fix } \\
\text { Based on healthy } \\
\text { subjects - WITH } \\
\text { ankle }\end{array}$ & Stiff-Rigid \\
\hline
\end{tabular}




\section{Chapter 3}

\section{Human Gait- the Biomechanics of Locomotion.}

The research on the mechanism of human locomotion can supply important enlightenment to the design of powered gait exoskeletons. By analyzing the biomechanics of gait, it is possible to get a better understanding of the issue we are dealing with and to obtain the bases for possible solutions to the main barriers and limitations in the walking assistance field. For the design of orthoses and exoskeletons it is particularly useful to know the requirements of each one of the involved joints, in terms of power, kinematics, and functionality of each of them. Conventionally, the human leg can be modeled as a system of 7 degrees of freedom [12]. In Figure 3-1, the human anatomic planes are presented, where the human leg presents three rotations in the sagittal plane corresponding with flexion and extension of hip, knee and ankle; two rotations in the coronal plane and the remaining two in the transverse plane, all four for hip and ankle.

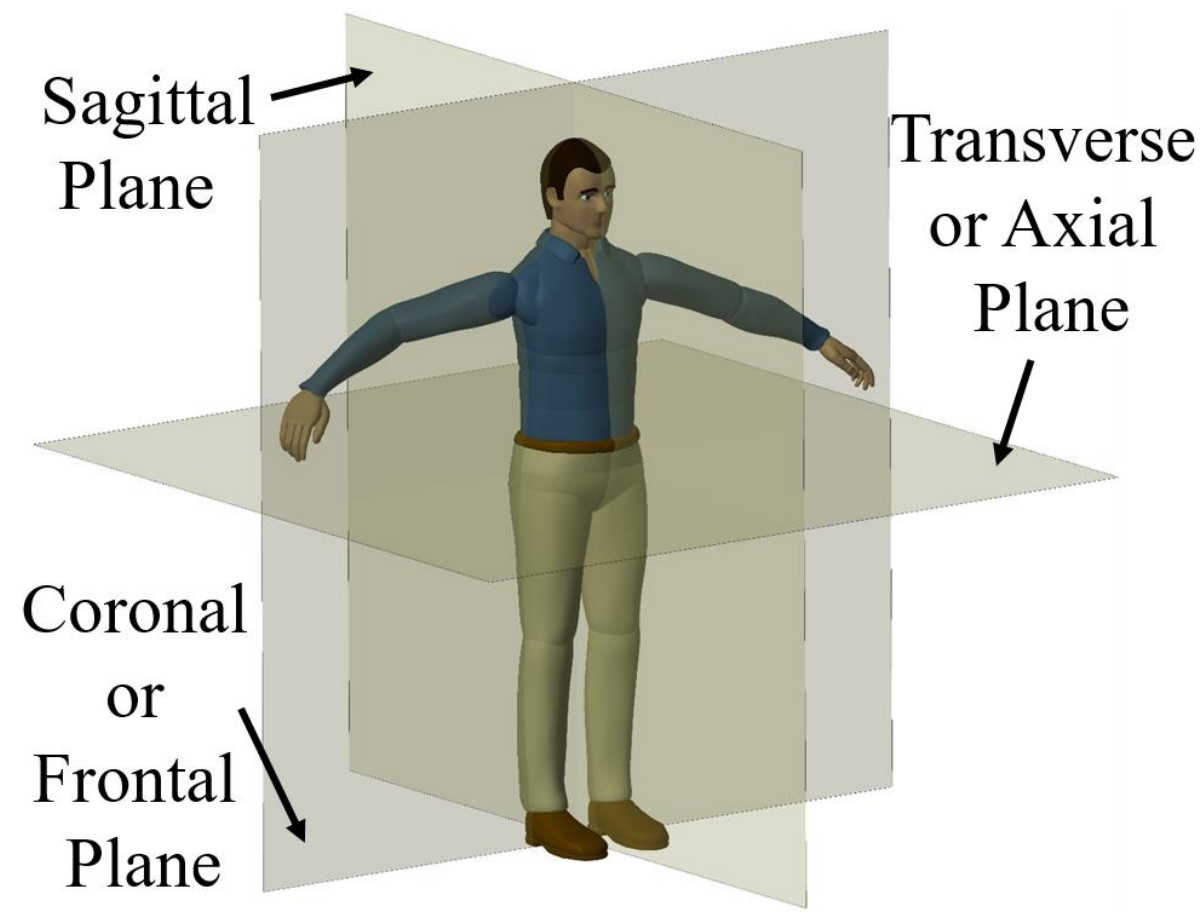

Figure 3-1. Planes of Section of the Human Body. 
Robotic exoskeletons tend to present semi-anthropomorphic structures, designed to match human joints. However, they cannot exactly replicate the human joint kinematics due to its complex nature. Exoskeleton's design should be kinematically compatible while still providing satisfactory locomotion functionality [23]; this chapter intents to analyze the biomechanics of locomotion in order to integrate the relevant elements into the actuation mechanism of robotic exoskeletons for a more optimal and natural operation.

\subsection{Walking-based on clinical gait analysis}

Walking is a complex action, it is the results of synergic movements of skeleton around the joint and powered by several rhythmic muscles contraction. With proper instrumentation the movements, and biomechanics of the body, as well as the activity of the muscle, it is possible to analyze the kinematics and dynamics of the human walk. This research work is towards a special branch of biomechanics called gait analysis [23]. The sagittal plane is the predominant in human locomotion [12] and therefore studies of gait focus on it; Figure 3-2 presents a Clinical Gait Analysis (CGA) pattern, on top, where angular variation in the sagittal plane of the hip, knee and ankle joints of the leg are shown during a locomotion cycle. The locomotion cycle is divided into three main phases that can be seen in the bottom of the figure, corresponding to the heel-strike, toe-off and swing of one leg. Walking is often modeled as the motion of two coupled pendula, because the stance phase behaves like an inverted pendulum moving about the stance foot, and the swing phase behaves like a regular pendulum swinging about the hip [59].

A level-ground gait cycle is typically defined as beginning with the heel strike of one foot and ending at the next heel strike of the same foot [4]. Walking kinematics can slightly vary between subjects, nevertheless there are characteristic points that allow a division of the gait cycle into the support phase ( $60 \%$ of a gait cycle) and the swing phase ( $40 \%$ of a cycle). The swing phase represents the portion of the gait cycle when the foot is off the ground. The stance phase begins at heel-strike when the heel touches the floor and ends at toe-off (approx.at $62 \%$ of the cycle) when the same foot rises from the ground surface.

The phases of the locomotion cycle can be sub divided as follows:

- Initial contact $(0 \%)$ : The heel gets in contact with ground. Double support phase begins.

- Loading response ( $0 \%$ to $12 \%)$ : In this sub phase the leg accommodates to the ground and the body weight. Double support ends. 
- Mid stance (12\% to 30\%): Single support begins, the body rolls over the foot like an inverted pendulum

- Terminal support (30\%to50\%): The leg contributes to the ground clearance of the leading leg, maximum high of the Center of Mass of the body.

- Pre-swing (50\% to 60\%): Toe-off impulse finishes and the leg lift the ground.

- Swing phase- Initial swing: After the acceleration provided, the foot is lifted to avoid the ground.
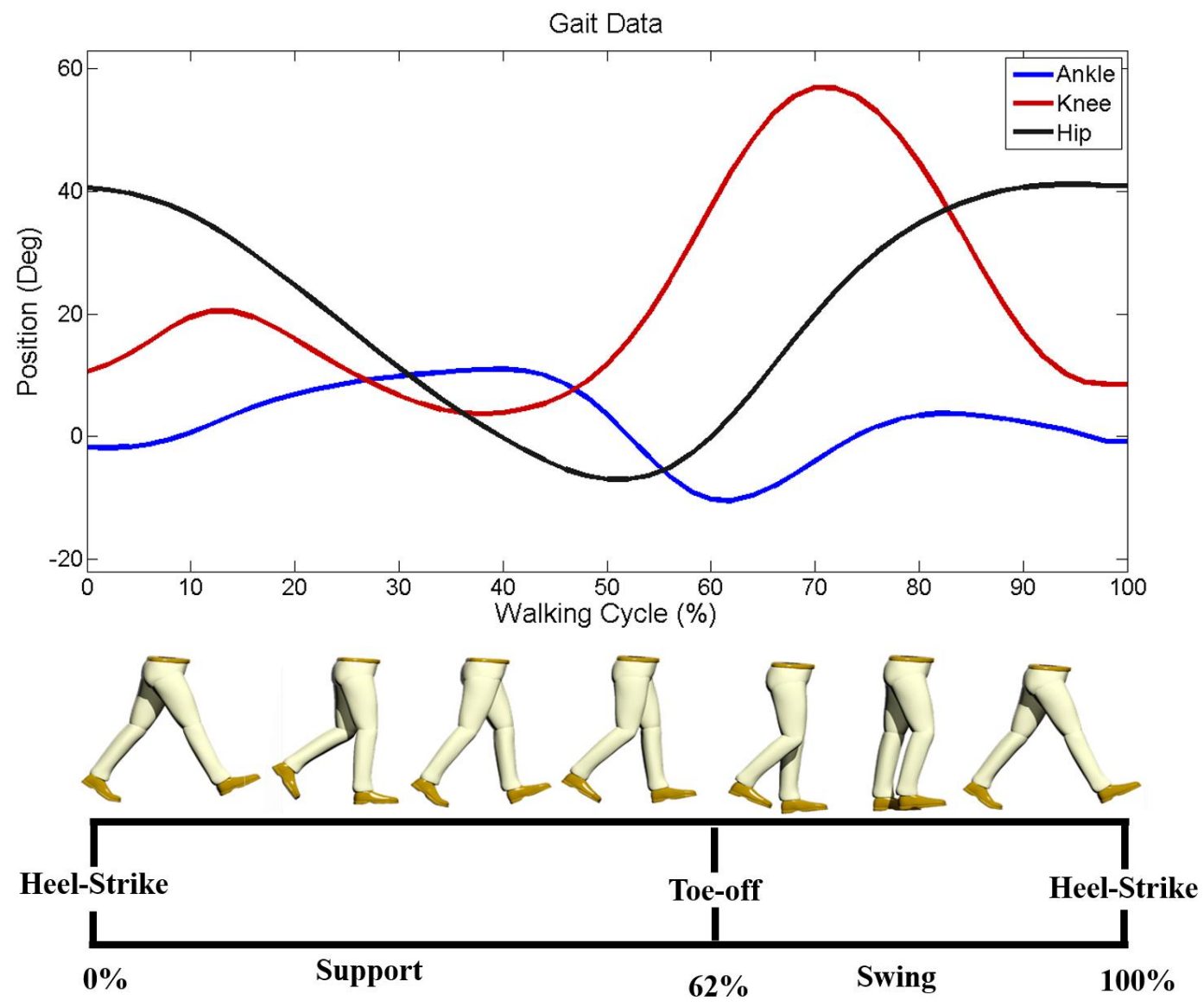

Figure 3-2. Data from Clinical Gait Analysis (CGA) and Phases of the Locomotion Cycle- Right Leg.

- Swing phase-Mid swing: The leg advances with the knee flexed, like a direct pendulum, preventing stumbling.

- Swing phase-End swing: the leg goes from a flexed knee in the toe-off to a fully extended knee prior to the heel-strike. In a dynamic walk, the knee motion is achieved by taking advantage of the hip movement and leg inertia. The kinetic and potential energy can be used to extend the knee with a little to no-actuation energy required. 
In Figure 3-3 the normalized mean data taken from a clinical gait analysis with 16 children [53] are shown at self-selected normal speed $(\sim 0.6 \mathrm{~m} / \mathrm{s})$. Although, energy and power in the gait are speed dependent, the figure below can be still used to illustrate the behavior of the joints during the locomotion cycle.

It can be noticed the synergy between the acting forces [69]. These patterns of energy transfer generation and absorption by the muscles and through the joints are quite complex. However, some generalized observations can be made. It is evident that the major contributor at push-off is the ankle (near 50-60\% off the cycle). At toe-off the energy increase comes from the hip flexors and across the hip joint. As the leg accelerates forward, there is a flow of energy from the trunk through the joints with no muscle activity. Initial deceleration of the leg results from energy absorption by the hip extensors and knee flexors. The final deceleration involves a passive flow of energy from the foot and shank to the thigh and trunk [69].

Many implementations of gait control can be performed by analyzing the energy flow; in a simulation study [51] the dynamic principles of the human gait are exploited to implement an economic gait control, resulting in a reduction of simulated energy consumption when different elements such as dampers, brakes or springs act at the joints in different phases of the locomotion cycle as required.
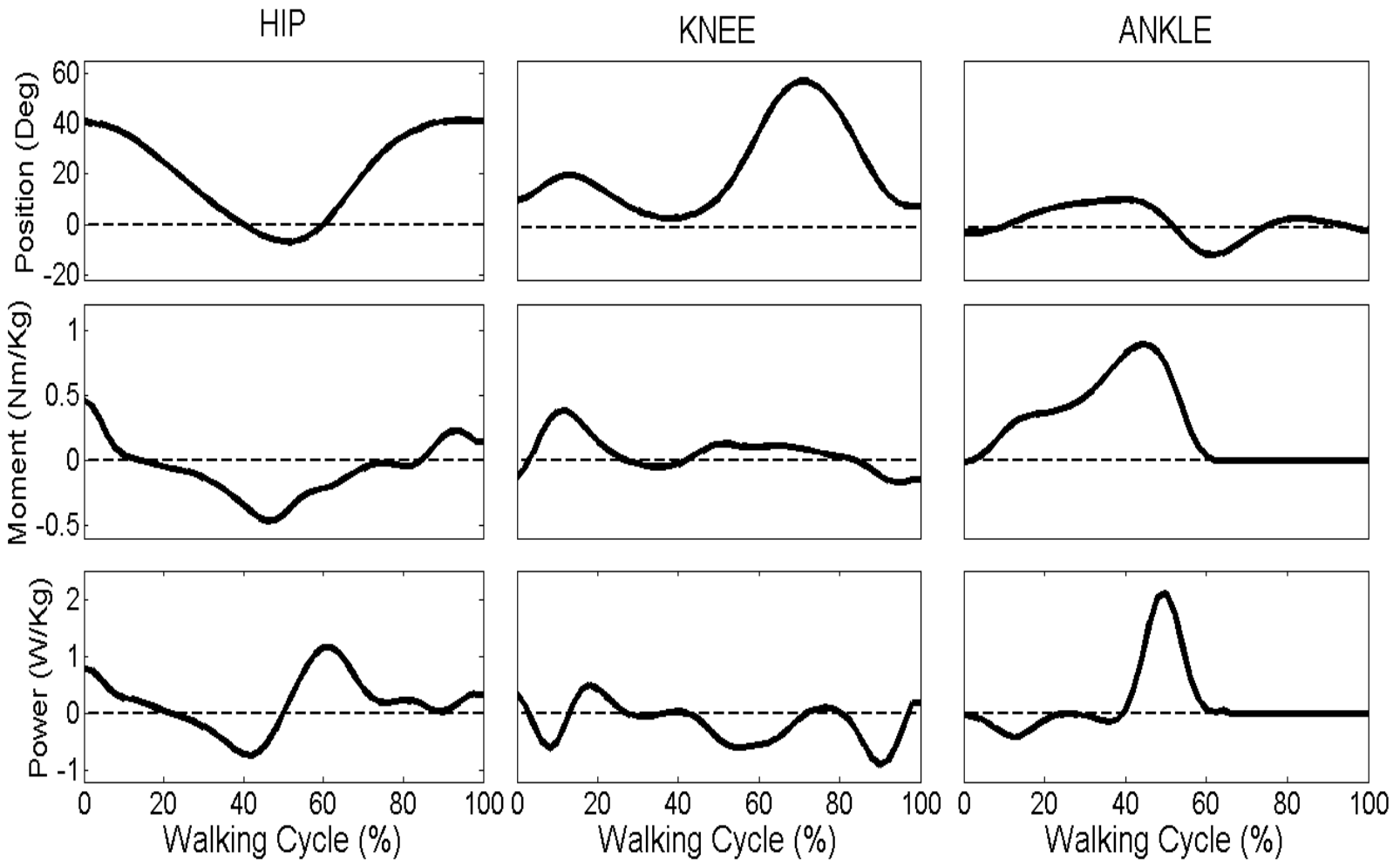

Figure 3-3. Clinical Gait Data. Angular Position, Moment, and Power of the Sagittal Joints [53]. Walking Cycle Moderate-Speed. 


\subsection{Natural Actuation- The Muscle complex}

Humans are capable of producing autonomously a wide range of stable movements in environments with unpredictable disturbances. Dynamic control of joint stiffness is crucial for humans to adapt to changes in environmental conditions [38]. Muscles in biological systems modulate the stiffness and position of the joint achieving high adaptability, force transmission and significant reduction of energy expenditure [15].

Muscles and tendons are responsible for humans to excel the performance of the best robots when physical interaction with the world exists, especially interaction that involves impacts or kinetic energy transfers [24]. The natural muscle provides a wide range of adaptability and is capable of generating, absorbing or transferring energy. The high power-to-weight ratio, force-to-weight ratio, compliance, and control of muscle, when compared with traditional robotic actuators, are the main barriers for the development of machines that can match the motion, safety, and energy efficiency of human beings or animals.

\subsubsection{Natural-Muscle}

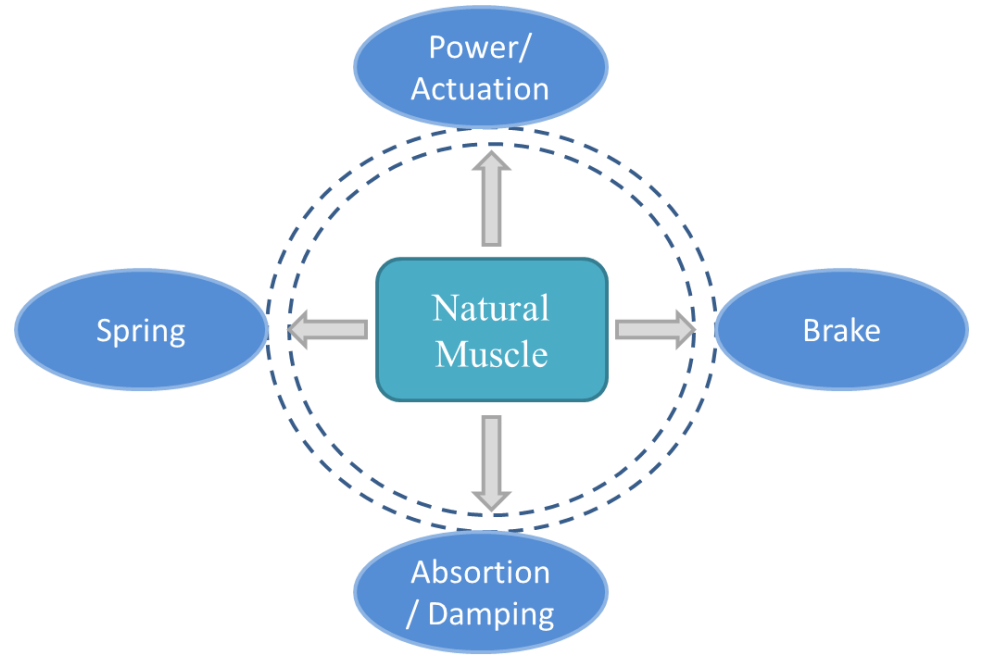

Figure 3-4. Multifunction of the Natural Muscle.

The mechanical structure of the muscle can be described by the following simplified parts: muscle fiber, tendon, aponeurosis and the connective tissue. In the classic Hill-Muscle Model, presented in Figure 3-5 the tendon and aponeurosis have elastic properties with a nonlinear stressstrain behavior and are described as a serial elastic component (SE). The muscle fibers are surrounded by connective tissue and the mechanical properties of these structures can be described by a parallel elastic component (PE). In combination with the contractile component $(\mathrm{CC})$ with inherent damping 
properties, the functioning of a muscle can be described technically. Some important features are well reported in literature: several studies emphasize the importance of the force-velocity relation for the stabilization of movements, and the elastic properties of muscles are relevant for energy efficient locomotion, and shock protection [21].

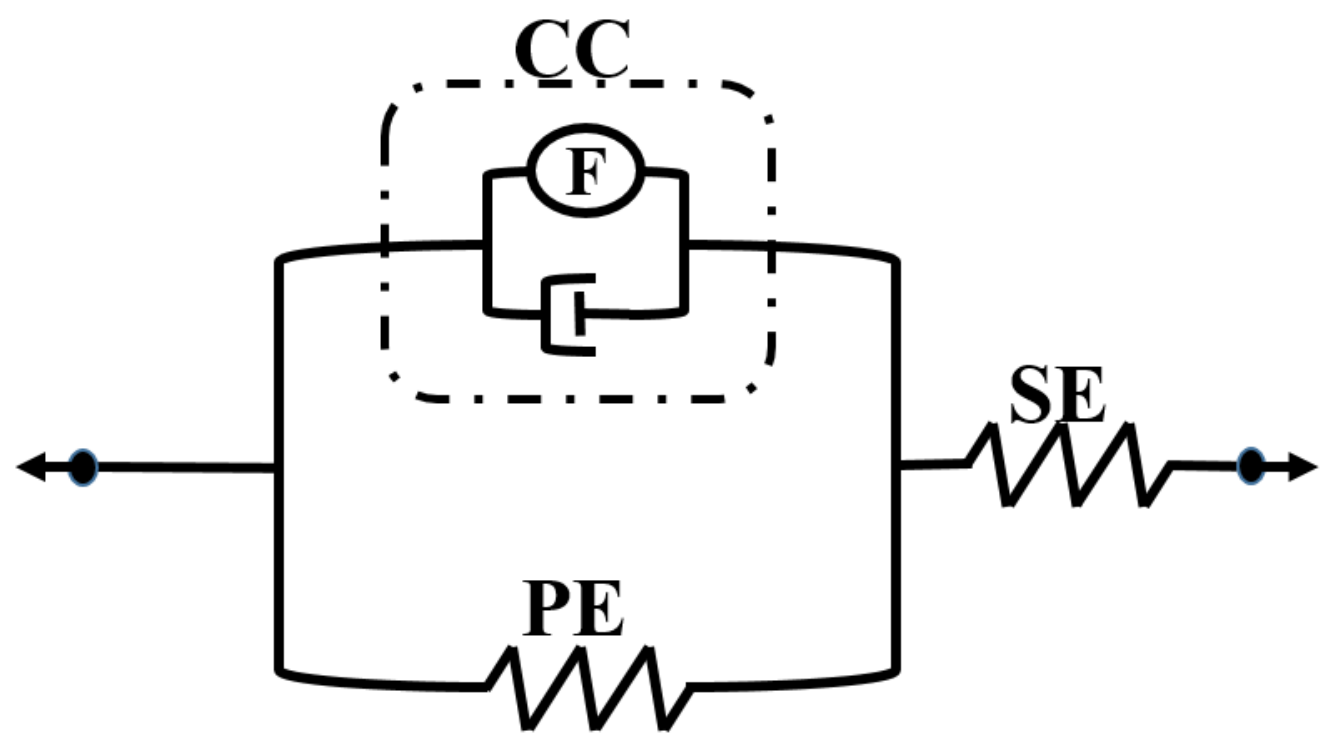

Figure 3-5. Classic Hill-Muscle Model

In biology, joint motion takes place by the activation of antagonistic muscles because muscles can only exert a pulling force and cannot return into their original positions. Joint compliance is a function of muscular co-contraction given that an increase in a muscle's activation increases the number of parallel elastic elements used by the muscle, which raises the muscle's stiffness. To emulate this behavior, the elastic elements must have a nonlinear force-length relationship [38]. This allows the joint to have a great range of variable rigidity, if both muscles act at the same time an isometric action (without motion) takes place and the joint achieves high rigidity, if only one muscle is activated the joint will be moved to one side, if both muscles are deactivated the joint will show a free motion only with the rigidity of the tissues that compose the muscles. In the motion of a complex joint, not only a pair of muscles with their associated tendons is activated; a group of muscle and tendon units (MTU) are involved and act redundantly and synergistically, MTU are capable of storing energy and transferring it to the other joints and then reuse it.

When looking at the complexity and variety of movements that biological muscles can generate, it is shown that biological muscle is a versatile, powerful, and flexible actuator. Muscles are 
said to have benefits for the generation and the control of biological movements compared to classical actuators. They are thought to operate energy efficiently, have intrinsic properties that have inherently stabilizing effects, and reduce the control effort. This is achieved because muscles can operate in different modes depending on the loading situation and the structural implementation [21], also observed in the power behavior shown in Figure 3-3. In active exoskeletons, the main power must be provided by the actuators, taking aside passive elements. Commercial devices nowadays present joints actuated with stiff actuators, which result in devices that cannot adapt to unpredictable environments, consume more energy and are not appropriate for human machine interaction. A key difference of these systems is the lack of compliance or spring-like behavior found in biological systems [2].

The primary limitations of implementing active compliance in robot joints are feedback control constraints and stabilization problems [39]. Simple passive compliance can be achieved by inserting an elastic component between the actuator and the end-effector. In this case, the need for feedback is removed, but the joint stiffness becomes a constant function of the mechanical elements used in the robotic device and is therefore not controllable. A more advanced version of this implementation uses variable stiffness elastic elements to dynamically control joint compliance [38]. By adjusting the stiffness of the systems during operation, better adaptation the environment and shocks is possible. The adjustments on the joint stiffness can allow the emulation of certain properties, and characteristics of the muscle, such as elasticity, energy storage, and torque generation with reduced power requirements.

\subsection{Joint Analysis}

Focusing in the design of powered exoskeletons and based on the data collected by the clinical gait studies, in combination with an understanding of the muscle complex, the different behaviors that each joint should fulfill along the locomotion cycle in order to achieve a safe, and energy efficient human-like gait are discussed below.

\subsubsection{Hip}

From Figure 3-2, and Figure 3-3 it can be appreciated that the hip joint presents a sine wavelike pattern. This cyclic movement, could benefit from elastic elements connected in series to the joint in order to reduce power peaks [68]. The range of motion of angles of the hip joint during walking is from about $-20^{\circ}$ to $40^{\circ}$. There are positive values and negative values in the joint torque of the hip joint, which indicates the need of a bi-directional actuator. Negative extension torque is required in early support as the hip supports the load on the leg. Hip torque is positive in terminal support and 
initial swing as the hip propels the leg forward during swing. In late swing, the torque turns to negative as the hip decelerates the leg prior to heel-strike [23]. As shown in Figure 3-3 there is positive work and negative work of the joint power of hip joint in walking. And in the supporting phase, the body is pushed forward by the energy which is released during toe-off phase. The two peak power values appear respectively at the time of heel-strike and toe-off. As the average power is positive, the hip joint plays an important role in the human gait.

Figure 3-6 (a) presents the relationship between torque and angle for the hip joint during the locomotion cycle from the data presented in [53]. This relationship is related to the impedance of the joint, sometimes also discussed as joint stiffness. It can be noticed two pronounced semi-vertical lines at the ends of the plot, indicating the generation of power. High torque generated in both directions

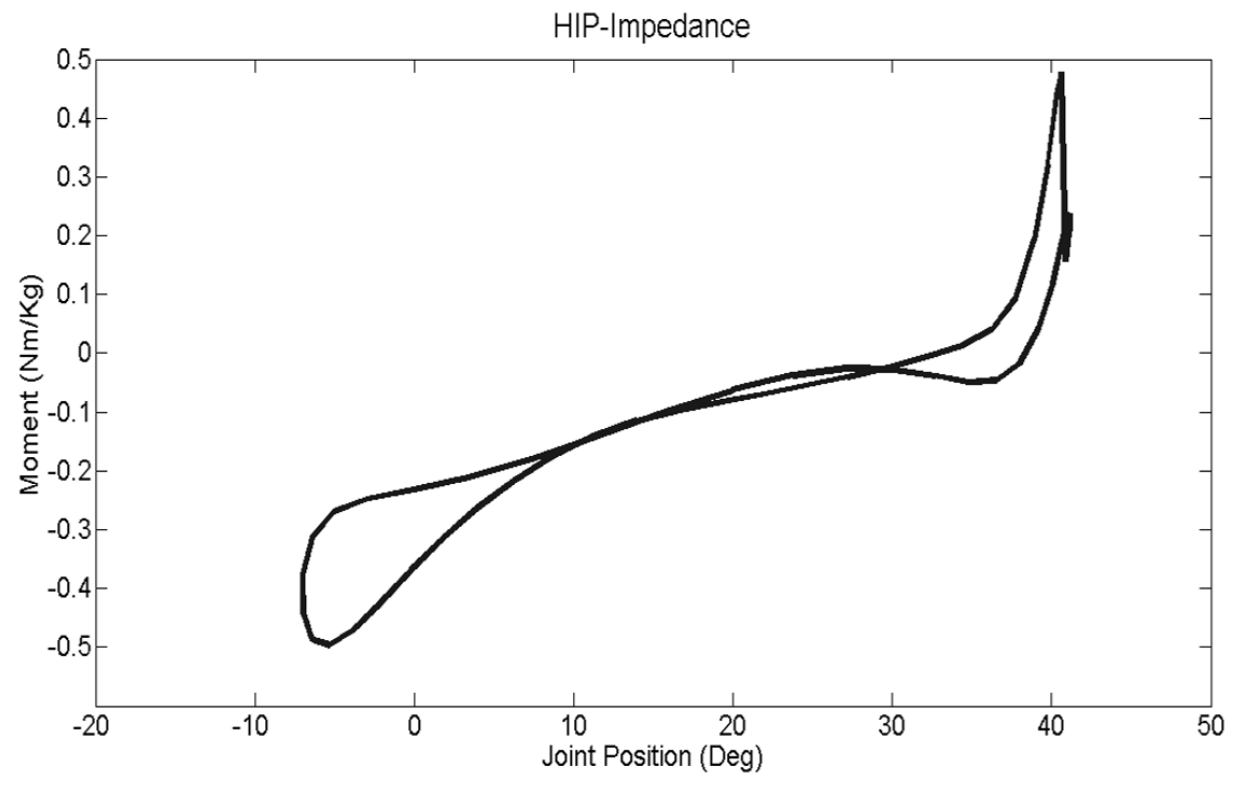

(a)

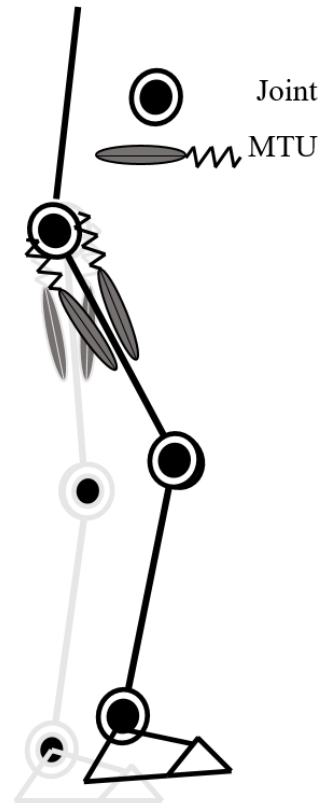

(b)

Figure 3-6. (a) Hip-Impedance and (b) Hip-Leg Model to Fulfill Hip Requirements.

along the walking cycle, the hip is the primary joint that makes walking possible. Based on the behavior of the natural muscle, in Figure 3-6 (b), a simple actuation configuration to fulfill the hip requirements is presented. Two muscle-complex in series with an elastic component attached to the hip to achieve bidirectional-motion. 


\subsubsection{Knee}

The knee is a multifunctional joint with a RoM of about $0^{\circ}$ to $60^{\circ}$, presenting different tasks during the locomotion cycle. Along the walking cycle, the knee power experiments several variations from power generation to power absorption, this makes complicated and energetically expensive to replicate the natural motion at the knee with traditional actuators. The human muscle is capable of adapting and absorbing energy without the need of dissipating it, instead this energy can be stored or transferred to other joints.

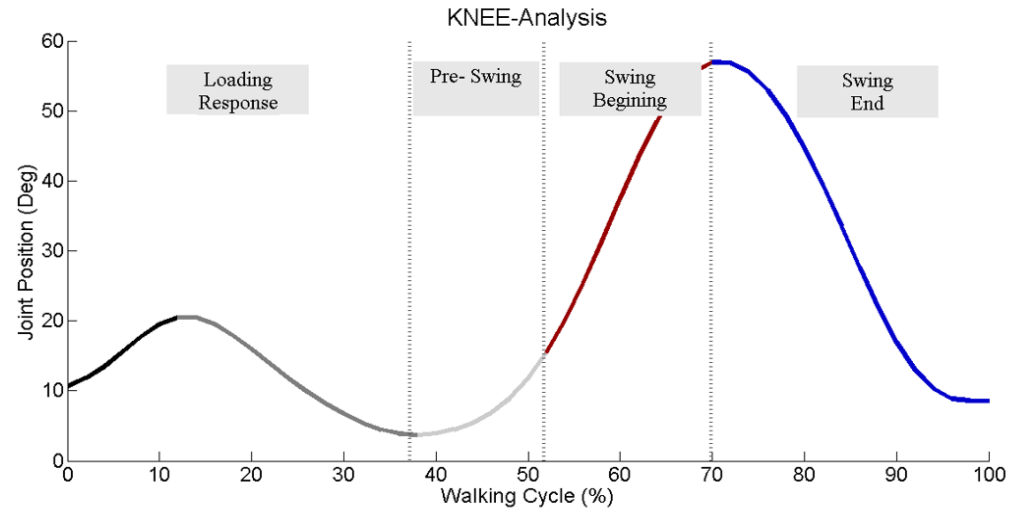

(a)

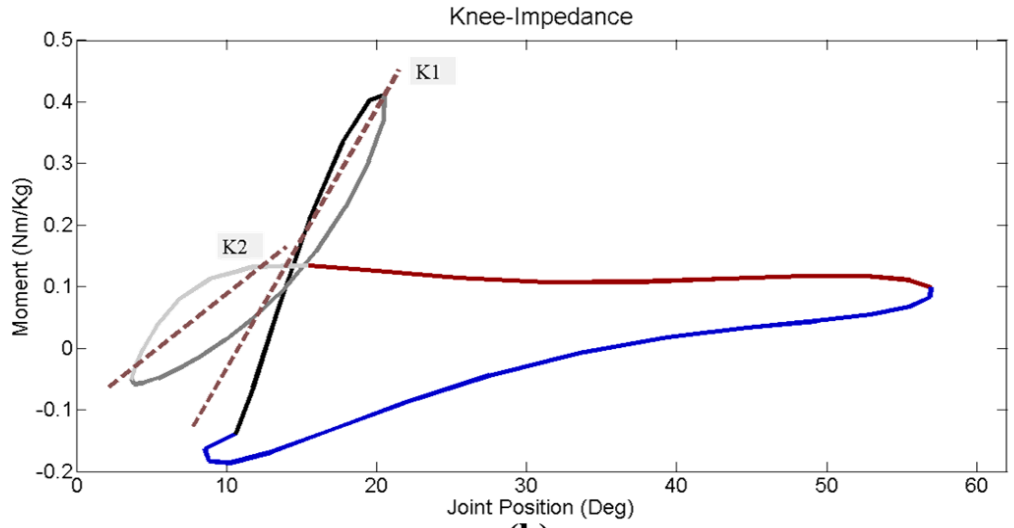

(b)

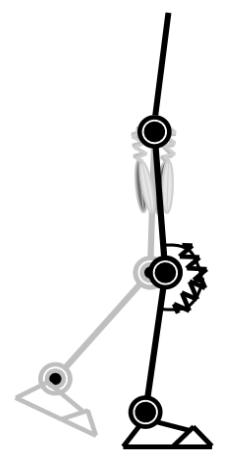

(c)

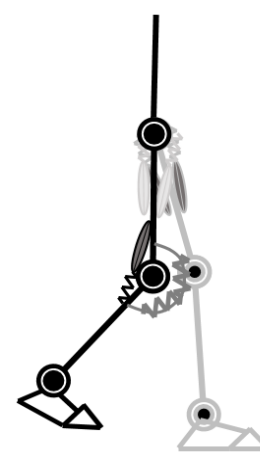

(d)

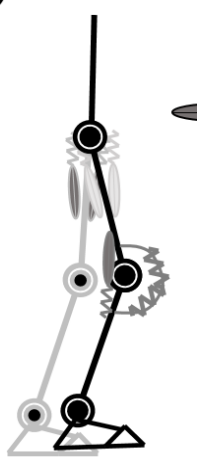

(e)

Figure 3-7. Knee Analysis. (a) Sub-Phases at the Knee During Gait. (b) Knee-Impedance Evolution During Gait. (c-d-e) Knee-Leg Model to Fulfill knee Requirements. 
During the support phase, the knee absorbs a certain amount of energy during flexion and generates as much as the same amount of energy for its extension, this load response occurs between 0- 40\% of the cycle as can be seen in Figure 3-7 (a), and Figure 3-3. When analyzing the variation of impedance at the knee along the walking cycle, it can be seen in Figure 3-7 (b) that the load response behaves similar to an elastic element acting and its corresponding return to the equilibrium position following a trajectory in presence of hysteresis. In this section of the gait, the knee should behave as a spring with a constant $\mathrm{K} 1$ according to the weight acceptance and the ground itself. Followed by the loading response, the knee flexes again just before toe-off during pre-swing. The spring constant at this stage should be lower than the prior section, indicating the ability to tune the spring constant. Figure 3-7 (c) presents the model of the leg, with a variable rotational spring that should be capable of fulfill the knee moment and motion of about $60 \%$ of the cycle in a dynamic walk. The energy absorbed during pre-swing can be used in another joint or stored for a later stage of the gait.

The beginning of swing, requires the knee to complete the flexion to assist the ground clearance. The power required from the knee to achieve the flexion is low, as only the inertia of the foot and shank needs to be moved, and in dynamic and fast walks, the kinetic energy of the locomotion can assist this flexion. An active actuation at the knee is required to supply the necessary power and hold the position as long as it is needed. At slower gaits, this actuation gets more relevant because there is not kinetic energy contributing, instead the weight of the limb oppose the flexion. In Figure 3-7 (b) and (d), a flat line (red-line) in the impedance plot and an active MTU connected to the knee indicate the presence of an active actuation at the joint with the rotational spring not acting, indicated by the dimmed color.

During the end of the swing phase, the leg transitions from a flexed knee to a fully extended knee prior to heel-strike. This knee motion is achieved by utilizing the hip movement and leg inertia. By reducing the impedance at the knee to a minimum, the kinetic and potential energy can be used to extend the knee without generating significant power at the joint. The energy stored at the pre-swing can also be used to successfully achieve the extension. Figure 3-7 (e) shows the leg model for this phase, a free joint (MTU and spring dimmed, not acting) could achieve the extension with an activation of the elastic elements and MTU at the end of the extension, to control or assist it.

\subsubsection{Ankle}

The ankle joint during the gait cycle produces positive and negative power, at normal walking speed it can be appreciated a significant positive power at the puss-off as can be seen in Figure 3-8 (a). This power generation occurs in a very small instant, similar to an impulse force, and represents the 
main complication when designing ankle joints for exoskeletons and prosthetic devices. The RoM at the ankle is about $30^{\circ}$ from approximately $-15^{\circ}$ to $15^{\circ}$, and during the support phase during a dynamic walk it can store part of the energy required during the push off by adapting to the ground and the weight of the subject.

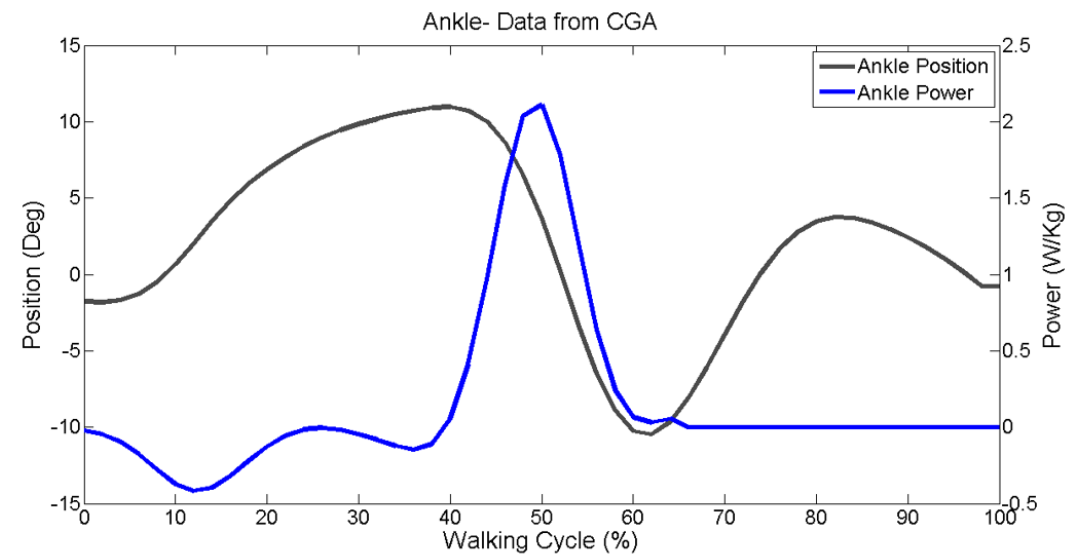

(a)

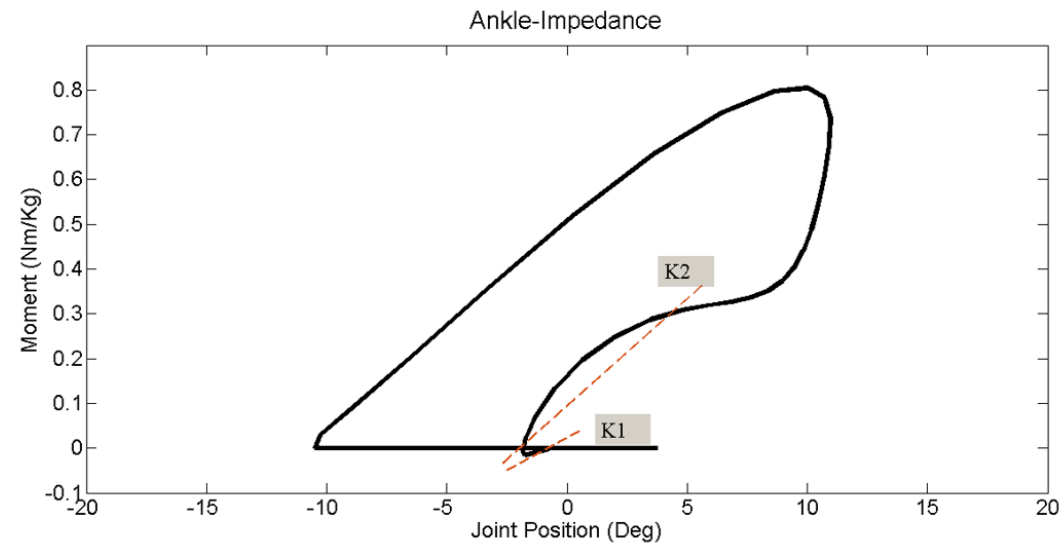

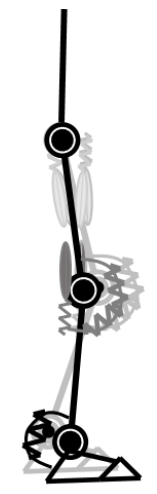

(c)

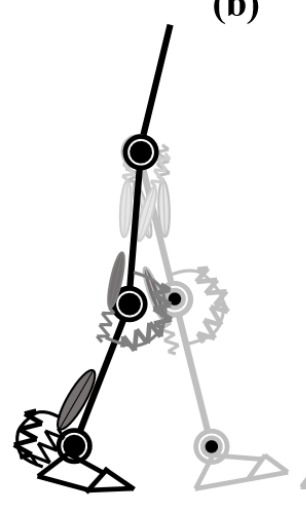

(d)

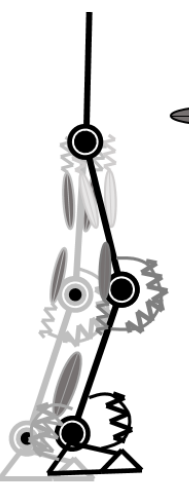

(e)

Figure 3-8. (a) Ankle-Impedance Evolution During Gait. (b-c-d) Ankle-Leg Model to Fulfill Ankle Requirements. 
During the support phase, at the loading response the ankle behaves as an elastic element. In Figure 3-8 (b), two elastic constants can be identified, this adjustment in the impedance is related to the adaptation of the foot to the ground and the load due to the subject weight. The energy to achieve this negative power can be stored at the tendons and this energy released at the push-off in combination with an active actuation as shown in Figure 3-8 (d). This power supplied at the joint, happens in a very short time window, which makes emulating this behavior with traditional electric motor a complicate task in powered exoskeletons.

During the swing phase, the foot needs to accommodate very quickly to avoid stumbling to the ground. This dorsi-flexion does not require high power because only the foot inertia is involved while the leg is in the air. In order to maintain sagittal balance, bidirectional motion at the ankle is required. A small MTU attached at the ankle can be used to actively control the foot dorsi-flexion (flexion of the foot approaching toes to the shank) or as shown in Figure 3-8 (d), an elastic element acting antagonist to the main MTU required for a proper push-off.

Table 3-1. Joint characteristics during gait, based on the MTU actuation.

\begin{tabular}{|c|c|c|c|c|c|}
\hline $\begin{array}{c}\text { Joint/ } \\
\text { Properties }\end{array}$ & $\begin{array}{c}\text { Active } \\
\text { Actuation }\end{array}$ & Spring Behavior & $\begin{array}{c}\text { Energy release } \\
\text { control }\end{array}$ & Free Motion & $\begin{array}{c}\text { Impulsive - High } \\
\text { Power in short } \\
\text { time }\end{array}$ \\
\hline Hip & $\begin{array}{l}\text { Constant power } \\
\text { generation } \\
\text { along gait }\end{array}$ & Cyclic Motion & -- & -- & -- \\
\hline Knee & $\begin{array}{l}\text { Assist ground } \\
\text { clearance, and } \\
\text { control swing } \\
\text { extension }\end{array}$ & $\begin{array}{l}\text { Loading Response, } \\
\text { and pre-swing } \\
\text { loading. }\end{array}$ & $\begin{array}{l}\text { Hold energy from } \\
\text { pre-swing }\end{array}$ & $\begin{array}{l}\text { Swing as free } \\
\text { pendulum }\end{array}$ & -- \\
\hline Ankle & $\begin{array}{c}\text { Assist ground } \\
\text { clearance, and } \\
\text { push-off }\end{array}$ & Loading response. & -- & -- & $\begin{array}{l}\text { Push-off at Toe } \\
\text { Off (Assist power } \\
\text { generation) }\end{array}$ \\
\hline
\end{tabular}

This chapter has reviewed the main mechanism involved in human walking, focusing in the required characteristics that a robotic exoskeleton should present in their sagittal joints to emulate at best the human joints. Based on the analysis performed a model of the actuation mechanism to be implemented in the exoskeleton can be developed. 
The knee joint presents multiple functions during the locomotion cycle. Prosthetic devices try to take advantage of the energy absorbed at pre-swing, in slow and moderate speed walks this required the control of the stored energy to be released when needed. In the other hand, the power supplied at the ankle join during push-off happens in a very short time window, which makes emulating this behavior with traditional electric motor a complicate task in powered exoskeletons. The property to deliver high power in short time is a particular requirement for the ankle joint. 


\section{Chapter 4}

\section{Compliant Actuation- Variable Stiffness Actuators (VSA)}

The research field focused in rehabilitation and gait assistance looks for inspiration in natural mechanisms involved in walking to deal with the necessity of compensating the many drawbacks that come along with conventional electric actuators and gear motors implementation; poor torque density, high stiffness, low force to weight ratio, high reflected inertia and lack of compliance (nonadaptability). There are currently many research groups working under the line of novel actuation development, aiming at developing and exploiting actuation technologies for a new generation of robotic devices that can co-exist and co-operate with people and get much closer to the human manipulation and locomotion performance than today's robots do [64]. With these in mind it seems natural that in the last decade a high number of new designs have come to live. These designs take into account the state of the art in artificial muscles, as well as a good understanding of the biomechanics and performance of the biological structures; to try to emulate the behavior of natural muscles in many cases and others evaluate their attributes to take advantage of some of them in order to improve some qualities of the actuation system. Novel joint prototypes should be conceived from the point of view where the robotic device is an integrated system of electronics, software, and mechanism, which should be able to passively (or almost passively) adjust stiffness, be safe, efficient, and compliant.

Traditional actuators are known as devices able to move to a specific position or tracking a predefined trajectory and once the final position is reached, it will remain at that position within the force limits of the devices. Compliant actuators are different from traditional stiff actuators; they allow deviations from its own equilibrium position, depending on the applied external force [22]. Within the requirements of the new service robotics, actuators compliance is a desired feature as in biological systems which permits deviations from the equilibrium point depending on the external force applied. The equilibrium position of a compliant actuator is defined as one in which the actuator generates zero forces and zero torque.

In order to improve understanding, the following definitions of some mechanical relationships are in order. Mechanical interaction between two systems can be modeled looking at the dynamic 
relation between the variables which characterize the energy exchange and interaction behavior between the two systems. Mechanical impedance is a differential dynamic relation which generates a force as a function of a displacement, in time domain. Stiffness is the differential relation between infinitesimal differences in force and position. Compliance is the inverse. Stiffness and compliance are related to elastic energy storage [61].

These novel actuators, are designed for particular applications and devices, thus the title of best device is a term not used in this field. Based on the VIACTORS consortium [64], these devices will pursue a common goal in terms of the abilities the actuators should achieve.

- Efficiency (e.g. natural gait generation and adaptation in legged locomotion applications);

- Robustness to external perturbations and unpredictable model errors (changes) of the environment, of the robot kinematics and dynamics, or of the dynamics of a human interacting with;

- Adaptability and force accuracy in the interaction with the operator, in applications in which continuous contact and accurate force exchange is necessary, such as in "handson" assistive devices, rehabilitation, exoskeletons and haptics;

- Safety to humans (and resilience to self-damage) in operations where the robot is required positional accuracy and swiftness of motion, while cooperating, physically interacting or even possibly colliding with the humans and their environment, such as e.g. in collaborative robotics.

Among the new actuator models, there are several works and classifications according to different features. Partially based on the work in [61] the following classification of the devices that stand out for their adaptability features is given:

\subsection{Active Impedance by control}

When an actuator mimics the impedance behavior using software control, is known as active impedance control. Based on the measured output state, a correction is calculated by the controller and set by the traditional-stiff actuator $[48,61]$. This system has an actuator, sensor, and controller that are fast enough for the application, but no energy can be stored and due to the limited bandwidth of the controller no shock can be absorbed, and the natural dynamics of walking can-not be exploited. Impedance controllers can be complex and require accurate system dynamics models. An advantage of controlled impedance is that it can adapt both the damping and stiffness online and this in a 
theoretical infinite range and with infinite speed, however in reality conventional robots in which interaction is controlled by software only, could not avoid an impact to damage the robot and possibly the human neighbor, as the controller will react with some delay [64].

ALEX exoskeleton [6], and the latest versions of the X1-IHMC [29, 40] device, are some of the current devices implementing this kind of actuation to improve the user-robot interaction.

\subsection{Inherent Fixed-Compliance}

Contrarily to the software-based approach to applying compliance to the joints, a different approach is based on providing mechanical compliance to the actuators. These actuators typically present an elastic element embedded into the system, intending to compensate for the disadvantages of traditional-stiff actuators or with active impedance by software when implemented in locomotion tasks or human-robot interaction. In the look for achieving energy storing, shocks absorption, and adaptability several actuators with inherent fixed-compliance have been designed, and some relevant realizations are presented below.

\subsubsection{Series Elastic Actuator (SEA)}

The SEA is basically a traditional actuator with an elastic element placed in series between the load and the stiff actuator as shown in Figure 4-1. The SEA continuously measures the force by measuring the deflection of the elastic element [44] and feeds back the output force. Given the deformation and using Hooke's law it is possible to determine the force/deformation curve for closedloop control that reduces the effects of friction and inertia. Compliant actuators allow for an independent control law that largely approximates to the concept of an ideal force-controlled actuator, outputting exactly the commanded force independent of load movement [47]. Some of the main features of this type of actuators are:

- Low impedance and friction thereby achieving high quality in the force control.

- Shock tolerance, impact resistance and backdriveability, even in hydraulic systems.

- The stability of force control is improved, even in intermittent contact with hard surfaces. The chatter is eliminated since a relatively large spring deflection is necessary to exert a small force.

- Energy can be stored and released by the elastic element, potentially improving the efficiency in harmonic applications [68]. 
- SEAs behave like a spring at high frequencies.

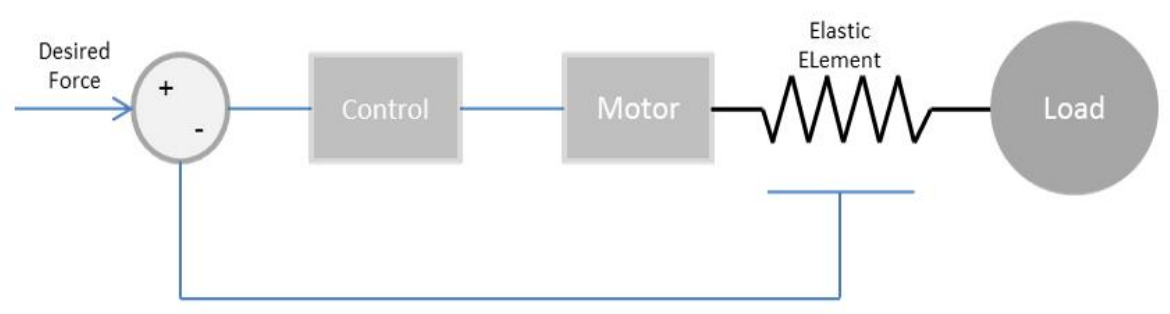

(a)

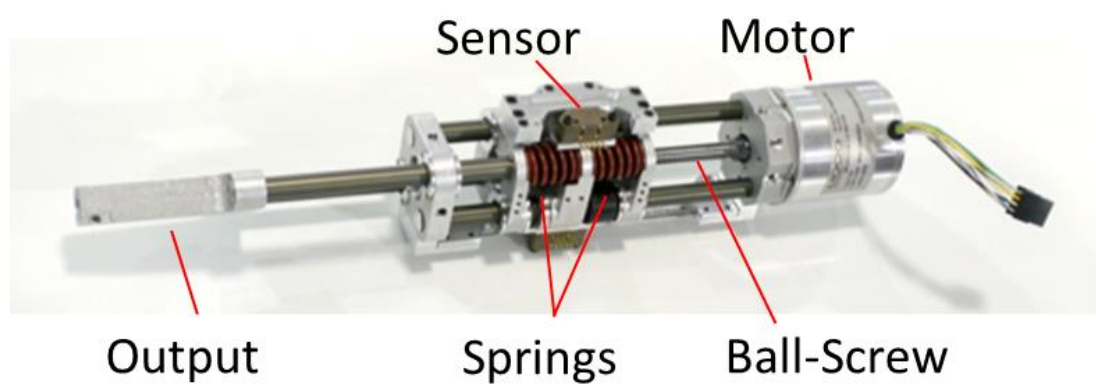

(b)

Figure 4-1 . a) Schematic Diagram of Series Elastic Actuators [44] b) Series Elastic Actuator.

Because of its muscle-like properties, low impedance, and high force fidelity, SEA stands as a good actuator to be implemented in legged robots and exoskeletons applications, and has led to the development of different actuators based on the same principle.

\subsubsection{Rotary Series Elastic Actuators (RSEA)}

These actuation systems come as an adaptation of typical SEA to be implemented in rotary actuators. Similar to the SEA concept, the stator of a rotary actuator is connected through a steel cable to a couple of linear die springs. The rotational motion is transformed to linear motion by the cables. The force transmitted to the springs is related to the moment exerted at the output of the motor gearbox allowing for force control of the system. It is characterized by low impedance and high fidelity force control and position [40].

In Figure 4-2 a conceptual schematic diagram of RSEA can be seen. When a torque is exerted at the joint a force is transmitted through the cable and applied in one of the springs, by measuring the displacement of the spring or the rotation of the gearbox and given the mechanical properties of the actuator elements, a torque can be computed with the appropriate mathematical relationships. 
RSEA systems exhibit the main advantages of traditional SEAs: low impedance and high force and position fidelity. This configuration has been successfully implemented in some exoskeleton devices and robotic legs like the compliant robotic quadruped ScarLETH [27], [26], the rehabilitation Exoskeleton (LOPES) [63], [58] and the earliest version of the IHMC Mobility Assist Exoskeleton [40].

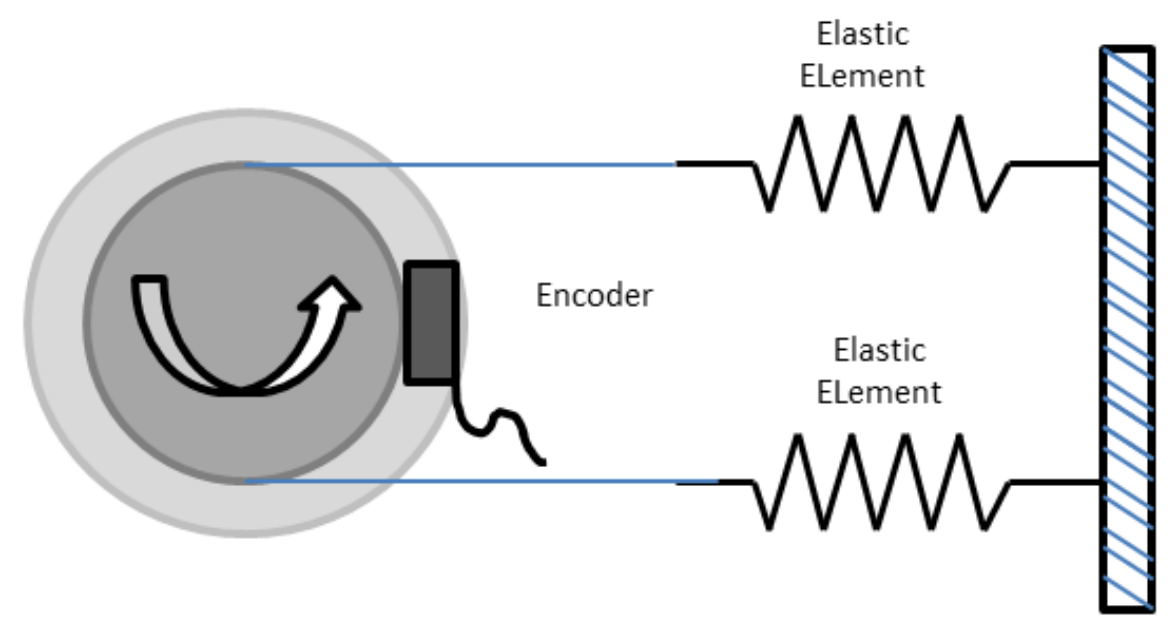

Figure 4-2. Rotary Series Elastic Actuator Schematics.

\subsubsection{Compact-Series Elastic Actuator with levels of compliance}

One of the main disadvantages with traditional SEAs is the necessity of a fixed spring for all possible conditions that the actuator should face during its operation life. The range of compliance that is required depends on the application, as is the torque requirement of the actuator [22]. In a SEA, a soft spring produces high fidelity of force control and low output impedance, but also limits the force range and the force control bandwidth at high force range. On the other hand, a stiff spring increases large force bandwidth, but reduces force fidelity. In [72] a novel adaptation of traditional SEAs is presented, in a compact design with a torsional spring placed after the motor and before the ball screw where the traditional linear springs are connected. The aim of this actuator is to improve the performance of traditional SEAs by using soft linear springs for low forces and the stiffer rotational spring for high force, increasing the force bandwidth. The actuator presents a compact arrangement 
and is intended to be implemented in wearable exoskeletons applications [45], as can be seen in Figure 4-3.

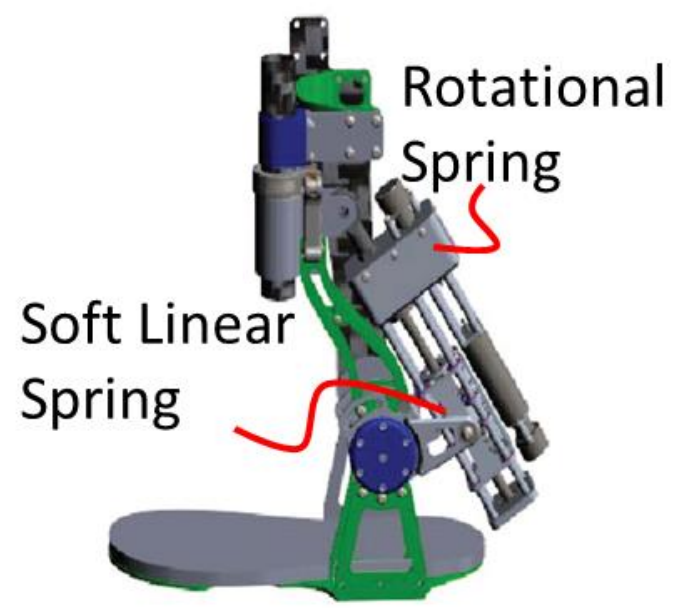

Figure 4-3. Powered Ankle with Compact Series Elastic Actuator.

\subsection{Inherent Adjustable-Compliance}

Conventional motor-gearbox designs present large energy losses due to inelastic collisions with the ground surface. Furthermore, by making use of compliant actuators it is necessary to choose a spring stiffness that in series to the ground stiffness minimizes the power consumption. As the stiffness is dependable of the weight and variable for different grounds in which we can be operating, it is advisable to use actuators with controllable compliance. Likewise, dynamic control of the stiffness is crucial in humans to adapt to changes in the environment or the requirements of the tasks performed [38], moreover, as analyzed in Chapter 3, during locomotion, joints continuously vary their stiffness along the phases of the gait. Based on their configurations or techniques through which the compliance can be adjusted VSA (also known as Variable impedance actuators, soft actuators, and adjustable compliant actuators) can be classified as follows:

\subsubsection{Antagonistic-Controlled Stiffness}

The natural muscle can only pull and not push, this is one reason why an antagonistic configuration is necessary in biological systems; one of the best known examples is the set biceps and triceps in the human arm. By contracting the biceps and the triceps relax, arm flexion occurs. When the biceps relaxes and the triceps contracts, the arm extends, in addition by contracting both muscles 
the elbow stiffness increases and when they both relax becomes very compliant and the arm hangs freely [22]. The concepts inspired by this type of configuration is found in numerous mechanical designs.

\subsubsection{Migliore's Biological Inspired joint}

In the Georgia Institute of Technology a biological inspired joint was developed [38], the design uses a basic antagonistic setup with coupled elastic devices that provide the desired quadratic force-length relationship required for stiffness adjustment in this setup configuration. Because of the lack of commercial non-linear spring availability, this joint presents a cleaver device composed for common linear springs with coupled bearings that in presence of an external force are driven through a nonlinear profile, as can be seen in Figure 4-4, producing a non-linear elasticity accordingly to the geometric profile. When both servo motors rotate in the same direction, the equilibrium position of the joint is changed, whereas by rotating in opposite directions, the stiffness of the joint is adjusted.

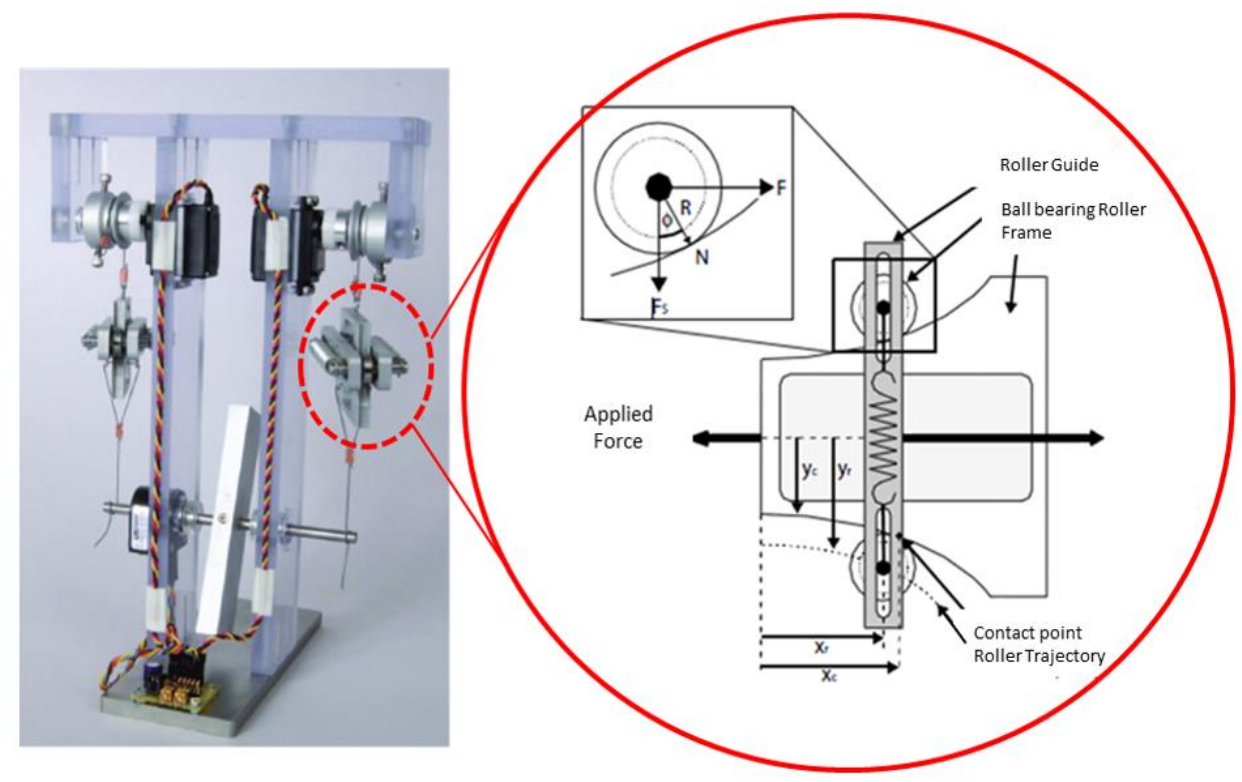

Figure 4-4. Migliore's Biological Inspired Joint and Quadratic Spring Device.

The primary concept of the quadratic elasticity device is that when stretch of length $l$ is applied to it, the springs are stretched as a non-linear function of $l$ such that the output force of the device is a quadratic function of $l$ [37]. The prototype tests reach $+40 \mathrm{mNm}$ to deflections of $60^{\circ}$, and a linear range of stiffness variation of 0.2 to $0.8 \mathrm{mNm} / \mathrm{deg}$. 


\subsubsection{Variable Stiffness Actuator (VSA)}

Tonietti et al. [56] presented a variable stiffness actuator (VSA-I) prototype, developed at the University of Pisa, composed of three pulleys over which a toothed timing belt is placed. Two pulleys are controlled each by a servomotor, while the remainder is connected to an arm, as shown in Figure 4-5. On the belt three tensioning mechanisms are placed, the two closest to the pulley mechanism connected to the arm form the nonlinear spring, and the remaining one is responsible for keeping the belt tight against the other pulleys, see Figure 4-5 (b). To achieve variation of the arm stiffness, the pulleys with servos must rotate 'counterclockwise', resulting in a compression of the springs while the spring mechanism associated with the tensioning elongates to maintain belt tension and tight to the pulleys. Likewise, when the two servos rotate in the same direction, the stiffness does not vary but the equilibrium position changes.

In [52] the evolution of the previous design, trying to increase the allowable torque and the addition of a robotic arm system is presented. The VSA-II makes use of a 4-bar mechanism and a linear spring, presented in Figure 4-6. The well-known 4-bar mechanism can be suitably designed to have desired transmission ratio between input and output they employ a simple linear spring on the input, so the relationship between deflection and torque on the output shaft can be made non-linear.

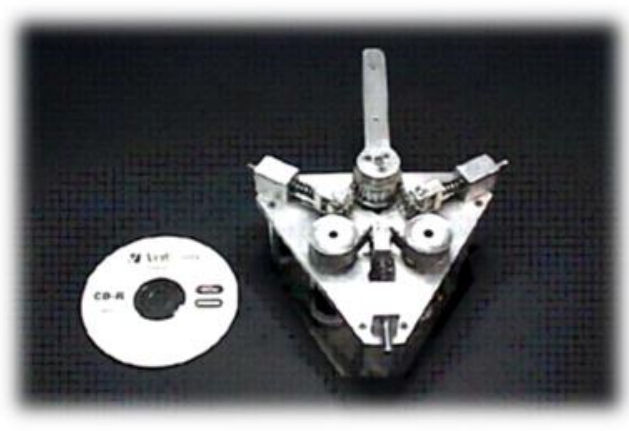

(a)

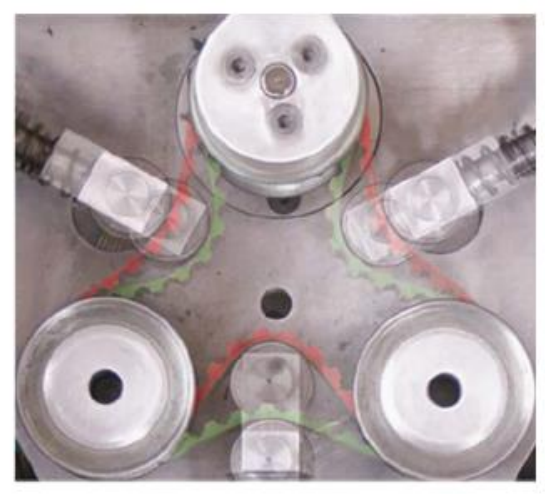

(b)

Figure 4-5. VSA-I. a) VSA Prototype b) Tensioning Mechanism. 
The $\overline{O A}$ link, with a motor in $\mathrm{O}$ commands the angular variation $\theta$, the link $\overline{B C}$ is connected to a pre-loaded torsional linear-spring and $\beta$ is the transmitted angle to $\mathrm{A}$, despite the bar linkage mechanism it is possible to achieve a non-linear relationship between input and output angles. The presented mechanism weighs a total of $345 \mathrm{gr}$ and achieves up to $200 \mathrm{mNm}$ for an approx. deflection of $2^{\circ}$ and the stiffness range goes from $8 \mathrm{Nm} / \mathrm{rad}$ to almost zero.

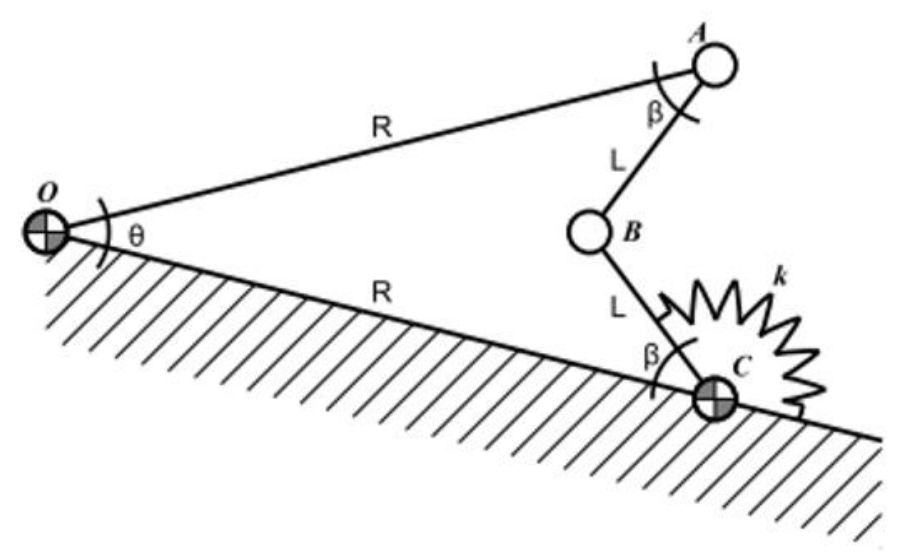

Figure 4-6. 4 Linkage Bar Mechanism of the VSA-II.

\subsubsection{AMASC}

Hurst et al. [25] design an actuator that physically achieves stiffness variation, the Actuator with Mechanically Adjustable Series Compliance (AMASC), is another device based on the antagonistic configuration and was developed at Carnegie Mellon University. It is a complex mechanism with the main advantage that only one actuator is used to control the stiffness or equilibrium position. Each actuator having a specific function allows the use of different types of motors to optimize the weight of the entire system. AMASC was conceived as a biped leg actuator, aims to achieve stiffness adjustments during operation for efficient running gaits and adaptability to different terrains. It presents a pulleys mechanism with a certain configuration that allows a linear and a non-linear behavior of the spring elements in the actuator as desired for the task performing. In Figure 4-7 a schematic drawing of the pulleys system is shown. The structure is composed of aluminum rods and a system of cables, pulleys and fiberglass which play the role of the large springs. The prototype weighs $4 \mathrm{~kg}$ and allows physical variation of the stiffness, remote positioning of the engines and no significant limitations when bandwidth is adjusted properly. 


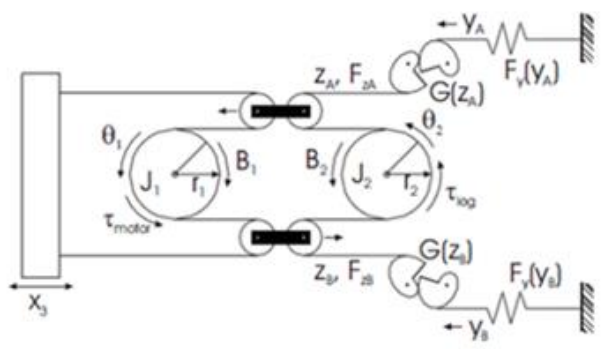

(a)

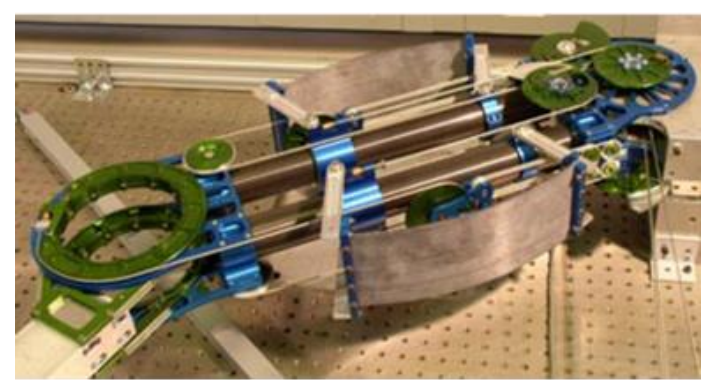

(b)

Figure 4-7. 4 AMASC-a) Schematic Diagram of Pulleys Mechanism and b) $1^{\text {st }}$ Leg Prototype.

The ideas of the AMASC were incorporated in a full bipedal robot, the BiMASC (Biped with Mechanically Adjustable Series Compliance), this new device presents an Electric Cable Differential (ECD) leg designed by Dr. Hurst for running, walking, jumping, hopping, and generally behaving in a highly dynamic manner. The large fiberglass springs are used for storing the energy of a running gait [41]. In this design through the ECD the motors are connected to the leg joints using steel cable wrapped around aluminum pulleys, and there are several mechanical differentials to implement the desired relationship between the electric motors, fiberglass springs, and leg joints.

\subsubsection{Quasi-antagonistic Joint (QA-Joint)}

For the Quasi-Antagonistic Joint (QA-Joint) [13], developed at the German Institute of Robotics and Mechatronics (DLR), one motor adjusts the link side position, while the second motor operates the stiffness adjustment. This is a partially decoupled system since when the stiffness is changed, the equilibrium position must be adjusted by the link side motor. In Figure 4-8 (a) $\sigma$ and $\theta_{1}$ are the actuation variables, the elastic force transmitted depends $\varphi+\frac{\sigma}{2}$ and consequently a large stiffness variation can be achieved. The variables $\theta_{1}, \theta_{2}$ and $\sigma$ or all together can be acted, since the transmission elements can move independently from each other. Figure 4-8 illustrates the operation scheme where 
$\theta_{1}$ and $\theta_{2}$ are absolute positions of the curves and $\theta^{*}$ the equilibrium position. The displacement between the curves determines $\sigma$

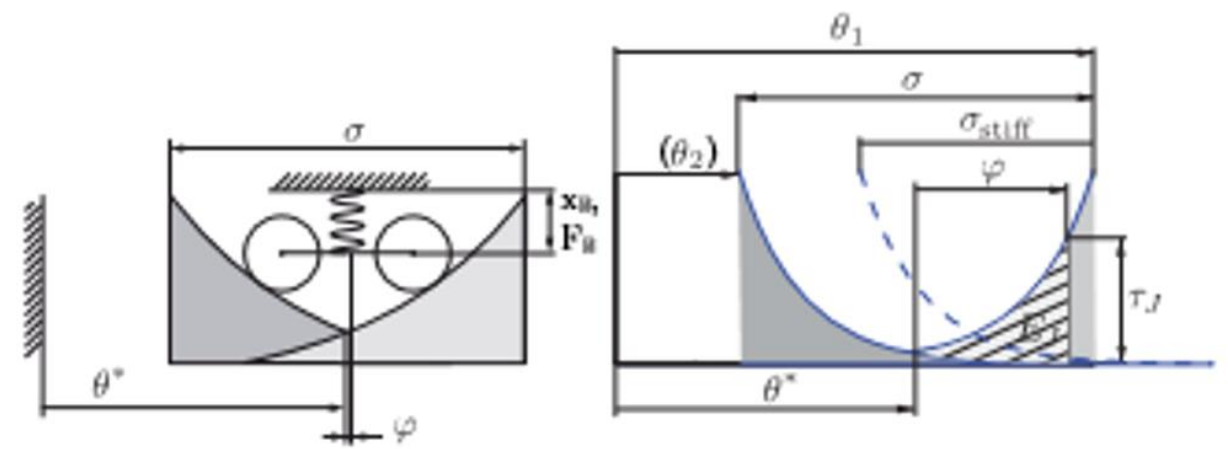

(a)
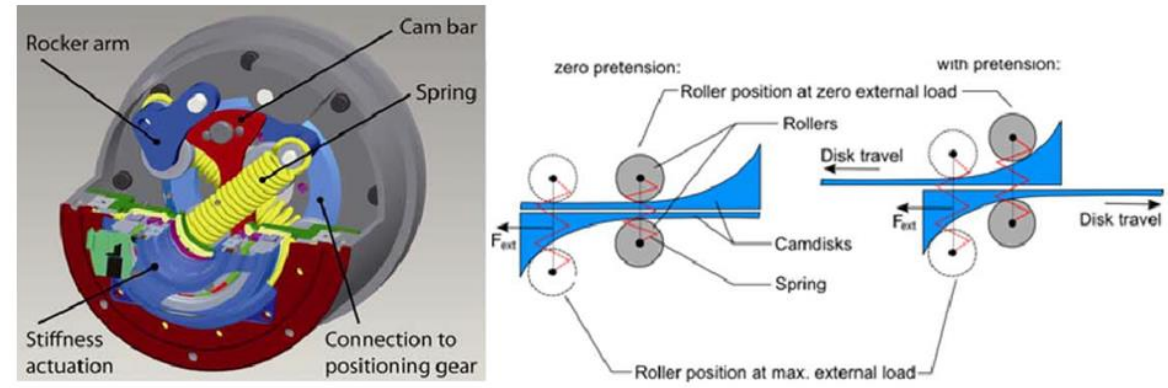

(b)

Figure 4-8. Quasi-Antagonistic Joint. a) Transmission Variable Design. b) QA-Joint and Principle of Operation.

The compliance system consists of two mutually opposed elastic elements supported so as to allow a variable range of elastic mobility. Figure 4-8 (b) shows how the cam discs are placed with or with-out pretension. In the presence of external forces a rotational displacement occurs that is restricted by the set of springs. From tests evaluations, they report joint torques up to $\pm 40 \mathrm{Nm}$ and stiffness range from $20 \frac{\mathrm{Nm}}{\mathrm{rad}}$ to $550 \frac{\mathrm{Nm}}{\mathrm{rad}}$ achievable in $0,12 \mathrm{~s}$ in a joint system of $1,2 \mathrm{~kg}$.

\subsubsection{Preload Adjustment of Single Spring}

Different to antagonistic setups, this group of mechanisms achieve the stiffness adjustment by mechanically varying the points where the compliant elements are attached to the structure. In the last few years a high number of new designs have been developed for different research groups that fit into this category. In 2009 the European project entitled VIACTORS started, this project was composed of six European partners and was addressed to the development and use of safe, energy-efficient, and highly dynamic variable-impedance actuation systems which will permit the embodiment of natural characteristics, found in biological systems, into a new generation of mechatronic systems. Many of 
the following systems come as a result of this project [64]. The main feature of this subclass is the use of a nonlinear connector between the output link and the spring element hence only one linear spring is required. The stiffness adjustment is however still performed by changing the preload on this single spring [61].

\subsubsection{MACCEPA}

The Mechanically Adjustable Compliance and Controllable Equilibrium Position Actuator is a design from Vrije Universiteit Brussel, in which the compliance can be controlled separately from the equilibrium position. Each of these parameters is set by a position controlled servo motor [60]. The torque is a linear function of the compliance and of the angle between equilibrium position and actual position. The working principle of this actuator is shown in Figure 4-9 (a); three bodies pivoting around one rotation axis that can be seen as a knee joint, where a spring is attached between a fixed point on the lever arm "c" and a cable running around a fixed point " $\mathrm{b}$ " on the right body to a pretension mechanism. The angle $\varphi$ between the lever arm and the left body is set by a classical actuator. When $\alpha$, the angle between the lever arm and the right body, is different from zero, the force due to the elongation of the spring will generate a torque that tends to align the right body with the lever arm. The equilibrium position is determined by a motor that varies the angle $\varphi$. A second actuator, which pulls on the cable connected to the spring, will set the pretension of the spring. This pretension will
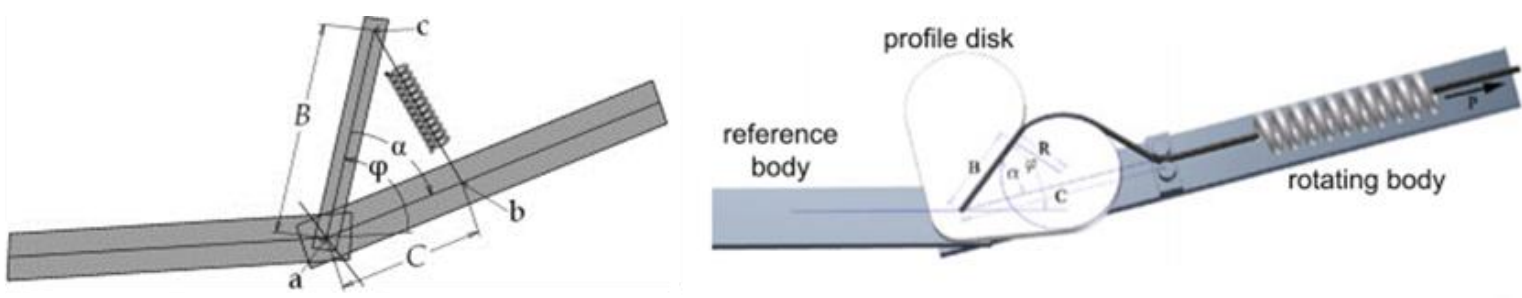

a)

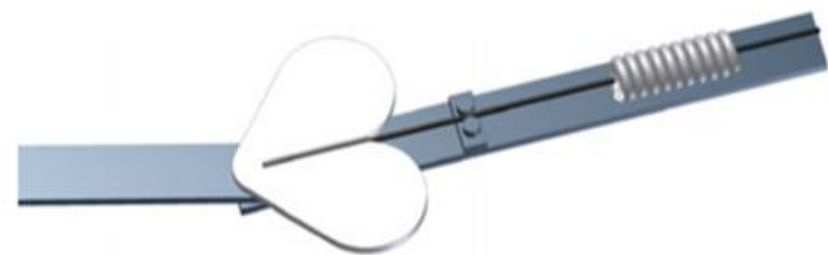

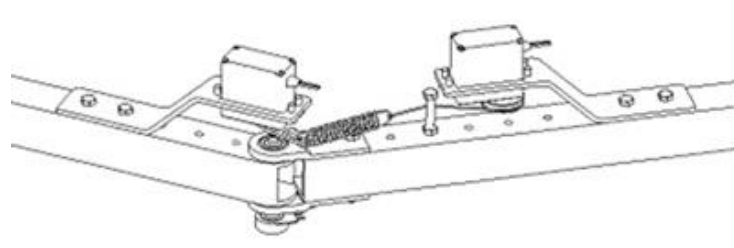

b)

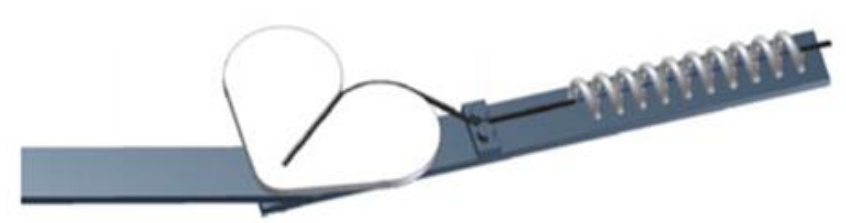

c)

Figure 4-9. a) MACCEPA Basic Configuration. b) MACCEPA Prototype. c) MACCEPA 2.0 Schematic Drawing. 
vary the torque for a certain angle $\alpha$, thus controlling the spring constant of an equivalent torsion spring.

The MACCEPA 2.0 is the follow-up version where the torque-angle curve and consequently the stiffness-angle curve can be modified by choosing an appropriate shape of a profile disk, which replaces the lever arm of the original design, see Figure 4-9 (c).

The MACCEPA 2.0 system has been incorporated in the knee joint in the ALTACRO (stands for Automated Locomotion Training using an Actuated Compliant Robotic Orthosis) project, and aims the development and clinical testing of a step rehabilitation robot powered by compliant actuators to improve the quality of step rehabilitation therapy both for patients and therapists and increasing the availability of automated step rehabilitation training. The tests performed [10] have shown that the implemented joint achieved a peak torque up to $70 \mathrm{Nm}$ and a continuous torque of $50 \mathrm{Nm}$. The stiffness can be adjusted in 2,6s from $5 \frac{\mathrm{Nm}}{\mathrm{rad}}$ to $110 \frac{\mathrm{Nm}}{\mathrm{rad}}$, and the weight of the overall joint is of $2,4 \mathrm{~kg}$.

\subsubsection{Variable Stiffness Joint (VS-Joint)}

The Variable Stiffness Actuator (VS-Joint) [1] from the DLR, [70] contains two motors of different size, the high power motor changes the link position while the joint stiffness is adjusted by a much smaller and lighter motor. In Figure 4-10, the figures on the right (b) show a schematic of the working principle. A compliant link deflection results in a displacement of the cam disk and is counterbalanced by the roller pressed on it in axial direction by a spring. This generates a centering force resulting in the output torque of the link. To change the stiffness preset, the smaller motor moves the spring base axially (fixed to the gearbox output) to the cam disk and thus varies the spring force.

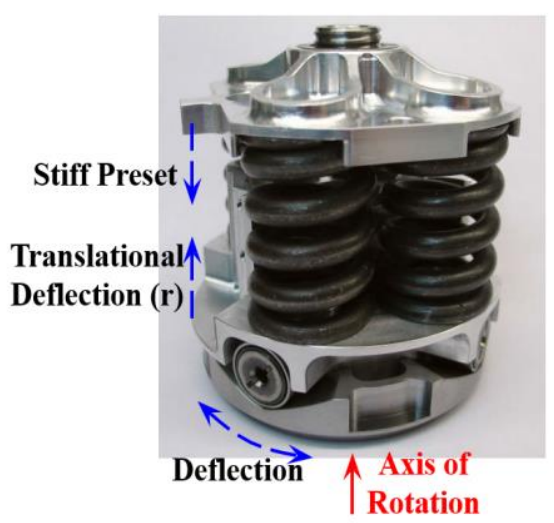

(a)

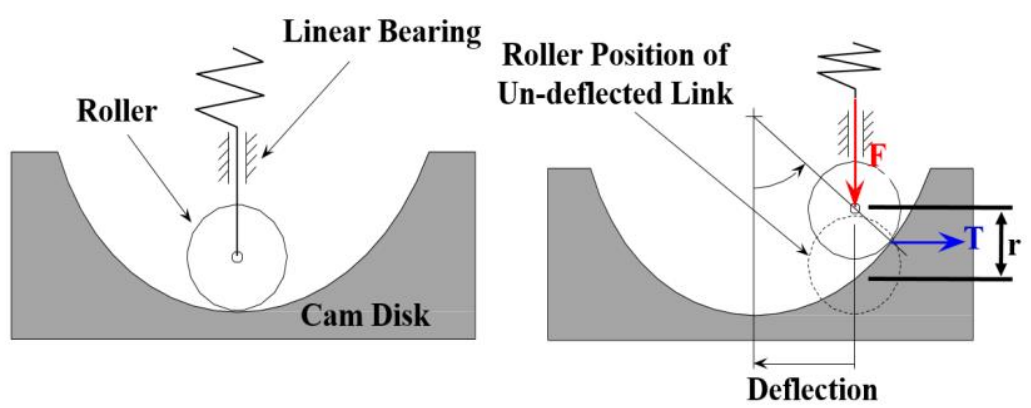

(b)

Figure 4-10. a) Mechanism of the VS-Joint. b) Working Principle of the VS-Joint. 
From the published results after repeated evaluations the VS Joint can achieve torques up to $\pm 180 \mathrm{Nm}$ with a range of stiffness between $0 \frac{\mathrm{Nm}}{\mathrm{rad}}$ to $315 \frac{\mathrm{Nm}}{\mathrm{rad}}$ with a stiffness adjusting time from $3 \%$ to $97 \%$ stiffness in 0,2 s for a system of about $2 \mathrm{~kg}$ weight.

\subsubsection{Changing Transmission between Load and Spring-Lever Length.}

Focused on the Lever Length subdivision of this group of actuators, the stiffness is adapted by changing the transmission ratio between the output link and the spring element. In contrast to the previous category this design does not preload the spring, and little to no energy is required to change the stiffness since the force on the spring is orthogonal to the spring displacement. Friction, and little projection of the actuator holding torque has to be overcome. However this energy is significantly lower than the required in the preload adjustment.

The Italian Institute of Technology (IIT) has come up in the last few years with a high number of new cleaver designs and designs evolutions of variable impedance actuators from this category.

\subsubsection{CompAct ${ }^{\mathrm{TM}}$-VSA}

A compact actuator that varies its compliance is shown in Figure 4-11, the CompAct-VSA [57] makes use of a cam shaped lever arm $\mathrm{C}$, with a variable pivot axis $\mathrm{P}$ that can move along $\mathrm{B}$ actuated by a rack and pinion transmission system $\mathrm{F}$ and $\mathrm{G}$. When a torque is exerted the springs $\mathrm{H}$ are contracted by the cams $\mathrm{E}$ transmitting the force to the joint. By adjusting the pivot axis $\mathrm{P}$ position, the stiffness in the system can be adjusted. The required power from the stiffness motor is lower than the required in systems where the stiffness is regulated by comprising or elongating the stiffness [65], because the force is not acting in the direction of the spring action.

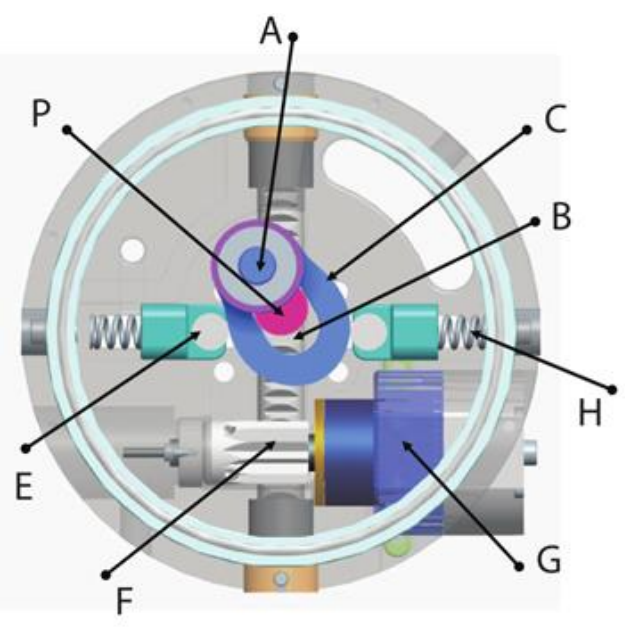

Figure 4-11. CompAct-VSA Mechanism. 


\subsubsection{AwAS I and II}

The Actuator with Adjustable Stiffness (AwAS-I) [31] is based on a compliant lever which rotates around a fixed pivot point as can be seen in Figure 4-12 (a). Its compliance is due to two springs attached antagonistically on the lever at a variable distance. The stiffness adjustment is done through moving the springs along the lever and changing the lever arm. A Motor controls the position and is connected to the intermediate link while a second motor (smaller) is assembled on the intermediate link and is attached to a ball-screw which varies the pivot distance from the joint axis (lever arm length). Springs are connected between the ball screw and output link and move together with the ballnut.

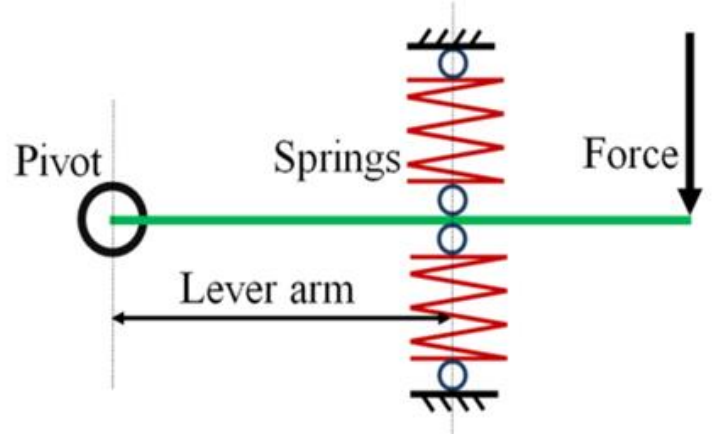

(a)

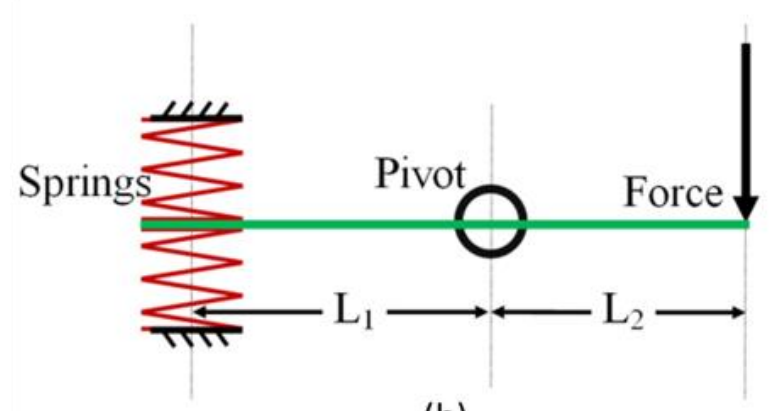

(b)

Figure 4-12. a) AwAS I Mechanical Principle and b) AwAS II Mechanical Principle.

The AWAS II [30] is the evolution of the previous design, in this version springs are kept fixed, and replaced for rotational ones, but the pivot point is variable, see Figure 4-12 (b). The stiffness of the lever depends on the ratio L1/L2 and can be adjusted from zero (pivot reaches springs position) to highly rigid. A torque up to $80 \mathrm{Nm}$ is achieved for both designs and the system weight reduced from $1,8 \mathrm{~kg}$ to almost $1,1 \mathrm{~kg}$ in the AwAS-II, the stiffness adjusting time remains in $0,8 \mathrm{~s}$ from $3 \%$ to $97 \%$ of its stiffness range. 
In Figure 4-13 the CAD prototypes of both systems can be seen, the regulation system presents advantages in comparison with other devices, such as the DLR joint, where a more powerful motor is required because the compression force required for stiffness adjustment is performed in the same direction that the spring forces act.

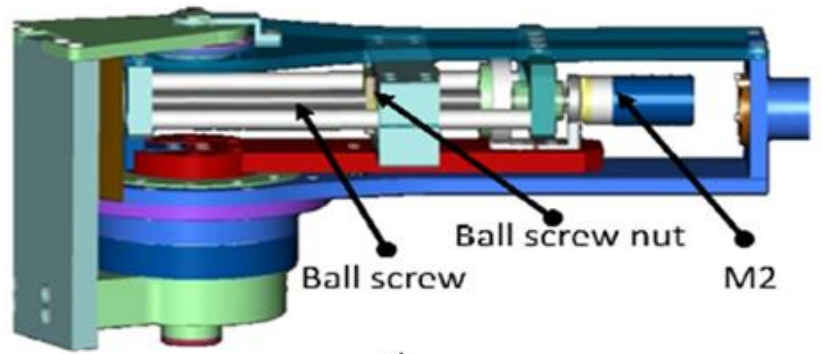

a)

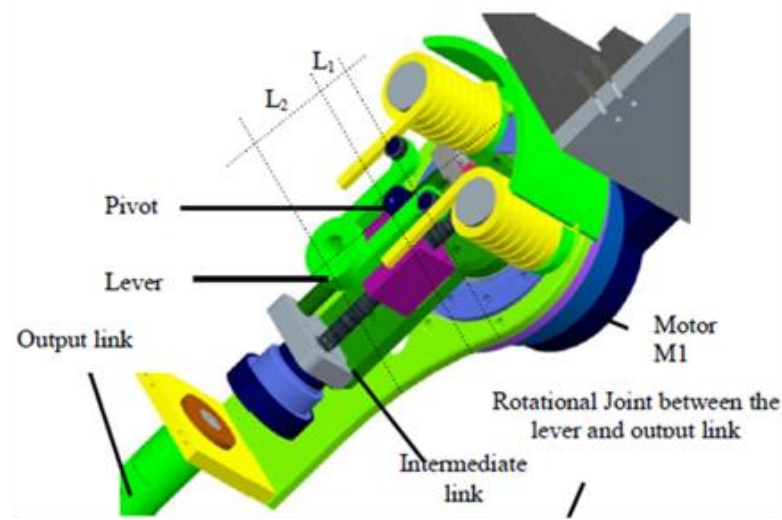

b)

Figure 4-13a) AwAS b) AwAS II CAD prototypes.

\subsubsection{Controllable Variable Impedance Actuators (VIA)}

Table 4-1 compares current joint developments, presenting some of their key characteristics, many of the presented joints show very good properties for exoskeleton applications; future evolutions of these designs should focus on size and weight reduction. From the comparison, several parameters seem relevant to take into consideration;

- Stiffness should be changed without considerable energy consumption, in order to reduce the power requirements of the system.

- Low to no energy should be required to maintain the stiffness, and stiffness should be independent from the external load. These will avoid the need of large motors or elements for stiffness adjustments. 
- Large Range of stiffness is desired to improve versatility and allow the application of the system to different users, and applications.

- No inertia should be added to the system output due to the stiffness adjustment mechanism.

- Maximum capacity of the energy storage should be accessible, because this could translate in energy efficiency, and less power required by the main actuator to produce a certain torque.

Compliance has an important role in enhancing the capabilities of robots to deal with unknown environments. Different variable stiffness actuators have been developed so far, answering the need of different applications. Some of the designs presented in Table 4-1 are conceived to be used in a specific joint, knee and shoulder joints integrate some of the same key elements, just with different torque requirements. Considering the previous four characteristics that the authors highlight as part of the key elements for exploiting natural dynamics, the designs of the VS-Joint and QA-Joint from the German Aerospace Center (DLR) and those from the IIT seem to collect most of the desired elements in terms of stiffness variation in range and required time for adjustment. Size is considered excessive for wearable devices, although compact; the parallel arrangement of the components turns in a bulky joint. Although, none of them can be used for general purposes. Certain characteristics should be adopted or improved in future designs. The compression mechanism has to oppose directly the reaction force of the actuator, while the Pivot Displacement mechanism mostly present in the Italian Institute of Technology (IIT) designs, just oppose a projected part of that force, according to the deflection in the joint, therefore the required energy for adjusting stiffness is expected to be lower than when stiffness is adjusted by compression in the same direction that the elastic element acts. The lower force required to be overcome, the lower the extra energy required for the extra actuator. 
Table 4-1. Actuators Comparison

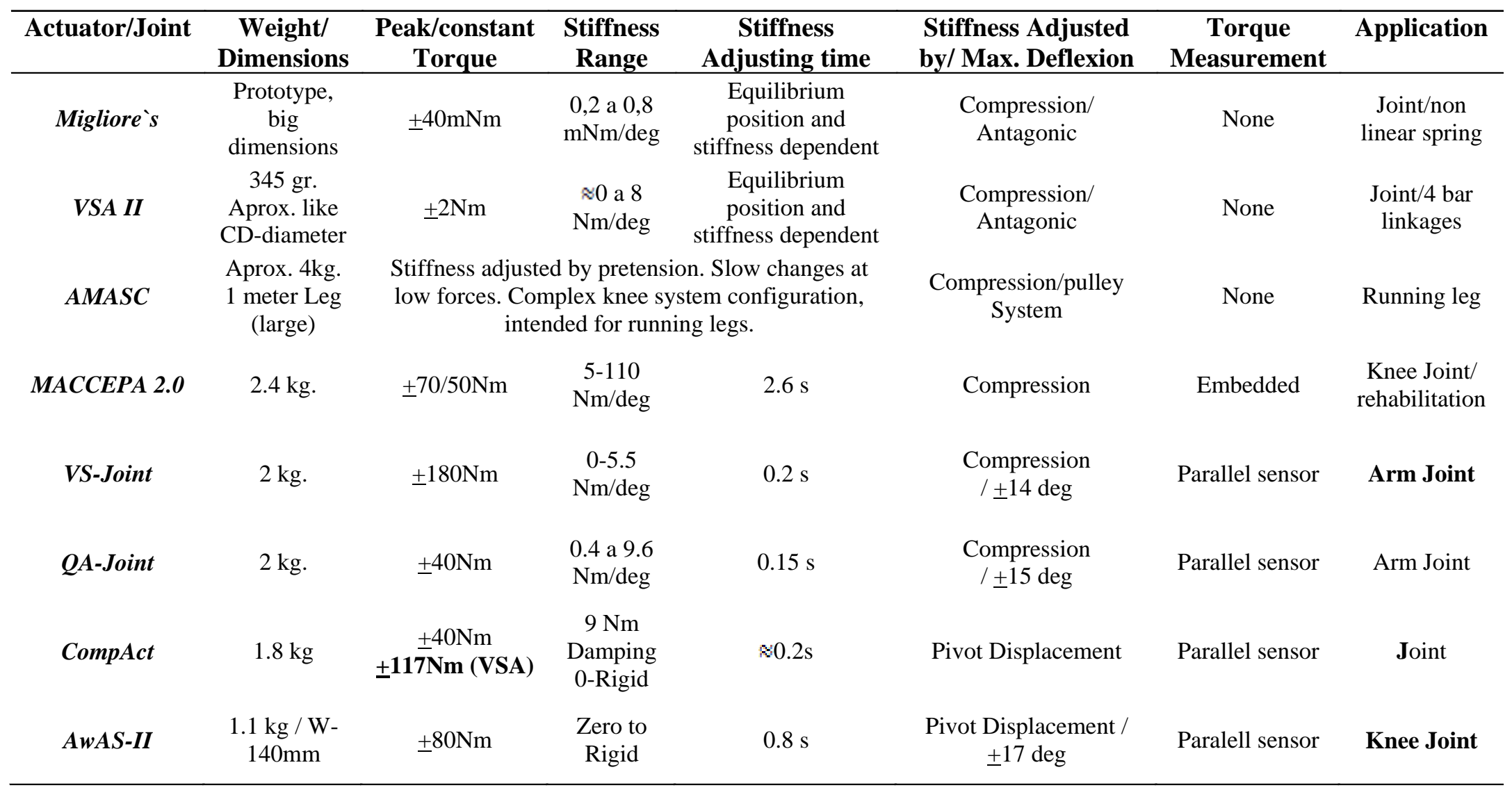




\section{Chapter 5}

\section{Actuator of Adjustable Rigidity with Embedded Sensor-ARES}

In the previous Chapter a wide range of compliant actuators were presented, these devices report many properties that match with some of the main requirements for different joints in robotic exoskeletons, but size, mechanism-complexity and requiring extra elements for force/torque measurements are some of the few drawbacks that this $\mathrm{PhD}$ thesis work tries to solve with a new design that ultimately will be implemented in the ATLAS lower limb exoskeleton.

This chapter is divided into three main sections that address an overview of the requirements that should be taken into consideration for designing novel compliant actuators, details of the mechanism that conform the compliant joint developed based on the expected joint requirements, and the assessment of the actuator's prototype of Adjustable Rigidity with Embedded Sensor-ARES [9].

\subsection{Compliant Joint Requirements}

Some of the capabilities and characteristics, particularly focusing on the knee joint, considered in the design process of the novel joint actuator that would allow exploitation of the natural dynamics of the leg during gait and adopting the joint behavior desired and analyzed in Chapter 3, are briefly discussed below.

\subsubsection{Size and Dimensions}

The user that would wear the device has to be able to handle it. For this, small sizes and reduced weight are required for exoskeleton applications and also because aesthetic of the device is important to the user. Reduced size is intended not to bother in regular activities of the user and by having lower dimensions the associated inertia that the actuator has to manage should decrease. Series arrangements are preferred instead of parallel configurations, to avoid excessive length in the joint system, thus smaller dimensions can be achieved. 
From observation and experience during the development and evaluation of the first ATLAS prototype, we have come to the conclusion that over 90mm of extra length from the outside leg, the actuator system becomes an obstacle when the user tries to sit, uses a walker or passes through a door.

\subsubsection{Stiffness Range}

The range of stiffness represents the ability of the actuator to go from very stiff to the minimum stiffness in the mechanism, thus adjusting the mechanical impedance in the device, as desired in new powered orthosis. Although, as it has been said, joint stiffness should vary depending on ground stiffness, gait speed, terrain irregularities, and other factors; by analyzing the behavior of the joints during locomotion, three main states of stiffness were identified as desired,

- High stiffness for giving impulse in the toe-off, nearly rigid. For a child of $35 \mathrm{~kg}$ with a peak torque near to $20 \mathrm{Nm}, 10-20 \mathrm{Nm} / \mathrm{deg}$ should be enough to provide the required impulse.

- Medium stiffness for adaptation purpose. A trade-off between joint and ground stiffness determines the best value that the joint should adopt. Also walking speed, user weight and ground irregularities affect the determination of this value, this is why is not fixed and the ability for adjustment is desired in a complaint actuator. From the analysis of the data from CGA at the knee performed in Chapter 3, a bump at the beginning of the support phase can be observed, this reaction at the knee is due to the load response when the heel-strike occurs. The knee should adapt this 6 to 10 degrees of deflection while providing torque up to $20 \mathrm{Nm}$.

- Minimum stiffness for swing phase. During the end of the swing as was observed in Chapter 3, taking advantage of potential and kinetic energy when hip extends the knee is almost free (near to zero stiffness), and achieves full extension without the need of positive power in that joint. 
Table 5-1. Different Stiffness Ranges at the Knee Joint.

\begin{tabular}{ccc}
\hline High Stiffness & Medium Stiffness & Low Stiffness \\
& & \\
\hline Toe-Off (Impulse) & Load response and & End Swing \\
Initial-Swing & ground adaptation & (Knee extension) \\
(Knee Flexion) & & \\
& & \\
\hline
\end{tabular}

\subsubsection{Force Sensor Embedded}

By force-controlling the joint, dynamic control of the joint for adapting to changes in environmental conditions is possible. Robots and actuated systems, even more when humanmachine interaction exists, must have the capability of sensing and controlling forces in addition to knowing where they are in their work space. To maintain a reduced size and dimension, the force sensor has to be embedded in the mechanism.

From Chapter 3 the range of torque at the knee joint goes from approximately $-30 \mathrm{Nm}$ to $60 \mathrm{Nm}$ in a $70 \mathrm{~kg}$ adult at a walking speed of $6 \mathrm{~km} / \mathrm{h}$ and from $-17 \mathrm{Nm}$ to $40 \mathrm{Nm}$ for a child of around $35 \mathrm{~kg}$ at the same speed. The force sensor incorporated has to be able of working at these ranges, moreover during swing phase to take advantage of the potential and kinetic energy by incorporating a zero-force control, the sensor has to be able of detecting small torques to avoid extra energy drain in the motors.

\subsubsection{Stiffness Adjustment Speed}

High speed is required in the stiffness variation to take advantage of the elasticity and leg dynamics. The energy stored in the elastic elements is released immediately after the opposition force disappears, at the speed of recovery of the elastic element; usually high, for so a fast variation of stiffness is required to take advantage of this released energy in the total motion.

Many of the actuators evaluated in Chapter 4, introduce a second motor to regulate stiffness, this motor is considerable less powerful than the joint position controller motor. However, the small motor has to overcome the system resistance to the adjustment. Stiffness 
adjustment by compressing the elastic element requires enough power to overcome the spring opposite force.

The pivot point principle or controllable transmission ratio seems to be the best selection for stiffness regulation because the second motor does not have to overcome the elastic elements

opposite force due to operational principle of pre-compression. A high speed-linear motor is intended to adjust the stiffness in the mechanism in less than $1 \mathrm{~s}$ to aim the properties of the best designs in this matter.

\subsection{ARES Design.}

ARES is originally conceived as a knee joint for the ATLAS lower-limb active orthosis, which controls independently its equilibrium position and its compliance. Being addressed to children it makes the torque control together with maintaining reduced lateral size a main goal for the design. In this section, the components of the actuator are presented and its adjustable compliance and embedded force sensor working principles are shown and explained.

\subsubsection{Joint Mechanics}

In Figure 5-1 a CAD image of the presented actuator is shown connected to a set of links that represent the knee of the active orthosis. The actuator can be broadly divided into two main components, the stiff complex and the compliant mechanism, arranged along the structure in order to reduce the lateral size of the mechanism.

\subsubsection{Stiff Complex}

The stiff complex presents a traditional combination of stiff actuator and a gearbox. To achieve the required high size/torque during locomotion cycle and weight/torque ratios demanded in this application, flat Maxon 90W brushless DC motor in combination with Harmonic Drive unit with 100:1 reduction ratios were selected. The motor, see Figure 5-1 b), and the Harmonic Drive combination, denoted as M1, control the equilibrium position of the joint and integrate with the slotted bar, the stiff complex. Maxon Flat Motor was selected because of its reduced length and high torque, which makes it very suitable for exoskeletons and active orthosis applications. After a reduction of 100:1 ratio the nominal outputs of the stiff set can go up to $2.5 \mathrm{rad} / \mathrm{sec}$ and $30 \mathrm{Nm}$, enough to achieve the requirements during the locomotion cycle in level ground at low speed. The 
Stiff complex output, is connected to the articulated lower link. A rotary sensor connected to the joint, measures the angle position.

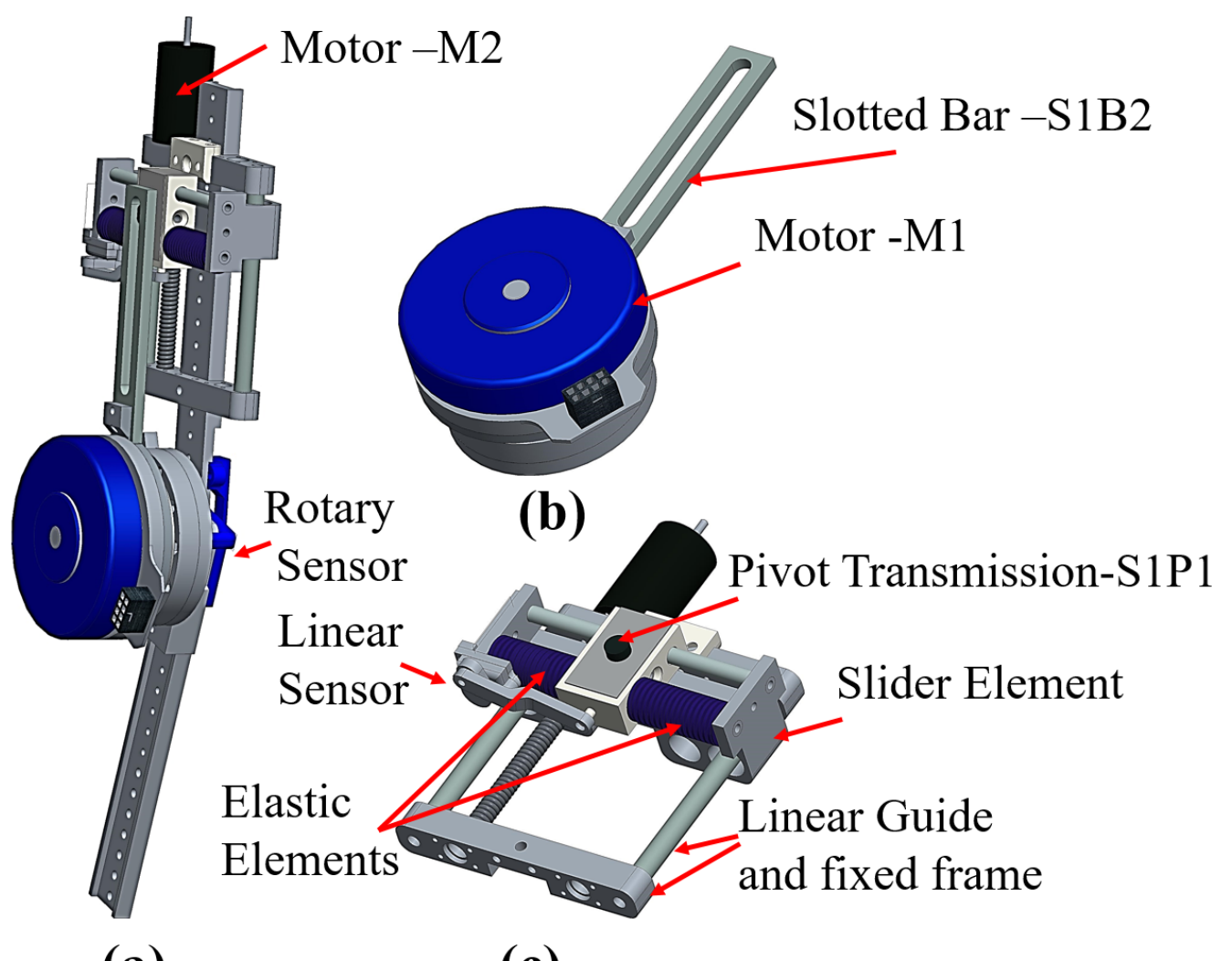

(a)

(c)

Figure 5-1. ARES Joint Mechanics. a) CAD Drawing of ARES, b) CAD of Stiff Complex, and c) CAD of Compliant Mechanism.

\subsubsection{Compliant Mechanism}

The compliance of the joint is governed by this mechanism, a set of elastic elements placed in a slider element that is connected to a spindle drive for adjusting the slider position along the linear guides of a fixed frame, see Figure 5-1 c).The set of elastic elements can be comprised by 1 or 2 pairs of elastic elements, pre-compressed a distance larger than the half of the maximum expected compression. A linear sensor implemented between the slider element and the element moving together with the pivot-S1P1, allow the measurement of the elastic elements compression. 
A Maxon AC motor, RE-25, of $20 \mathrm{~W}$ (considerably smaller that the main motor in the M1 combo) connected to the spindle, comprises $-\mathrm{M} 2$. The complex $-\mathrm{M} 2$, exert the required force for varying the slider position when it is commanded.

Both main components are coupled by the contact between the Rolling Pivot -S1P1, and a point along inner face of the slotted bar -S1B1 (see Figure 5-2). With this configuration no extra length is added in the lateral dimension, and the components can be spread along the exoskeleton's links. The linear guides in the fixed frame of the compliant mechanism support the forces transmitted by the stiff set into the compliant mechanism. The ball-screw will experience significant forces only in the axial direction, during large deflections, and no bending forces are expected. This allows the use of a lighter spindle.
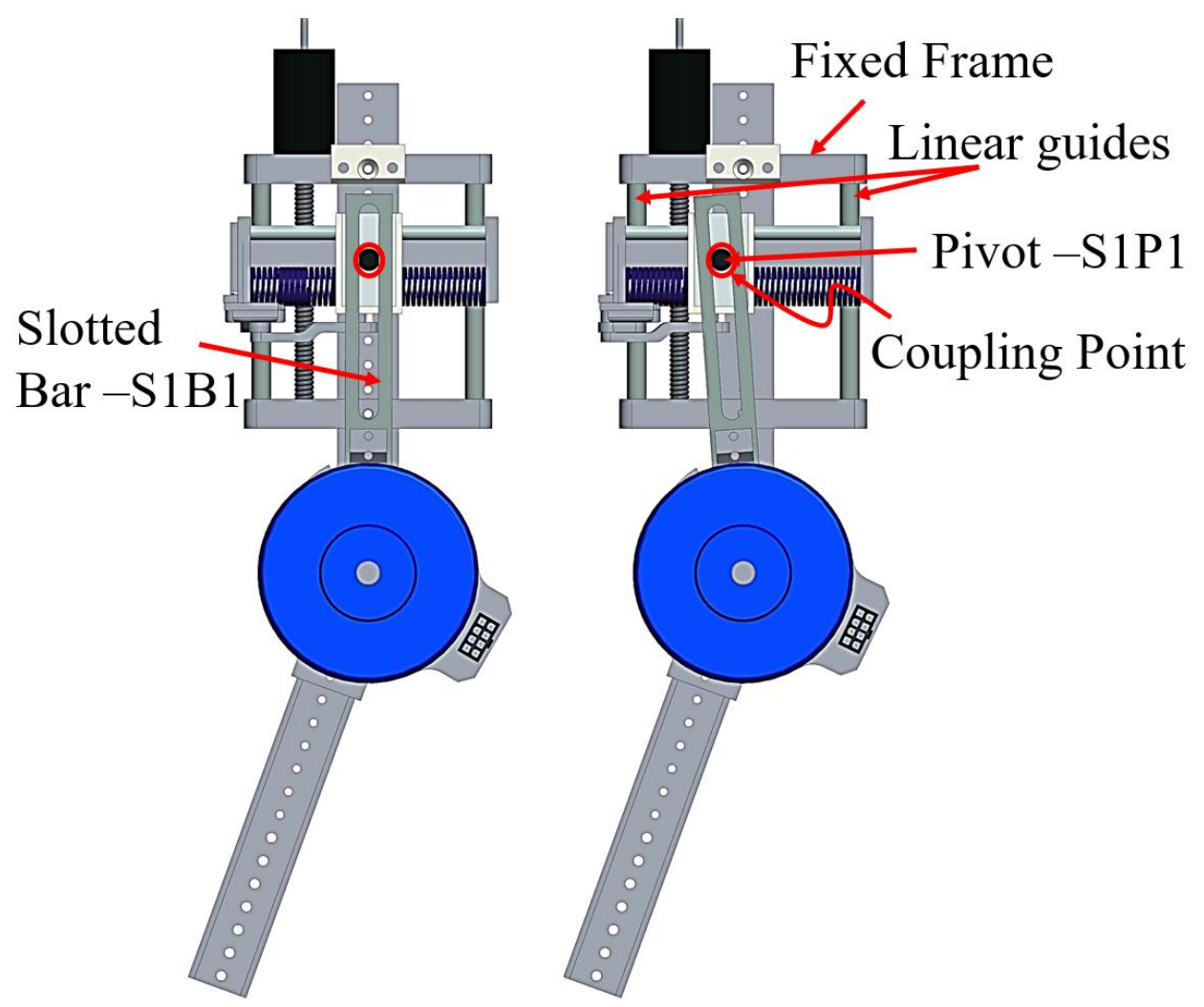

Figure 5-2. ARES Components Coupling 


\subsubsection{Working Principle}

Based on the work of the VIACTORS consortium [62], ARES can be classified as an actuator with controllable transmission ratio. When a torque is exerted at the joint, a reaction force is transmitted through the slotted bar -S1B1 to the contact with the compliant mechanism -S1P1. The force in the coupling between the rolling point -S1P1 and the bar S1B1, see Figure 5-2, will produce a compression in the elastic element, which opposes the force until the equilibrium is reached. In Figure 5-3, it can be noticed in the diagrams, that the elastic elements' compression, $\Delta \mathrm{X}$, produces a deflection $\boldsymbol{\alpha}$. The distance between the joint axis and the slider system containing the elastic elements is related with the amount of deflection experimented at the actuator system at the same external or exerted torque. The larger the distance $\boldsymbol{L}_{\mathbf{0}}$, the smaller the deflection $\boldsymbol{\alpha}$ as can be noticed in the diagram in Figure 5-3. Thus, by controlling the position of the slider system

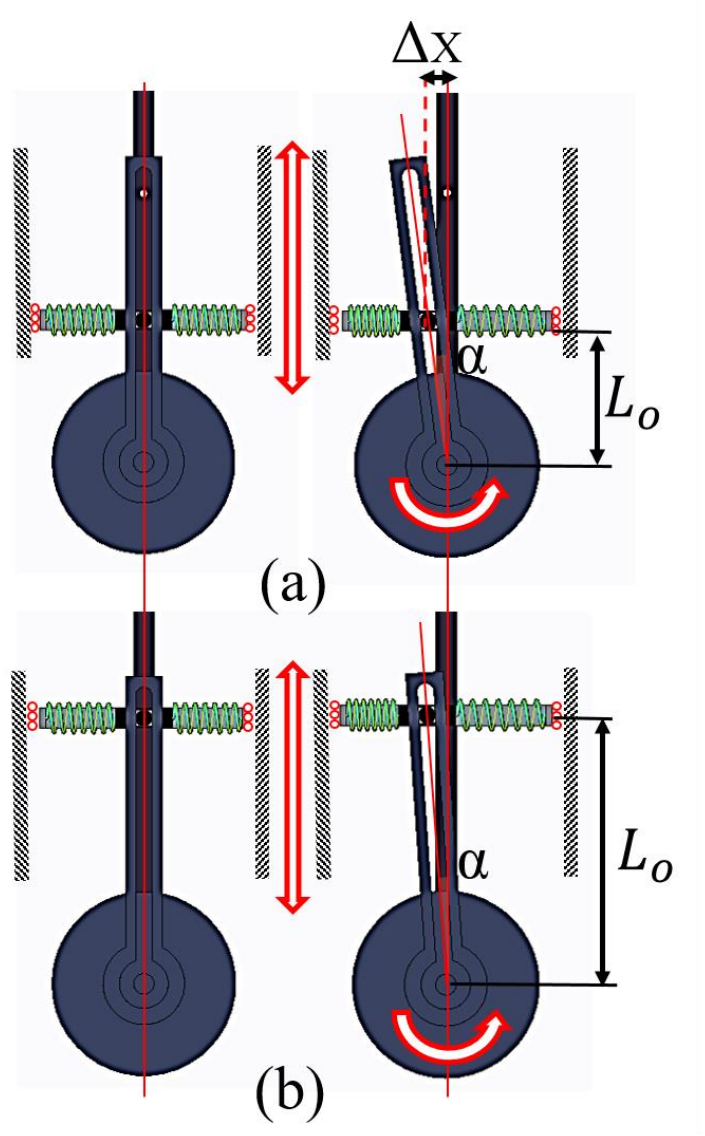

Figure 5-3. ARES Working Principle. a) Less Stiffness Configuration, b) High Stiffness Configuration. 
with the use of the motor M2 in the compliant system, a variation of the output stiffness can be achieved.

The controllable transmission ratio maintains the power necessary for the variation of the arm length low. The force produced by the motor M2 does not act directly on the elastic elements, thus just need to overcome friction and a small component of the springs reaction when trying to adjust the compliance with load at the joint. This allows using a smaller motor, when compared to other regulation techniques acting directly on the elastic elements. Another characteristic of ARES mechanism, is that the rolling point, in contact with the inner face of the slotted bar, can spin and roll a small distance when a deflection occurs thus not bending forces are transmitted to the spindle of motor M2. No oversize in the transmission element of M2 is required because no force other than the axial for regulating the slide position is expected.

\subsubsection{Force Sensor}

Robots and actuated systems that interact with human, must have the capability of detecting the forces and control them, along with controlling their position in the work space. By forcecontrolling the joint, a dynamic control of the joint in order to adapt to changes in environmental conditions is possible. To maintain a reduced size and dimension, the force sensor in ARES is embedded in the mechanism. Utilizing the same elements that give the compliance to the joint, torque measurements that may vary from approximately $-17 \mathrm{Nm}$ to $40 \mathrm{Nm}$ for a child of around

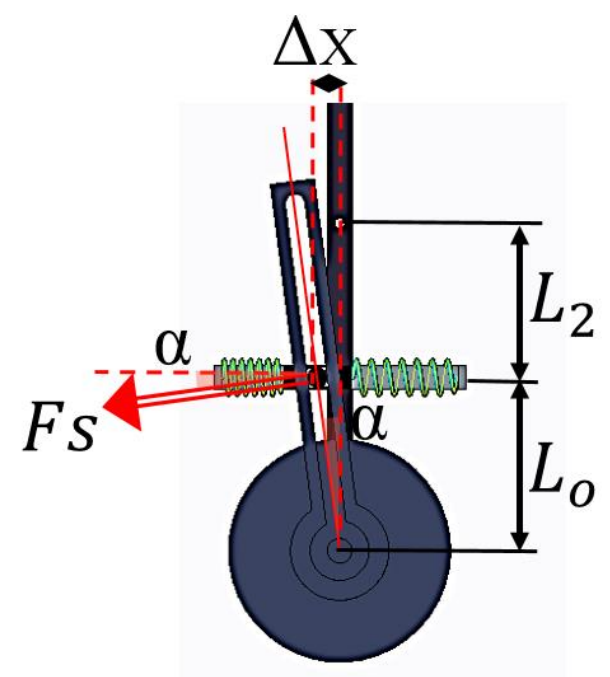

Figure 5-4. ARES Force Diagram. 
$35 \mathrm{~kg}$ at a speed of $6 \mathrm{~km} / \mathrm{h}$ are performed without adding bulky extra elements such as torque sensor. Moreover during swing phase to take advantage of the potential and kinetic energy by incorporating a zero-force control, the sensor has to be able of detecting small torques to avoid extra energy drain in the motors. The proposed design incorporates a rotary encoder capable of translating the linear displacement to measure the elastic element's compression $\Delta \boldsymbol{X}$, when a torque is applied at the joint, and using the actuators characteristics and the relationships below, a torque measured can be computed.

In Eq. 5-1, the basic relationship that allows us to calculate the torque exerted at a joint is shown.

$$
\tau=\bar{b} \times \bar{F} S
$$

Analyzing with the help of Figure 5-4, some aspects are exalted:

- The effective arm length $\overline{\boldsymbol{b}}$, when no deflection is present will match the distance $\boldsymbol{L}_{\mathbf{0}}$. The motor M2 controls the distance between the joint axis and the slider element that contains the elastic elements, thus $\boldsymbol{L}_{\mathbf{0}}$ can be modified by tuning its position.

- When the springs compress a distance $\Delta \boldsymbol{X}$, a deflection $\boldsymbol{\alpha}$ occurs. The position of the slider element remains still, but the arm length $\bar{b}$, will slightly increase. The rolling pivot $-\mathrm{S} 1 \mathrm{P} 1$ in the complaint mechanism will have rolled along the slotted bar-S1B1 because the elastic elements cannot bend in presence of the springs linear guides thus,

$$
\bar{b}=\frac{L_{0}}{\cos (\alpha)}
$$

Eq. 5-2

- The contact of the inner face of the slotted bar-S1B1with the rolling pivot -S1P1 is always tangent. The force $\overline{\boldsymbol{F s}}$, transmitted through the slotted bar $-\mathrm{S} 1 \mathrm{~B} 1$ will be orthogonal to the arm $\overline{\boldsymbol{b}}$ and the angle between the elastic elements' axis and the force equal to the deflection $\boldsymbol{\alpha}$. Hence, knowing the springs properties and the compression $\Delta \boldsymbol{X}$,

$$
\overline{F S}=\frac{2 . \Delta X . K}{\cos (\alpha)}
$$

Finally, replacing Eq. 5-2, and Eq. 5-3 in Eq. 5-1, we obtain a relationship for computing the torque at the joint taking advantage of the compliance of the actuator, 


$$
\tau_{\text {joint }}=\frac{2 \cdot L_{0} \cdot \Delta X \cdot K}{(\cos (\alpha))^{2}}
$$

Where,

$\Delta \boldsymbol{X}$, corresponds to the elastic element's compression, measured by the linear encoder.

$\boldsymbol{\alpha}$, the joint deflection due to the elastic elements compression.

$\boldsymbol{K}$, is the equivalent rigidity of the elastic elements in the slider element.

$\boldsymbol{L}_{\mathbf{0}}$, is the distance that can be adjusted by M2, between the joint axis and the slider device.

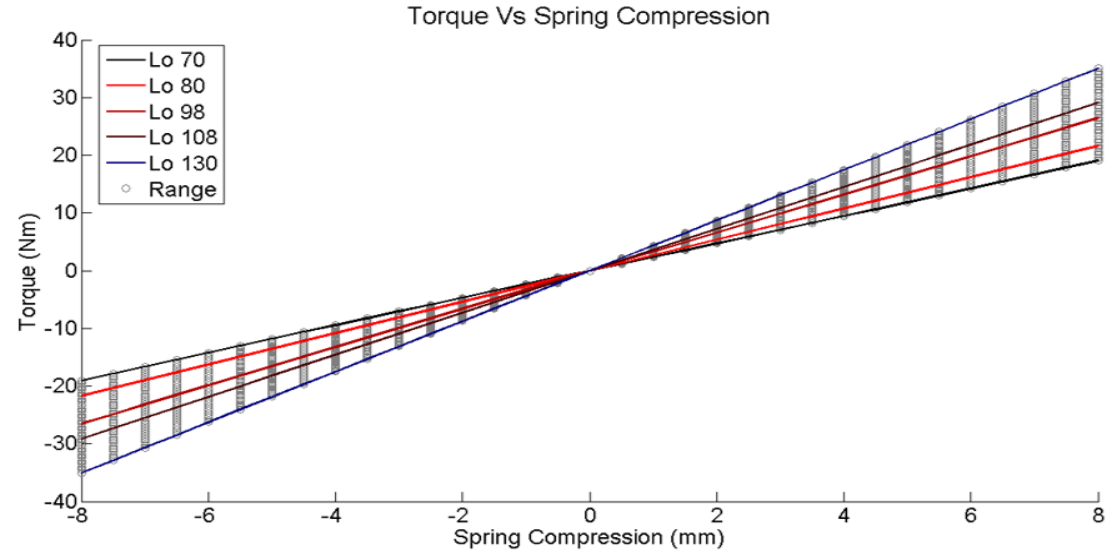

(a)

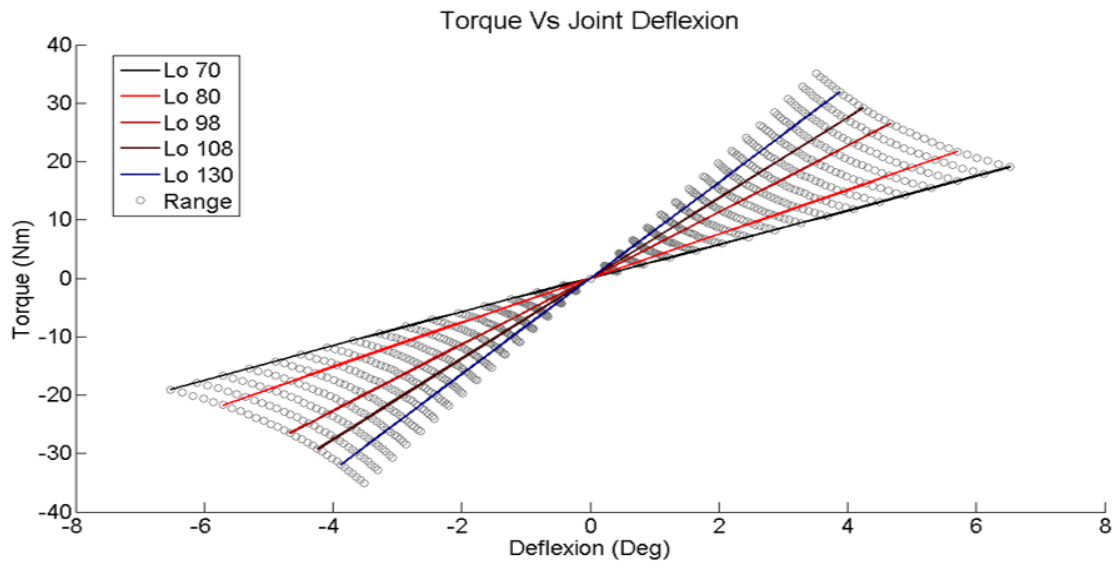

(b)

Figure 5-5. ARES Range of Stiffness. a) Torque Range for Different Positions of Lo b) Stiffness Range for Different Positions of Lo. 
The torque/force measurement is tight to the compliance of the system, by lowering the stiffness of the joint small torques can be detected and if higher torque values needs to be commanded, the system can be stiffened and the range of measured torques increased. In Figure 5-5, the range of torques and stiffness for the design parameters is shown. The maximum deflection achievable by this system is about $8 \mathrm{deg}$, at the minimum Lo position.

\subsubsection{Simulation-Based Force Sensor Evaluation}

The actuator prototype was designed with a set of constrains that intended to achieve a good force measurement by taking advantage of the elastic elements needed to introduce compliance at the joint. To evaluate the proposed working principle a series of simulations were performed with the help of Mechanism-CAD Software. A simplified model, containing the main elements of the actuator was introduced into the software. Some parameters considered in the simulations are listed below,

- Mass properties and values were kept close to those in the real prototype.

- The coupling between the stiff set and the compliant mechanism, was modeled as a contact point between the face of the rolling point, and the inner face of the slotted bar.

- In order to measure torques up to $40 \mathrm{Nm}$, springs pre-compressed $10 \mathrm{~mm}$ with a $\mathrm{K}=16.8 \frac{\mathrm{N}}{\mathrm{mm}}$ were placed into the simulation.

The dynamic behavior of the actuator was simulated when a commanded torque was applied at the joint and the compression of the springs were measured and logged as the data

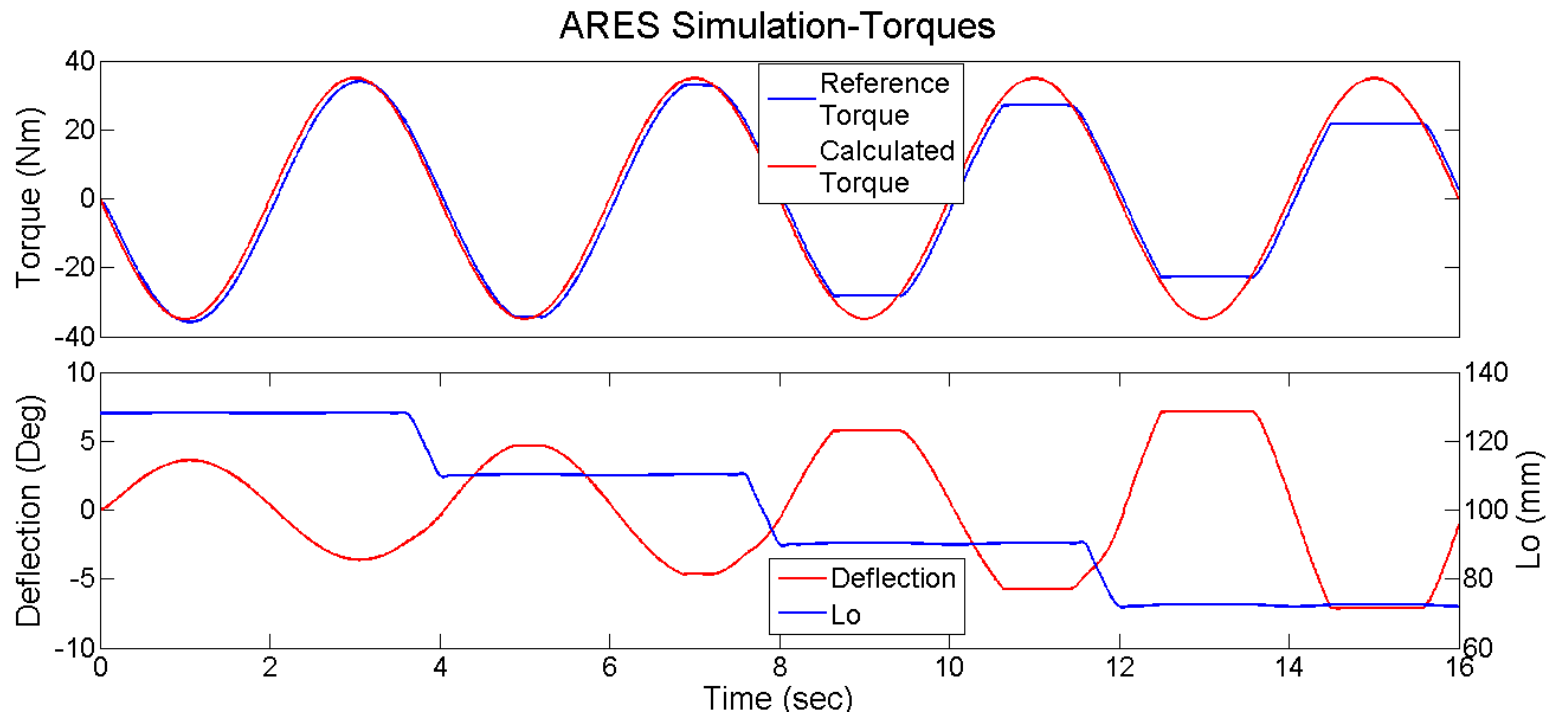

Figure 5-6. Open-Loop Tracking Simulation with Saturated Measurement 
expected to be collected with the encoder in the pivot -S1P1. In Figure 5-6, a sinusoidal torque of $\pm 36 \mathrm{Nm}$ was exerted to the joint, and the distance $\boldsymbol{L}_{\mathbf{0}}$, consecutively adjusted every 4 sec intervals. In top figure, the calculated torque using the relationship given in Eq. 5-4, and the logged data (springs compression and $\boldsymbol{L}_{\mathbf{0}}$ ) is contrasted with the commanded torque. The torque tracking in open loop fits to the commanded, but saturation can be observed for the lower values of $\boldsymbol{L}_{\mathbf{0}}$.

The compliance of the device is tight to the embedded force sensor range, this saturation is expected as the springs present a maximum deflection. In bottom figure, the deflection reaches its maximum for the last 2 positions of the sliding element at certain torque, lower than the magnitude of $36 \mathrm{Nm}$, here the springs are compressed at its maximum thus the torque measurement gets truncated. However, when high torques are desired, by setting a proper distance $\boldsymbol{L}_{\mathbf{0}}$, smaller deflections will occur, than those experienced at low values of $\boldsymbol{L}_{\mathbf{0}}$ (Low Stiffness) for the same torque. This arm distance adjustment, allow the range of torque that can be measured to increase.

A different simulated-evaluation is shown in Figure 5-7, compressions up to $0.2 \mathrm{~mm}$ are expected to be registered by using the linear encoder. Very low torque of $\pm 6 \mathrm{Nm}$ was commanded to the joint, adjusting the distance $\boldsymbol{L}_{\mathbf{0}}$ every 2 seconds. In this test, small compressions were registered and measured, as can be seen in the bottom figure. The open loop torque tracking shown in the top figure, presents better fit to the reference when the distance $\boldsymbol{L}_{\mathbf{0}}$ is low, thus the

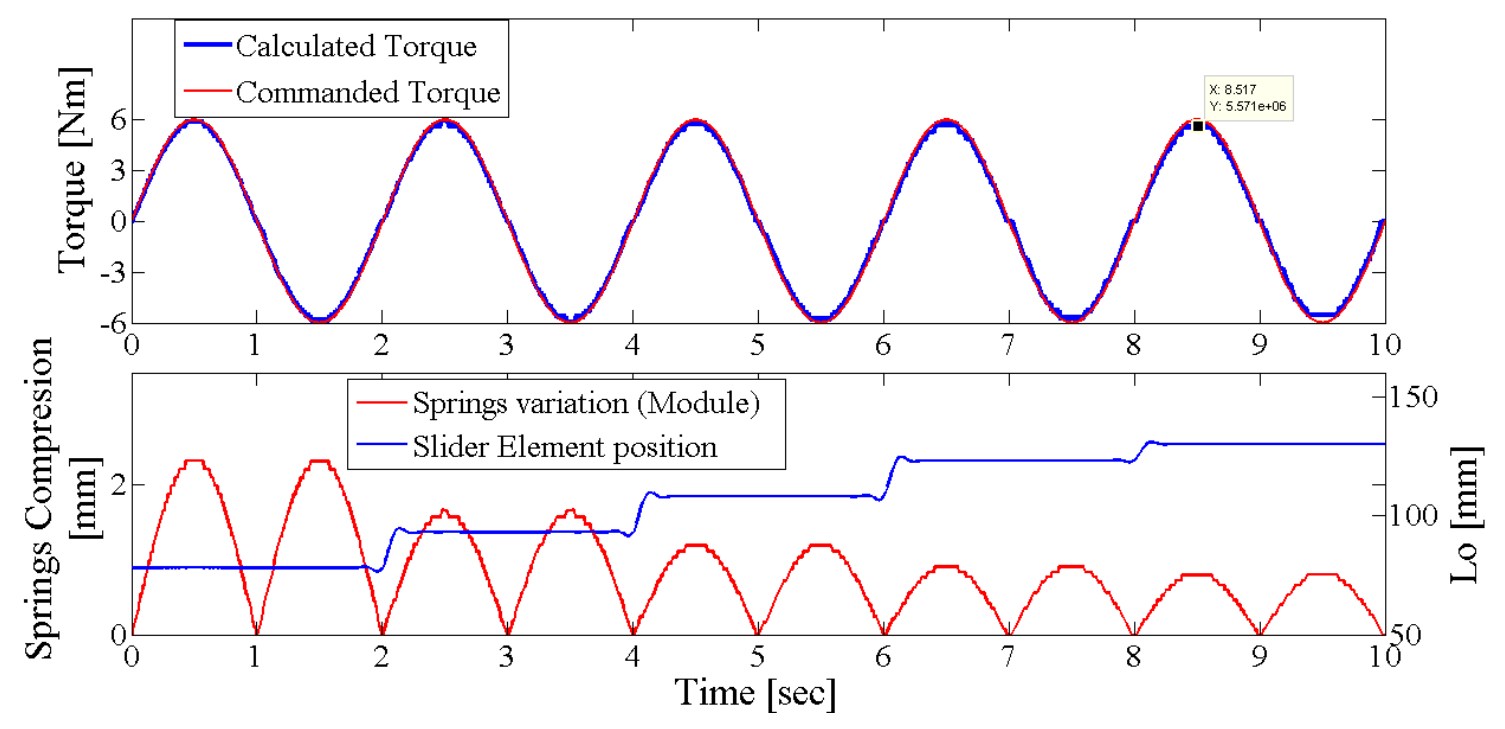

Figure 5-7. Low Torques Tracking Simulation in Open-Loop 
compliance high. The proposed actuator is intended to be torque controlled, and will try to exploit the natural dynamics of the legs during walking. A proper adjustment of the compliance of the actuator will allow us to detect small torques, adjust the torque range or set the joint stiffness when required in the performed task. The relationship between the joint compliance and the force measured sensibility and range could result in a wider bandwidth when the close loop force-control gets implemented if an appropriate match of compliance and exerted torque is made.

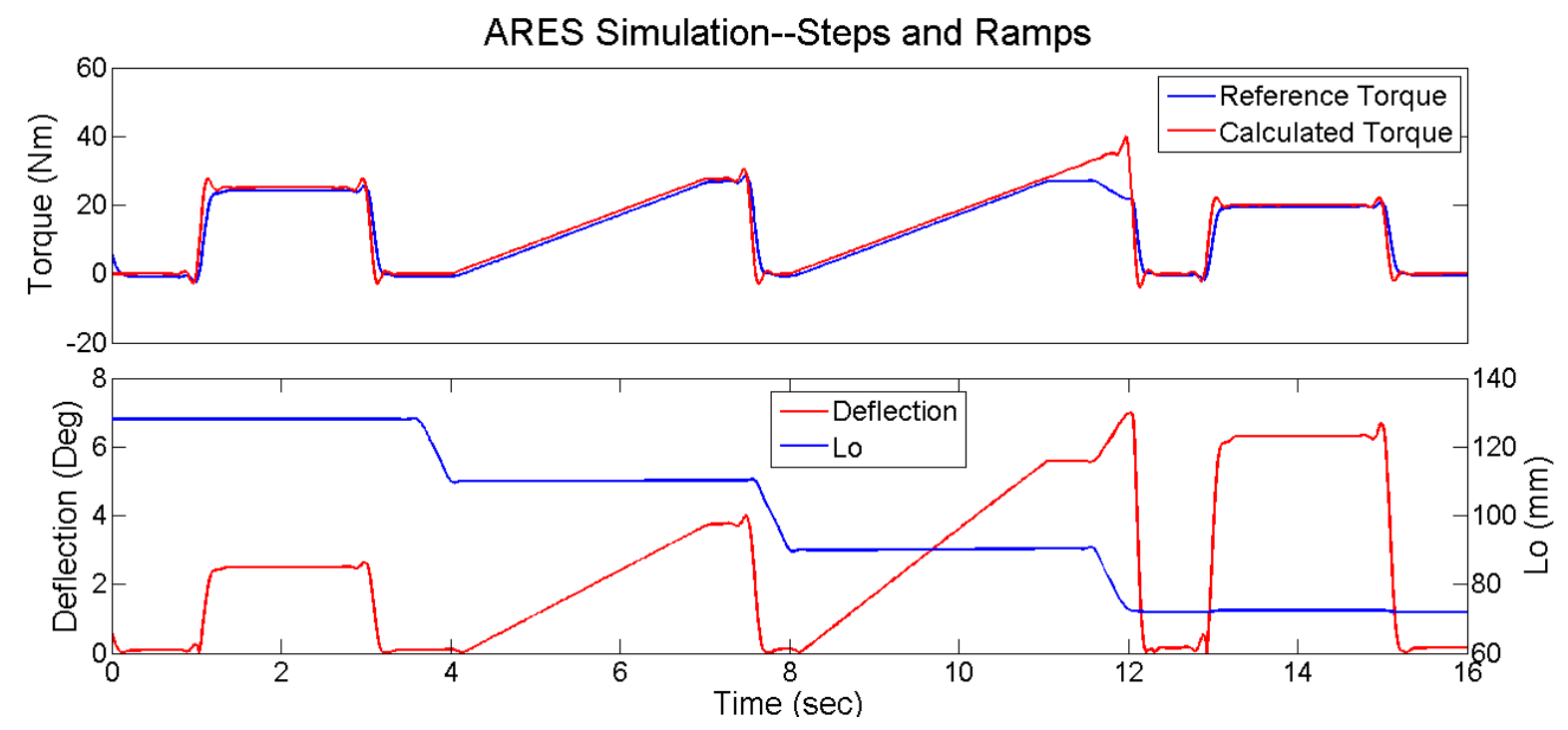

Figure 5-8. Open-Loop Tracking Simulation with Ramps and Steps Inputs.

A different simulation was tested and it is shown in Figure 5-8. Different stiffness levels are suitable to measure some torques, experienced larger or lower deflection. A trade-off between this characteristic can be useful during the locomotion cycle. High torques with large deflections can be implemented to achieve adaptation to the ground, like during loading response. Low deflections can translate in more energy directly transmitted to the joint, beneficial in the initial swing or even during toe-off at the ankle.

\subsection{ARES-Joint Prototype}

Following the mechanical design proposed in this chapter, a prototype of the compliant joint was built. This chapter addresses the details of the experimental setup to validate the embedded force sensor mechanism. Based on the results from the simulations presented in the previous Chapter, the actuator prototype was built trying to improve the contacts points, linear 
guide's displacements and exploit the maximum compression allowable by the elastic elements to have a wide range of force measurement.

The general specifications of the Adjustable Rigidity Actuator with Embedded Sensor built are presented in Table 5-2. In Figure 5-9, the main CAD-views of the Actuator are presented with some of its main dimensions. The ARES prototype, intended to provide the maximum deflection possible by the mechanism's geometry, combined with a wide range of stiffness that could be utilize in robotic applications intended for children.

Table 5-2. ARES General Specifications

\begin{tabular}{ll}
\hline \multicolumn{2}{c}{ Compliant Joint Properties } \\
\hline Peak Torque & Up to $76 \mathrm{Nm}$ \\
Max Deflection & $\pm 8^{\circ}$ \\
Range of Stiffness & $2.5-13 \mathrm{Nm} / \mathrm{deg}$ \\
Stiffness Adjusting & $0.25 \mathrm{sec}$ \\
time (Teo) & $1.2 \mathrm{Kg}$ \\
Weight & $260 \mathrm{~mm}$ \\
Length & $80 \mathrm{~mm}$ \\
Width & $90 \mathrm{~W}$ \\
Power
\end{tabular}

In Figure 5-10 the actuator prototype is presented and some of the main components are shown. To improve some of the qualities of the mechanism and to avoid significant friction losses or locking in the system, the following additions were incorporated.

- The Pivot -S1P1, coupled point between the main mechanisms, presents a cylindrical shape that tries to achieve a tangential contact between the slotted bar and the pivot, thus the transmitted force to the pivot is near to a radial direction 

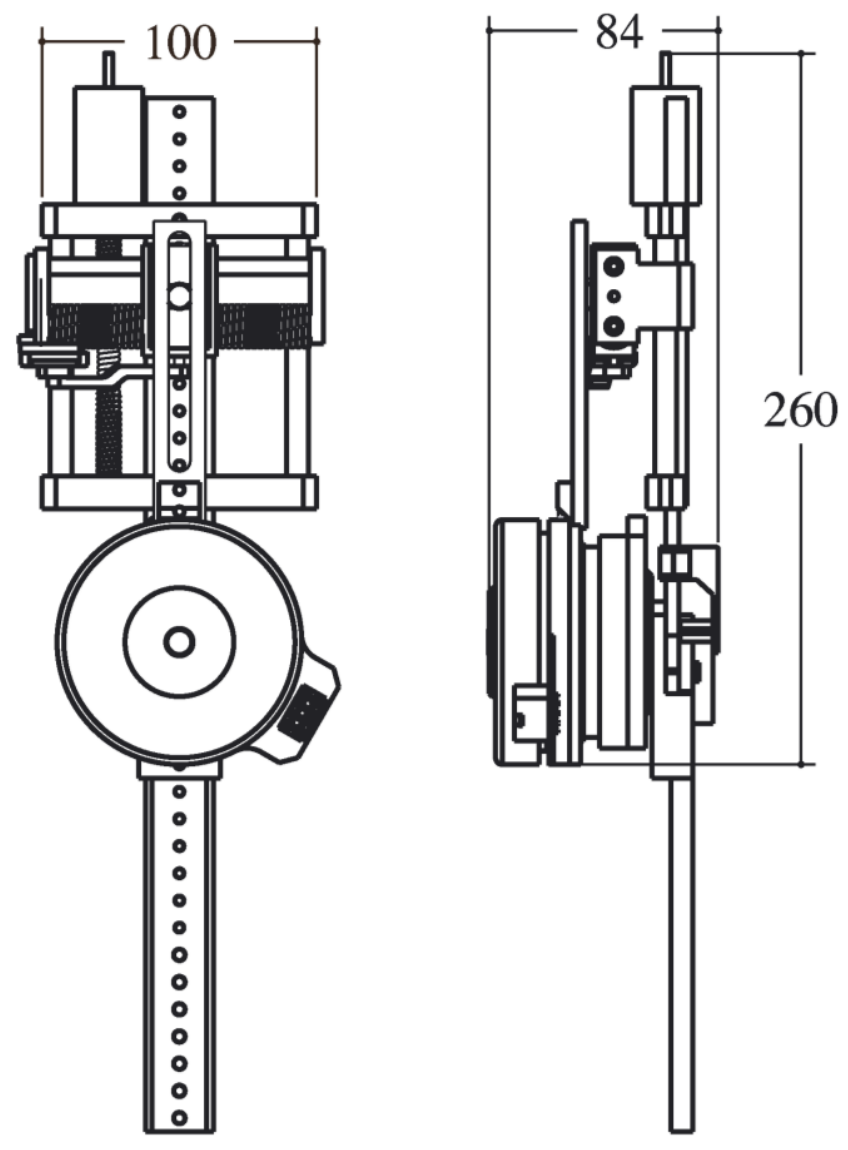

Figure 5-9. ARES Joint- CAD Model with Main Dimension.

force. The pivot Point is made of steel as all the forces are transmitted to it. S1P1 can spin to reduce friction while adjusting the elastic Element position.

- The Slotted bar-S1B1, is a slender bar made of steel with smooth and oiled inner surface.

- Linear guides are placed in the springs; the element where the sliding pivot is inserted has two linear bearings which constrain the spring compression, allowing just the displacement in one direction.

- Two steel bars in the Compliant Frame guide the elastic mechanism and support the reaction force due to torques exerted at the joint. There is no lateral stress expected 
at the Ball-Screw, as the linear guides y the Compliant Frame are the responsible for supporting the forces.

Most of the prototype is made of aluminum to reduce weight, the encoder and linear strip housings were made with a 3D printer as no resistance is need in those elements. Two bars were constructed to resemble the femur and the shank, and connected in a pivot point that resembles the knee joint, in the CAD-design in Figure 5-9, the stiff set is attached to the joint.

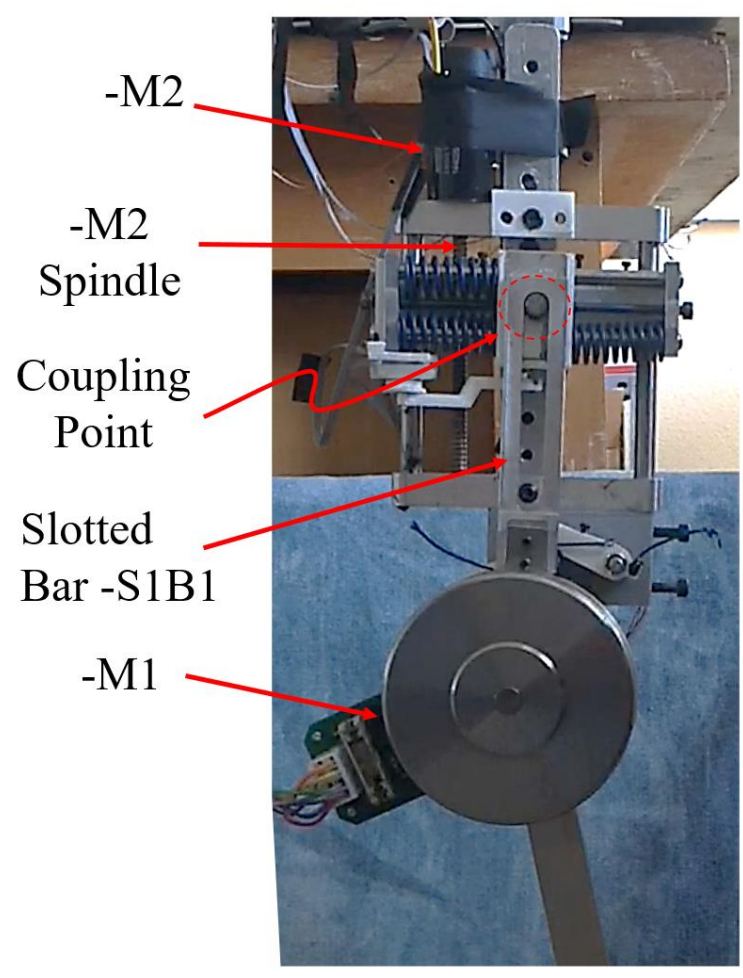

(a)

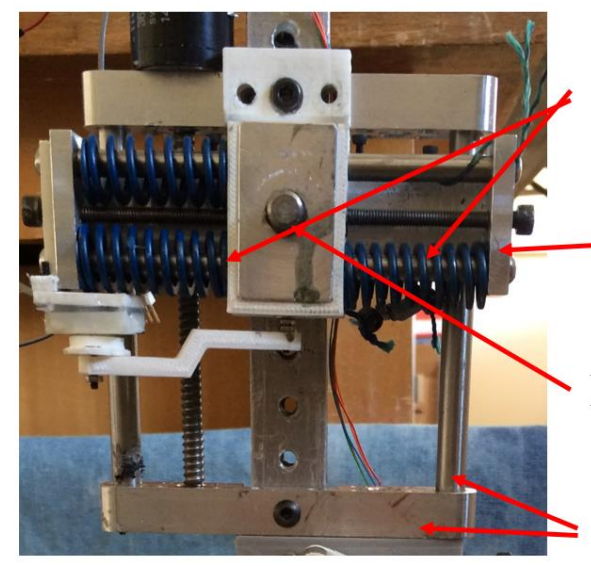

b)
Elastic Elements Sliding Element<smiles>[AlH2][AlH2][AlH2]</smiles>
Fixed frame and Linear guides

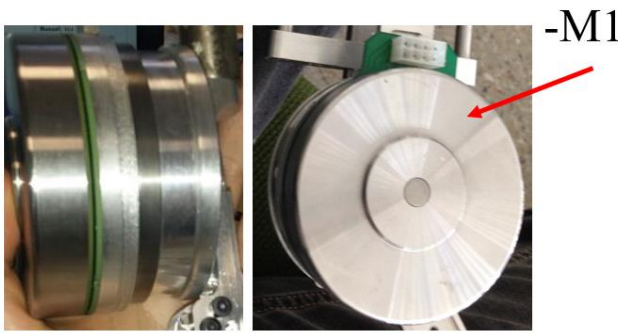

(c)

Figure 5-10. ARES Prototype.a) ARES Prototype-Front View. b) ARES Complian Mechanism' Prototype-Front View. c) Main Actuation -M1. 
The actuator prototype (In Figure 5-10) is intended to be incorporated into the ATLAS's knee for torque controlled motion and to increase energy efficiency by taking advantage of the adjustable joint compliance during the different phases of the locomotion cycle. To exploit the maximum compression allowable by the springs in the compliant mechanism, adjustments of the slider device' position has to be made dynamically during operation in order to adapt to the task requirement. In this section the evaluation of ARES and the behavior of the embedded force sensor are presented for different positions of the slider device and different loads at the joint.

\subsubsection{Experimental Setup}

The joint is instrumented with some components capable of logging a wide range of data during experimentation. A Test-Bench was built to put the joint through different conditions that allow the validation of the embedded sensor, and evaluation of the joint intrinsic characteristics.

\subsubsection{Test-Bench}

To evaluate the embedded force sensor in our actuator prototype, the system was incorporated at a realization of a knee joint conformed by two bodies pivoting at one end that resembles the knee joint, as shown in Figure 5-11. The upper link fixed to a grounded structure, maintains its vertical position during the experiments. A load at the joint is emulated by adding known weights at a given distance from the axis of rotation of the joint. This load is attached to the lower link of the structure, and by commanding different angular positions to the actuator, load variations can be exerted at the joint.
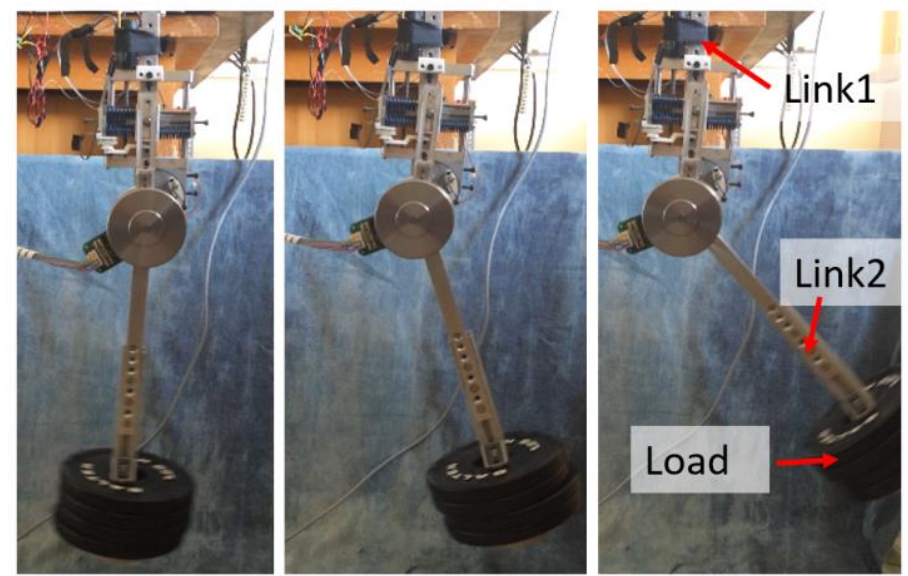

Figure 5-11. ARES Test-Bench Snaps. 


\subsubsection{Prototype components, electronics, and control.}

The system is formed by a set of mechanical and electronic components. The chosen elastic elements implemented to achieve the compliance are mid-load springs from Elemen Mat S.L. They are characterized by providing high load and an adequate compression to the application. To measure the compression of these springs, and the joint position, two absolute magnetic encoders (Austria Micro System AS5045) with custom boards are used. With joint position resolution of 0.09 degrees, and more than $0.2 \mathrm{~mm}$ of resolution for the linear encoder.

The data acquisition and control of these experiments were made with the help of Maxon motor controllers, Teensy 3.2 boards, and National Instrument Software. The main actuator of the prototype is a Maxon Motor of 24V, an ESCON 50/5 is used to control the main actuator -M1. This board was selected due to its nominal and maximum current outputs. To control the second motor-M2, controllers EPOS 24/5 were selected for its ability of positioning control.

A Master Slave configuration was implemented using 2 Teensy 3.2 boards, with processors of $72 \mathrm{MHz}$. The slave board is connected to the ESCON, and EPOS controllers. A PWM signal commands the joint position at the main actuator $-\mathrm{M} 1$, while an analog signal controls the position of the slider element by means of $-\mathrm{M} 2$. The data from the AS5045 encoders, along with current consumption, velocity, and position based on hall counts fed by the ESCON controller, are logged into the slave board. The master and slave are in continuous communication. Feedback data from the slave is sent via $\mathrm{i} 2 \mathrm{C}$ protocol to the Master, and transferred every millisecond to a main computer with National Instrument (NI) Software, The main characteristics of the system are shown in Table 5-3.

Table 5-3. Experimental Setup-Control

\begin{tabular}{lrr}
\hline Supply Voltage & $11-24$ VDC & -M1 Velocity \\
Nominal Current & $5 \mathrm{~A}$ & -M1 Current \\
Peak Current & $10 \mathrm{~A}$ & Outputs \\
Input -M1 & PWM Signal & -M1 Hall Signals \\
Input -M2 & Analog signal & Joint Position \\
& & Compression
\end{tabular}




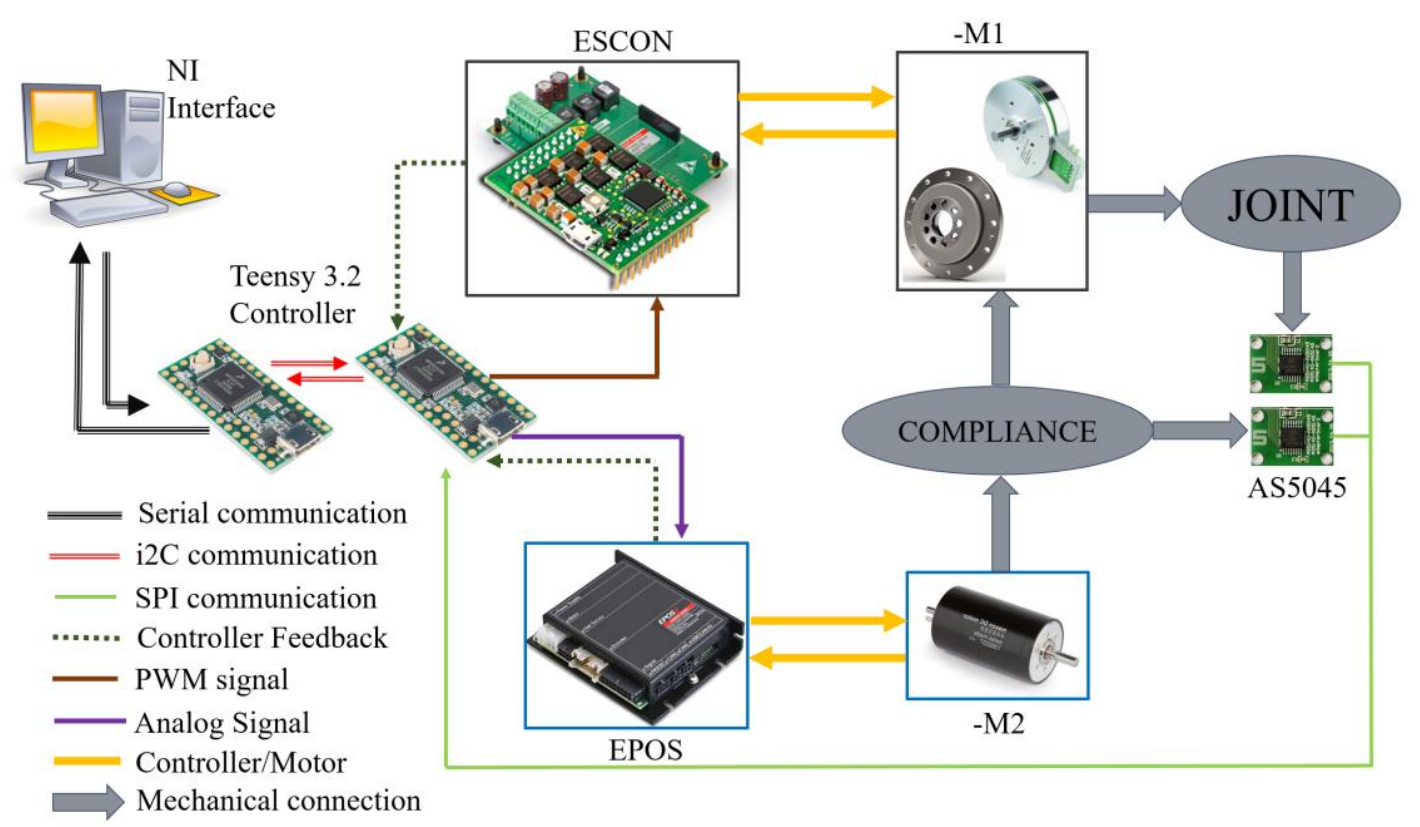

Figure 5-12. Simplified Schematic of the Experimental Control Setup.

\subsubsection{Performed tests}

In order to evaluate the main characteristics of the actuator several tests were performed. Being a force-controlled actuator, it is relevant to test the behavior of the embedded force sensor under different loads and stiffness levels as well. Also to exploit the actuators compliance, dynamic adjustments of the stiffness have to be made along its operation thus, evaluations of the stiffness adjustments were performed.

\subsubsection{Embedded force sensor-Torque measurement}

Different arm lengths were fixed during trials to achieve a variation in the joint equivalent stiffness by means of the compliant motor M2. The torque measured with the embedded sensor is a function of the elastic elements compression for a given arm length. The results obtained from different load weights are shown in this sub-section; first in Figure 5-13, with a load up to $9 \mathrm{~kg}$ attached to the shank of the Test-Bench, as shown in the experimental setup, a sinusoidal movement was commanded to the knee, through M1. The joint experienced a torque proportional to the load weight and the arm length projection. By measuring the spring's compression in the 
slider device and its separation from the joint axis, a torque measurement is calculated and contrasted with the load reference at the joint, given by the attached weight and the joint angular position.

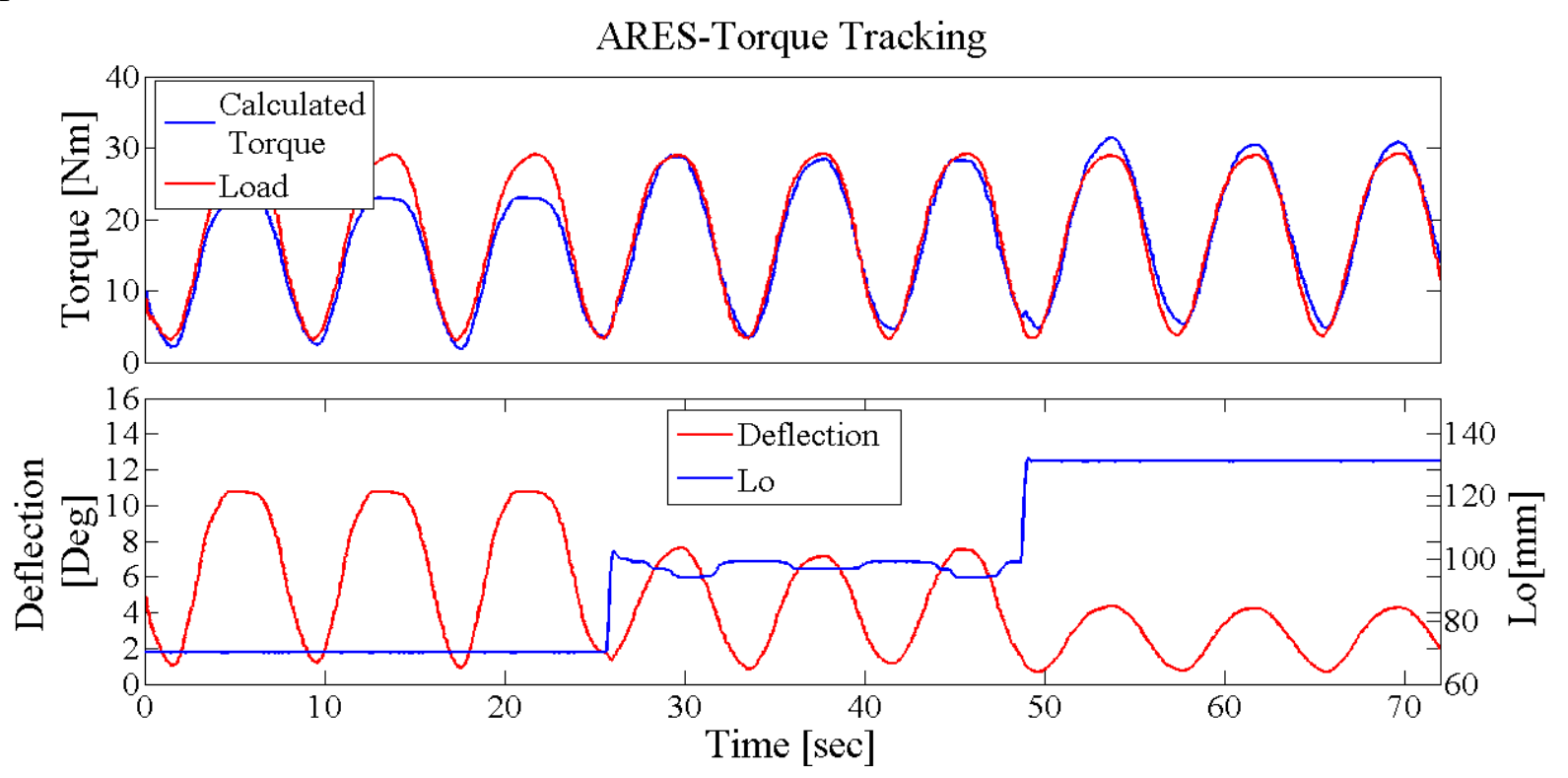

Figure 5-13. Open-Loop Torque Tracking. High Torque.

Three different levels of stiffness were commanded in this test. As can be seen in Figure $5-13$, it can be noticed that for the same torque, the deflection decreases as the slider device separates from the joint axis, thus the actuator stiffness increases, as well as the joint stiffness. In the minimum stiffness position, the deflections reach almost $10^{\circ}$. In the top figure, in the calculated torque, when the springs get fully compressed, as can be seen by the flattened peaks in the joint deflection, the measurement truncates. By adjusting the slider device position, the torque range that can be measured increases and the joint gets stiffer. A trade-off needs to be made between the desired compliance and the range of torque that needs to be measured.

Afterwards, the load at the lower link was replaced by a lower weight, around $2.5 \mathrm{~kg}$, and the experiment was repeated. In Figure 5-14, the results of this trial are presented; for low stiffness, the open-loop tracking fits closely to the reference. A peak for negative torques can be observed. This is due to the inertia of the load when going down and the joint changes the direction of the movement. However, for such low load, when the stiffness of the system increases, the compression in the springs decreases, having as a consequence that the torque tracking is no longer 
really precise. As more torque needs to be made to compress the springs, a delay in the calculated torque is observed for low torques at higher stiffness.

ARES- Low Torque Tracking
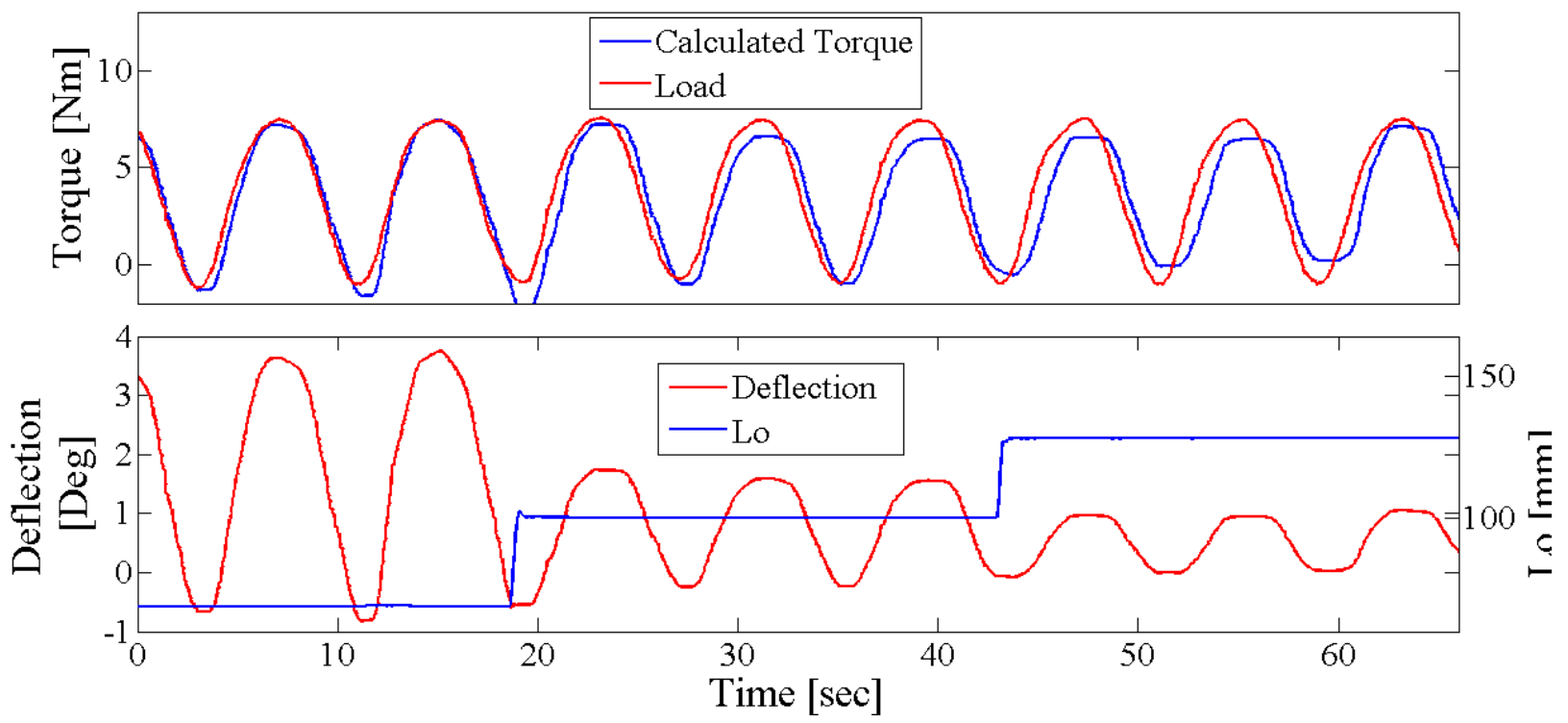

Figure 5-14. Open-Loop Torque Tracking- Low Torque.

The frequency of the sine reference was increased in another experiment, where with a load of about $2 \mathrm{Kg}$ at the lower link, the commanded motion will exert at the joint a dynamic load with significant inertial component. The results of this experiment are presented in Figure 5-15. Two different stiffness levels were evaluated, and it can be noticed that the tracking capabilities are adequate for the torque range applied at the joint. Figure 5-15 a) and b) show the results for High stiffness and Mid-Stiffness respectively. The torque tracking fits to the reference with the main difference of achieving this under different deflections. Figure 5-15 c) exhibits a comparison between the deflections experienced at a similar load with different levels of stiffness. This characteristic can be useful when different levels of adaptation are desired under the presence of similar loads.

In Figure 5-16, the calculated torque with the embedded sensor, as a function of the compression of the elastic elements when the compliant joint is deflecting is shown and contrasted with the torque reference. During the experiments for low torques, below $5 \mathrm{Nm}$; a fuzzy area is observed, where the reference data is affected by precision of the system used as reference. The striped data representing the torque reference gets more precise over $10 \mathrm{Nm}$. The torques measured with the actuator present a better precision when the arm length and the joint torque are low as the 


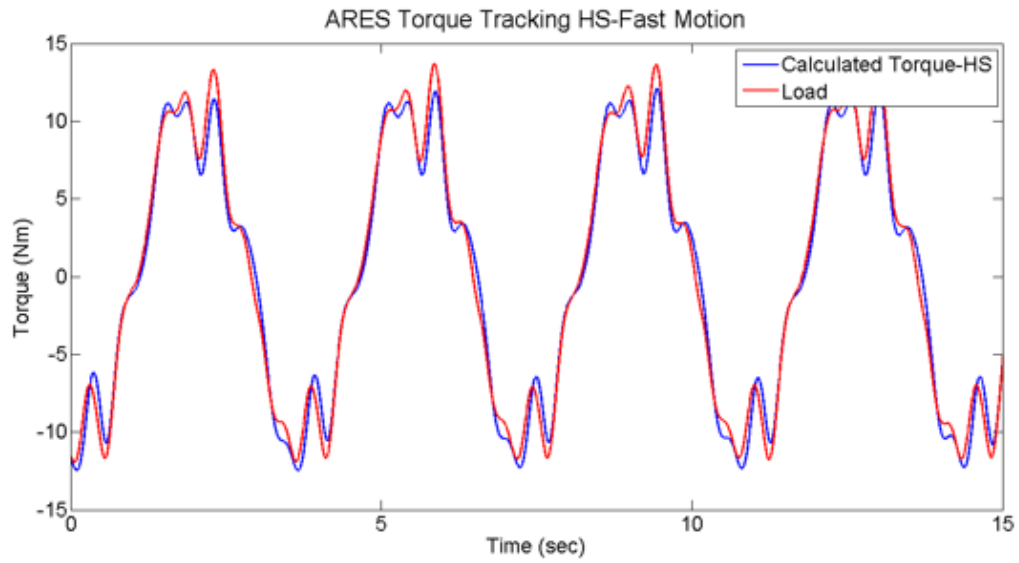

(a)

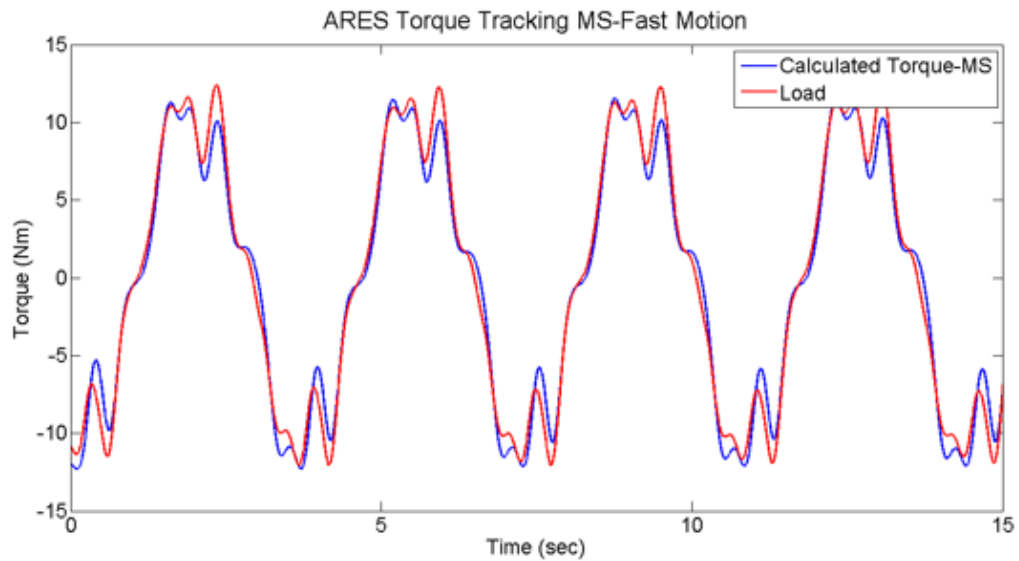

(b)

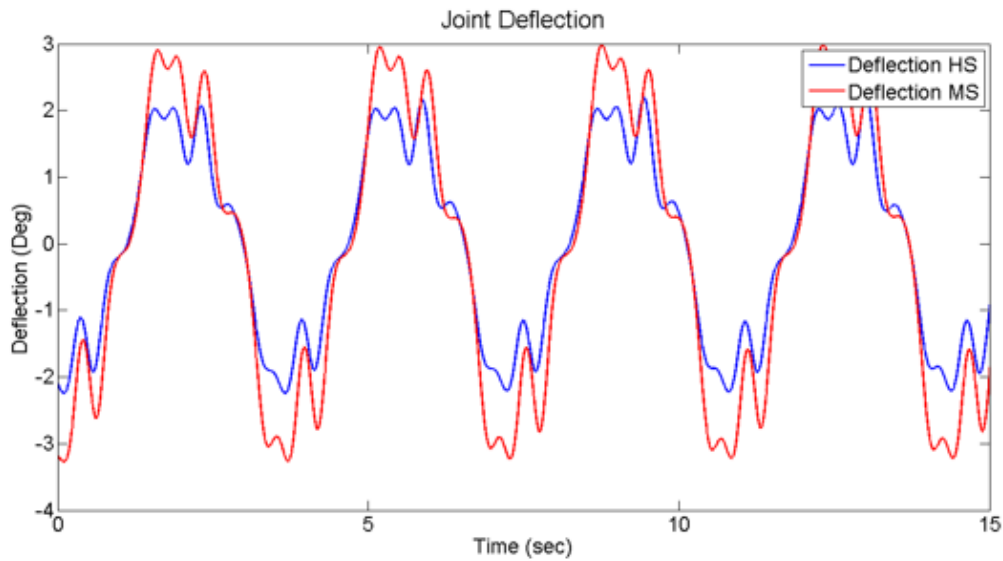

(c)

Figure 5-15. ARES Torque Tracking at Higher Frequency. 
linearity of the data for Stiffness $\mathrm{S} 2$ shows, and over the $5 \mathrm{Nm}$ fits to the reference different arm length and by adjusting the distance from S2 to S1, where Stiffness $\mathrm{S} 2<\mathrm{S} 1$. For lower stiffness, the slope of the system presents less dispersion on the data when compared to the higher slope associated with higher stiffness. These phenomena have been discussed all over the section, where lower stiffness presents better resolution to low torques, while larger stiffness should be used for measuring larger torques or allowing smaller deflections.

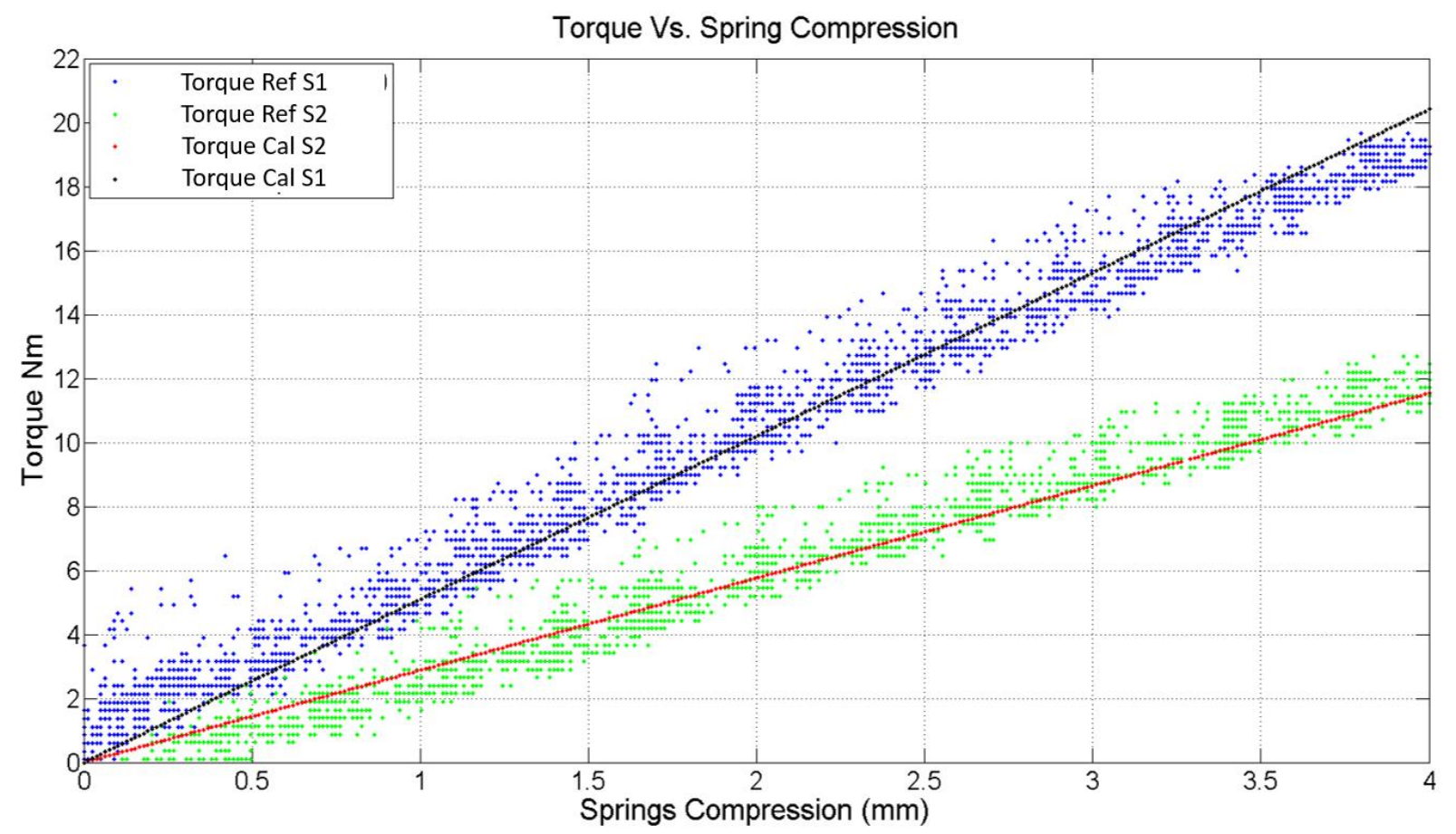

Figure 5-16. Torque Tracking Vs Spring Compression.

\subsubsection{Stiffness adjustment}

In order to fully exploit the compliance of the joint, and to adjust the measurement range to the adequate by the task been performed, the stiffness needs to be adjusted, not just dynamically but in very short time. ARES working principle, avoids that the forces transmitted to the springs act directly over the motor (-M2) responsible for adjusting the slider element position. This allows using a smaller motor to tune the stiffness of the system. In Figure 5-17 the time consumed by M2 to change the position of the slider device can be seen. In this test, a critical condition was evaluated, the stiffness regulation was commanded without significant external load at the joint. 
When a torque is applied at the joint, the deflection experienced by the system produce that a component of the force compressing the springs, acts in the motor M2 direction.

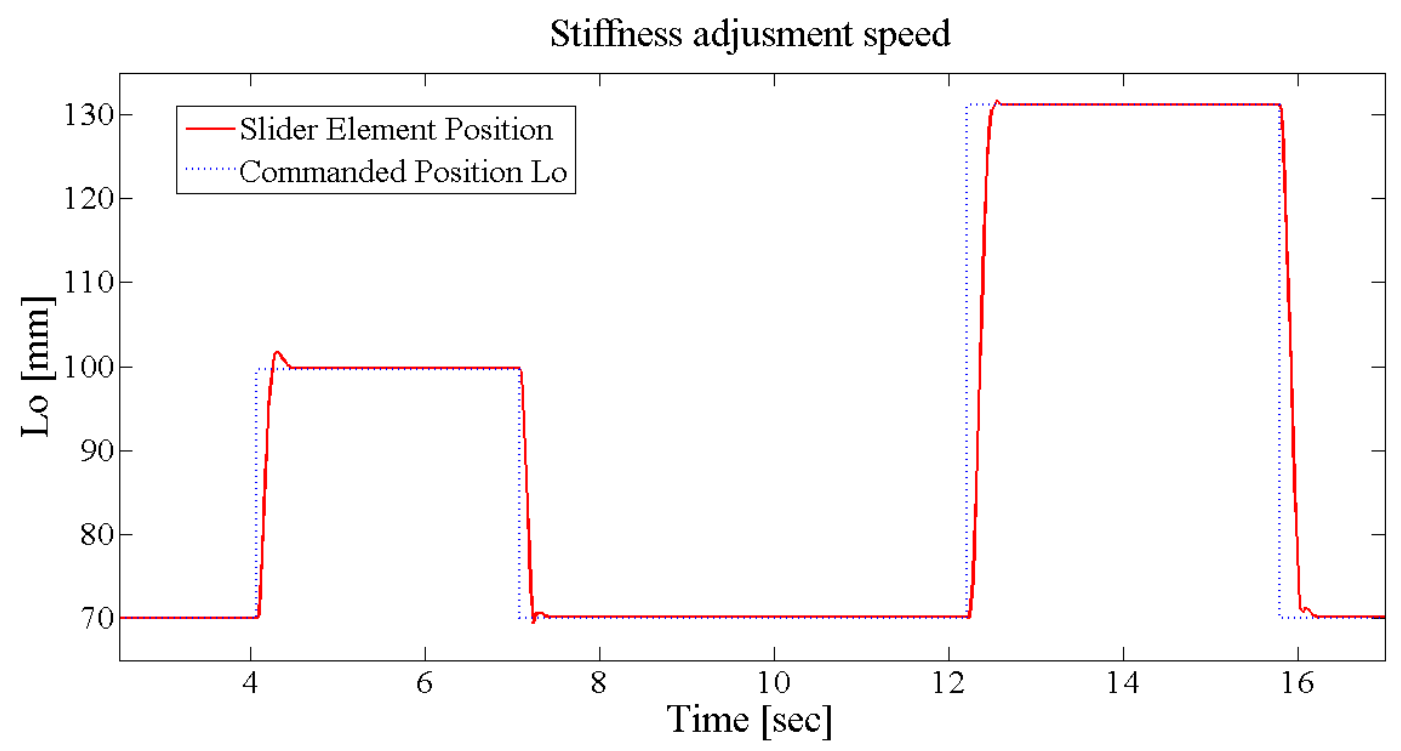

Figure 5-17. Stiffness Adjustment Speed.

The slider device position was commanded from its minimum to medium and then from minimum to maximum position allowed by our system. As a result, by having the combination of motor that can spin at more than 9,500 RPM and a ball screw with 2-mm lead, the rigidity adjustment occurs in a very short time. Many trials were made to have an average value of the adjustment time. The results indicate that from minimum stiffness to maximum stiffness our systems take a time of approximately 0.25 seconds, with linear-like behavior for intermediate stiffness values.

\subsubsection{Angle relationship - DOF in the system}

The compliant joint is a mechanism integrated by the stiff complex and compliant mechanism. The stiff motor in -M1, in combination with the deflection $\alpha$ due to spring compression in the presence of an external force or exerted torque determines the real joint position. Figure 5-18 shows the joint angles when a set of inputs, ramps, and consecutive steps were commanded to the M1 motor. The Hall sensor of the M1 motor gives the commanded angle 
(q); the rotary magnetic sensor at the knee allows us to know the joint angular variation $(\beta)$, and the spring's compression at the elastic mechanism for a known arm length provides us with the angular deflection $(\alpha)$ due to the exerted torques.

Angles variations

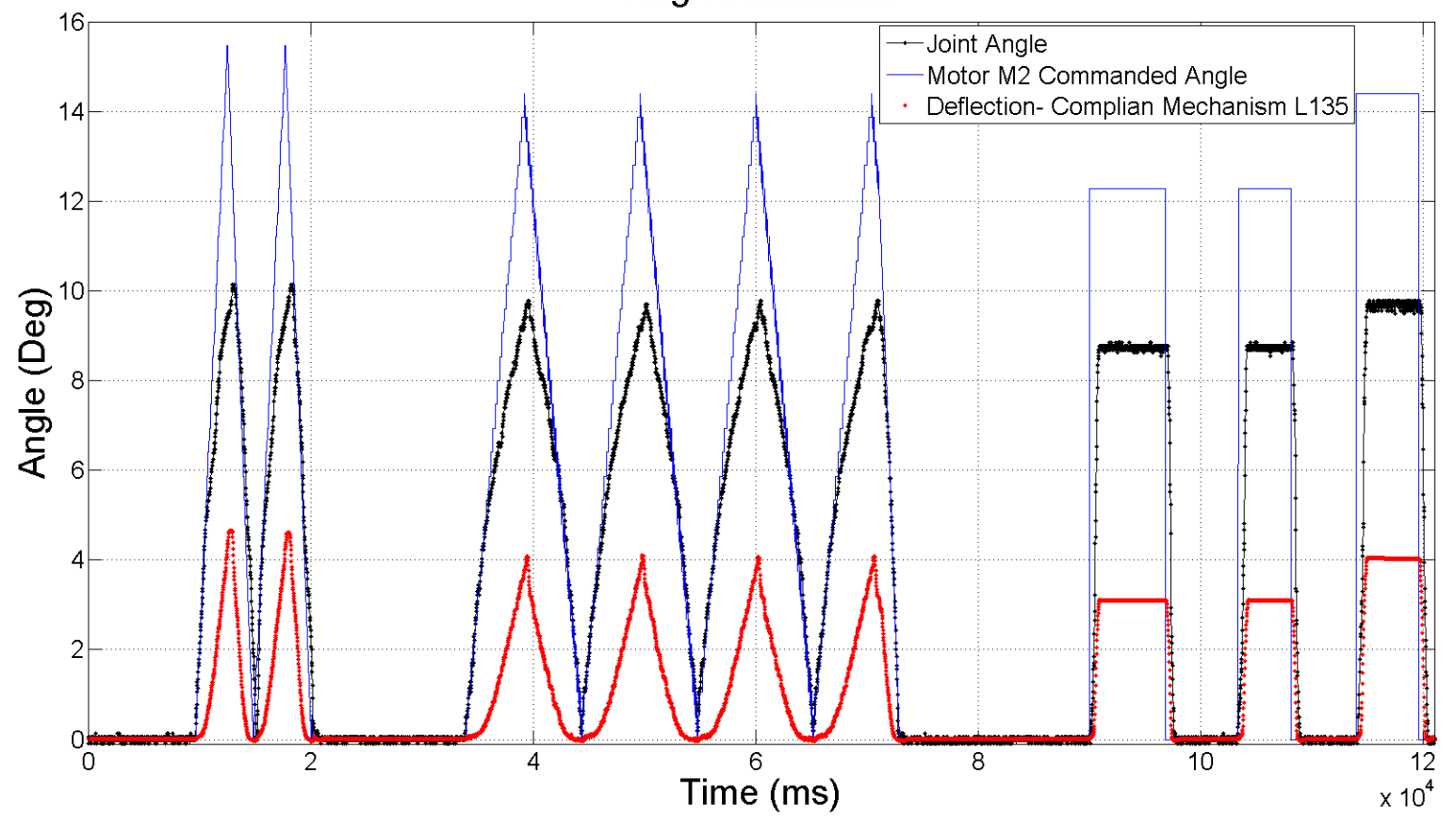

Figure 5-18. ARES Joint Angle Relationship.

As the system is coupled through the sliding pivot and the slotted bar, it is evident that the angle variations at the joint, motor, and deflection $\alpha$ are strictly related:

$$
\beta=q-\alpha
$$

Where:

$\alpha$, correspond to joint angle between upper link and lower link.

$q$ is the motor M1 angle at the HD output.

$\varphi$ is the deflection angle between the actuator and the joint, and it is a function of the displacement of the sliding pivot.

When no torque is applied $\varphi$ and $q$ are equal as can be seen at the beginning of the ramps inputs, likewise when step inputs were commanded, the deflection $\alpha$ at the joint can be appreciated quickly and no appreciable region with no torque acting can be seen. 
Table 5-4. Actuators Comparison including ARES

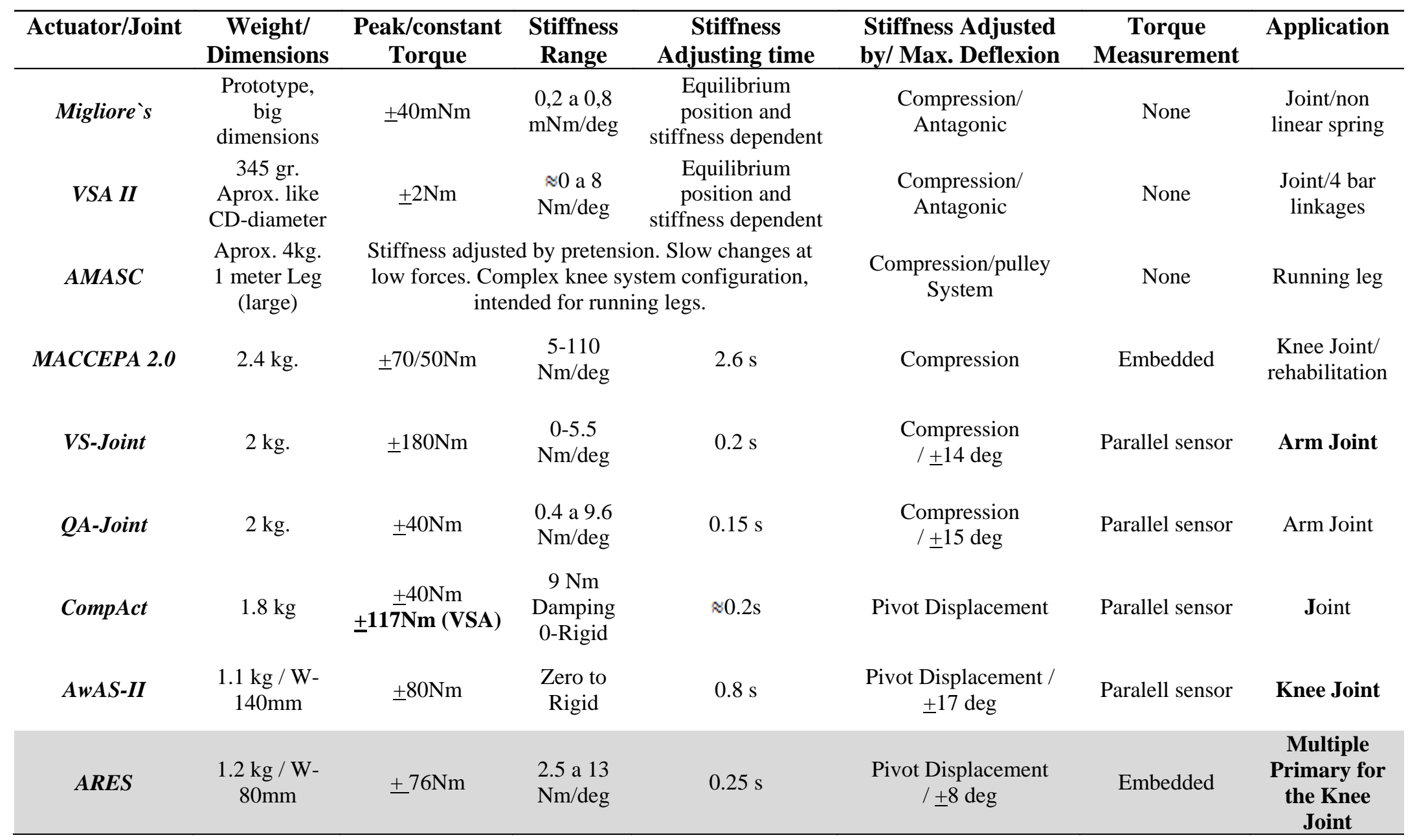




\section{Chapter 6}

\section{Actuator of Adjustable Rigidity with Embedded Sensor and Locking mechanism (ARES-XL)}

In order to maximize the ability to exploit the kinetic, and dynamic properties of the human biomechanics during the locomotion cycle, novel actuator system seek to be as versatile as the human muscle by providing some properties such as: (1) Adaptability and shock absorption. (2) Spring-like behavior. (3) Controlled-energy storage capabilities.(4) Fast torque generation (Impulse). An actuator with Adjustable Rigidity, Embedded torque Sensor, and locking mechanism (ARES-XL) is presented in this chapter, this actuator is an improved version of the original realization. ARES is capable of controlling stiffness and joint position independently by controlling the location of the springs along the slotted bar in the compliant mechanism. That system only allowed a limited amount of compliance, and the stored energy at the joint had to be used instantaneously after any change in the power direction occurred, sometimes even opposing the commanded motion. The novelty of ARES-XL resides on the implementation of a double bar linkage transferring the joint moment to a set of springs, in order to achieve the compliant behavior of the joint. As well as an add-on locking mechanism for storing energy in a particular configuration. This improved actuator version preserves several properties from the original ARES realization, and notoriously increases the joint compliance, and energy storing capabilities.

\subsection{ARES-XL Design.}

Focusing on the actuation of the ATLAS lower-limb active Orthosis, ARES-XL is intended to be implemented in several joints of the robotic device. Similar to ARES, the equilibrium position and its compliance are controlled independently. Torque-controlled motion together with maintaining reduced lateral size are main goals for this design, together with a wide range of capabilities that allow its implementation in different joints with different behaviors. The 
actuator's mechanics are presented below together with its working principles and torque sensing capabilities.

\subsubsection{Joint Mechanics}

A CAD representation of ARES-XL is shown in Figure 6-1, similar to ARES, this actuator is formed by 2 main components. A compliant mechanism, responsible for setting the joint stiffness and a stiff complex to generate the main power required at the joint. The stiff complex and the compliant mechanism are arranged along the structure in order to reduce the lateral size of the mechanism. An especial locking mechanism (add-on) can be implemented in ARES-XL in order to lock a certain joint deflection or store the elastic energy for later uses according to the joint requirements.

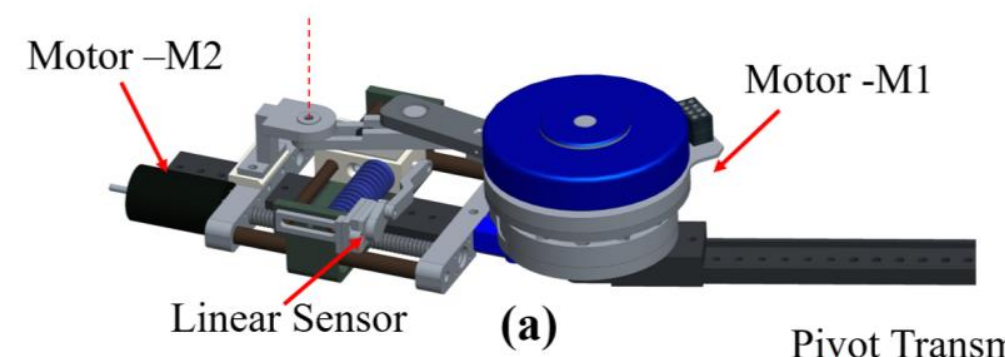

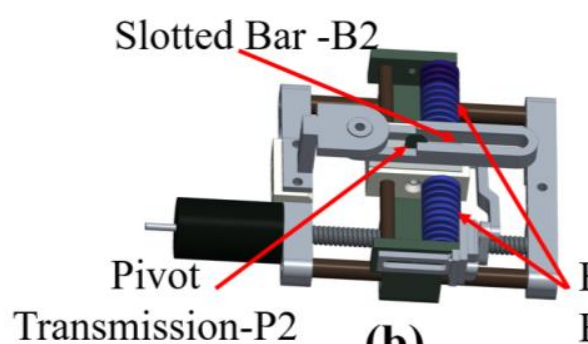

Transmission-P2 (b)
Elastic Elements (c)

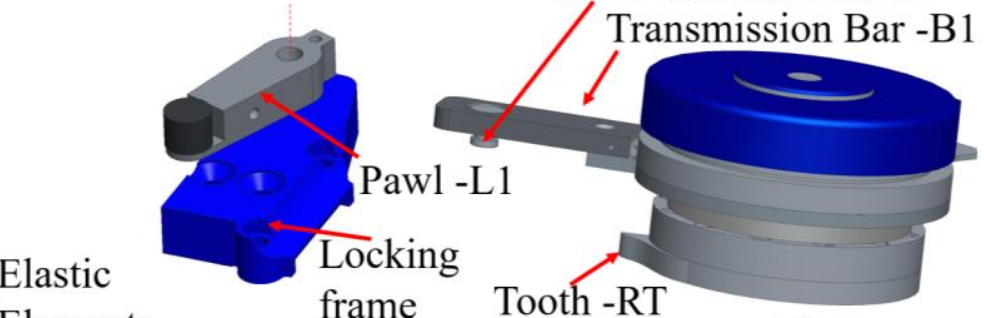

frame Tooth-RT

Pivot Transmission-P1

(d)

Figure 6-1. ARES-XL Joint Mechanics. a) CAD Drawing of ARES-XL, b) CAD of Compliant Mechanism, c) Locking Mechanism -Add on, and c) CAD of Stiff Complex.

\subsubsection{Stiff Complex}

The stiff complex presents a traditional combination of stiff actuator and a gearbox. The required high size/torque ratio during locomotion cycle and weight/torque ratios for this application, are achieved by the implementation of a flat Maxon 90W brushless DC motor in 
combination with Harmonic Drive unit with 100:1. The motor, (Figure 6-1 d)) and the Harmonic Drive combination, denoted as M1, control the equilibrium position of the joint, The combination integrated with the transmission bar-B1 and the pivot - P1, conform the stiff complex. The torque delivered or hold by the joint, is transmitted by the pivot -P1 to the compliant mechanism, through the connection with $-\mathrm{B} 1$, thus the length of this transmission bar $-\mathrm{B} 1$ is related to the force transmitted to the structure. Maxon Flat Motor was selected because of its reduced length and high torque, which makes it very suitable for exoskeletons and active orthosis applications. With a reduction of 100:1 ratio the nominal outputs of the stiff complex can go up to $2.5 \mathrm{rad} / \mathrm{sec}$ and $30 \mathrm{Nm}$, enough to achieve the requirements during the locomotion cycle in level ground at low speed, with certain limitations in sections of the gait such as swing and push-off, associated with velocity rates faster than those achievable by traditional electrical motors.

The mechanical structure of the stiff complex, incorporates a tooth $-\mathrm{RT}$ at a certain angle attached to the output of the complex, this element is intended for implementing the locking properties of the actuator.

\subsubsection{Compliant Mechanism}

This mechanism controls the amount of compliance of the joint. A set of elastic elements are placed in a slider system that is connected to a spindle drive for adjusting the slider position along the linear guides of a fixed frame, see Figure 6-1 (b). A Motor-M2, Maxon AC motor, RE-

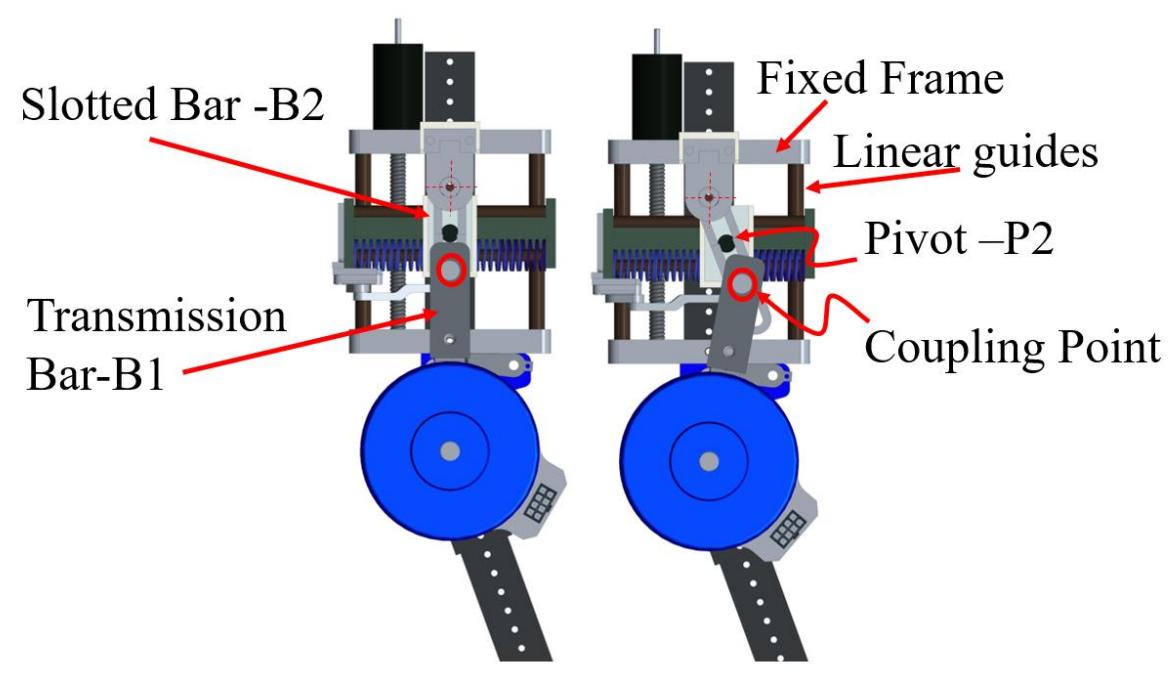

Figure 6-2. ARES-XL Components Coupling 
25 , of $20 \mathrm{~W}$ (considerably smaller that the main motor in the M1 complex) connected to the spindle drive exert the required force for varying the slider position when it is commanded. The slotted bar $-\mathrm{B} 2$ in one of its end can pivot over the fixed structure of the compliant mechanism, and the pivot $-\mathrm{P} 2$ transmits the force of the elastic elements to the inner face of $-\mathrm{P} 2$.

The pivot $-\mathrm{P} 1$ at the end of the transmission bar $-\mathrm{B} 1$, transfers the torque from the stiff complex to the inner face of the slotted bar $-\mathrm{B} 2$, this point of contact is defined as the coupling point between the compliant mechanism and the stiff complex, as shown in Figure 6-2.

\subsubsection{Locking Mechanism - Add-on}

In order to take advantage of the joint deflection in sections of the gait not directly connected, the ability to lock the position of the elastic elements in the compliant mechanism results useful. ARES-XL can incorporate an add-on device into the actuator structure that will allow locking of the deflection position, while still being able to modify the output joint position. Figure 6-1 (c) shows the device used for achieving a mechanical locking. The device can be categorized as a ratchet [43] that comprises a round structure with a tooth and a pawl that engages with the teeth and performs the locking.

\subsubsection{Working Principle}

This new actuator can be also classified based on the work of the VIACTORS consortium [62] and chapter 4, as an actuator with controllable transmission ratio. A double bar system is formed by the slotted bar-B2 and the transmission bar-B1 (Figure 6-2). Torques exerted at the joint are transmitted through the pivot transmission $-\mathrm{P} 1$ to the inner face of the slotted bar $-\mathrm{B} 2$. The reaction force is transmitted through the slotted bar to the pivot - $\mathrm{P} 2$ in contact with the elastic elements in the compliant mechanism. The force in the coupling point is related to the torque at the joint and the length of the bar-B1. Figure 6-3 shows that in the presence of torque at the joint, the force transmitted through - $\mathrm{P} 1$, is opposed by the elastic elements by the contact between the slotted bar $-\mathrm{B} 2$ and the pivot $-\mathrm{P} 2$. The distance $\mathrm{L}_{2}$ is directly related with the amount of compression in the elastic elements at a given torque to reach equilibrium.

The distance between the pivot axis of the slotted bar-B2, and the slider system containing the elastic elements is related to the amount of deflection $\alpha$ experimented at the actuator system at the same external or exerted torque. If the distance $\mathrm{L}_{2}$ is large, smaller deflection $\alpha$ for a given 

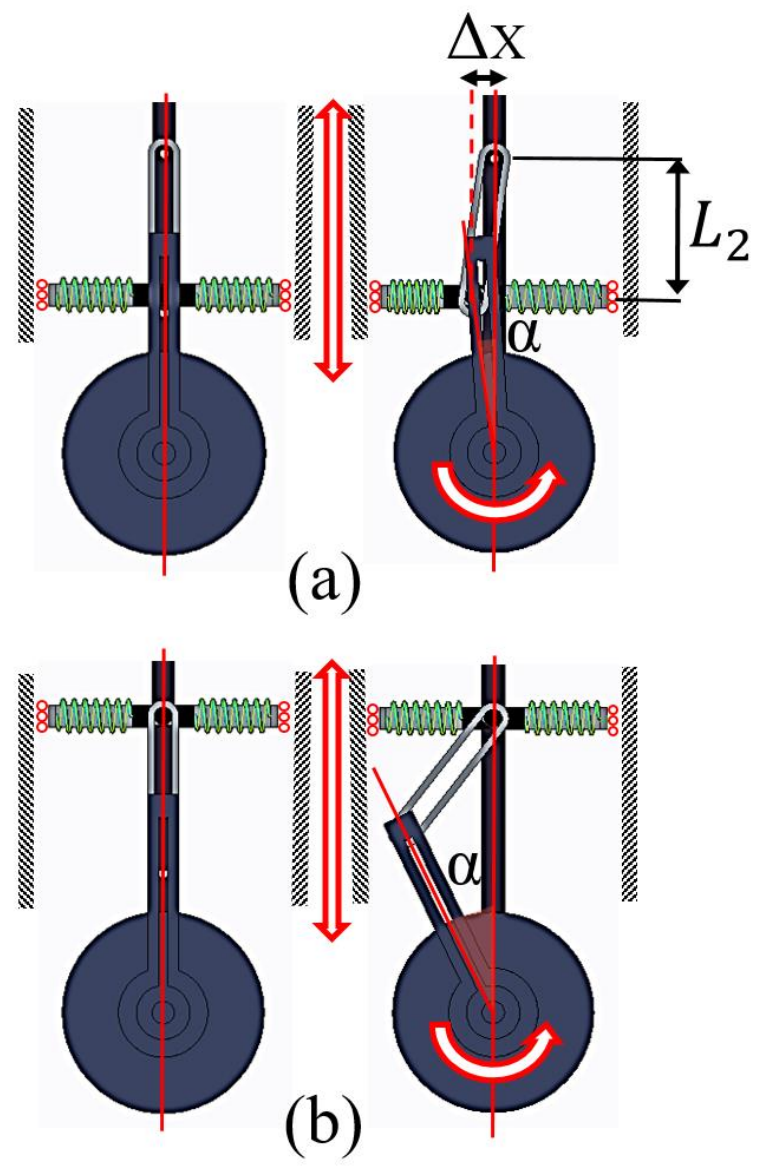

Figure 6-3. ARES-XL Working Principle. a) ARES-XL High Stiffness b) ARES-XL Minimum Stiffness.

torque at the joint can be seen. For $\mathrm{L}_{2}$ near 0, as can be noticed in the diagram in Figure 6-3 (b), the compliant mechanism does not oppose resistance to the transmitted force, thus the stiffness in that configuration is zero or close to zero. By controlling the position of the slider system with the use of the motor M2 in the compliant system, a variation of the output stiffness can be achieved, with the capability of achieving zero stiffness.

The controllable transmission ratio maintains the power necessary for the variation of the arm length low. The force produced by the motor M2 does not act directly on the springs, which allows using a smaller motor to set the position L2. The pivots -P1 and -P2 can slightly roll inside the slotted bar, the system tries to avoid bending of the elastic elements, and the force is directly transmitted to the fixed frame by the linear guides, this in combination with the transmission ratio 
principle, releases the motor -M2 and spindle from high loads, that can require oversizing of the mechanical components.

\subsubsection{Locking Principle}

The main purpose of this locking mechanism, is its implementation in a knee actuation. Based on chapter 3, the knee in the early phases can be seen as passive joint, with a rotational elastic element opposing to the body load. Compliant joints can mimic this behavior but at the end of the support phase, the joint torque changes direction maintaining the direction of rotation. Any energy stored at that point will be wasted and could even oppose the motion. ARES strategy was the increase of the stiffness in the system, thus the main motor will entirely supply the required power at that phase. By implementing the add-on locking mechanism in ARES-XL, at the end of the support phase the lock is passively engaged if the deflection is higher than a fixed value, around 10-15 degrees. The pawl's default position presses the round structure with the tooth attached to it. After the push off, the knee continuous to flex loaded in opposite direction to the locked position. The main motor will deliver the required power to move the joint that is not supporting the body weight during the swing phase. Figure 6-4 presents the sequence, where the lock is engaged (a)

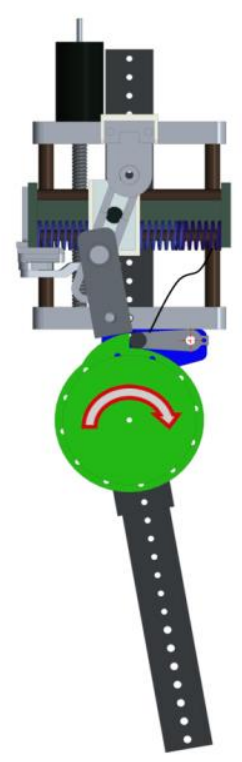

(a)

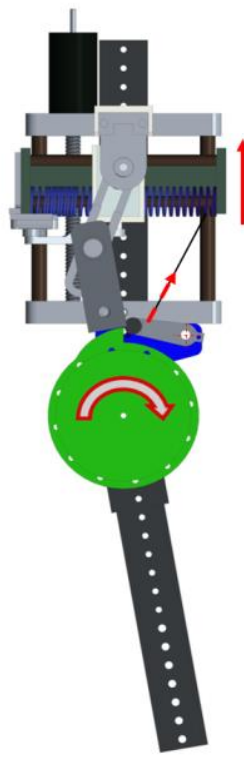

(b)

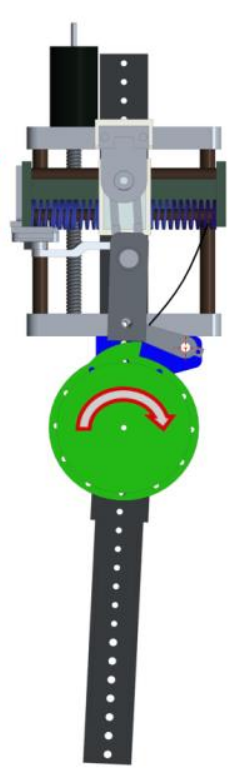

(c)

Figure 6-4. ARES-XL Locking Mechanism. a) Tooth and Pawl Engaged while Joint Loaded in Opposite Direction.

b) Slider Change Position, Pulling the Pawl to Disengage. c) Pawl Disengaged- the Joint Moved Freely Due to Load and Springs. 
the joint position can change keeping the deflection. At the end of the swing, (b) the slider from the compliant mechanism will unlock the joint by decreasing the joint stiffness and by pulling the pawl, moderately loaded. Once disengaged, and with low stiffness, (c) the weight at the link, the energy stored, and the deflection will cause the joint to swing freely without the main motor acting.

\subsubsection{Force Sensor}

The force sensor in ARES-XL is embedded in the mechanism. The elastic elements are used simultaneously to give compliance to the joint, as well as to achieve torque measurements without adding bulky extra elements such as a torque sensor. The system uses a magnetic encoder in order to measure the linear displacement of the elastic elements. The compression $\Delta \boldsymbol{X}$, in combination with the geometry, and mechanical characteristics of the system allow computing a torque measurement during operation.

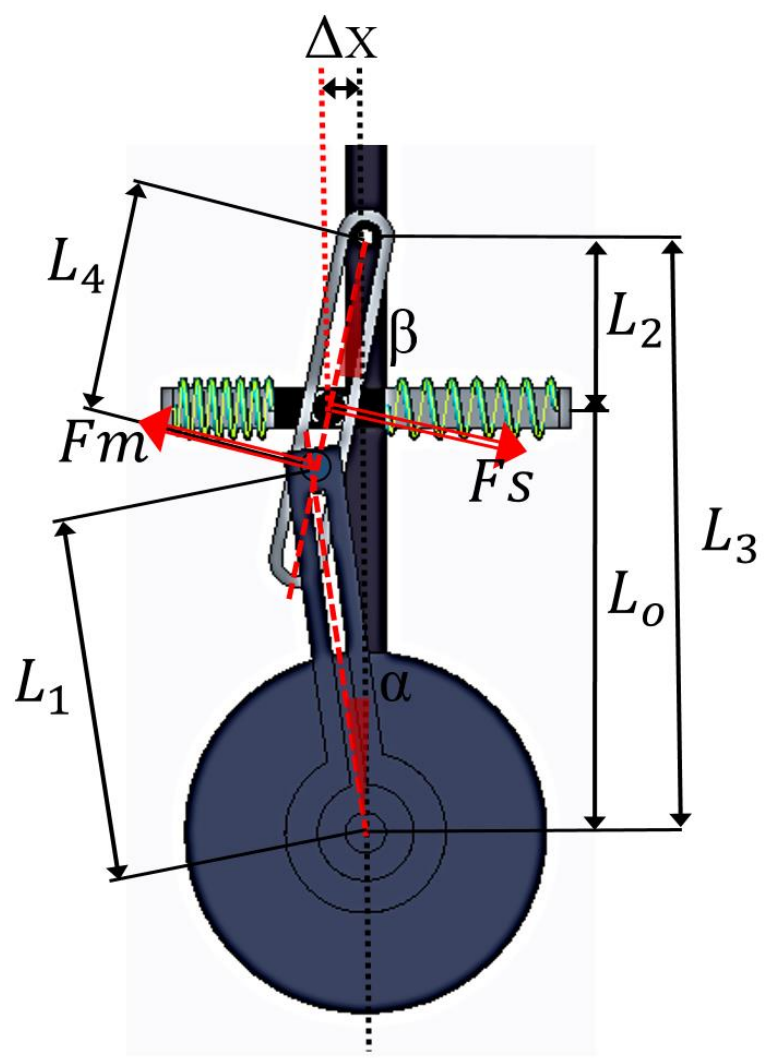

Figure 6-5. ARES-XL, Diagram for Torque Calculations. 
Based on Figure 6-5, the relationship for a torque measurement, as a function of $\mathrm{L}_{2}$ and the compression $\Delta \boldsymbol{X}$, can be obtained:

The distances $\mathrm{L}_{3}$ and $\mathrm{L}_{1}$, are fixed values that can affect the size of the system, the range of stiffness and the maximum deflection, $\alpha$, allowed at the joint.

$$
\tau=F m \times L_{1}
$$

In Eq. 6-1, the basic relationship for the torque at the joint is given. The projection of $\mathrm{Fm}_{\mathrm{m}}$ perpendicular to the slotted bar-B2, is shown in Figure 6-5. With the known and measured values, $\mathrm{L}_{1}, \mathrm{~L}_{2}, \mathrm{~L}_{3}$, and $\Delta \boldsymbol{X}$, the rest of the variables can be obtained.

- The angle $\beta$, created between the slotted bar -B2 and the line passing through the axis of rotation of the joint and the point of rotation of the slotted bar-B2, is known from the relationship:

$$
\beta=\operatorname{atan} \frac{\Delta X}{L_{2}}
$$

- The effective arm length $\boldsymbol{L}_{\mathbf{4}}$, that defines the distance between the coupling point and the pivot axis of the slotted bar $-\mathrm{B} 2$, is obtained with the second order relationship shown in Eq. 6-3.

$$
\boldsymbol{L}_{\mathbf{4}}{ }^{2}-\left(2 \cdot L_{3} \cdot \cos \boldsymbol{\beta}\right) \cdot \boldsymbol{L}_{\mathbf{4}}+{L_{3}}^{2}-L_{1}{ }^{2}=0
$$

- $\quad$ The deflection $\alpha$, directly related to $\beta$ is given by the relationship Eq. 6-4.

$$
\alpha=L_{4} \cdot \frac{\operatorname{asin}(\sin \beta)}{L_{1}}
$$

- The contact of the inner face of the slotted bar-B2 with the Pivot-P1 is always tangent. The force $F S$, transmitted through the slotted bar will be orthogonal to - 
B2 and the angle between the springs axis and the force equal $\beta$. Hence, knowing the springs properties and the compression $\Delta \boldsymbol{X}$ for identical elastic elements,

$$
F S=\frac{2 . \Delta X . K}{\cos (\beta)}
$$

The following relationship is obtained by using the previous equations. The torque at the joint taking advantage of the compliance of the actuator can be calculated using the expression,

$$
\tau_{\text {joint }}=\frac{2 \cdot \Delta X \cdot K}{\cos \alpha \cdot \cos (\beta)} \cdot \frac{L_{2} \cdot L_{1}}{L_{4}}
$$

Where,

$\Delta \boldsymbol{X}$, correspond to the springs' compression, measured by the linear encoder.

$\boldsymbol{\alpha}$, and $\boldsymbol{\beta}$ the bar $-\mathrm{B} 2$ and joint deflection due to the springs compression.

$\boldsymbol{K}$, is the equivalent rigidity of the elastic elements in the slider device.

$\boldsymbol{L}_{2}$, is the distance that can be adjusted by M2, between the joint axis and the slider device.

$\boldsymbol{L}_{\mathbf{4}}$, effective arm length between the pivot in the slotted bar -B2 and the coupling point.

$\boldsymbol{L}_{\mathbf{1}}$, fixed distance, from the joint axis to the pivot $-\mathrm{P} 1$ in the coupling point.

The lengths relationship $\frac{L_{2} \cdot L_{1}}{L_{4}}$ observed in the Eq. 6-6, evidences that the system can significantly vary the range of stiffness by adjusting the position of the elastic elements. If the distance $\boldsymbol{L}_{\mathbf{2}}$ is set to zero, the joint won't oppose resistance to a torque exerted at the joint. Similar values to $\boldsymbol{L}_{\mathbf{4}}$ will make the system behave similar to the ARES actuator, and $\boldsymbol{L}_{\mathbf{2}}>\boldsymbol{L}_{\mathbf{4}}$ will produce higher values of stiffness compared to the previous version. In Figure 6-6 the range of torques and stiffness for the design parameters are shown. The maximum deflection achievable by this system is higher than $20 \mathrm{deg}$, at zero stiffness, which can be used to emulate swing at the knee. 


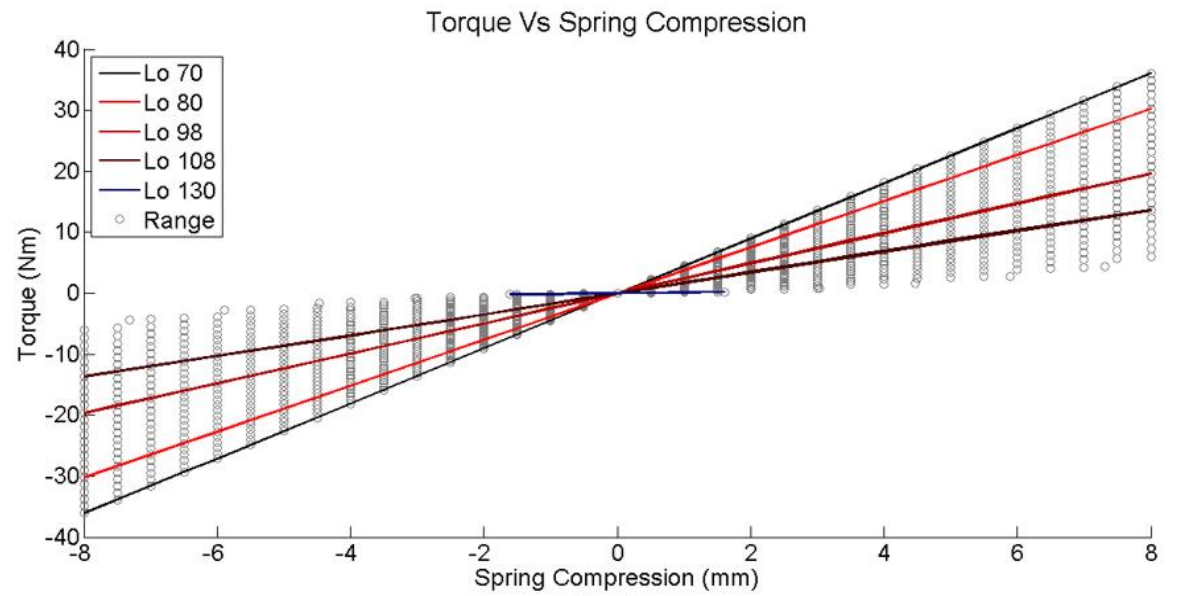

(a)

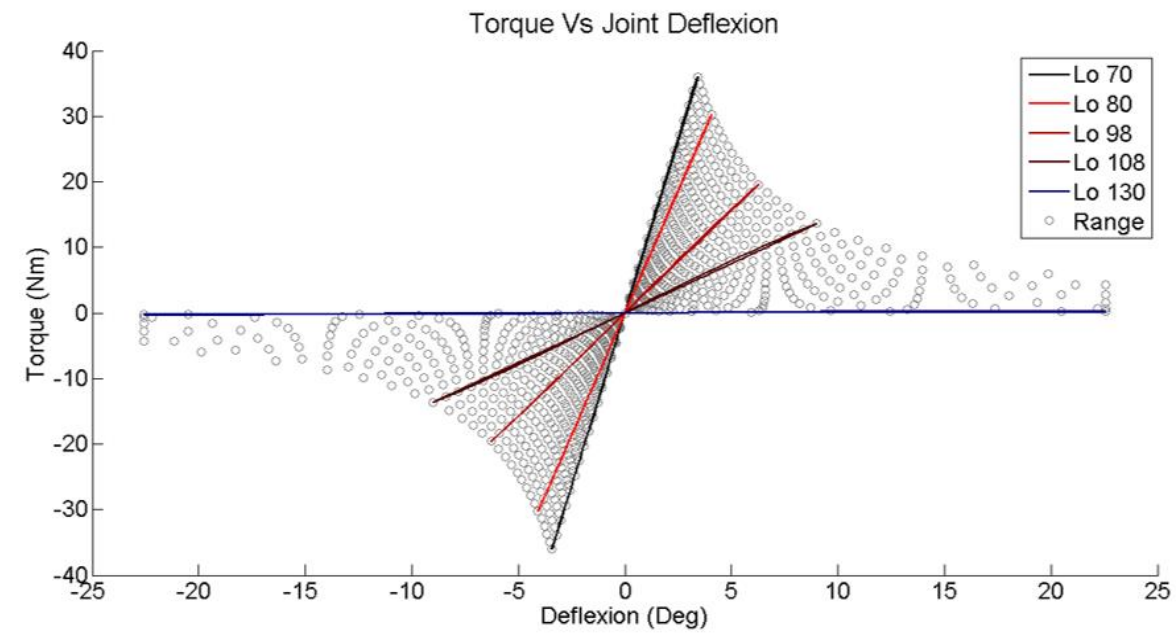

(b)

Figure 6-6. ARES-XL Range of Stiffness. a) Torque Range for Different Positions of Lo b) Stiffness Range for Different Positions of Lo.

\subsubsection{Simulation-Based Force Sensor Evaluation}

To evaluate the proposed working principle, simulations were performed with the help of CREO Parametric, a Mechanism-CAD Software. A simplified model, containing the main 
elements of the actuator was introduced into the software. The parameters considered in the simulations are listed below,

- Mass properties and values were kept close to those in the real prototype.

- The coupling between the stiff complex and the compliant mechanism, as well as the pivot $-\mathrm{P} 2$ and the slotted bar $-\mathrm{B} 2$, were modeled as a contact point.

- In order to measure torques up to $40 \mathrm{Nm}$, springs pre-compressed $10 \mathrm{~mm}$ with an approximate of $\mathrm{K}=16.8 \frac{\mathrm{N}}{\mathrm{mm}}$ were placed into the simulation.

- The springs are constrained to linear guides to avoid bending.

Simulations of the dynamic behavior were commanded applying different torque patterns. Several parameter were continuously measured during simulations. ARES-XL incorporates a set of sensors capable of tracking the joint position, the motor-M1 turns, the length of the arm $\boldsymbol{L}_{2}$, and the elastic elements compression. Using the same known variables from the real prototype and by implementing the equations from the previous segment the mechanism is tested in the simulations in order to evaluate the torque measuring capability.

Figure 6-7 shows the result of the simulation after applying a sine torque profile with 4 different amplitudes. In order to evaluate the system, the position of the slider was adjusted by decreasing the length $\boldsymbol{L}_{\mathbf{0}}$, distance from the axis of rotation of the joint and the slider with the

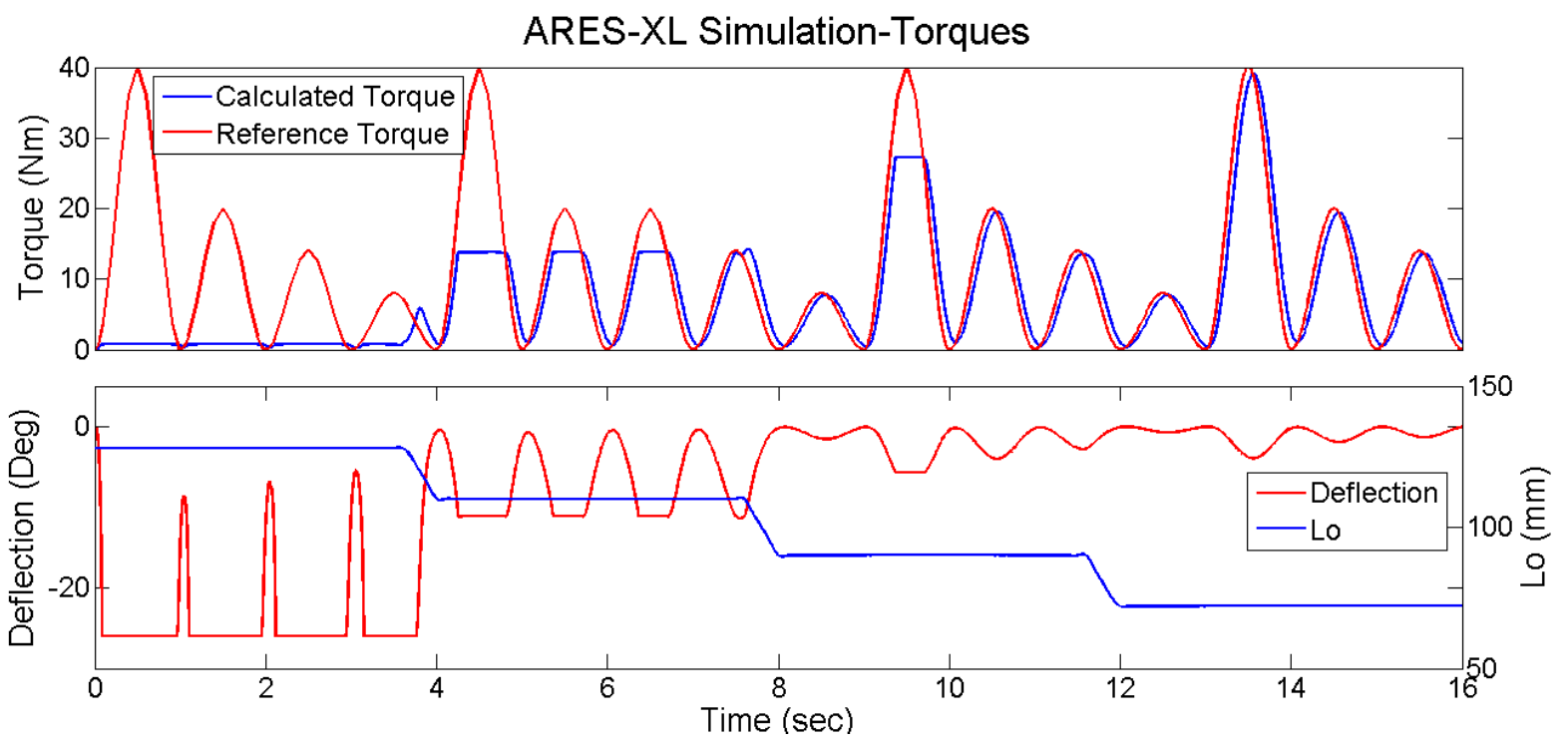

Figure 6-7. Open-Loop Torque Tracking Simulation with Different Sine Torques. 
elastic elements in the compliant mechanism. By modifying the distance, the stiffness of actuator is adjusted and as so, the range of deflection, and the maximum torque possible to measured. At minimum stiffness, the system does not oppose to the load at the joint, so the system reaches its maximum deflection even at low torques exerted. The maximum deflection of the system is approximately 25 degrees. The torque tracking in open loop fits to the commanded, but quick saturation can be observed at higher values of $\boldsymbol{L}_{\mathbf{0}}$. As the compliance of the device is tight to the embedded force sensor range, this saturation is expected. A proper adjustment of the stiffness needs to be made in order to achieve good torque tracking capability.

Figure 6-8 presents the results of the simulation after applying step- and ramp loads at the joints. The figures illustrate that the maximum deflection at the joint is also related to the stiffness level. In the simulation it is possible to appreciate 3 different maximum deflections, also associated with saturated torque measurements. In Figure 6-6, this behavior can also be spotted. The elastic elements used to provide the compliant behavior to the joint, can be only compressed to a certain limit. When a torque at the joint causes the elastic elements to be at its maximum compression, the system becomes rigid in that direction. The proposed actuator is intended to be torque controlled, and will try to exploit the natural dynamics of the legs during walking. A proper, and fast adjustment of the compliance of the actuator will allow the trade off to detect small torques, large torques, and allow the required compliance in the performed task.

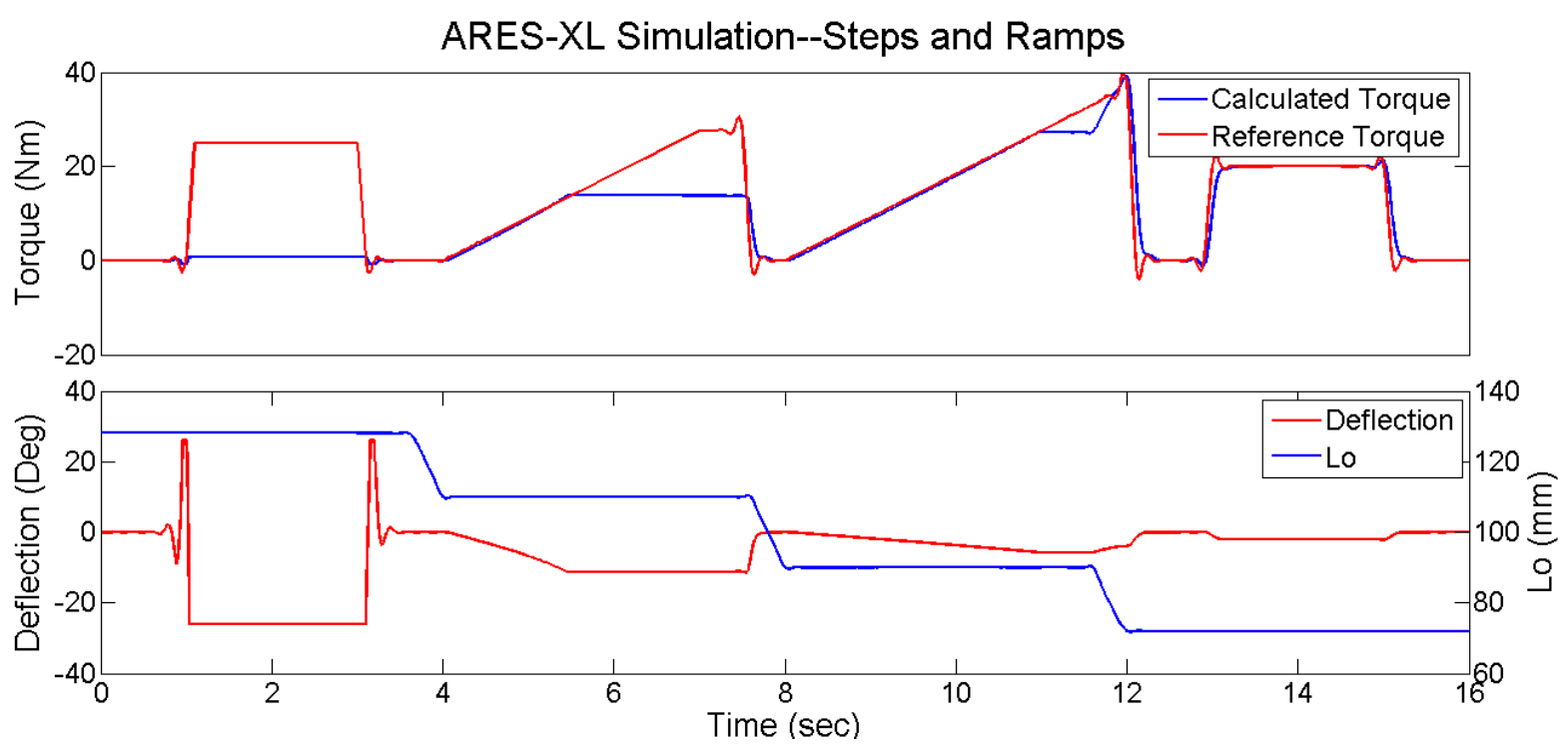

Figure 6-8. Ramps and Steps Torque- Simulation in ARES-XL 


\subsection{ARES-XL Prototype}

Variable Stiffness Actuators are commonly designed for particular applications, ARES-XL prototype is intended to be incorporated into multiple joints in the ATLAS exoskeleton. For this reason this actuator intends to present the required versatility in order to adjust the actuator to the joint where it is implemented. Following the mechanical design proposed in this chapter a prototype of the compliant joint, ARES-XL, was built. Most of the prototype is made of aluminum to reduce weight, the encoders housings were made with a 3D printer as well as the Locking components for test proving the locking mechanism.

Figure 6-9 shows the main views of the ARES-XL CAD-design with its main dimension. The discussed actuator is incorporated into an articulated joint, comprising an upper limb, and a lower limb. The basic mechanical properties of the compliant system can be seen in

Table 6-1, where the maximum deflection as well as the minimum stiffness that the mechanism can reach, are preliminary evidence that this new system highly exceed its predecessor.

Table 6-1. ARES-XL and ARES Mechanical Properties

\begin{tabular}{lll}
\hline & ARES-XL Properties & ARES Properties \\
\hline Peak Torque & Up to $76 \mathrm{Nm}$ & Up to $76 \mathrm{Nm}$ \\
Max Deflection & $\pm 23^{\circ}$ & $\pm 8^{\circ}$ \\
Range of Stiffness & $0-15 \mathrm{Nm} / \mathrm{deg}$ & $2.5-13 \mathrm{Nm} / \mathrm{deg}$ \\
Stiffness Adjusting & $0.25 \mathrm{sec}$ & $0.25 \mathrm{sec}$ \\
time (Teo) & $1.3 \mathrm{Kg}$ & $1.2 \mathrm{Kg}$ \\
Weight & $260 \mathrm{~mm}$ & $260 \mathrm{~mm}$ \\
Length & $80 \mathrm{~mm}$ & $80 \mathrm{~mm}$ \\
Width & $90 \mathrm{~W}$ & $90 \mathrm{~W}$ \\
Power &
\end{tabular}



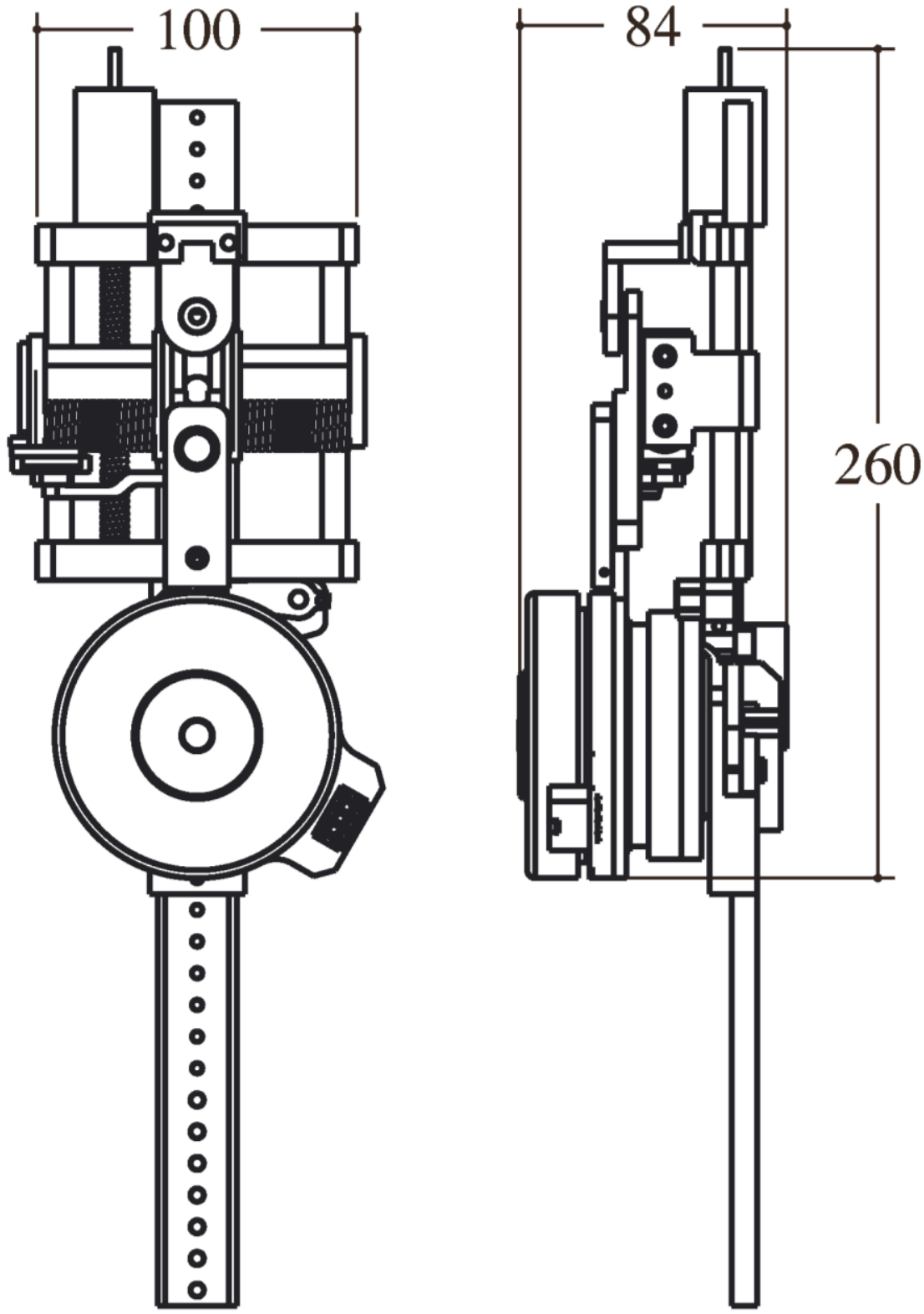

Figure 6-9. ARES-XL Joint- CAD Model with Main Dimension. 


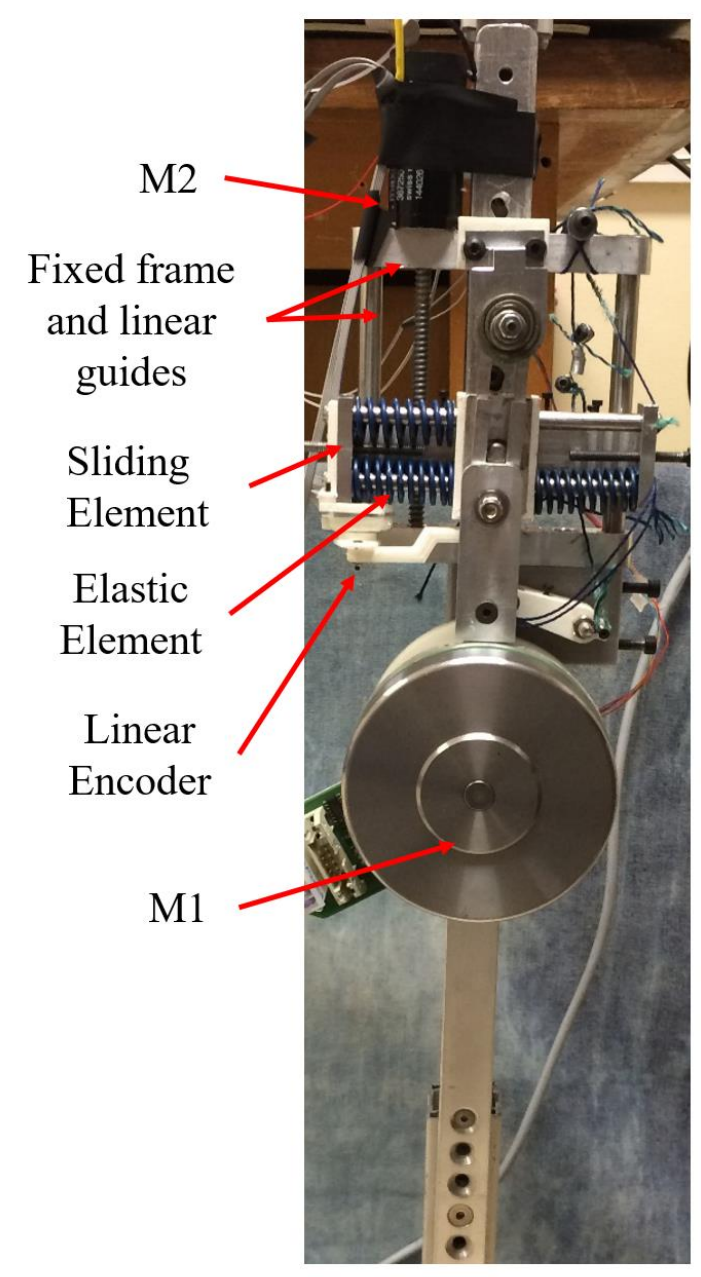

(a)

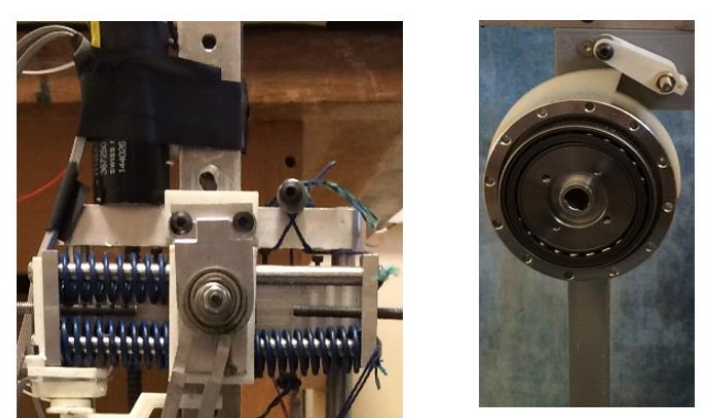

(c)

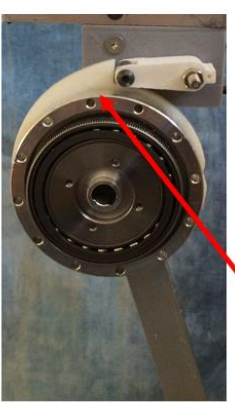

(d)
Round

Element with

locking tooth

-RT

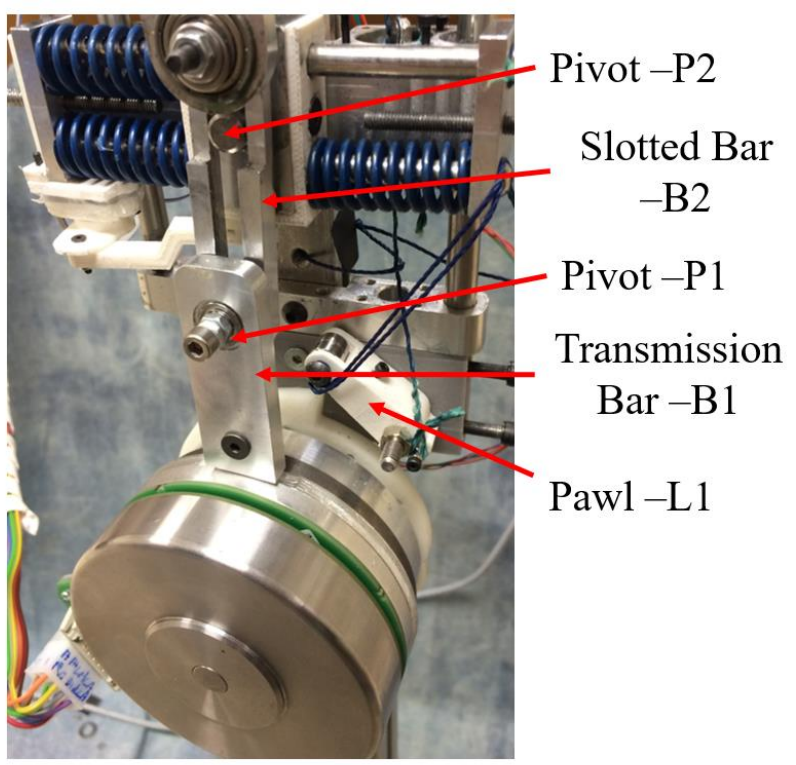

(e)

Figure 6-10. ARES-XL Prototype.a) ARES-XL Prototype-Front View. Pawl -L1 not Being Pulled. b) ARES-XL Prototype-Front View. Pawl -L1 Being Pulled and Stiffness Near Zero. c) View of the Locking System-Ratched with Pawl Disengaged. d) View of the Locking System-Ratched with Pawl and Locking Engaged. e) ARES-XL Prototype -Custom View. Coupling Elements Highlighed. 
The actuator prototype presented on Figure 6-10, is an improvement of the previous realization ARES, and has been built and assembled with the following considerations.

- The Pivot -P2, see Figure 6-10 (e), presents a cylindrical shape to facilitate tangential contact between the slotted bar $-\mathrm{B} 2$ and the pivot $-\mathrm{P} 2$, thus the transmitted force to the pivot $-\mathrm{P} 2$ is near to a radial force direction. The pivot $-\mathrm{P} 2$ is made of steel as all the forces are transmitted to it. To reduce the friction when adjusting the stiffness, the pivot -P2 can roll along the inner face of the slotted bar $-\mathrm{B} 2$ by means of a set of radial bearings.

- $\quad$ Pivot $-\mathrm{P} 1$, similar to Pivot -P2, presents a cylindrical shape with the capability to spin in the transmission bar $-\mathrm{B} 1$ by means of a set of radial bearings.

- The Slotted bar -B2 is a slender bar made of aluminum 7075. The Pivot -P1, and pivot $-\mathrm{P} 2$ are in contact with the inner face of the slotted bar $-\mathrm{B} 2$. The thickness of the bar is enough to avoid both pivots to collide when rolling inside the slotted bar $-\mathrm{B} 2$.

- Linear guides are placed in the sliding element, shown in Figure 6-10 (a), to guide the elastic elements and avoid bending that can affect the linear compression used for torque calculations.

- Two calibrated steel rods are used as linear guides in the compliant mechanism. Connected to the fixed frame, they transfer the reactions forces to the upper link of the joint. There is no lateral stress expected at the non-backdriveable Ball-Screw.

- The locking of the joint is achieved by the ratchet mechanism. In Figure 6-10 c) and d) it can be seen that when a certain deflection is reached the pawl-L1 gets to a singular position that if pressed by the tooth $-\mathrm{RT}$ the deflection in that direction 
is constrained. To disengage the locking, the pawl needs to be pulled by the cable connected to the sliding element, see the tensioned cable in Figure 6-10 b). The end of the pawl $-\mathrm{L} 1$ in contact to the tooth $-\mathrm{RL}$ can spin to facility the unlocking when the cable pulls.

\subsubsection{ARES-XL Assessment}

To evaluate the behavior of the actuator several experiments were performed focused on testing the main perks of the compliant system. The experimental setup used, consisted on a couple of articulated links resembling a joint from a robotic exoskeleton. Attached to the lower link, a link extension holding an adjustable load was set, see Figure 6-11. Implementing the same electronics and control from the previous realization, online kinematic data and current consumptions were logged for offline data analysis. By designing and implementing an appropriate joint trajectory, relevant capabilities of ARES-XL can be tested on the test bench.
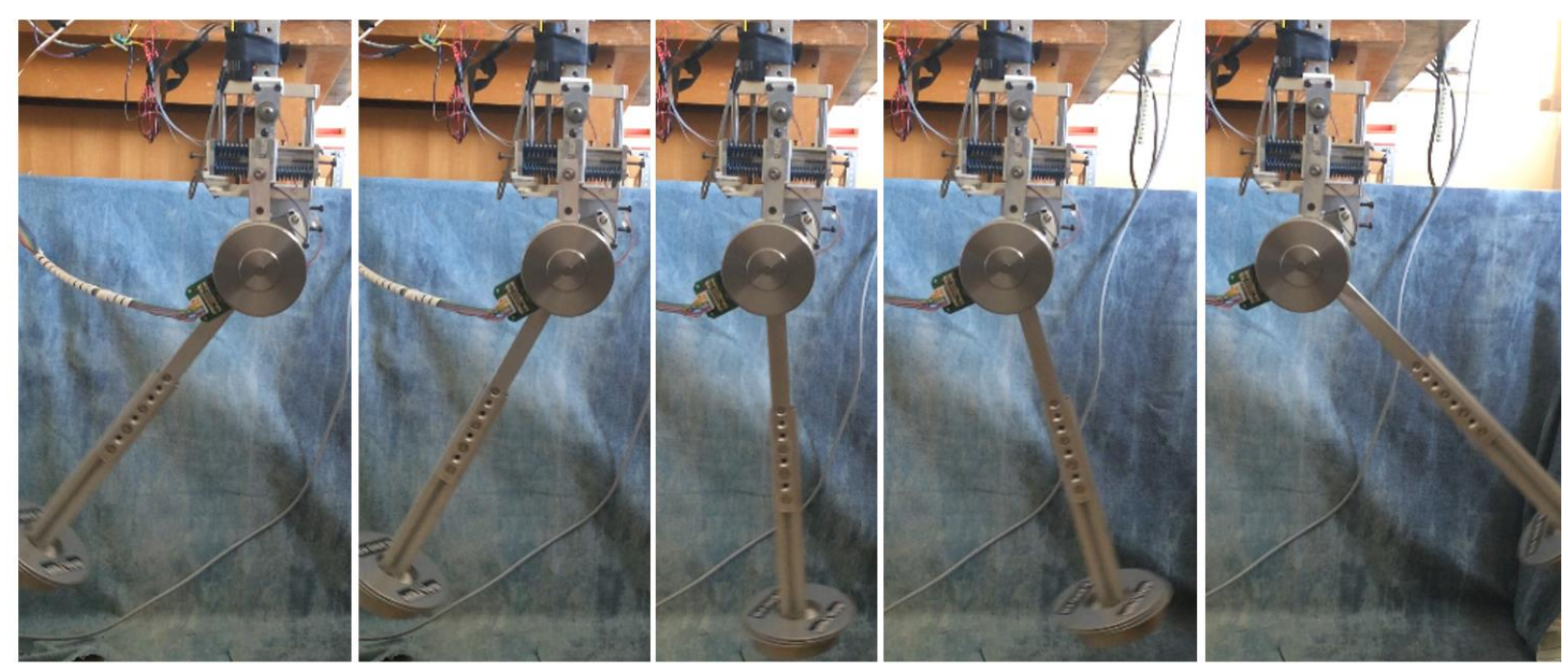

Figure 6-11. ARES-XL Bench Test Snaps. 


\subsubsection{Embedded Torque Measurement Validation}

Torque tracking during operation is one of the main functionalities of the compliant joint. In order to validate the embedded force sensor in the prototype, a sinusoidal trajectory was commanded to the joint; where by adjusting the load in the lower link, a quasi-sinusoidal torque will result as a load exerted at the joint.

The results obtained for two different load weights are shown in this sub-section. A load equivalent to $1.6 \mathrm{~kg}$ attached to the lower link at a distance of $354 \mathrm{~mm}$ from the joint axis was used to test the joint at low torques. Figure 6-12 presents the theoretical torque at the joint, and the calculated torque computed using the relationship given by Eq. 6-6 and the compression of the elastic elements during the tests. Figure 6-12 a) presents the result with the stiffness of the joint fixed to a Low stiffness value, while in Figure 6-12 b) the stiffness was fixed to a higher value.

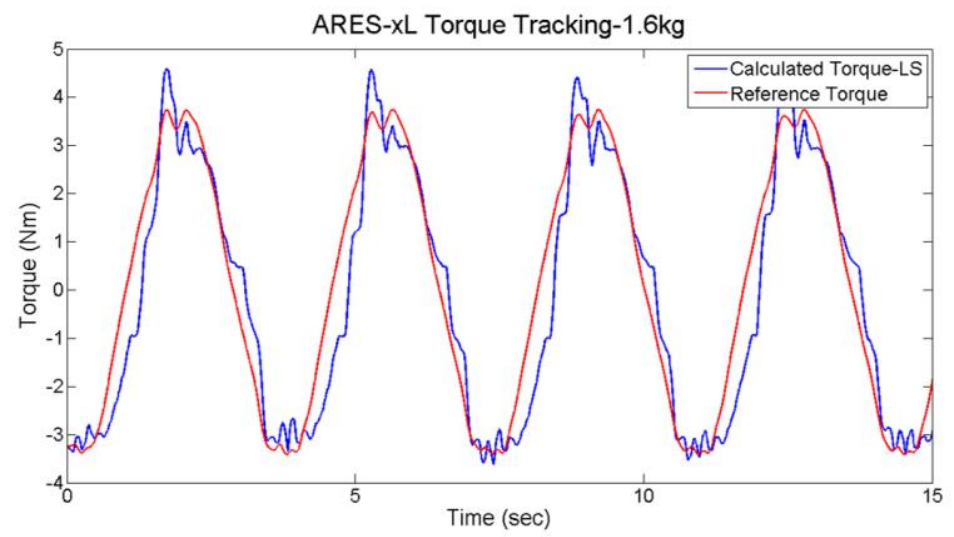

(a)

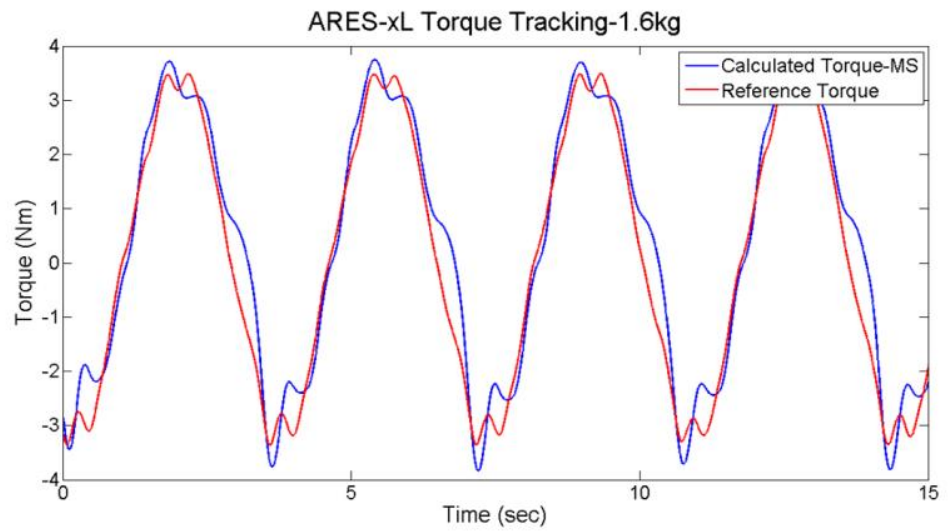

(b)

Figure 6-12. Torque Tracking in ARES-XL at 2 Levels of Stiffness and 1.6Kg Load. 
The theoretical and measured values present a very good fit, and from the figures it is possible to notice that at lower stiffness the delay given by the elastic elements is greater than at medium stiffness. However at the peaks, where the inertia of the load generates peaks, lower stiffness configuration settles closer to the load value, opposed to medium stiffness where the system behaves damped, and the load peak looks as an overshot and settles about $1 \mathrm{Nm}$ from the theoretical reference. Similar to ARES, this improved version presents tight relationship between stiffness and torque resolution.

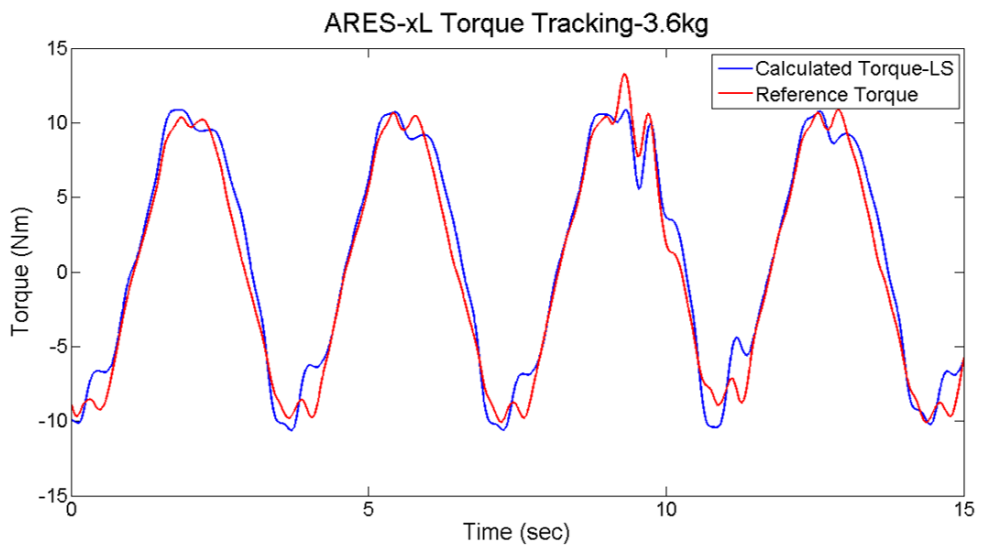

(a)

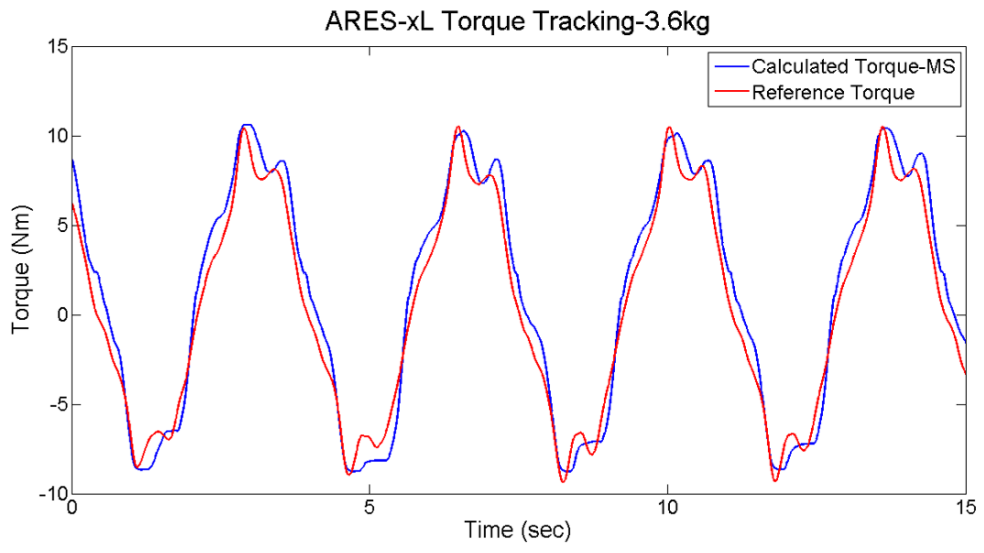

(b)

Figure 6-13. Torque Tracking in ARES-XL at 2 Levels of Stiffness and 3.6Kg Load.

Figure 6-13 shows the results after adjusting the load to $3.6 \mathrm{~kg}$ and an equivalent link arm of $3.6 \mathrm{~mm}$. Torques up to $\pm 10 \mathrm{Nm}$ were tested in this configuration at 2 different stiffness levels. The torque tracking fits closely to the theoretical torque, both stiffness levels seem to be suitable for the given load range. Low stiffness will allow larger deflections while still maintaining a good 
torque sensing capability, this can be an advantage when the actuator is intended to be used for multiple purposes.

\subsubsection{Ankle Emulation}

The ankle joint during gait generates positive and negative power as discussed in chapter 3. During almost all the support phase this joint is capable of behaving as a passive mechanism, by adapting the joint position as a consequence of the foot loaded with the body weight and the ground contact. However, it is commonly discussed in the design of prosthetic devices and gait analysis, the limitations of conventional electric motors to supply the necessary power for the push off at the end of the support phase. ARES-XL is proposed as a suitable actuation system at the ankle; by implementing a stiffness adjustment during the locomotion cycle, in combination with the power generation of the main motor-M1, ARES-XL is capable of providing a higher torque than a conventional stiff motor.

To validate ARES-XL as a suitable actuation system for the ankle joint, a combination of a state machine and the implementation of specially designed trajectory were implemented in the experiment. Three particular cases were tested,

- $\quad$ Fixing the stiffness of the joint at medium stiffness (-MR).

- Blocking the compliance by mechanically constraining the elastic element in the compliant element (-R) and making it work as a stiff actuator.

- Adjusting the stiffness during operation to exploit the dynamics of the system by implementing a state machine in the compliant mechanism (-AR), see Figure 6-14.

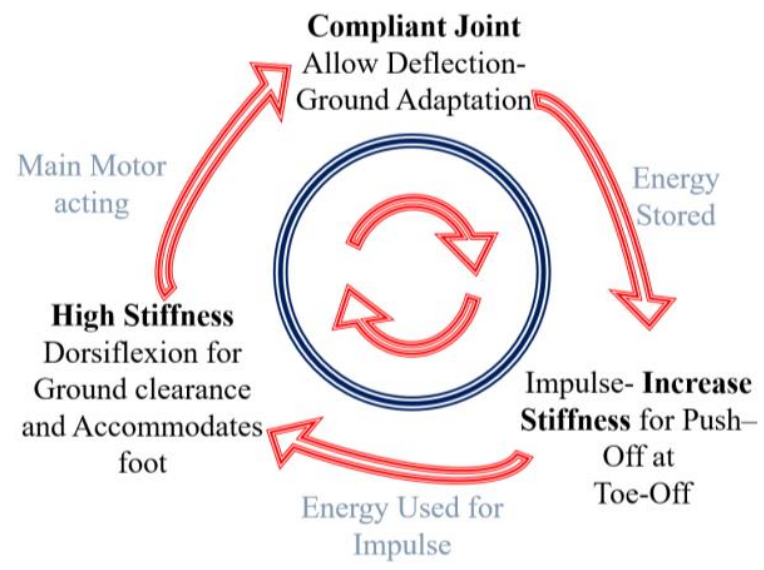

Figure 6-14. State-Machine at the Joint to Emulate the Ankle. 


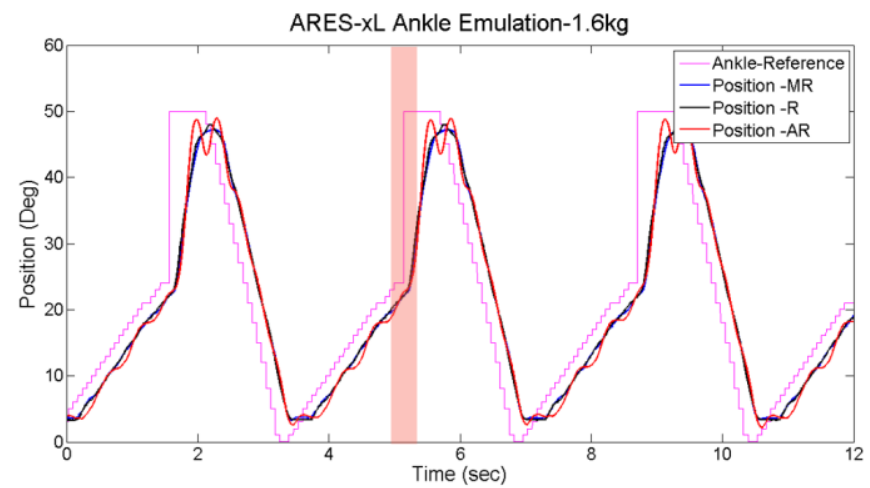

(a)

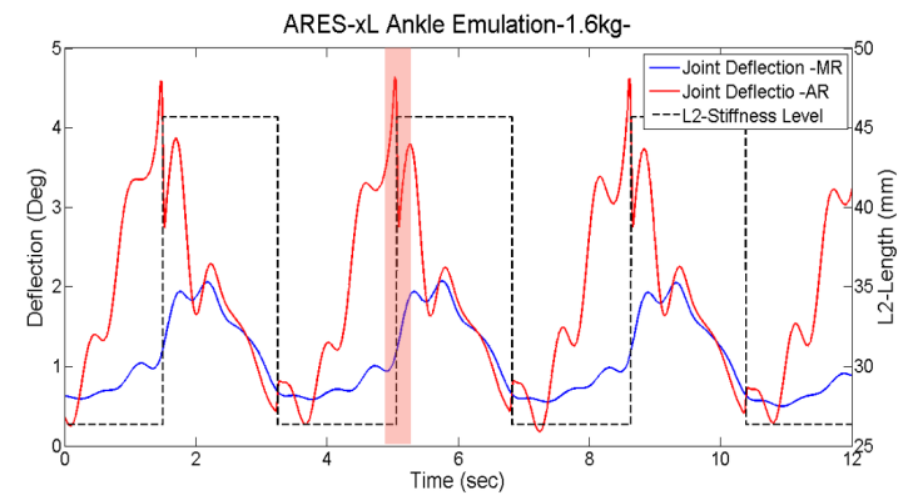

(b)

Figure 6-15. Ankle Emulation. a) ARES-XL Position Response. b) Stiffness Levels -State Machine

The Figure 6-15 a) shows the implemented trajectory. During the first part of the motion, the joint load will gradually increase. This section of the trajectory emulates the support phase and it is followed by a step increment of the joint position. The actuator response won't be able to deliver an instant motion, similar to the case of the push-up at the ankle. The last section of the trajectory does not require high power, neither high speed, the ankle at the swing re-accommodates to continue the gait cycle, and assist the ground clearance.

The implemented state machine from Figure 6-14 can be seen in Figure 6-15 b). The stiffness will be adjusted between two different levels. Low stiffness during the emulation of the support phase, followed by high stiffness at the time that the step is commanded at the trajectory. The joint position for the tested cases are shown in Figure 6-15 a), the case $-\mathrm{R}$ is used as a comparison with a stiff actuation configuration, and as consequence Figure 6-15 b) does not show deflection at the joint for this configuration. The -AR shows better fit from the joint following the 
step trajectory and the reached peak higher than the other two cases. Position is related to the torque at the joint, thus -AR seems to achieve slightly higher torque.

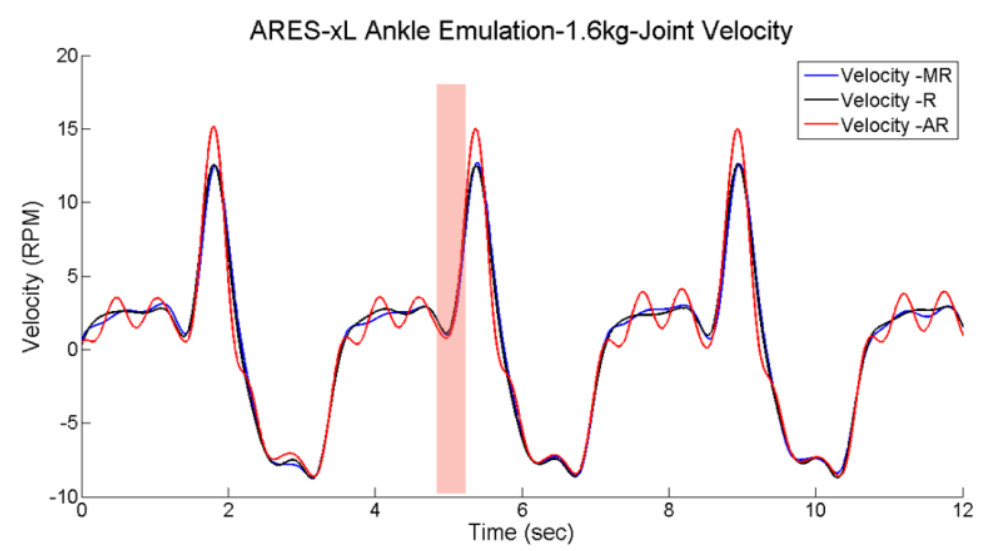

(a)

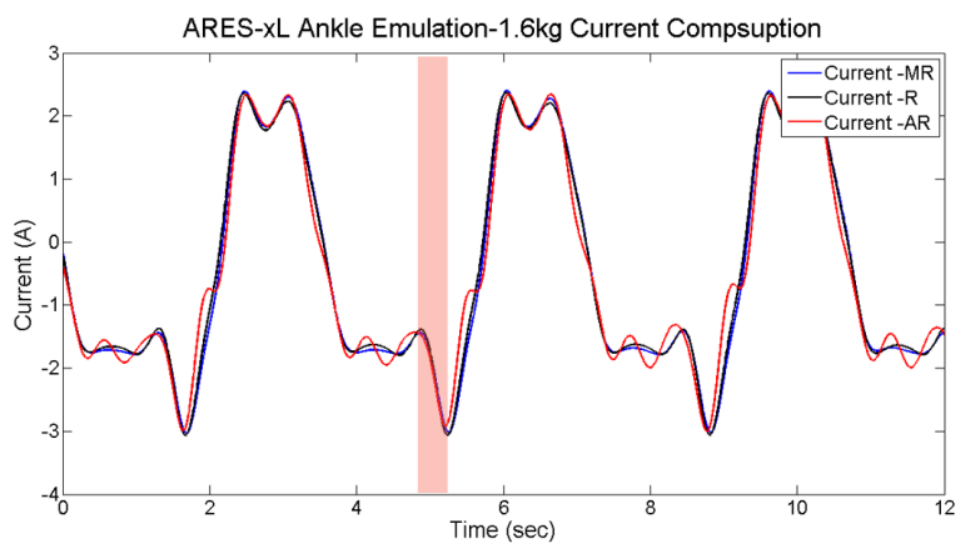

(b)

Figure 6-16. Ankle Emulation. a) ARES-XL Joint Velocity. b) Current Consumption.

During the support phase, -AR and -MR present deflection at the joint, this deflection can be translated into energy stored at the joint when used as a passive joint. The stiffness at -AR was set lower than $-\mathrm{MR}$, thus at the same load more deflection can be seen at -AR. At the push-off the power at the joint changes, with the bench test this condition can-not be replicated. However it can be shown that besides the energy stored at the elastic elements, by adjusting the stiffness of the system, an impulse can be given to the joint. The deflection is a function of the elastic elements compression, and the joint's stiffness. When the stiffness is increased the joint's deflection 
immediately decreases, the energy is translated directly to the joint and can be evidenced in Figure 6-16 a), by the significant higher velocity reached by -AR compared to the other two cases.

During a dynamic locomotion cycle, the energy stored at the joint during the support phase can be used to reduce the power required by the main motor-M1; by adjusting the stiffness level during the cycle, allowing the joint to store a larger amount of energy, and increasing the stiffness at the push-off, the deflection of the joint can contribute to a larger energy given to the joint, and as a consequence even less power required by the main motor. Figure 6-16 b), shows the current consumption for the tested cases, evidencing no significant differences in the main motor $-\mathrm{M} 1$ consumption while achieving better step response and higher velocity at the joint with $-\mathrm{AR}$.

The large deflection allowed by ARES-XL could be used successfully to store energy during gait at loading response in the ankle joint. By dynamically adjusting the stiffness at this joint just before the toe-off, an impulsive reaction due to the previous deflection of the system, combined with the now stiffer configuration is produced at the ankle joint. The power delivered at the ankle, taking advantage of the biomechanics of the gait, stiffness control, and the mechanical properties of ARES-XL exceeds the short-time power that can be achieved with a stiff configuration, or a simple compliant joint.

\subsubsection{Knee Swing Emulation.}

In order to implement ARES-XL at the knee joint, the actuator needs to be able to behave as a compliant joint with large deflection, hold the energy stored and released when commanded, and be capable of generating power when required. All those requirements were discussed in chapter 3, where after analyzing the knee biomechanics the following conditions need to be accomplished by the joint:

- Compliant behavior during loading response. Absorb energy by accepting the body weight, flexing the knee close to 10 degrees, followed by extending the knee using the stored power during the flexion.

- Pre-Swing flexion. Just before toe-off the knee flexes over 10 degrees. The energy absorbed should be transferred to another joint or stored for later phases.

- Initial-Swing. The knee generates power to continue the flexion to achieve a proper ground clearance. 
- Final-Swing. The knee flexed about 60 degrees, extends to accommodate the leg for the heel strike. The swing is ideally a combination of free joint and power generation to accommodate the right extension.

From the ankle emulation experiments, and the intrinsic compliance of the system, ARESXL is capable of providing the behavior during loading response. By setting the stiffness to a proper value based on the weight of the subject, flexion at the knee absorbing energy, followed by the knee extension by decompression of the elastic elements when the load decrease, this can be achieved with ARES-XL and its large range of deflection.

At the pre-swing the stored energy will quickly try to release itself as soon as the leg leaves the ground, deviating the joint from its trajectory. This can translate on the main motor-M1 working extra to compensate the deviation of the joint from the commanded path. Implementing the add-on locking mechanism, the deflection at the pre-swing can be locked to continue to the next phase, where the direction on the load changes while maintaining the same direction of rotation. The energy stored is not wasted nor opposing the natural motion. The energy supplied to flex the knee while the locking is engaged is used solely to produce the flection.

Finally, at the end of the swing, the stiffness of the system is set to a low value. Disengaging at the same time the locking mechanism, allowing a free swing without energy of at least the amount of deflection reached at the pre-swing.

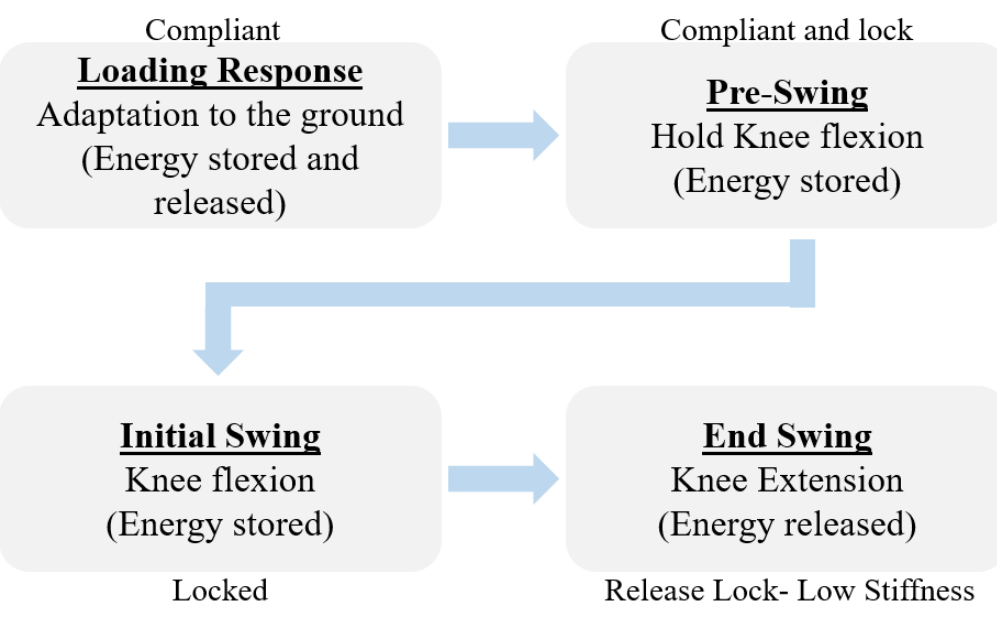

Figure 6-17. Diagram of the Knee Behavior. 
In order to validate ARES-XL as an actuation system capable of storing the energy at preswing and present a free swing motion, the trajectory presented in Figure 6-19 a) was implemented at the joint, along a state machine (See Figure 6-18) to adjust the stiffness during the cycle. The following cases were tested.

- Adjustable Stiffness to allow engaging of the locking, disengage and free swing $(-\mathrm{AR})$.

- $\quad$ Fixed stiffness at a mid value, to present a compliant joint (-MR).

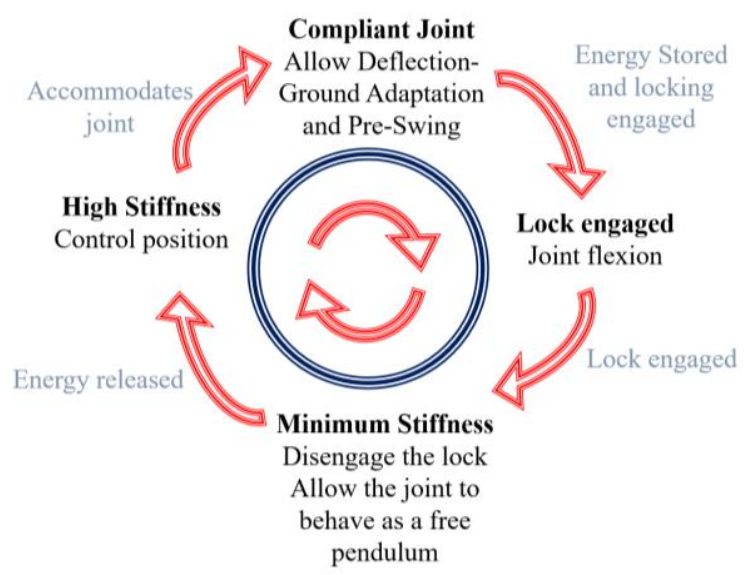

Figure 6-18. Schematics of the State-Machine Applied to Emulate the Knee Swing.

The implemented trajectory gradually increases the torque exerted at the joint, to resemble the pre-swing section of the gait. Followed by a change in the rotation direction that will end with the joint loaded in an opposite direction that in the one in the pre-swing section. Finishing with a step to zero to test the swing capability of the joint.

The test performed with fixed stiffness -MR, behaved similar to a simple sine trajectory as can be seen in Figure 6-19 c), the deflection at the joint was simply proportional to the load at the joint, as expected due to the embedded force sensor principle. From Figure 6-19 b) it can be seen that the velocity reached at the swing region, was lower for the $-\mathrm{MR}$ case.

With the implementation of a state machine for adjusting the stiffness of the system; the results for the experiment in the case -AR show that during the pre-swing section the deflection was larger than 10 degrees with a low stiffness value. This large deflection in the desired direction, 


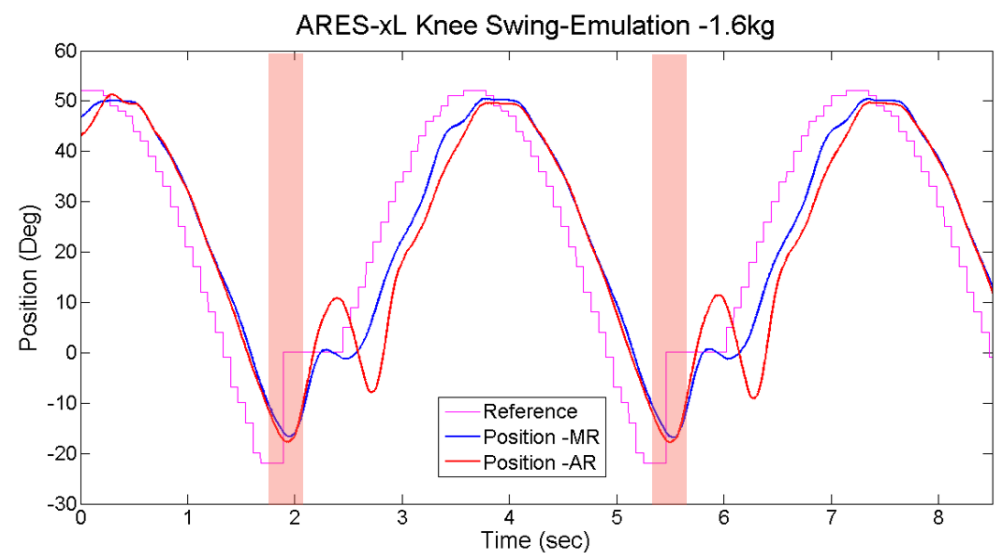

(a)

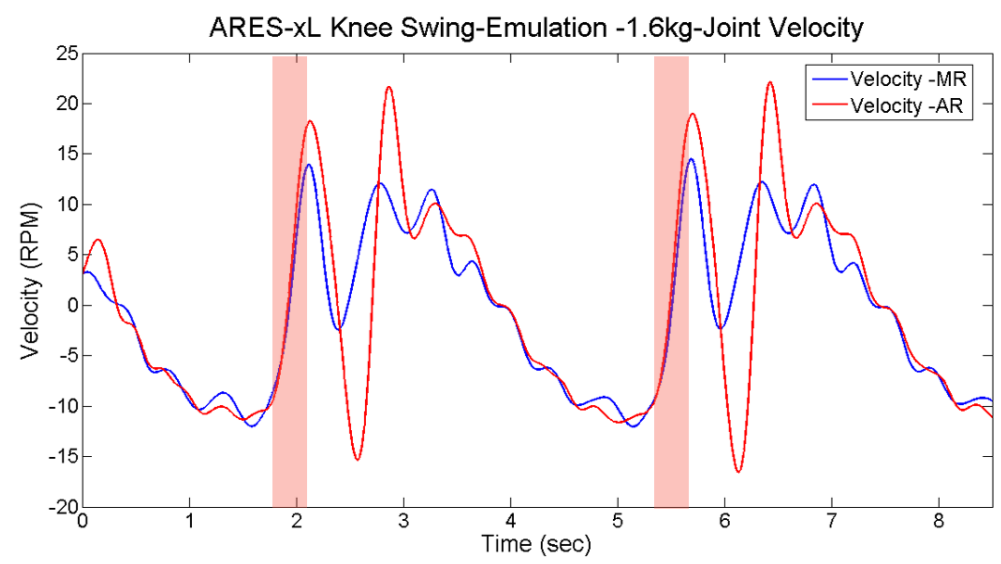

(b)

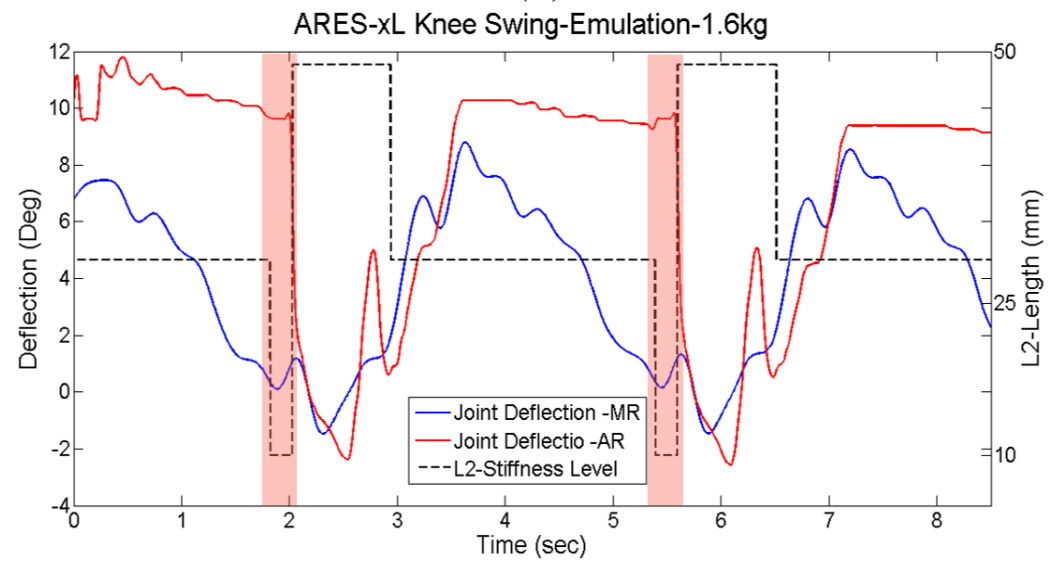

(c)

Figure 6-19. Knee-Swing Emulation. a) ARES-XL Joint Position and Trajectory. b) ARES-XL Velocity. c) ARESXL Deflection and Stiffness Levels State Machine.

caused the locking to engage as can be appreciated in Figure 6-19 c) by the constant deflection just before the swing region. With the deflection locked, the joint continued following the desired 
trajectory until the stiffness of the system was set to a minimum value. The stiffness adjustment tensioned the cable attached to the pawl $-\mathrm{L} 1$, forcing the locking to disengage. At this point the stiffness of the system is near minimum with deflection reached at the pre-swing larger than 10 degrees. Due to the potential energy, the joint deflection drops to zero as shown in Figure 6-19 c). Similar to the swing in the human locomotion, the main motor does not require to supply power to achieve this motion. The joint moves freely due to the potential energy and the stored energy at the compliant mechanism. Figure 6-19 b) shows how the velocity reached with -AR is higher than the delivered with a simple compliant actuator. This velocity is directly related to the inertia of the link and its potential energy, thus implemented in a mechanism to achieve the swing of the knee, should result in a more natural and efficient movement.
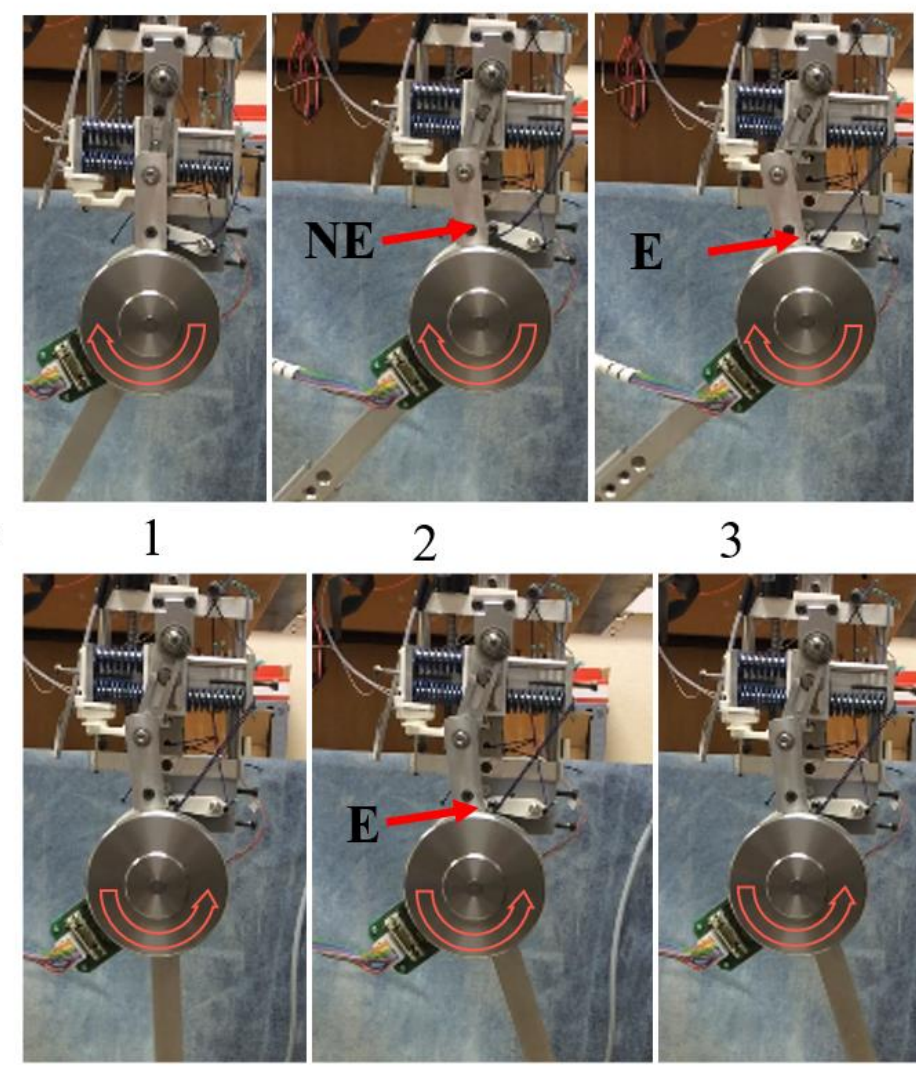

6

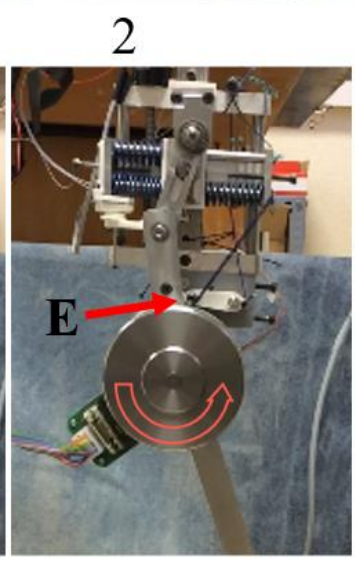

7

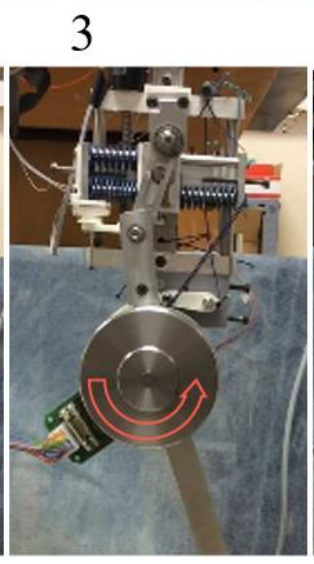

8
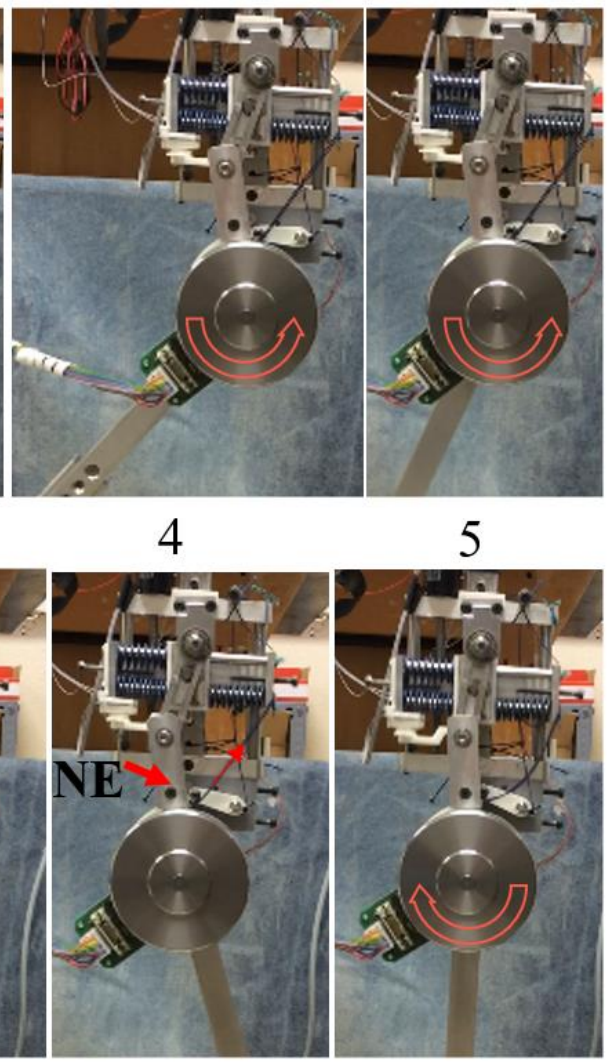

9

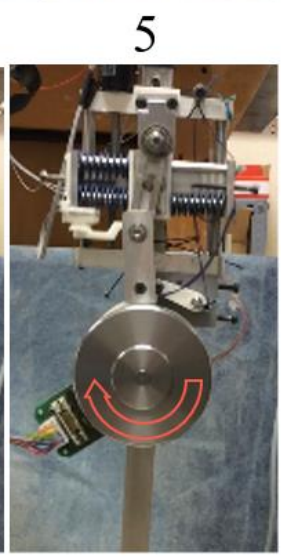

10

Figure 6-20. ARES-XL Locking and Unlocking Sequence. Locking: Not engaged (NE), and Engaged (E). 
ARES-XL could be used at a knee joint, to exploit its intrinsic compliance during the loading response, followed by accepting the weight of the body at the pre-swing and storing this energy for its release at the end of the swing. During the stages where the lock is engaged, the system was able to follow the correct trajectory without affecting the velocity profile. Figure 6-20 shows the sequence of the joint during the experiment, where the deflection of more than 10 degrees can occur without the main motor to generate power.

The developed joint presented in this chapter, outperform the original realization ARES. This novel system presents a wide range of possible uses, and based on the results from the conducted experiments, could be successfully implemented at the Knee, and Ankle joints of powered devices, such as exoskeletons or even prostheses. Table 6-2 presents a comparison between the developed systems in this $\mathrm{PhD}$ work and the most relevant systems from the review conducted in Chapter 4. ARES-XL stands out not just by its versatility, but also for the large deflection allowed, associated with possible large energy storing, and the incorporation of the novel add on locking mechanism. 
Table 6-2. Actuators Comparison Including ARES and ARES-XL

\begin{tabular}{|c|c|c|c|c|c|c|c|}
\hline Actuator/Joint & $\begin{array}{c}\text { Weight/ } \\
\text { Dimensions }\end{array}$ & $\begin{array}{c}\text { Peak/constant } \\
\text { Torque }\end{array}$ & $\begin{array}{c}\text { Stiffness } \\
\text { Range }\end{array}$ & $\begin{array}{c}\text { Stiffness } \\
\text { Adjusting time }\end{array}$ & $\begin{array}{l}\text { Stiffness Adjusted } \\
\text { by/ Max. Deflexion }\end{array}$ & $\begin{array}{c}\text { Torque } \\
\text { Measurement }\end{array}$ & Application \\
\hline Migliore`s & $\begin{array}{l}\text { Prototype, } \\
\text { big } \\
\text { dimensions }\end{array}$ & $\pm 40 \mathrm{mNm}$ & $\begin{array}{c}0,2 \text { a } 0,8 \\
\mathrm{mNm} / \mathrm{deg}\end{array}$ & $\begin{array}{c}\text { Equilibrium } \\
\text { position and } \\
\text { stiffness dependent }\end{array}$ & $\begin{array}{l}\text { Compression/ } \\
\text { Antagonic }\end{array}$ & None & $\begin{array}{l}\text { Joint/non } \\
\text { linear spring }\end{array}$ \\
\hline VSA II & $\begin{array}{c}345 \text { gr. } \\
\text { Aprox. like } \\
\text { CD-diameter }\end{array}$ & $\pm 2 \mathrm{Nm}$ & $\begin{array}{l}\approx 0 \text { a } 8 \\
\mathrm{Nm} / \mathrm{deg}\end{array}$ & $\begin{array}{c}\text { Equilibrium } \\
\text { position and } \\
\text { stiffness dependent }\end{array}$ & $\begin{array}{l}\text { Compression/ } \\
\text { Antagonic }\end{array}$ & None & $\begin{array}{l}\text { Joint/4 bar } \\
\text { linkages }\end{array}$ \\
\hline AMASC & $\begin{array}{l}\text { Aprox. } 4 \mathrm{~kg} \text {. } \\
1 \text { meter Leg } \\
\quad(\text { large })\end{array}$ & \multicolumn{3}{|c|}{$\begin{array}{l}\text { Stiffness adjusted by pretension. Slow changes at } \\
\text { low forces. Complex knee system configuration, } \\
\text { intended for running legs. }\end{array}$} & $\begin{array}{l}\text { Compression/pulley } \\
\text { System }\end{array}$ & None & Running leg \\
\hline МАССЕРА 2.0 & $2.4 \mathrm{~kg}$. & $\pm 70 / 50 \mathrm{Nm}$ & $\begin{array}{c}5-110 \\
\mathrm{Nm} / \mathrm{deg}\end{array}$ & $2.6 \mathrm{~s}$ & Compression & Embedded & $\begin{array}{l}\text { Knee Joint/ } \\
\text { rehabilitation }\end{array}$ \\
\hline VS-Joint & $2 \mathrm{~kg}$. & $\pm 180 \mathrm{Nm}$ & $\begin{array}{c}0-5.5 \\
\mathrm{Nm} / \mathrm{deg}\end{array}$ & $0.2 \mathrm{~s}$ & $\begin{array}{l}\text { Compression } \\
/ \pm 14 \text { deg }\end{array}$ & Parallel sensor & Arm Joint \\
\hline QA-Joint & $2 \mathrm{~kg}$. & $\pm 40 \mathrm{Nm}$ & $\begin{array}{l}0.4 \text { a } 9.6 \\
\mathrm{Nm} / \mathrm{deg}\end{array}$ & $0.15 \mathrm{~s}$ & $\begin{array}{l}\text { Compression } \\
/ \pm 15 \text { deg }\end{array}$ & Parallel sensor & Arm Joint \\
\hline CompAct & $1.8 \mathrm{~kg}$ & 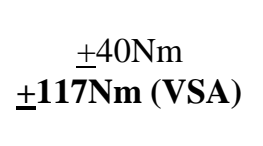 & $\begin{array}{l}9 \mathrm{Nm} \\
\text { Damping } \\
\text { 0-Rigid }\end{array}$ & $\approx 0.2 \mathrm{~s}$ & Pivot Displacement & Parallel sensor & Joint \\
\hline$A w A S-I I$ & $\begin{array}{l}1.1 \mathrm{~kg} / \mathrm{W}- \\
140 \mathrm{~mm}\end{array}$ & $\pm 80 \mathrm{Nm}$ & $\begin{array}{l}\text { Zero to } \\
\text { Rigid }\end{array}$ & $0.8 \mathrm{~s}$ & $\begin{array}{l}\text { Pivot Displacement / } \\
\pm 17 \mathrm{deg}\end{array}$ & Paralell sensor & Knee Joint \\
\hline ARES & $\begin{array}{l}1.2 \mathrm{~kg} / \mathrm{W}- \\
80 \mathrm{~mm}\end{array}$ & $\pm 76 \mathrm{Nm}$ & $\begin{array}{l}2.5 \text { a } 13 \\
\mathrm{Nm} / \mathrm{deg}\end{array}$ & $0.25 \mathrm{~s}$ & $\begin{array}{l}\text { Pivot Displacement } \\
\qquad / \pm 8 \mathrm{deg}\end{array}$ & Embedded & Multiple \\
\hline$A R E S-X L$ & $\begin{array}{l}1.3 \mathrm{~kg} / \mathrm{W}- \\
80 \mathrm{~mm}\end{array}$ & $\pm 76 \mathrm{Nm}$ & $\begin{array}{l}0 \text { a } 15 \\
\mathrm{Nm} / \mathrm{deg}\end{array}$ & $0.25 \mathrm{~s}$ & $\begin{array}{l}\text { Pivot Displacement } \\
\qquad / \pm 23 \mathrm{deg}\end{array}$ & Embedded & $\begin{array}{c}\text { Multiple } \\
\text { Knee w/wo } \\
\text { locking - and } \\
\text { Ankle }\end{array}$ \\
\hline
\end{tabular}




\section{Chapter 7}

\section{Compliant Actuation on Lower Limb Exoskeletons}

Current over-ground gait exoskeletons are characterized by providing power to the main joints by means of stiff actuators. A trade-off between size and functionality results in the actuation of the main joints in the sagittal plane. By actively controlling Hip, Knee and Ankle in some devices, successfully provide walking assistance in subjects with locomotion impairments. However, these mechanisms lack primarily from compliant properties associated with safer human-machine interactions, and natural like actuation. Robots of the present, and future are not supposed to be solid, isolated and rigid anymore but rather adaptive, cooperative and compliant entities in our daily life. Compliant joints appeal as a must in robotic exoskeletons, because of their ability to minimize large forces due to shocks, to safely interact with the user, and their ability to store and release energy in passive elastic elements. Also, by adjusting the natural dynamics of a mechanical system, it will have a natural motion close to the desired motion that could be translated into reduced energy consumption.

This chapter addresses the selection and implementation of proper compliant actuation systems into the joints of ATLAS exoskeleton. The evaluation of the behavior of the compliant exoskeleton; as well as the analysis, and discussion of the results obtained during walking tests with subject specific anatomic models, will be tackled on this chapter.

\section{1. -ATLAS $1^{\text {st }}$ Prototype- a Stiff Actuated System}

The ATLAS exoskeleton was conceived for children's gait assistance, and it can be considered as an active THKAFO (Trunk-Hip-Knee-Ankle-Foot Orthosis). ATLAS is intended to support users up to $40-\mathrm{kg}$, and heights from $130 \mathrm{~cm}$ to $165 \mathrm{~cm}$. The exoskeleton device allows the wearer to walk at low speed over a flat regular surface. It has 6 actuated degrees of freedom (DOF), having 3 DOF per leg; hip, knee and ankle providing movement in the sagittal plane. Figure 
7-1 shows an image of the ATLAS prototype containing 6 stiff actuators to provide the required power during locomotion to the 6 sagittal joints. The device presents a lightweight mechanical structure of approximately $10 \mathrm{Kg}$ including actuators. The user body is attached to the structure through comfortable belts, at the shank, tight, and torso. The flexion and extension motion of the hip, knee and ankle joints are driven by electrical brushless Maxon motors in combination with harmonic drive units. The setup can provide repeatable peak torques up to $76 \mathrm{Nm}$, and average torques of $32 \mathrm{Nm}$ at more than $20 \mathrm{rpm}$.

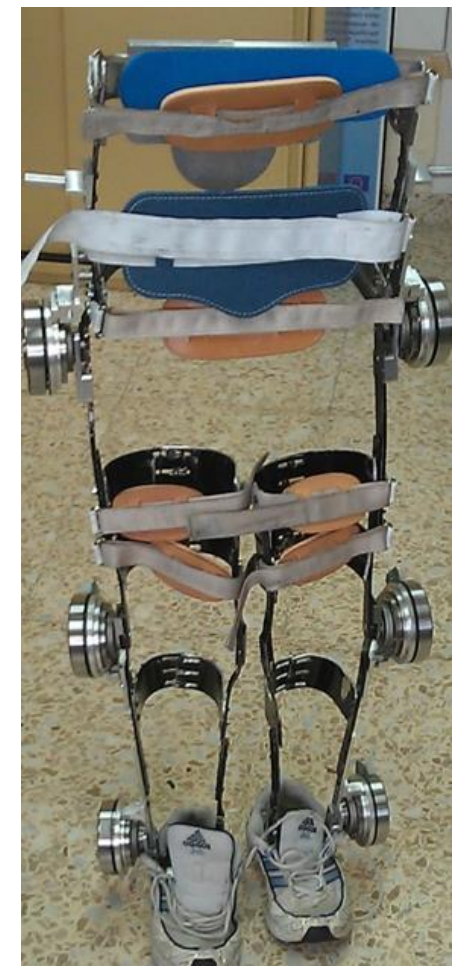

Figure 7-1. Exoskeleton Prototype with Stiff Actuation

The implemented motor-gearbox set provides large power-to-weight ratio while keeping flat enough for not to bother the user motion. However, the large reduction ratio results in very stiff joints not desired when interacting with humans and in presence of cyclic impacts, like those experienced during heel-strike, and changes of directions of the links.

The systems should have the capability of torque-controlling joint motion, and the ability of adaptability, and providing a safe environment for human-machine interaction are main goals when developing a fully functional active exoskeletons for the full-body paralyzed, such as 
quadriplegics, and subjects with Muscular Dystrophy; especially when children are the intended users as in the case of ATLAS exoskeleton. The target users of the device bring the added difficulty of requiring dynamic-stability control, as many of these subject do not have thoracic control, nor can be assisted by means of crutches or common walkers like in many of the commercial devices available for adults.

During the development of the ATLAS project, the balance assistance, needed due to lack of strength or mobility in the arms, were tackled by developing an especial walker capable of attaching itself to the exoskeleton, avoiding the need of using crutches or conventional walkers. The device was designed to provide stability in axial and frontal planes, and a detailed description of this stabilizing walker can be obtained from the author's patented document [8] ES2459866B1. M. Cestari et al (2014). "ANDADOR CON MECANISMO DE ASISTENCIA EN OPERACIONES DE LEVANTADO Y SENTADO DE UN USUARIO”.

The walker was equipped with a mechanism allowing the user to sit and stand while dressed with the exoskeleton. Another mechanism embedded in the walker, provides the freedom of vertical displacement with respect of the walker, maintaining the axial and frontal planes constrained. The vertical displacement, allowes the natural motion of the Center of Mass of the subject, during the locomotion cycle. Figure 7-2, presents a CAD representation of a simplified walker frame, comprising the mechanism that allows the required motion up and down in the sagittal plane.

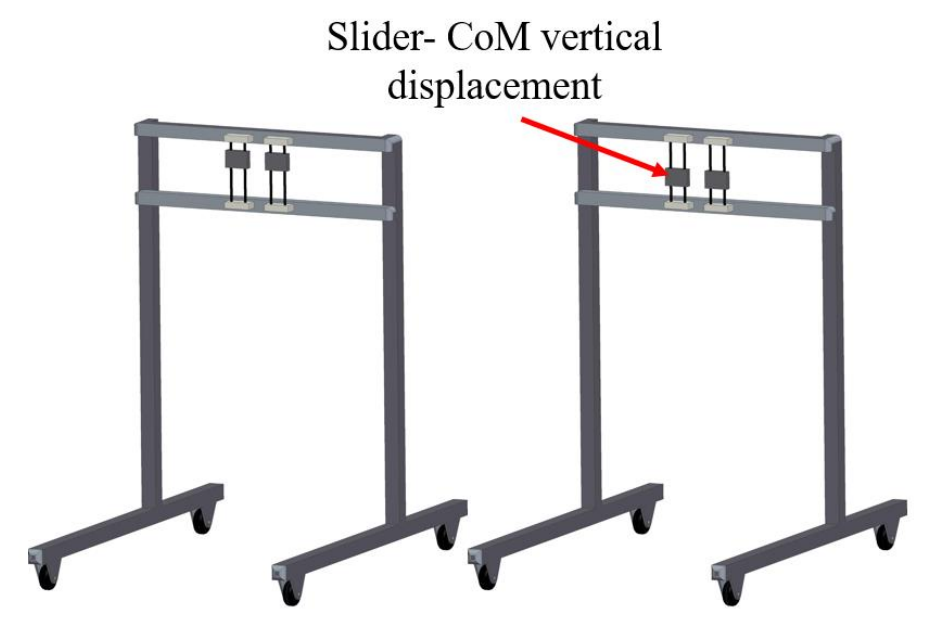

Figure 7-2. Walker Frame for Balance Control and Center of Mass Vertical Motion. 
The simplified realization of the walker device, was implemented to perform evaluations of the exoskeleton: when walking in straight line in the laboratory. The slider connected to the main frame, is attached to the back support of the exoskeleton, constraining motion in the lateral and frontal planes as previously stated. A displacement of about $5 \mathrm{~cm}$ is allowed in the vertical direction, associated with the CoM displacement during gait.

This first prototype of the ATLAS exoskeleton presented stiff joints, this original design intended to be light weight, and avoid bulky structures primarily in the lateral directions. However, the cyclic motion and constant impacts due to the ground contact, that characterize the gait, could produce loads on the stiff system that affect the life of the mechanical components.

The users of these robotics exoskeletons, often suffer from spasmodic movements and joint rigidity. For this reason, the sole position tracking results unadvisable, as it could produce damages to the subject joints. Impedance control needs to be implemented into robotic exoskeletons, with constant measuring of the torque at the joints, combined with its kinematics the impedance can be tune by means of control and constant drain of energy by the actuation systems. Muscles and tendons, are inherently compliant and are capable of modulating the impedance at the joints with little energy cost, compared to traditional impedance control by software.

By implementing in the ATLAS exoskeleton actuators with inherent compliant, several improvements to the system could be experienced. The shock absorption capability, associated with compliant systems could result in better life expectancy of the mechanical components. Also, adaptability to the subject pathology as well as adaptability to the environment can be achieved, and translated to safer user-machine interaction. These reasons, combined with the possibility of implementing special control strategies to exploit the gait dynamics and actuators compliance, in order to improve adaptability, a more natural walk, and energy consumption have encouraged the development of an inherent compliant exoskeleton.

\subsection{Compliant Model of the Leg}

In order to provide the desired compliant properties to the ATLAS exoskeleton; based on the biomimetic analysis conducted in Chapter 3, and focusing on the sagittal joints of the robotic device, a model of the leg combining hip-knee-ankle is presented in Figure 7-3. The figure shows 
the compliant behavior of the joints during a dynamic locomotion cycle based on the data from CGA [53]. The hip joint is characterized by cyclic generation of positive and negative power. The knee and ankle joints present a wider range of actuation, with several regions of energy absorption that can be reutilized for the generation of power in different areas of the gait.

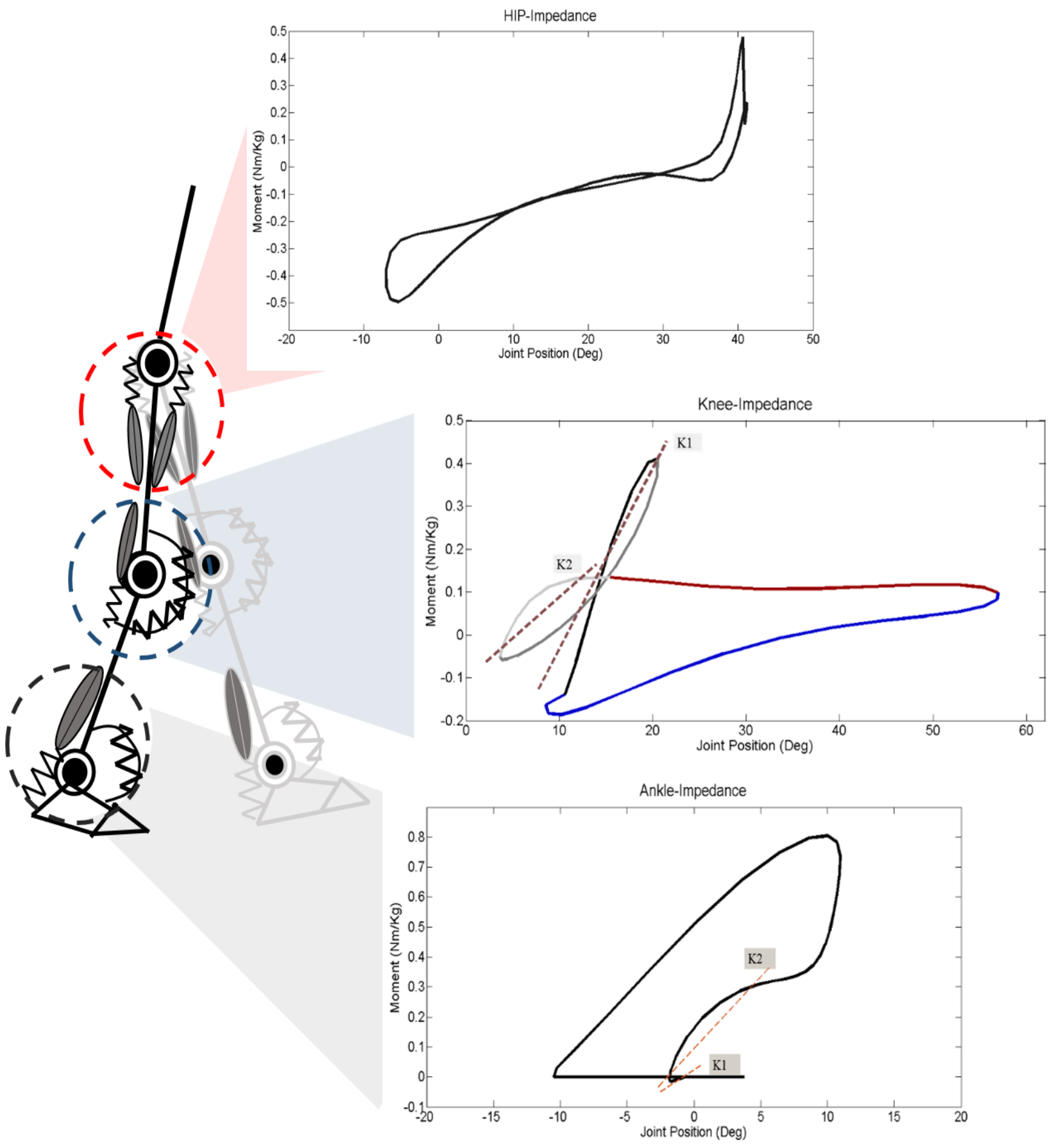

Figure 7-3. Leg Model- Levels of Stiffness 
In the previous chapters, two compliant actuation systems were presented and evaluated. Both systems have similar characteristics in term of its main components, components arrangement, torque tracking capabilities, and size. However, ARES-XL (System 2 - S2) outperforms the ARES (System 1 - S1) system in range of stiffness and allowable deflection. These properties shown in Figure 7-4, result of high interest for selecting an adequate actuation system for the different joints of a compliant robotic exoskeleton.
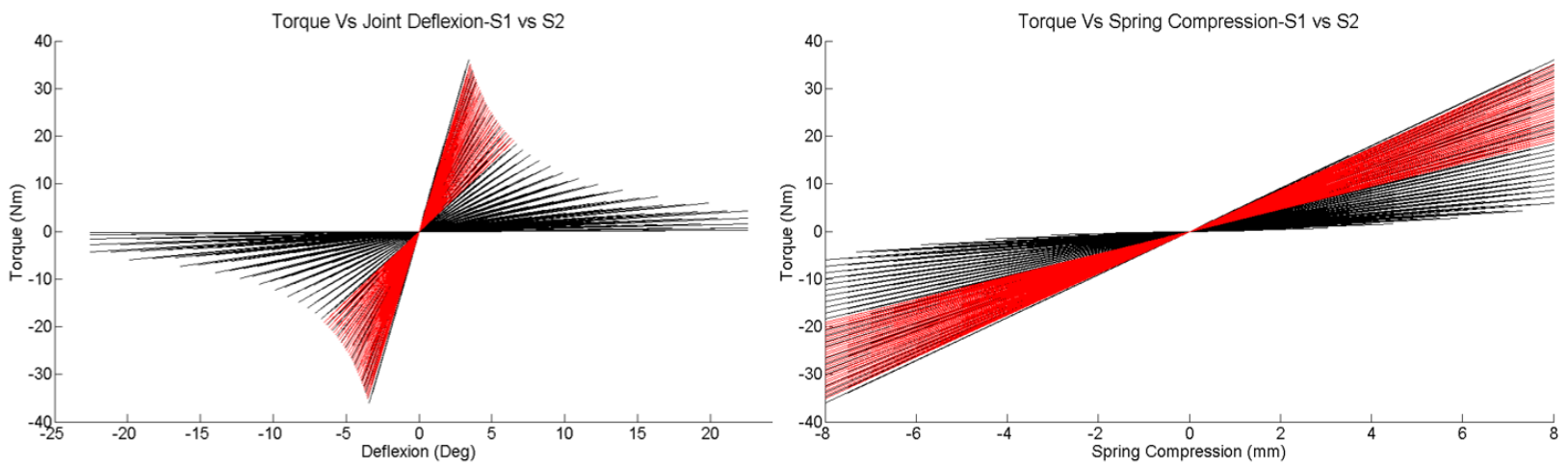

Figure 7-4. ARES vs ARES-XL Stiffness Ranges for System Selection

\subsubsection{Hip Actuation}

Based on the requirements at the hip joint, a simple bidirectional actuator with intrinsic compliance should be sufficient. ARES actuation presents a simpler mechanism compared to ARES-XL, making the $1^{\text {st }}$ system the preferred option to implement at this highly actuated joint. However, from Chapter 3 where a cyclic behavior at the hip joint is observed, no significant stiffness modulation occurs during gait. For this reason, a simplification of ARES actuator is introduced in Figure 7-5. Replacing the slider element by a Fixed Element attached to a manually adjustable distance from the joint axis, and reducing the size of the slotted bar $-\mathrm{S} 1 \mathrm{~B} 1$ an actuator with intrinsic compliance is obtained. This simplification of the ARES mechanism, basically conform a rotary actuator with Series Elasticity. The main working principle of ARES and its torque measuring capabilities are maintained. 


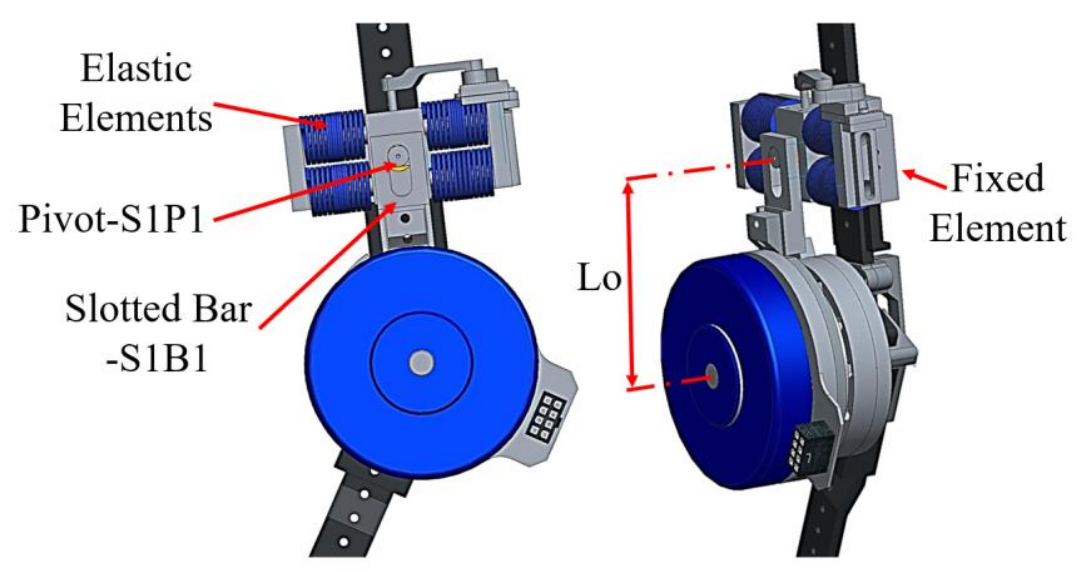

Figure 7-5. ARES-Simplification, a SEA Rotary Actuator for the Hip.

The associated relationship to calculate the torque at the joint, is the same used for ARES keeping Lo as a constant value. The implemented springs provide an equivalent elastic constant of $88 \mathrm{Nm} / \mathrm{mm}$ and the constant distance Lo for this particular realization will be $70.5 \mathrm{~mm}$. Therefore the hip joint torque is given by:

$$
\tau_{H I P}=\frac{2 \cdot L_{0} \cdot \Delta X \cdot K}{(\cos (\gamma))^{2}}
$$

Where,

$\Delta \boldsymbol{X}$, corresponds to the elastic element's compression, measured by the linear encoder.

$\boldsymbol{\gamma}$, the joint deflection due to the elastic elements compression.

$\boldsymbol{K}$, is the equivalent rigidity of the elastic elements in the slider element $(\mathrm{K} \sim 88 \mathrm{Nm} / \mathrm{mm})$.

$\boldsymbol{L}_{\mathbf{0}}$, is the distance between the joint axis and the pivot $-\mathrm{S} 1 \mathrm{P} 1(70.5 \mathrm{~mm})$.

\subsubsection{Knee Actuation}

The knee is a versatile joint, with higher torque generation in the extension direction than in the flexion, during the gait cycle. This joint acts as a compliant passive joint during several segments of the locomotion cycle. Based on Chapter 3 and Figure 7-3, the presence of different 
stiffness levels is evident at the knee motion. The implementation of a system capable of adjusting its stiffness during the gait cycle is desired at the knee.

ARES-XL with locking mechanism, is chosen due to the large deflection, minimum stiffness and possibility of implementing a locking mechanism to store energy and/or lock deflection to be later released. Large compliant capabilities can be exploited during loading response of the knee. Later in the cycle the flexion of the knee at the beginning of the swing can occur while maintaining the deflection during pre-swing, by engaging the locking mechanism. Finally allowing a partially natural swing by achieving zero stiffness in the proper moment of the gait (end swing), taking advantage of the leg inertia and kinematics.

An extra elastic element can be implemented in the compliant mechanism to increase the resistance in the extension direction. Thus the Fs used to calculate the exerted torque in the ARESXL will correspond to a piece wise force, were the regular spring constant will be $16.8 \mathrm{~N} / \mathrm{mm}$. The extra springs will only act after certain compression is achieved given the following relationship for the extra spring constant.

$$
\left\{\begin{array}{c}
K_{p w}=0, \quad \Delta X<X_{o} \\
K_{p w}=22 \frac{N}{m m}, \quad \Delta X \geq X_{o}
\end{array}\right.
$$

The resulting relationship for the torque calculation is given by (See Figure 6-5),

$$
\tau_{\text {Knee }}=\frac{\left(2 \cdot \Delta X \cdot K+\left(\Delta X+X_{o}\right) \cdot K_{p w}\right)}{\cos \alpha \cdot \cos (\beta)} \cdot \frac{L_{2} \cdot L_{1}}{L_{4}}
$$

Where,

$\Delta \boldsymbol{X}$, correspond to the springs' compression, measured by the linear encoder.

$\boldsymbol{\alpha}$, and $\boldsymbol{\beta}$ the bar $-\mathrm{B} 2$ and joint deflection due to the springs compression.

$\boldsymbol{K}$ and $\boldsymbol{K}_{\boldsymbol{p} \boldsymbol{w}}$, are the equivalent rigidities of the elastic elements in the slider device.

$\boldsymbol{L}_{\mathbf{2}}$, is the distance that can be adjusted by M2, between the joint axis and the slider device.

$\boldsymbol{L}_{\mathbf{4}}$, effective arm length between the pivot in the slotted bar-B2 and the coupling point.

$\boldsymbol{L}_{\mathbf{1}}$, fixed distance, from the joint axis to the pivot $-\mathrm{P} 1$ in the coupling point. 


\subsubsection{Ankle Actuation}

Typically implemented as a passive joint in several exoskeletons, the ankle characterized by adapting to the reaction of the ground and the user weight. The dorsiflexion motion during the support phase, corresponds to the reaction of the joint behaving as a compliant passive system. In a dynamic gait, during the toe-off the push up occurs. This motion, produces a sudden change of direction in the joint with a power profile similar to an impulsive force applied to the foot. The energy required during this phase can be provided in part by the energy stored at the support phase, and by the main actuation system combined. The main difficulty is associated with the acceleration required by this impulsive power, typically not achievable with electric motor-gearbox combinations. Based on the evaluation of ARES-XL in chapter 6, by allowing a large deflection during the support phase, followed by a sudden increase of stiffness in the compliant mechanism just before the toe-off; a high impulse can be delivered to the joint, leaving the main actuator with less power to deliver. ARES-XL without need of locking mechanism, will be implemented at this joint because of its larger deflection- and energy storing capability. Similar to the plain ARES-XL with identical springs of $16.8 \mathrm{~N} / \mathrm{mm}$ constant, the torque relationship is given by.

$$
\tau_{\text {Ankle }}=\frac{2 \cdot \Delta X \cdot K}{\cos \alpha \cdot \cos (\beta)} \cdot \frac{L_{2} \cdot L_{1}}{L_{4}}
$$

Eq. 7-4

\subsection{ATLAS Exoskeleton- Compliant Joints}

The ATLAS exoskeleton was equipped with compliant joints in its left limb, while maintaining the traditional stiff actuators in the right side, to allow comparison of compliant/non compliant behavior. Figure 7-6, presents the compliant joints implemented in the exoskeleton prototype and a CAD view highlighting the systems in use. At the hip the simplified version of ARES will provide compliant and torque measuring properties, maintaining a reduced size. Two versions of the ARES-XL are implemented to the lower joints, one at the knee including the addon locking mechanism and the plain version of the ARES-XL at the ankle. The exoskeleton will be evaluated by programing a locomotion cycle under different conditions, to analyze the data collected from the different feedback sensors. 


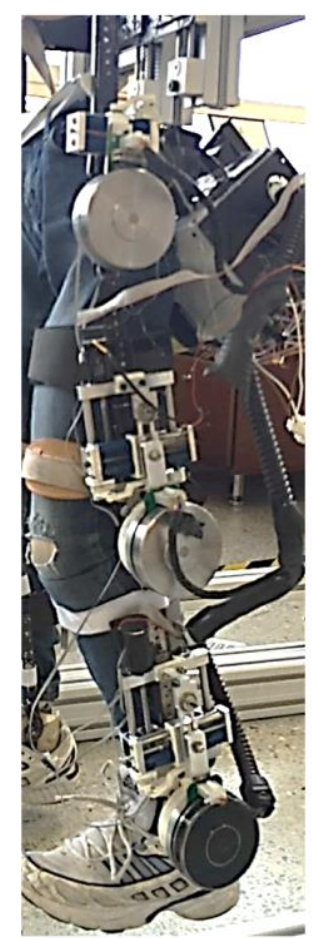

(a)

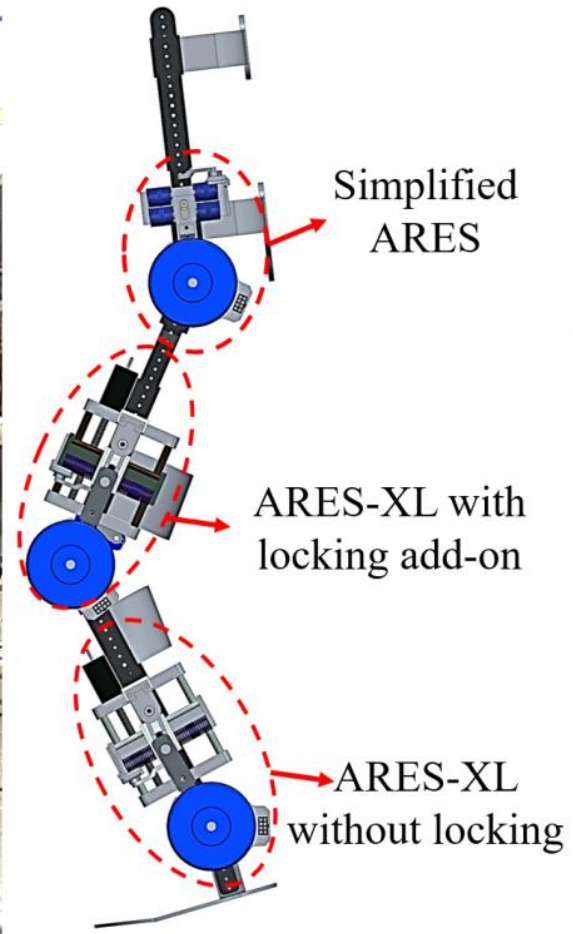

(b)

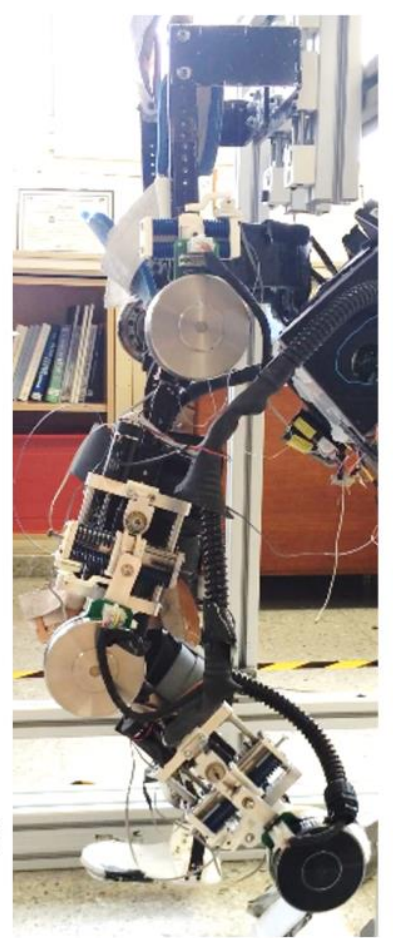

(c)

Figure 7-6. ATLAS Exoskeleton- Prototype with Compliant Joints.

\subsubsection{Experimental Setup}

The assessment of the compliant exoskeleton was made with the assistance of the simplified Walker frame for balance. The back of the exoskeleton was connected to the slider in the walker, allowing the required mobility for the CoM displacement, see

Figure 7-7. In order to emulate the subject in the exoskeleton, a test dummy having limb mass, length, and inertias of a 10-yo child was used. The target subjects for these robotic devices, are sensitive subjects, that can-not be exposed to the preliminary evaluations perform in these experiments. However, the use of a test dummy, with matching properties to a child of 10 years old, results in a suitable option to conduct the experiment. The test dummy presents a socket joint at the hip, hinges at knee and ankle, and flexible feet. Similar to the target users, the dummy is not capable of providing balance to the system, thus the combination of exoskeleton, walker, and control system is required to achieve a forward human-like gait. 


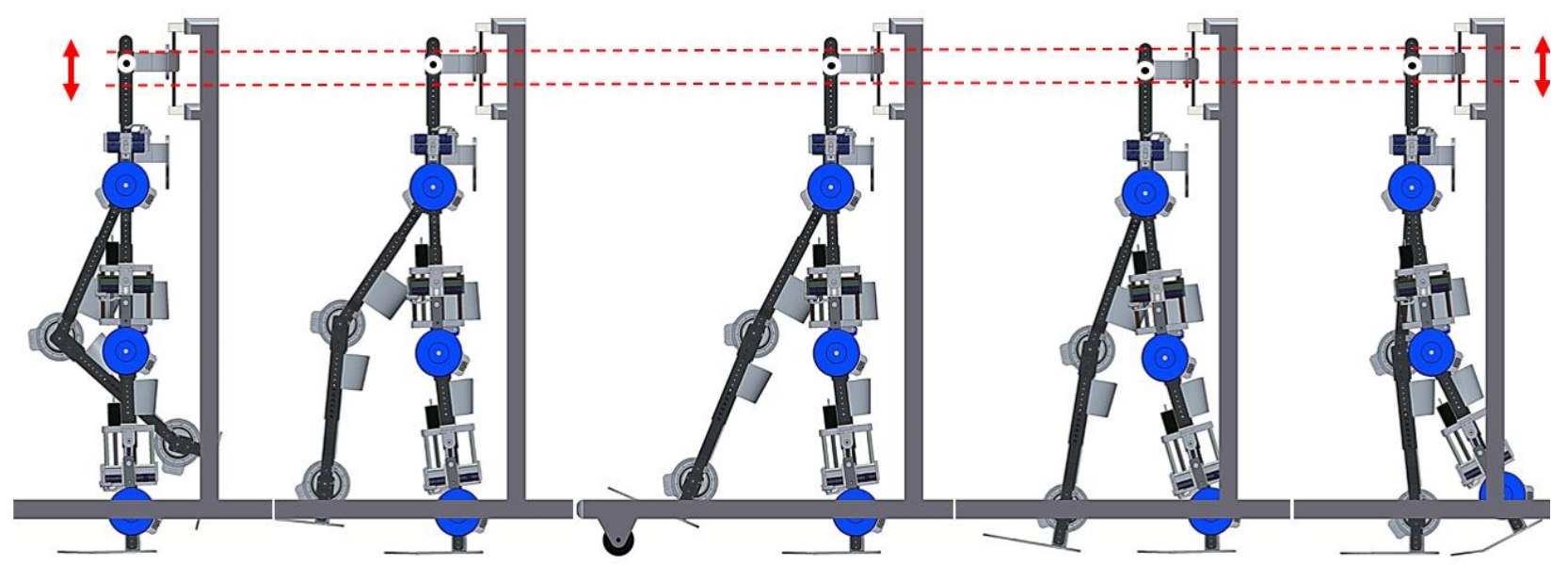

Figure 7-7. Exoskeleton and Walker Frame Coupled. CoM Vertical Displacement During Gait.

\subsubsection{Hardware Architecture and Basic Control}

The exoskeleton is equipped with a My-RIO, an on-board controller from National Instrument. This hardware, presents a reduced size and various inputs/outputs that allows the control and constant communication with the exoskeleton controllers. In Figure 7-8, a simplified diagram of the implemented control is shown. The MyRIO can communicate to the main PC via wireless communication, in order to transfer the logged data from the exoskeleton components. Compliant joints are implemented in the left link, while stiff actuators provide the power to the right limb. ESCON 50/5 controllers are used to command the motion to the main motors $-\mathrm{M} 1$, while EPOS 24/5 are used to control the position of the slider elements in the compliant mechanism by means of $-\mathrm{M} 2$. Each exoskeleton joint is equipped with magnetic encoders AS5045, via SPI communication. The magnetic sensors are used as feedback of the real joint positions.

From the exoskeleton hardware and instrumentation, position, set points, stiffness level, spring's compression, and current consumption are continuously collected and logged during experiments for later analysis. 


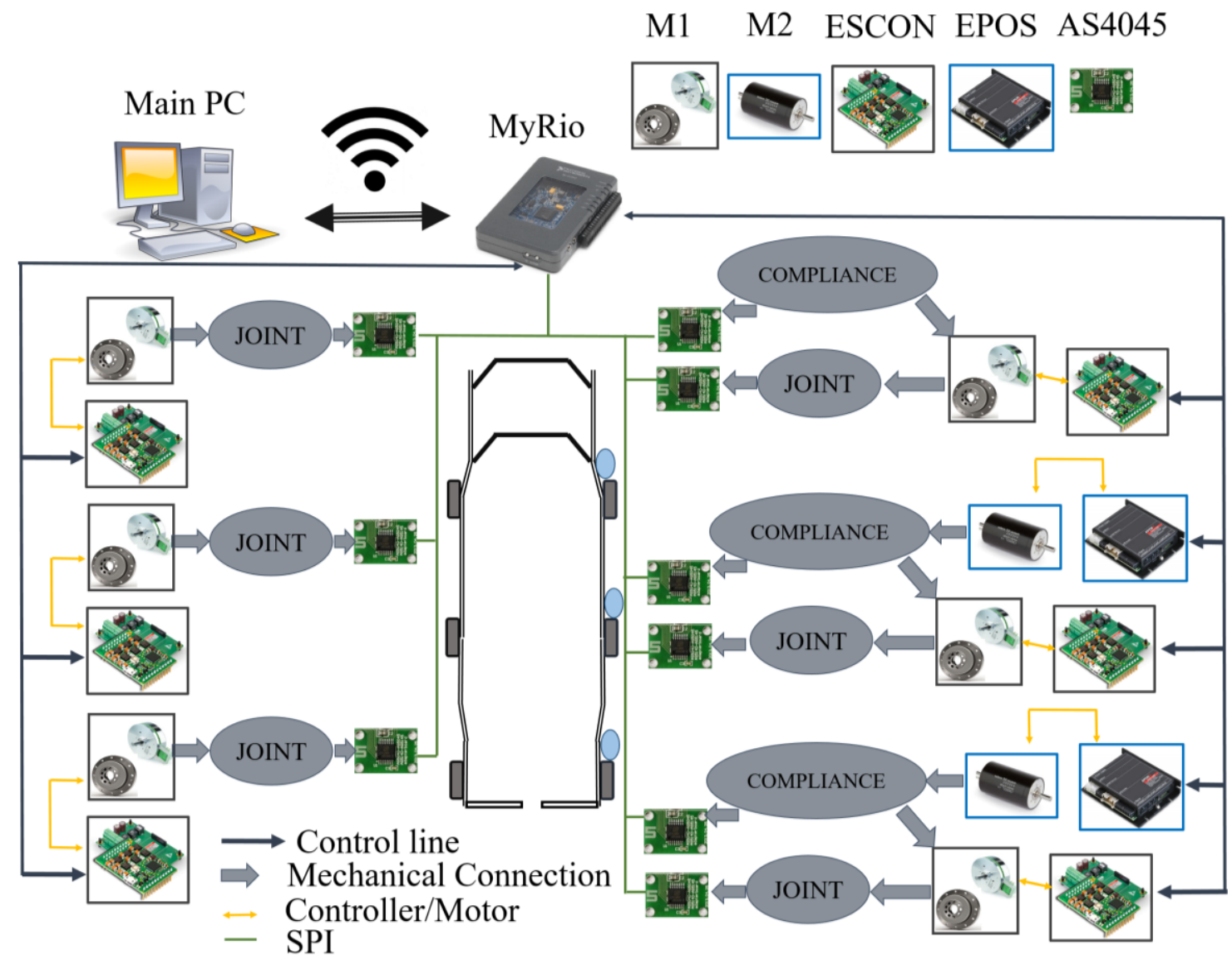

Figure 7-8. Simplified-Control Diagram of the ATLAS Compliant Exoskeleton.

The implemented control is based on an articular position control-scheme, where predefined trajectories based on healthy subjects' data are commanded to the exoskeleton's joint. The length of the steps, as well as the speed of the gait, or cadence can be adjusted. The inverse kinematics of the exoskeleton is used to validate the desire positions and the valid articular angle sent as reference to the particular joint of the exoskeleton. Figure 7-9 presents the basic control scheme implemented at the exoskeleton's joints. The joint position is a function of the system deflection, ground reactions, and main motor position. 


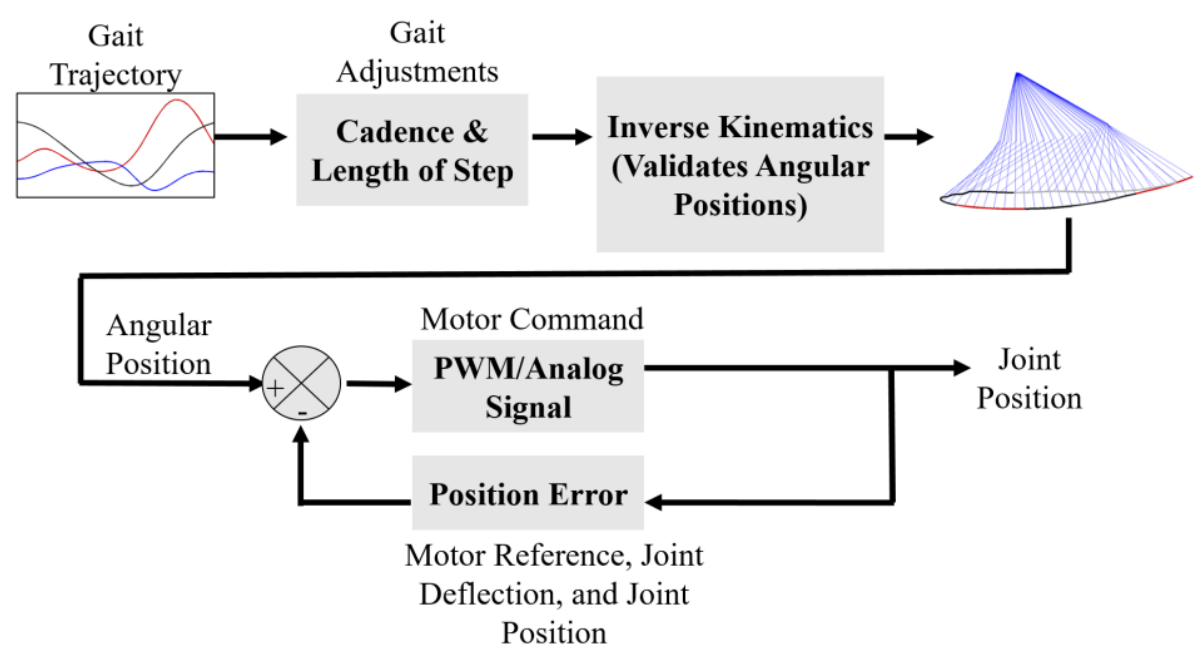

Figure 7-9. Basic Control Diagram

\subsubsection{Trajectory tracking}

A predefined trajectory based on CGA data was commanded to the exoskeleton and shown in Figure 7-10, in order to evaluate the behavior of the compliant joints during the gait cycle. The robotic exoskeleton is actuated only in the sagittal plane, and as a consequence some considerations need to be taken into account during the locomotion cycle.

The walker constrains the lateral and frontal motion, and as a consequence of this the abduction required for weight shifting during gait can-not be provided. This motion wouldn't be possible when experimenting with a test dummy or the intended subjects, that are not capable of providing this motion by themselves. In order to tackle this issue, the knee flexion during swing needs to provide mostly the ground clearance.

The commanded gait trajectory, to the exoskeleton is at low speed, thus as shown in Figure $7-10$, in order to assist the ground clearance and the sagittal stability, the leading leg will remain still while the hip in the leg in the air achieves the maximum forward position, and the end-swing begins. The commanded trajectory, aims to mimic a natural-like gait, with an extended support phase to assist the ground clearance during the slow gait. 


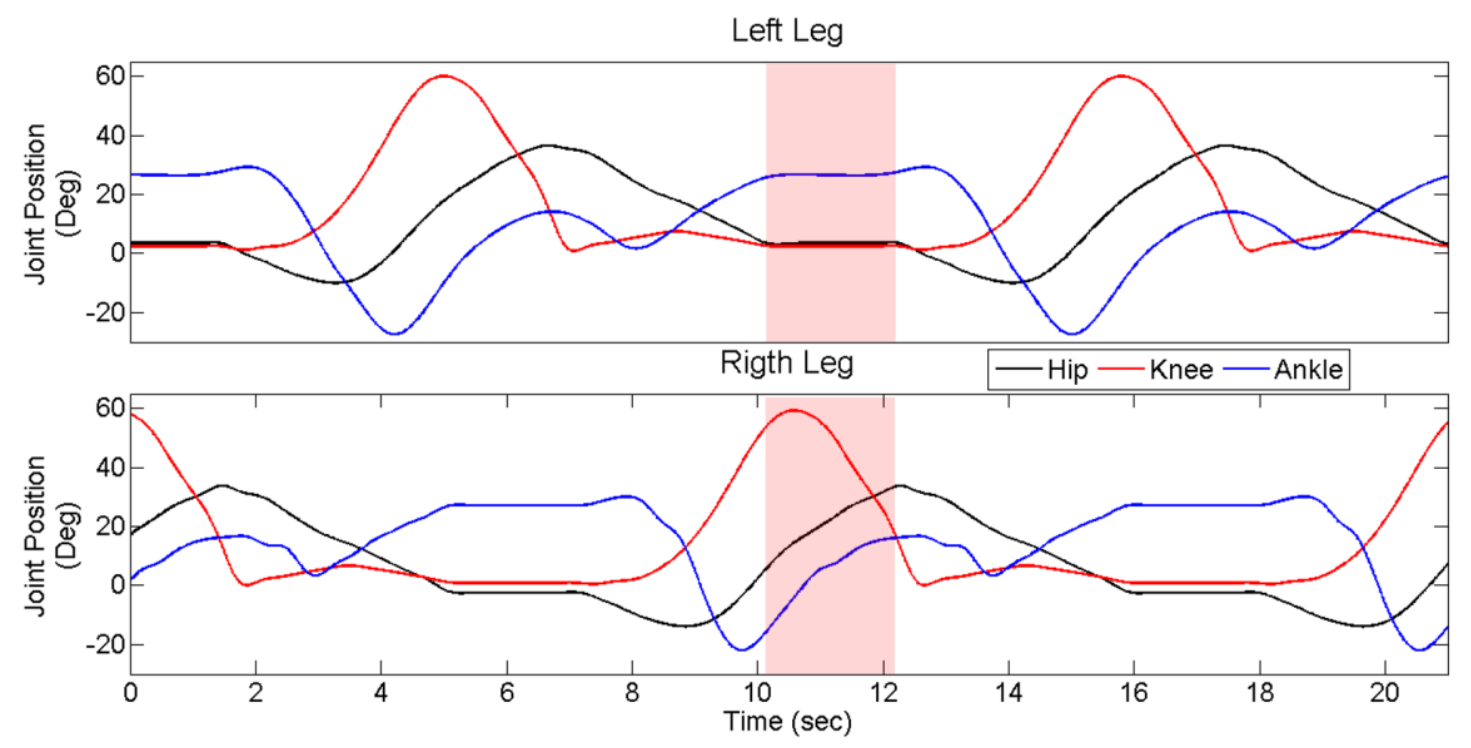

Figure 7-10. Commanded Joints Trajectories. Highlighted Area Where Leading Leg Waits for the Leg Swing of the Other Leg.

\subsubsection{Torque Tracking During Locomotion}

Taking advantage of the embedded force sensor in the implemented actuators, the torques during the commanded walking cycles were calculated and the results analyzed for discussion. The relationships Eq. 7-1, Eq. 7-3, and Eq. 7-4 are used to calculate the exerted torques at the joints.

The lower limb of the test dummy was used as load in the exoskeleton. With an equivalent weight near to $20 \mathrm{Kg}$ and articulated hip, knee, and ankle; the compliant exoskeleton was evaluated under different stiffness levels: medium, high, and completed stiff. By blocking the elastic elements in the compliant mechanism, a complete stiff configuration can be achieved for comparison purposes.

\subsubsection{System with Load-No Ground Contact}

With the lower limb of the test dummy placed, and strapped inside the exoskeleton, the stabilizing walker and the exoskeleton already coupled were placed over a couple of steps in order to maintain the feet of the exoskeleton away from the ground. 
Three stiffness levels were tested. Medium, and High Stiffness at both ARES-XL actuators at knee and ankle, and compliant hip. A Stiff configuration at the 3 joints is achieved, by mechanically blocking the elastic elements in their compliant mechanism to complete the third stiffness level (Stiff).

The main objective of these experiments, is to evaluate the behavior of the torques exerted at the joints, during the non-existing loading phase. As well as the effects in the trajectories followed by the joints under different stiffness levels.

The results from the torque, and joint position tracking for the hip are presented in Figure 7-11. The hip can only present stiff behavior by blocking its compliance, and high stiffness. Both plots remain fairly constant along the gait and under the different configurations of stiffness. Although when the joint is fully stiff, the torque tracking capabilities can-not be used, the stiffness variation in the other joints didn't affect the torque exerted by the hip.

Torques about $20 \mathrm{Nm}$ are generated for flexion and extension at the hip. The highlighted region points a section of the trajectory where the leading leg remains stills waiting for the other leg to achieve ground clearance. No significant angular variation, occurs in this region, and the

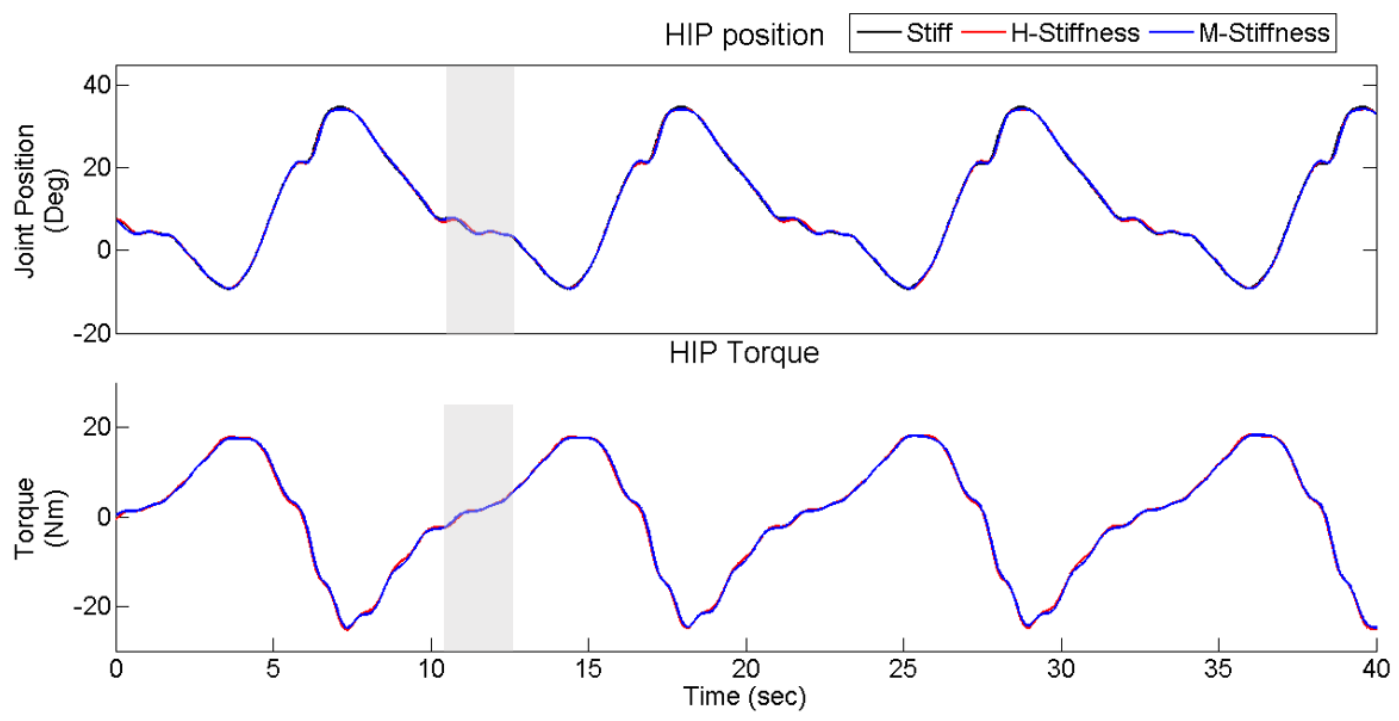

Figure 7-11. Torque Tracking Under Different Stiffness for Knee and Ankle Joint- Hip in the Air With Load. 
torque exerted is near zero, compatible with the leg being in almost vertical position and no torque required to maintain the position.

The knee joint, actuated by ARES-XL was evaluated at 2 levels of stiffness besides the complete stiff configuration. Figure 7-12 present the results obtained from the joint, with a gradient highlighted section, indicating the sub phases of the knee. Loading and pre-swing don't show torque variations or increments, which is logical when no ground contact is part of the experiment. These phases of the knee, are consequence of the coupling between ground and the contact of the leg with it, by means of the foot. The Low torque in the extension direction, corresponds to the required torque to extend the dummy's mass of shank, and foot. This torque is significantly lower than the required at the beginning of the swing, where the knee flexion produces a higher projection of the lower leg mass.
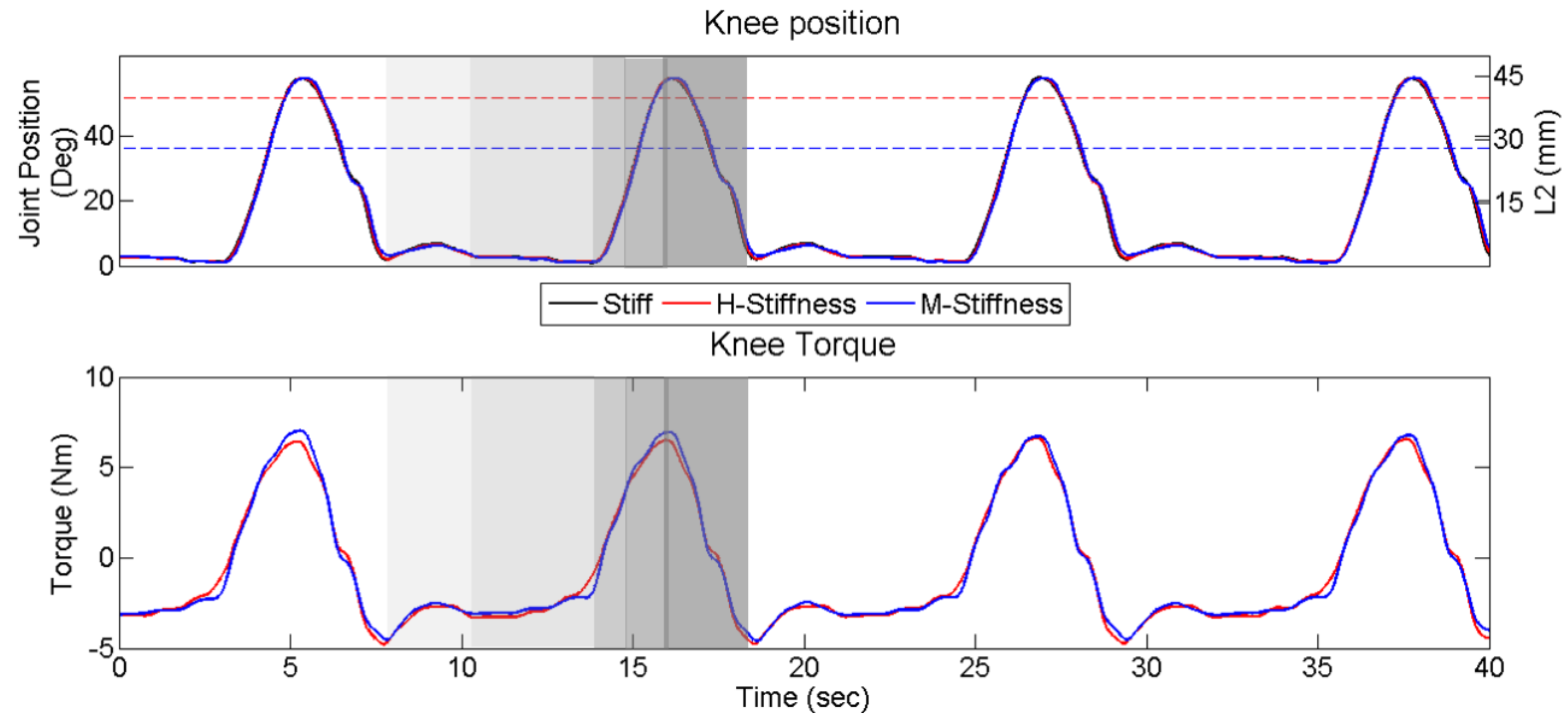

Figure 7-12. Torque Tracking for Different Stiffness- Knee in the Air With Load.

The torque at the ankle was considerably low when compared to the other joints, this is understandable because only the foot's mass is affecting this joint in the experiments in the air. Figure 7-13 highlights two relevant sections of the ankle during gait. The first region, highlighted in gray shade corresponds to the loading of the foot, when the dorsiflexion allows adaptation of the body weight when in contact with the ground. In this experiment, because of the lack of ground 

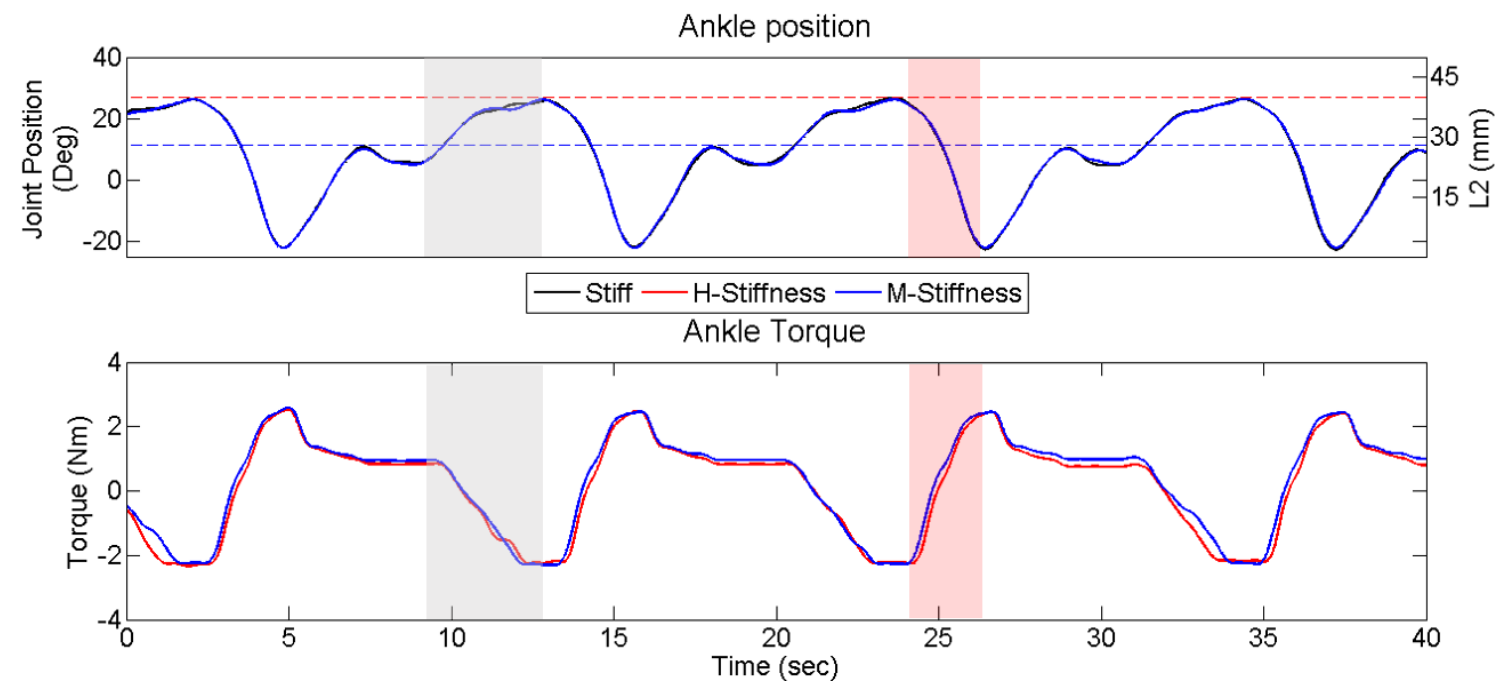

Figure 7-13. Torque Tracking for Different Stiffness- Knee in the Air With Load.

reaction, there is no torque increase at the torque exerted at the joint until the intrinsic stiffness of the foot opposes to the commanded dorsiflexion. The reddish section, indicates the push-off section, where during a dynamic gait cycle, a fast flexion occurs in the ankle causing a torque increment just before the foot leaves the ground. The flexion at the ankle, does not generate a torque increment in the experiment, because the lack of ground contact reacting to the fast movement.

\subsubsection{System with load- Ground contact.}

The steps from the previous experiments were removed, allowing the exoskeleton feet to be in contact with the ground. Constraining the movements of the exoskeleton's back, allowing only vertical displacement necessary for the CoM motion, the exoskeleton was commanded to walk in a straight line for about $5 \mathrm{~m}$.

During the locomotion cycle, the sections where the leading leg remains still assisting the ground clearance of the opposite leg, the inertia of the body produces an added load to the hip, similar to a shock caused by the deceleration of the hip to stop its motion. Figure 7-14 shows torque spikes during the deceleration of the leading leg, as a consequence of the body inertia. In the position plots, this torque spikes are evidenced as small deviations from the commanded position. When the hip is provided with compliance, the leg adapts to the added load. The stiff hip, does not present the same deviation that in the compliant case, which indicates that the impact is absorbed 

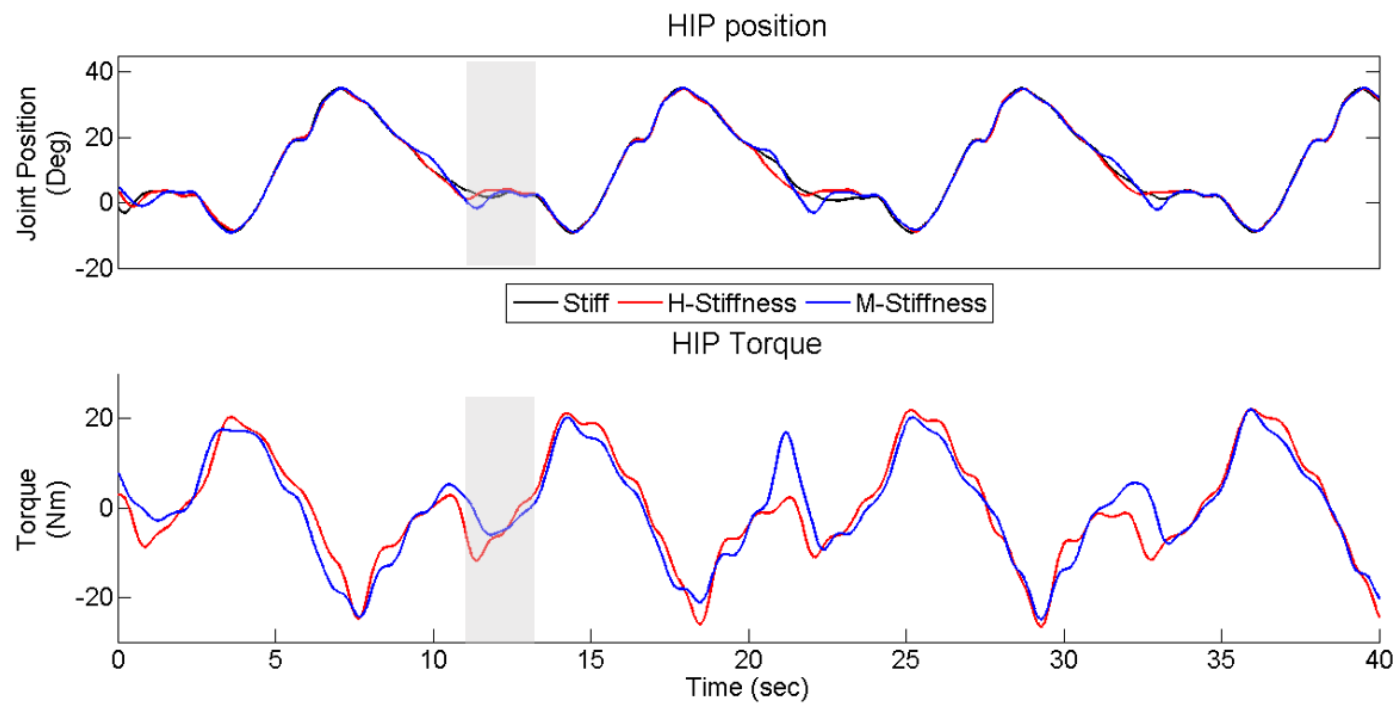

Figure 7-14. Torque Tracking for Different Stiffness at Knee and Ankle- Hip in the Ground With Load. by the mechanical structure. The implementation of the simplified ARES at the hip, allows the shock absorption of the inertial load, without significant deviation from the commanded trajectory. Stiff path tracking is not a requirement in exoskeleton applications. Adaptability to impacts and different loads is more desirable at this application, making the selected actuator an interesting and functional device to actuate the hip in the ATLAS robotic exoskeleton.

Figure 7-15 presents the results obtained from the knee, as expected during loading response, higher torques in the extension direction are exerted, compared to the flexion torque at the beginning of the swing. The torque immediately increases after the extension at the swing, as a consequence of the ground reaction and the joint acceptance of the body weight. All over the support phase and the pre-swing, the reaction between the ground and the exoskeleton produces significant torque at the knee.

Both stiffness levels present similar results, indicating both could be suitable for that torque range, and the desired deflection could be used to select the better choice for the application. Slight positions variation occur before the pre-swing when the leg remains still. These variations come along with small torque changes, indicating the knee adapts to the variation caused by the body and ground interaction.

During the beginning of the swing, the torque exerted change direction. Reaching its maximum for flexion, at the maximum flexion of the knee. 


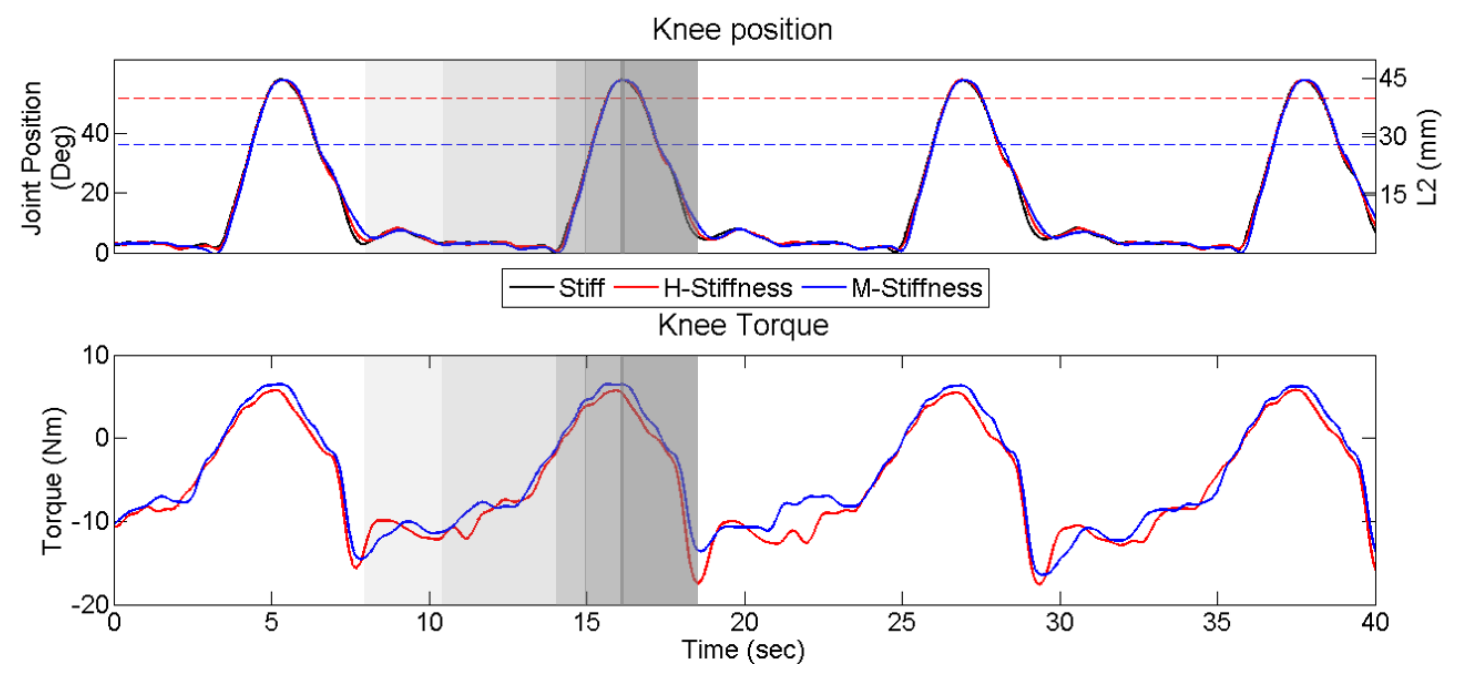

Figure 7-15. Torque Tracking for Different Stiffness- Knee in the Ground With Load.

The compliant ankle reaches a saturation torque during the support phase, however as can be noticed in the highlighted reddish area in Figure 7-16, where the push-off should occur, the torque decreases without a brief initial increment. It seems from these results that the foot leaves the ground before expected. The implemented gait trajectory intends to achieve a natural-alike gait, but the several constrains such as limited DOF and planes of motion limitations, can affect segments of the gait.

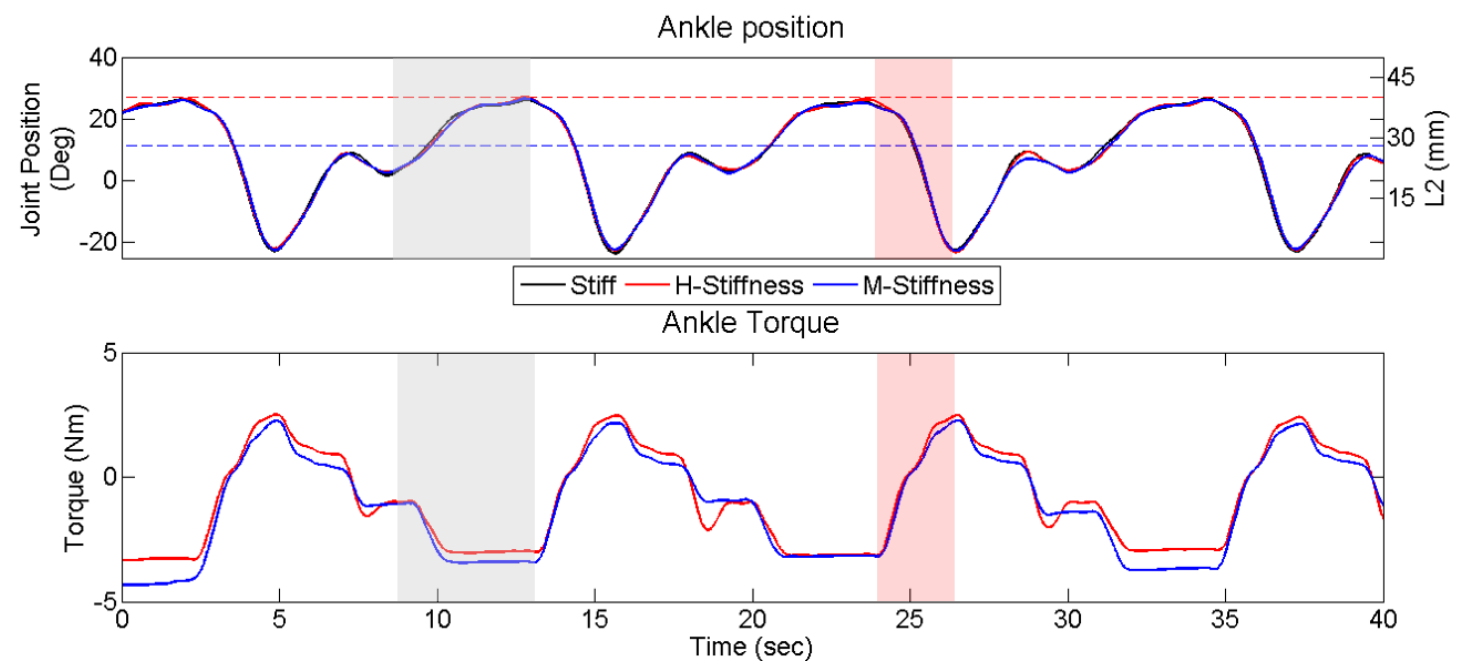

Figure 7-16. Torque Tracking for Different Stiffness- Ankle in the Ground With Load.. 


\subsubsection{Load Comparison}

Comparisons of the results obtained in the previous experiments between themselves and those obtained from the exoskeleton along, without the test dummy in it. The angular positions, and the torque calculated implementing a High level of Stiffness in both ARES-XL actuators is shown and discussed below.

In Figure 7-17, it can be evidenced that the test dummy requires most of the torque exerted by the actuators. The behavior of the joint when the exoskeleton is walking on the ground, presents small deviations from the trajectory as response to the inertia of the body. It is also appreciable bigger torque slopes during the ground experiments. The hip body takes longer in changing the torque direction, and later the body weight contributes to the torque generation when the body is on the motion. This behavior shows once again an adaptable behavior from the compliant hip joint, as a response of the body inertia and the commanded trajectory.

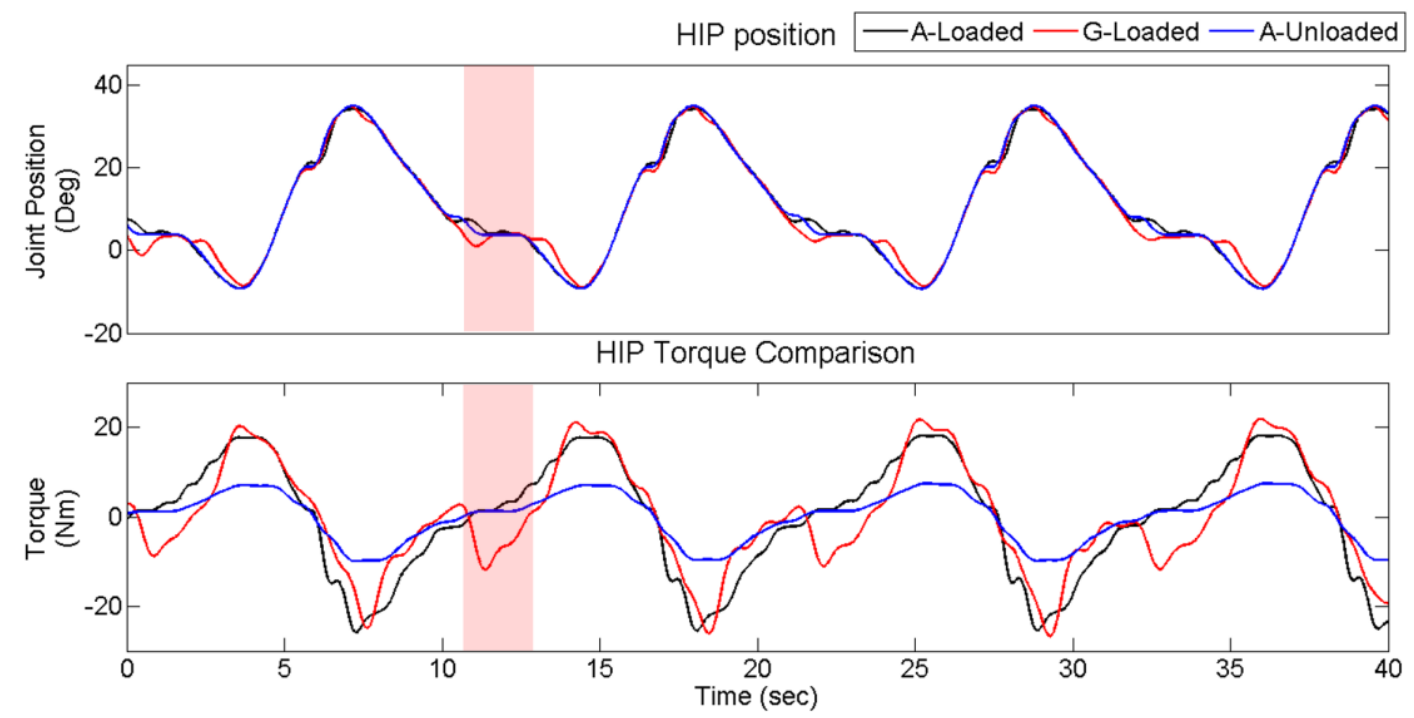

Figure 7-17. Torque Tracking at the Hip under Different Conditions of Load.

The knee torques exerted at the knee, are more affected by the reaction to the ground. The torque is directly related to the joint angular position only during the swing phase, but in the extension direction, the reaction to the ground produces a significant increment of the torque, when compared to the experiments on the air. This can be explained as a reaction of the joint to the acceptance of the body weight all over the support phase. Figure 7-18 presents the joint positions, torques and joint deflections during the 3 experimental conditions. Three sections are highlighted, 
corresponding to an extended loading response area, corresponding to the heel-strike up to before the swing. During the swing, the torque changes direction, as well as the joint deflection. However, this deflection previous the swing (pre-swing), it's being wasted. From the experiments in the previous chapter, as well from the response of the joint to different stiffness, this deflection could be stored by implementing the add-on locking functionality, or also by incrementing the stiffness at the joint previous the swing, thus less deflection gets wasted.

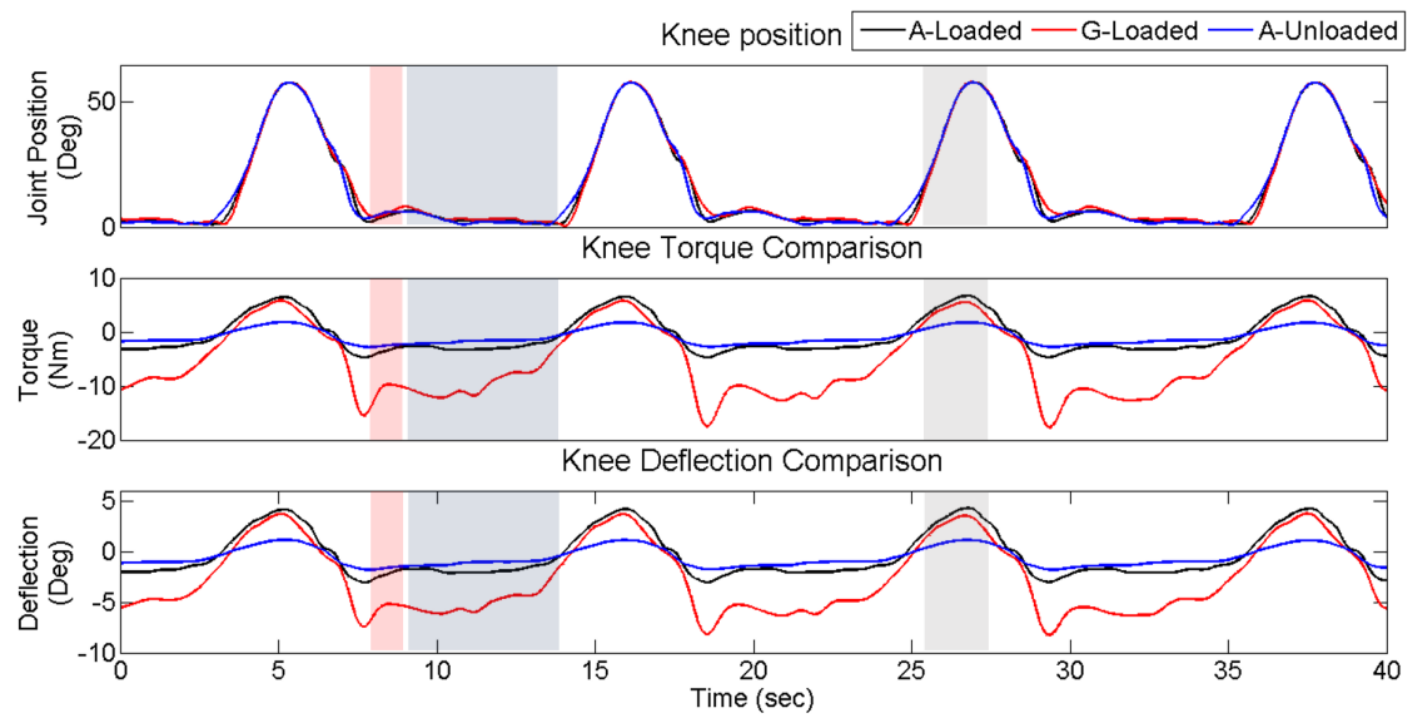

Figure 7-18. Torque Tracking at the Knee Under Different Conditions of Load.

The ankle joint torques and deflections profiles, when the test dummy in placed on the exoskeleton, present similar behaviors, as can be seen Figure 7-19. The torque required for flexion and dorsi-flexion are related to the foot resistance, more than to the ground reaction. During the heel-strike the reddish highlighted area shows the reaction of the joint. A small bump at the torque tracking plots indicates the foot adapts to the ground contact. Followed by the dorsiflexion of the foot due to the body weight, as indicates the presence of torque when ground contact is involved, prior to the experienced in the trial on air. This torque remains along the support phase, however at the push-off the torque instead of presenting a short increase before the foot leaves the ground, drops drastically to zero, continuing to changing direction. This indicates the foot is abandoning the ground before expected from the analysis conducted in chapter 3. This particular behavior, is endowed to the multiple constrains imposed to the gait in terms of Degrees of Freedom. 

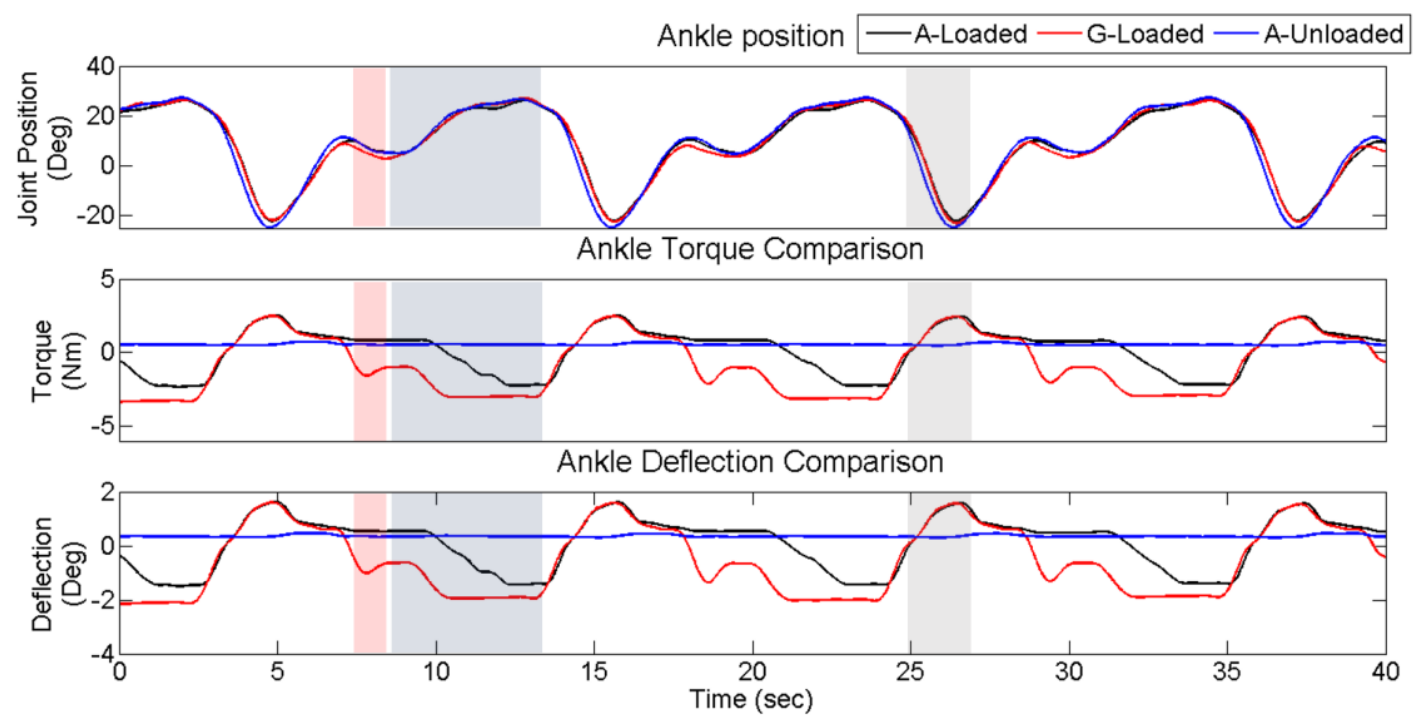

Figure 7-19. Torque Tracking at the Ankle Under Different Conditions of Load.

Several conditions were tested in this section, in Table 7-1 a summary of the most relevant results and observations are shown, comparing the behavior with different stiffness.

Table 7-1. Summary of the Torque Tracking experiment's Results.

\section{Condition Summary}

- The Hip, presented a sinusoidal behavior with no significant torque nor position peaks, other than in changes of direction.

- The Knee, characterized by no torque at loading response, as expected due to the lack of contact

Loaded in with the ground. The larger torque experienced was at the knee flexion, and no disturbances

the Air can be spotted in the results due to shocks or body reactions.

- The Ankle, little variation is visible at the ankle's torque. Only the foot inertia needs to be overcome in this evaluation. No push-Off occurs, as expected because there is not opposite reaction from the ground to the foot plantar-flexion

- The Hip, similar torques compared to the test in the air can be seen. Torque peaks can be noticed in the middle of the gait, when the leading leg waits for the other leg to reach the end of the swing. These peaks are evidence of shock reactions that need to be absorbed by the structure.

Loaded in - The Knee, shows evidence of loading response. Significant torque is experienced in the the Ground extension direction. The torque and position of the knee show adaptation to the ground, noticeable in small deviations from the reference in presence of compliance.

- The Ankle, presents slightly larger torque due to the ground reaction. However, no push-off can be observed, probably because the foot loose contact with the ground too soon. 


\subsubsection{Gait Control Strategy Based on State Machine.}

To evaluate the response of the exoskeleton's joints when online adjustments of the stiffness are made, a set of state machines were implemented at knee and ankle. The goal of these experiments is to assess the behavior of these joints during the main sub-phases of the gait, loading, pre-swing, and swing.

\subsubsection{Knee Implementation}

The versatile behavior of the knee joint has been discussed during Chapters 3, 5, and 6 . This joint is characterized by adapting to the ground during the support phase, as a consequence of the reaction of the ground and the loading of the body.

From the previous experiments with the exoskeleton, the torque tracking showed high torque during the loading response, and joint deflection at pre-swing. However this deflection is wasted during the beginning of the swing, when the directionality of the torque changes, associated with changes on the joint deflection. In order to evaluate alternatives, to either reutilize such deflection, or reduced to avoid the wasted energy, Figure 7-20 present 2 proposed state machine diagrams to implement at the knee.

The first control strategy named "Var-L1", presented in Figure 7-20 a) intends to exploit the deflection at the pre-swing, by means of the locking mechanism set to be engaged at around 10 degrees of deflection in the extension orientation. Once the knee is completely flexed at the swing, the lock can be disengaged by decreasing the stiffness of the system and achieving part of swing using potential and stored energy. The second control strategy, "Var-S1" (see Figure 7-20 b)) intends to minimize the

wasted energy (no stored) at the pre-swing, by increasing the stiffness of the joint at that sub-phase, reducing the deflection of the joint allowed during the loading response.

Figure 7-21 presents the knee joint position and deflection resulting for implementing both strategies during different experiments, and compared with the system with fix compliance. During the loading response, both strategies allow deflection at the joint. A larger deflection occurs at the Var-S1, as a consequence of lower stiffness. During the holding position of the leading leg, 


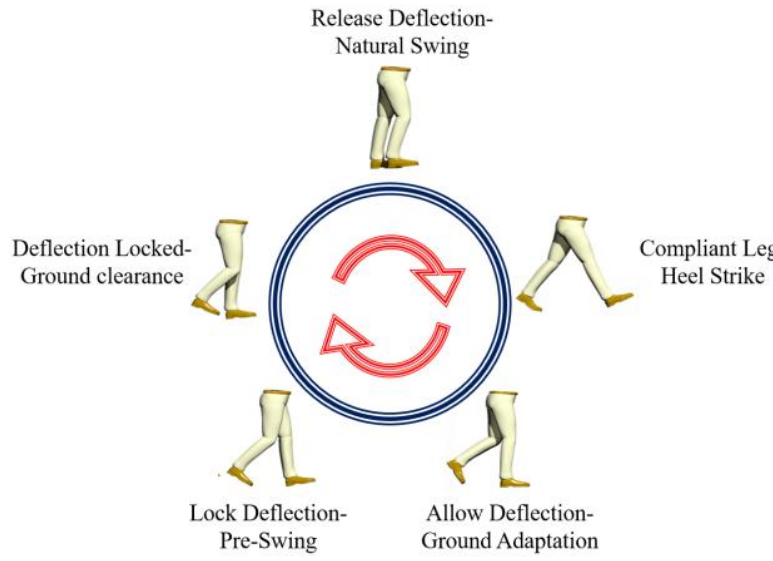

(a)

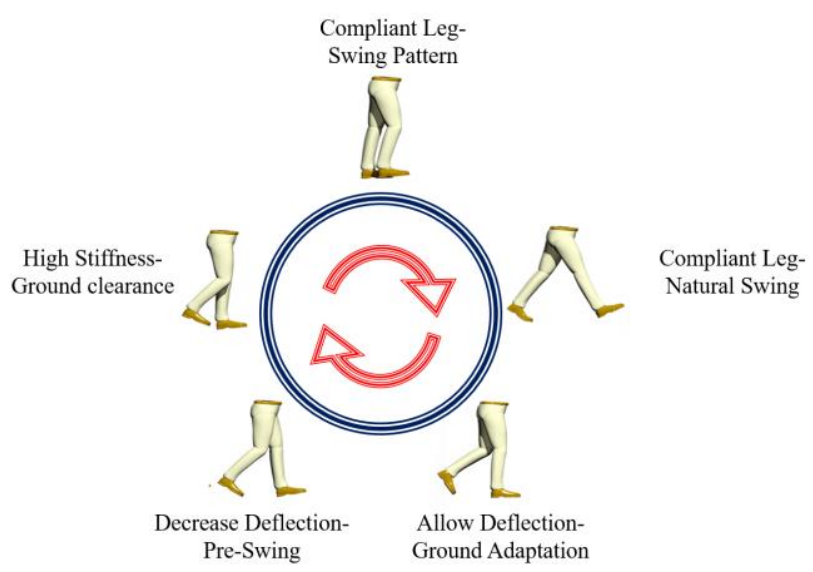

(b)

Figure 7-20. State Machine Commanded at the Knee. a) SM- for Lock Implementation, and b) SM- without Locking

followed by the pre-swing, in Var-L1, the stiffness of the joint is decreased to allow a larger deflection that can secure the engaging of the locking mechanism.

It can be noticed, when implemented the Var-S1, that a large deflection associated with the loading response is experienced at the knee. In the highlighted blue shaded area, the stiffness of the system is increased followed by a minimum deflection at the joint. When the foot leaves the ground at the beginning of the swing, the body weight is no-longer exerting load at the joint. The change of direction at the knee torque causes the elastic elements to act in the opposite direction. The previous deflection opposes to the flexion at the knee, thus a low deflection at the pre-swing is desired when implementing this strategy. The Var-S1 strategy seeks to provide the joint with adaptability at the loading response and support phase, and adjust to stiffer configurations when compliance won't be useful. This control strategy can be implemented with ARES and ARES-XL, larger deflection at the loading response can be achieved with ARES-XL.

The stiffness level at the loading response when implemented Var-L1 allowed some deflection for adaptation. During the holding position region, the stiffness is decreased to ensure a deflection larger than 10 degrees, where the locking can be engaged. The locking position can be adjusted to larger degree values, as can be seen in Figure 7-21 where the deflection reached values around 20 degrees. During the beginning of the swing, the deflection is constrained to side of the extension. It can be noticed, in the blue shaded area, that around 10 degrees the value is locked. 


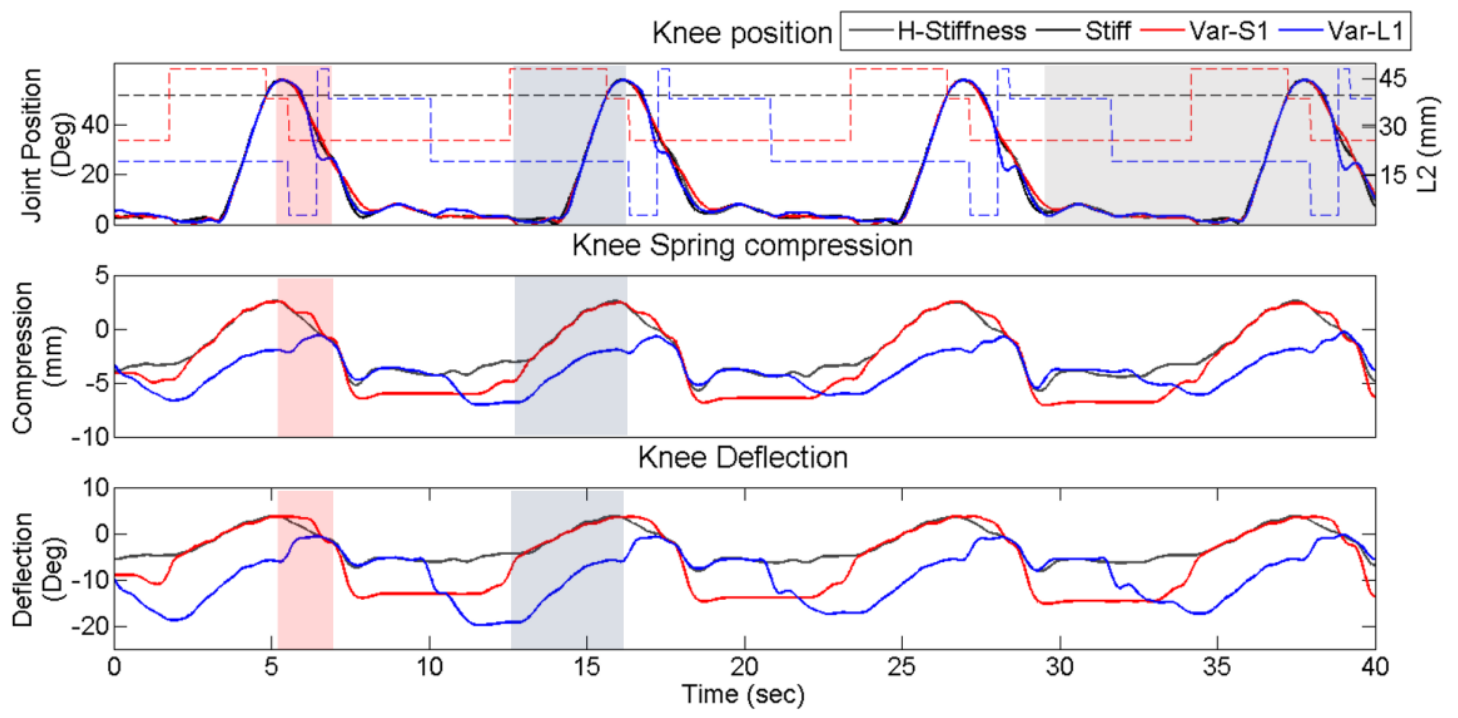

Figure 7-21. Knee Results with State Machines Implemented.

When the maximum knee flexion has been reached, the deflection from the support phase is stored and can be re-utilized for providing extension without the knee of the main motor -M1. The slider element is commanded to adjust $\mathrm{L}_{2}$ to a minimum value. The displacement of the element produces tension in the cable attached at the pawl-L1, causing the locking to disengaged. The reddish region in Figure 7-21 shows the moment when the locking is disengaged. The deflection at the knee drastically drops, producing extension motion in the leg swinging leg. The potential energy and inertia from the shank, along with the stored deflection, allow the joint to move like a free swing. The joint position shows that the swing extension occurs faster when implementing the Var-L1 scheme. The main motor $-\mathrm{M} 1$ could use the impulse giving by the free swing to continue the extension smoothly.

Figure 7-22 shows the velocity at the joint and the current consumed by the main motor M1 during the tests. A significant increase in the velocity can be noticed during the swing. The increment in the velocity occurs without alterations in the current of the main motor, evidence of being caused by the release of the deflection and the low stiffness commanded. The swing during the locomotion, should be achieved at a natural velocity given by the potential energy and the knee as free joint. Constraining the velocity of the swing, affects the balance when walking dynamically, the implementation of this feature could result beneficial in terms of stability and naturalness of the gait. 
Incrementing the walking speed, to achieve a self-selected velocity from the user still requires further research in terms of safety, and actuation power. Preliminary evaluation of implementing the Var-L1 strategy, shows that large adaptability can be provided during loading phase, with the ability of storing the energy of the pre-swing to further exploitation at the end of the swing. ARES-XL seems like an actuator that could deliver the required versatile functionality of the knee, while actuating based on the interaction of the user with the ground and reutilizing the absorbed power during the locomotion cycle.

\subsubsection{Ankle Implementation}

During the locomotion cycle, the ankle presents two main regions of power generation. As discussed in chapter 3, the dorsiflexion at the support phase accommodates to the ground reaction, storing energy that is released later at the push-off as an impulsive torque. ARES-XL was chosen to actuate the ankle at the compliant exoskeleton, because of its larger energy storing capabilities, when compared to ARES. In order to evaluate the behavior of the compliant joint, a State machine strategy was implemented to control the online adjustments of the stiffness in the system.

Figure 7-24 presents the diagram of the implemented control strategy. The stiffness of the joint is set to be highly compliant, allowing large deflection due to dorsiflexion while providing flexion torque. An increase of the stiffness in combination with a change in the rotation direction,

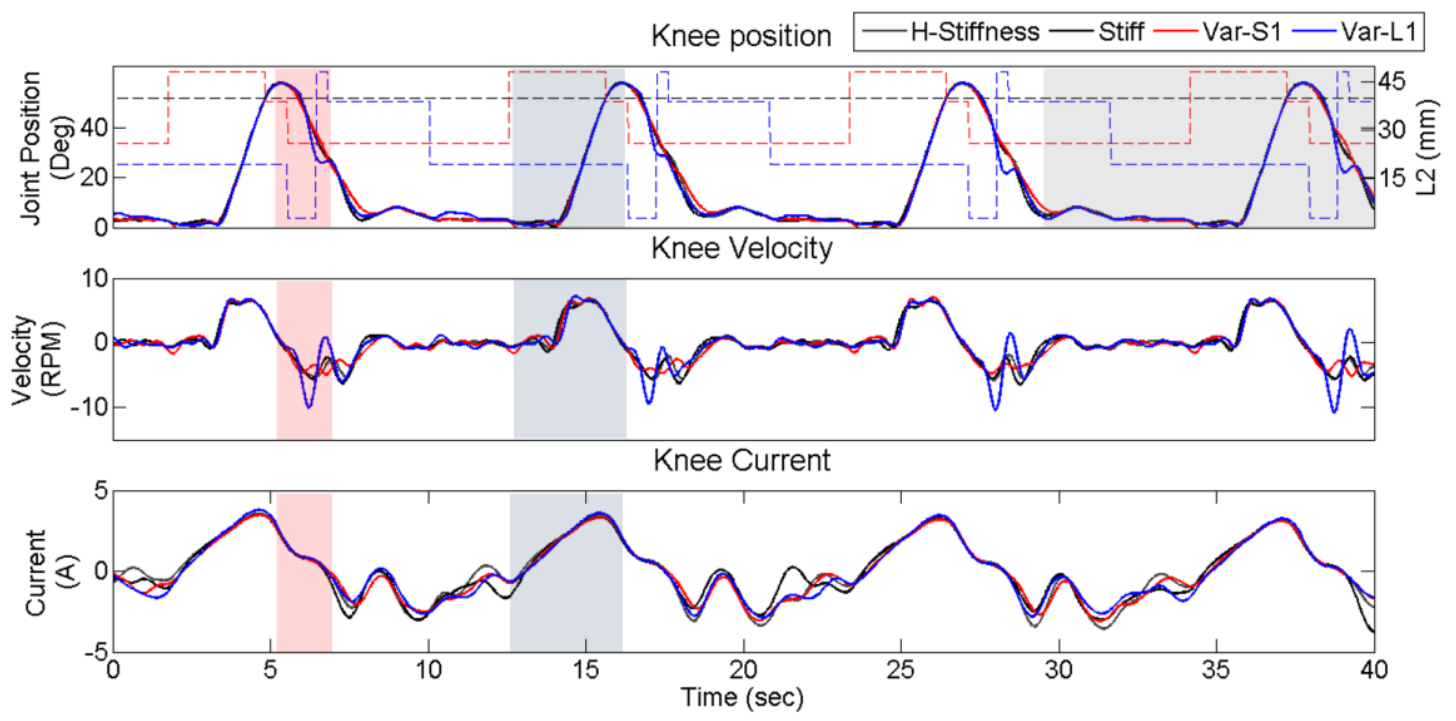

Figure 7-22. Knee Results with State Machines Implemented- Velocity and Current. 
should produce an impulsive torque, with high velocity as observed in the ankle experiments from chapter 6 .

The behavior of the joint is shown in Figure 7-24. During the loading response, the foot quickly gets loaded due to the reaction with the ground. The Torque generated by the joint is in the direction of the ankle flexion, while the rotation generates dorsiflexion. During this phase the foot is accommodated to the ground, and supports the body weight. Part of this load is transferred to the exoskeleton structure, and hold by the leg of the test dummy. As a consequence, the load at the actuator seems to be lower than expected from the biomechanical analysis. The highlighted reddish area in Figure 7-24, shows the stiffness adjustment that should contributes the impulse during the ankle flexion. At this point, the compliant mechanism should be loaded, and the stored deflection during the dorsiflexion should be at its maximum. From observation during the experiments, and as evidenced in the plots, no deflection is evidenced at the beginning of the flexion. The foot seems to be already in the air, and as a consequence no ground reaction occurs with it. Modifications of the system, or some adaptations in the cycle could modify the foot contact with the ground, making it last longer or forward the push-off in the ankle trajectory.

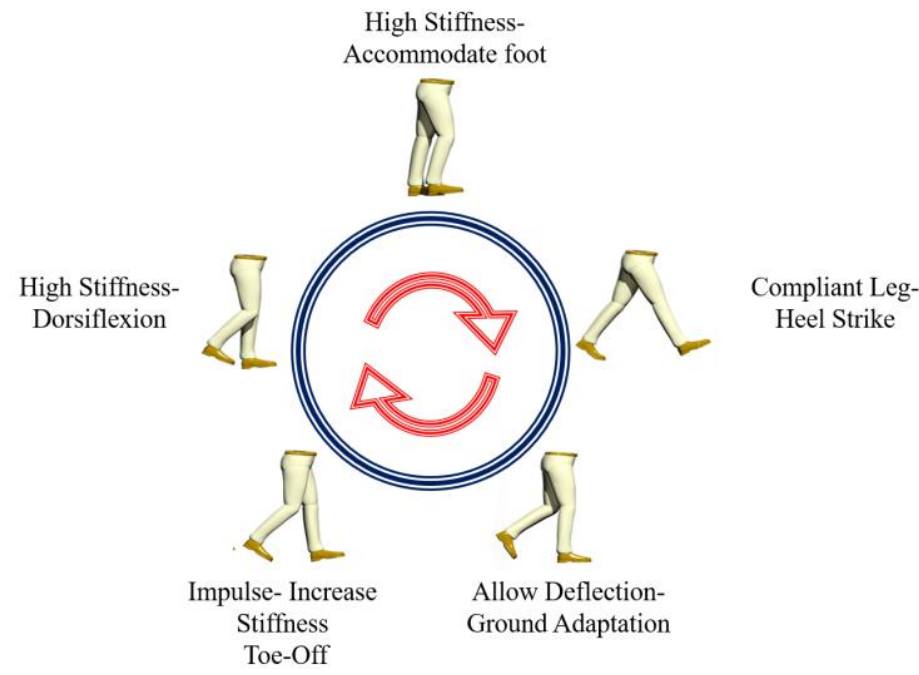

Figure 7-23. State Machine Commanded at the Ankle (Ankle-SM). 
The high stiffness is kept after the "push-off", to provide a quick arrangement of the foot and assist the ground clearance. Just before the heel strike, the compliance is adjusted once again to facilitate the contact with the ground.

Based on the results of this evaluation, some actuations alternative could be suitable for the ankle of the ATLAS prototype. The simplified version of ARES, presents a lighter mechanical structure with inherent compliant behavior that under the current torque behaviors seems like an adequate actuation for this joint. However, future adaptations of the gait or system's constrains will allow better exploitation of the ankle biomechanics and ARES-XL properties.

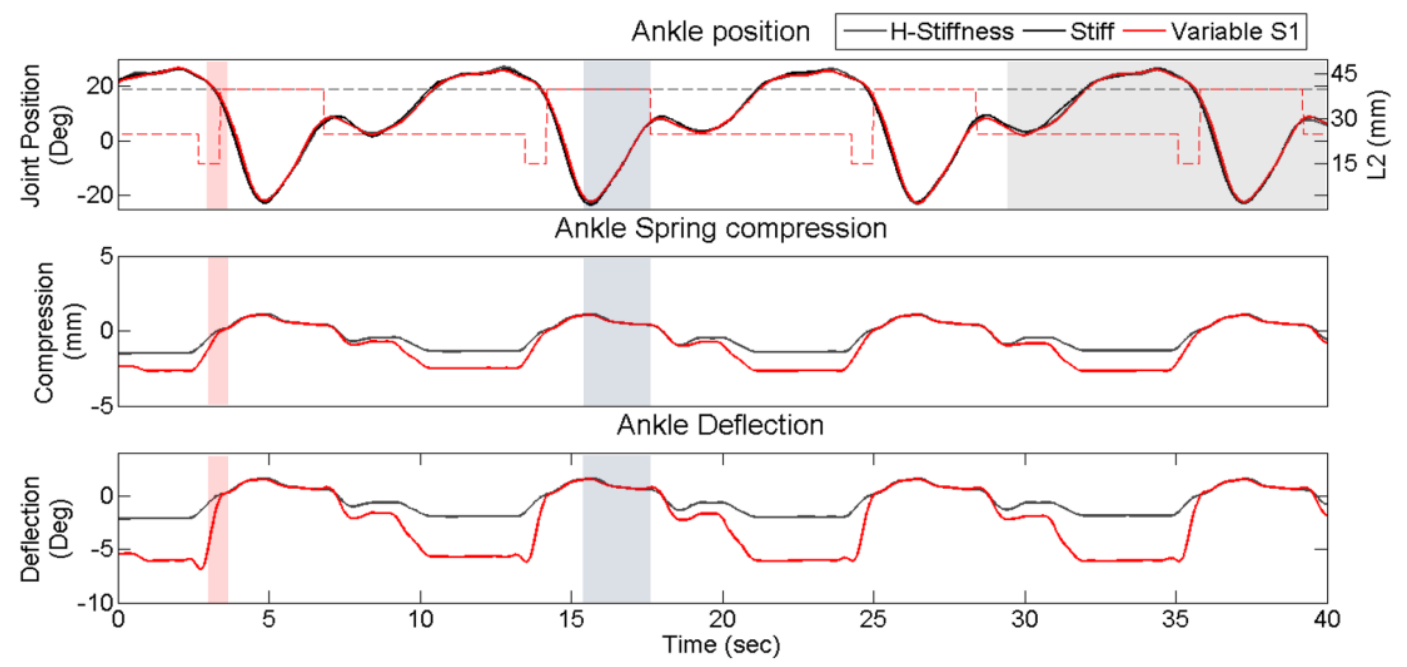

Figure 7-24. Position and Deflection when Implementing Ankle-SM.

The presented experiments represent the preliminary assessment of the first pediatric compliant exoskeleton, several interesting results can be observed from the data obtained. With the implementation of a simple position control, adaptability to the ground reactions, and freedom of movement can be obtained. These is caused by the implementation of inherent compliant systems to control the position of the exoskeleton's joints.

The compliant exoskeleton, not only brings benefits to the mechanical structure of the exoskeleton, by avoiding the impacts and shocks to be transmitted directly to the structure but absorbed with the elastic elements connected to it. But also, improve the user safety in case of rigidity on its joints. The compliant systems allow deviations from the actual commanded position to adapt to the user condition. 
The results after implementing the State Machines, in order to exploit the gait biomechanics, present interesting results that could be translated into significant energy savings. Adjusting the implemented control, in combination with the actuator mechanics the energy produced during gait can be efficiently stored and reutilize to decrease the motor power requirements. Table 7-2 presents the principle characteristics of the exoskeletons of the State of the Art and this novel compliant exoskeleton, with inherent safety characteristics and adaptability.

Table 7-2. Over-Ground gait Exoskeletons and ATLAS-C.

\begin{tabular}{|c|c|c|c|c|c|c|}
\hline $\begin{array}{c}\text { Exoskeleton } \\
\text { Device }\end{array}$ & $\begin{array}{c}\text { Weight } \\
(\mathbf{k g})\end{array}$ & Balance & $\begin{array}{l}\text { Users } \\
\text { hight } \\
\text { (cm) }\end{array}$ & $\begin{array}{c}\text { Actuated } \\
\text { DOF }\end{array}$ & Gait & Actuation \\
\hline $\operatorname{ReWalk}^{\mathrm{TM}}$ & 23.3 & $\begin{array}{l}\text { User-Cluthes or } \\
\text { walker }\end{array}$ & $\begin{array}{l}160-190 \\
\text { (Adults) }\end{array}$ & 2 per Leg & $\begin{array}{c}\text { Predefined-Fix } \\
\text { Based on healthy } \\
\text { subjects - NO ankle }\end{array}$ & $\begin{array}{c}\text { Stiff-Rigid } \\
\text { Compliant } \\
\text { Ankle }\end{array}$ \\
\hline Rex Bionics & 40 & Self-Balanced & $\begin{array}{l}160-190 \\
\text { (Adults) }\end{array}$ & 5 per leg & $\begin{array}{c}\text { Predefined-Fix } \\
\text { Addapted for static } \\
\text { stability }\end{array}$ & Stiff-Rigid \\
\hline Ekso Bionics & 20 & $\begin{array}{l}\text { User-Cluthes or } \\
\text { walker }\end{array}$ & $\begin{array}{l}158-188 \\
\text { (Adults) }\end{array}$ & 2 per Leg & $\begin{array}{c}\text { Predefined-Fix } \\
\text { Based on healthy } \\
\text { subjects - NO ankle }\end{array}$ & Stiff-Rigid \\
\hline $\begin{array}{c}\text { Indego } \\
\text { (Torque } \\
\text { Measurment) }\end{array}$ & 12 & $\begin{array}{l}\text { User-Cluthes or } \\
\text { walker }\end{array}$ & $\begin{array}{l}\text { 155-191 } \\
\text { (Adults) }\end{array}$ & 2 per Leg & $\begin{array}{c}\text { Predefined-Fix } \\
\text { Based on healthy } \\
\text { subjects - NO ankle }\end{array}$ & $\begin{array}{c}\text { Stiff-Rigid } \\
\text { Compliant } \\
\text { Ankle }\end{array}$ \\
\hline $\begin{array}{c}\text { NASA-X1 } \\
\text { (Torque } \\
\text { Measurment) }\end{array}$ & -- & $\begin{array}{l}\text { User-Cluthes or } \\
\text { walker }\end{array}$ & $\begin{array}{l}\text { 155-191 } \\
\text { (Adults) }\end{array}$ & 2 per Leg & $\begin{array}{c}\text { Predefined-Fix } \\
\text { Based on healthy } \\
\text { subjects - NO ankle }\end{array}$ & $\begin{array}{c}\text { Stiff-Rigid } \\
\text { Compliant } \\
\text { Ankle }\end{array}$ \\
\hline ATLAS & 10 & $\begin{array}{l}\text { User-Cluthes or } \\
\text { Walker } \\
\text { Self-Balance - } \\
\text { Special Walker }\end{array}$ & $\begin{array}{l}\text { 130-165 } \\
\text { (Children) }\end{array}$ & 3 per Leg & $\begin{array}{l}\text { Predefined-Fix } \\
\text { Based on healthy } \\
\text { subjects - WITH } \\
\text { ankle }\end{array}$ & Stiff-Rigid \\
\hline $\begin{array}{c}\text { ATLAS-C } \\
\text { (Torque } \\
\text { Measurement) }\end{array}$ & 11 & $\begin{array}{l}\text { User-Cluthes or } \\
\text { Walker } \\
\text { Self-Balance - } \\
\text { Special Walker }\end{array}$ & $\begin{array}{c}\text { 130-165 } \\
\text { (Children) }\end{array}$ & 3 per Leg & $\begin{array}{l}\text { Predefined-Inherent } \\
\text { adaptable } \\
\text { Based on healthy } \\
\text { subjects - WITH } \\
\text { ankle }\end{array}$ & Compliant \\
\hline
\end{tabular}




\section{Chapter 8}

\section{General Conclusions}

Walking is one of the most basic and common things in life, however it involves very complex mechanisms including energy storing, transfer and return which depend on a highly complex anatomical bone, muscle and tendon structure. During the locomotion cycle, continuous interaction between the ground and the feet occurs. Natural adaptation of joints during normal walking is a requirement that must be met by lower-limb robotic exoskeletons.

Many research groups have been working in the development of adjustable compliant actuators. However, as accepted by many researchers there is not perfect or optimal design. Actuators need to be designed oriented to the desired application and operation. Conversely, some common characteristics can be spotted on many designs, such as working principles for achieving stiffness adjustments, and compactness.

This $\mathrm{PhD}$ thesis developed two main actuation systems, such devices will provide the required actuation to the ATLAS exoskeleton, a robotic device to be worn by children. Size, and weight are significantly important in this particular application. Also, safety based on compliance. Both actuators were designed to be force-controlled, and compliant. Lateral size is optimized by taking advantage of the different elements included in the systems to achieve different tasks; such as the compliant behavior due to the elastic elements and their utilization for achieving a good torque measure.

The results from simulations and verified by the prototypes evaluations reveal that the adjustable rigidity is closely related to the torque measurement scale. By proper adjustment, the actuator can be controlled for different functions, such as rehabilitation or walking at different speeds. Different stiffness configuration can produce more or less deflection at the joint at a given

torque. Adaptability can be adjusted for different subjects based on the load transferred to the exoskeleton's joints. 
Considerable number of potential users of robotic exoskeletons suffer spasmodic movements and non-uniform joint rigidity, providing intrinsic compliance to their joints is absolutely necessary.

When compared side by side, ARES and ARES-XL present similar properties. The stiffness adjustment and torque measuring capabilities are based on the same principles, but their mechanical structures present different geometries, and couplings. Nevertheless, ARES-XL provide significant improvements in terms of maximum allowable deflection, and minimum output stiffness.

Based on the joint functionalities, when implementing the compliant actuators into the ATLAS exoskeleton, the hip joint could have been actuated by any of the designed actuators. However, to the author's understanding, simply by providing compliance and torque measuring capabilities to this joint, its behavior should be appropriate. With that in mind, an adaptation of ARES was implemented, removing the compliant frame, and setting the system to a fixed stiffness given by a manually adjusted distance.

The resulting system is intrinsically compliant, capable of measuring the torque exerted at the joint, and similar in weight and size to the stiff counterpart. During the walking assessment absorption of shocks was evidenced in the elastic elements. The joint followed the predefined trajectory allowing small deviations as consequence of ground reaction and inertial forces. Desired behaviors in the compliant exoskeleton, that contribute to smaller forces transmitted to the mechanical structure and adaptation properties.

Two control strategies were tested at the knee of the exoskeleton; by implementing 2 state machine-controllers ARES-XL was evaluated without adding the locking mechanism. The results obtained with this configuration could also be achieved with ARES. The goal of the experiment was to validate that tuning the joint stiffness can allow adaptability during loading response, larger adaptability/deflection could eventually translate in achieving the loading response passively. The joint behaving simply as rotational spring. From the experiments, is shown that during the loading response large deflections can occur in the leading leg. At the middle of the support phase, the stiffness can gradually increase, to reduce the deflection at the joint at the pre-swing. Otherwise, 
any deflection would produce motion in the opposite direction when the foot leaves the ground. This energy is wasted and can affect the ground clearance, thus increasing the stiffness on the system significantly reduces the deflection at that phase. Deflections of 10 degrees or more were experienced at the loading response, and by successfully adjusting the joint stiffness, at the preswing, the wasted deflection at the swing is smaller than three degrees.

The locking mechanism included in the ARES-XL actuator, proved to be successfully engaged in the loading response, blocking the deflection at a given position. When the locking control-strategy was implemented, the load exerted during the beginning of the swing to flex the knee, neither affect the previous deflection, nor causes the pawl $-\mathrm{L} 1$ to disengage. When the extension of the knee is commanded at the end-swing, the disengaging of the lock, combined with adjusting the stiffness to a very low value; causes the knee to behave as a free pendulum, partially extending the knee without effort and at a velocity given by the inertia of the body and its potential energy.

This capability was tested and proved in the single joint at the end of Chapter 6. Its implementation in the compliant exoskeleton, combined with a state machine showed similar results. The stored deflection can be increased, by adding tooth to the element in the main motor -M1, allowing different locking positions. From the experiment, at the pre-swing, the body weight can produce deflections at the knee up to 20 degrees. This deflection could later translate to 20 degrees of extension by means of potential energy at the end of the swing.

Taking advantage of the larger deflection allowed by ARES-XL, energy can be passively stored into the elastic elements during the approximate 20 degrees of dorsi-flexion in the ankle during the support phase. This energy can be reutilized during the push-off by quickly adjusting the stiffness to a higher level. As a consequence of the stiffness adjustment, the deflection will reduce transferring to the joint position as movement in the direction of the exerted torque. This transferred energy, combined with the remaining energy in the elastic elements, and the main motor $-\mathrm{M} 1$ actuation, an impulsive reaction is generated at the joint. During the single joint experiments, this hypothesis was tested by loading the system allowing some deflection in it. A step reference was commanded at the same time of the stiffness adjustment, resulting in a torque generation at speed rate significantly higher than the achieved with stiff configuration, or fixed compliance at the joint. 
The impulsive behavior achieved in the experiments in Chapter 6, represent a proof of concept that motivated the implementation of ARES-XL into the ankle of the compliant exoskeleton. However, the expected results during the walking assessment wasn't achieved. From the analysis of the data, it seems that the several constrains to achieve a walking- alike natural affect the foot reaction with the ground, and causes the foot to prematurely leave the ground.

This work presented the design, model, and preliminary evaluation of a compliant exoskeleton. The general results show that as expected from the literature, intrinsic compliance in the exoskeleton's joints translate in adaptability to the environment. ARES-XL exceeds ARES in compliant capabilities, but to exploit them in the exoskeleton, the implemented controller needs to take the ground reactions into consideration.

\subsection{Future Work}

After the preliminary assessment of the compliant exoskeleton, future work is planned to evaluate the compliant exoskeleton with healthy subjects. By tracking the joint torques during a predefined trajectory with high stiffness, the particular sections of loading response, pre-swing, push-off, and swing can be identified when using the exoskeleton. Based on the behavior of the joints when using the exoskeleton, special control strategies can be developed for the compliant ATLAS exoskeleton and its particular DOF. The developed control strategies can be implemented with states machine at knee and ankle joints to exploit the system properties and biomechanics of the gait.

Focusing in the size and weight optimization of the compliant actuation devices, more compact designs can be developed, and improvements on the locking mechanism can be achieved by adding a controllable Pawl-L1. The implementation of a servo motor to mechanically control the locking pawl $-\mathrm{L} 1$ could result in a more versatile actuator.

The locomotion cycle when using the exoskeleton is constrained by the device joints and the range of mobility allowed by the balance strategy. Incrementing the DOF in the walker frame, in order to improve the human-like gait could result in a more natural gait, with presence of pushoff and ankle adaptation to the ground. 


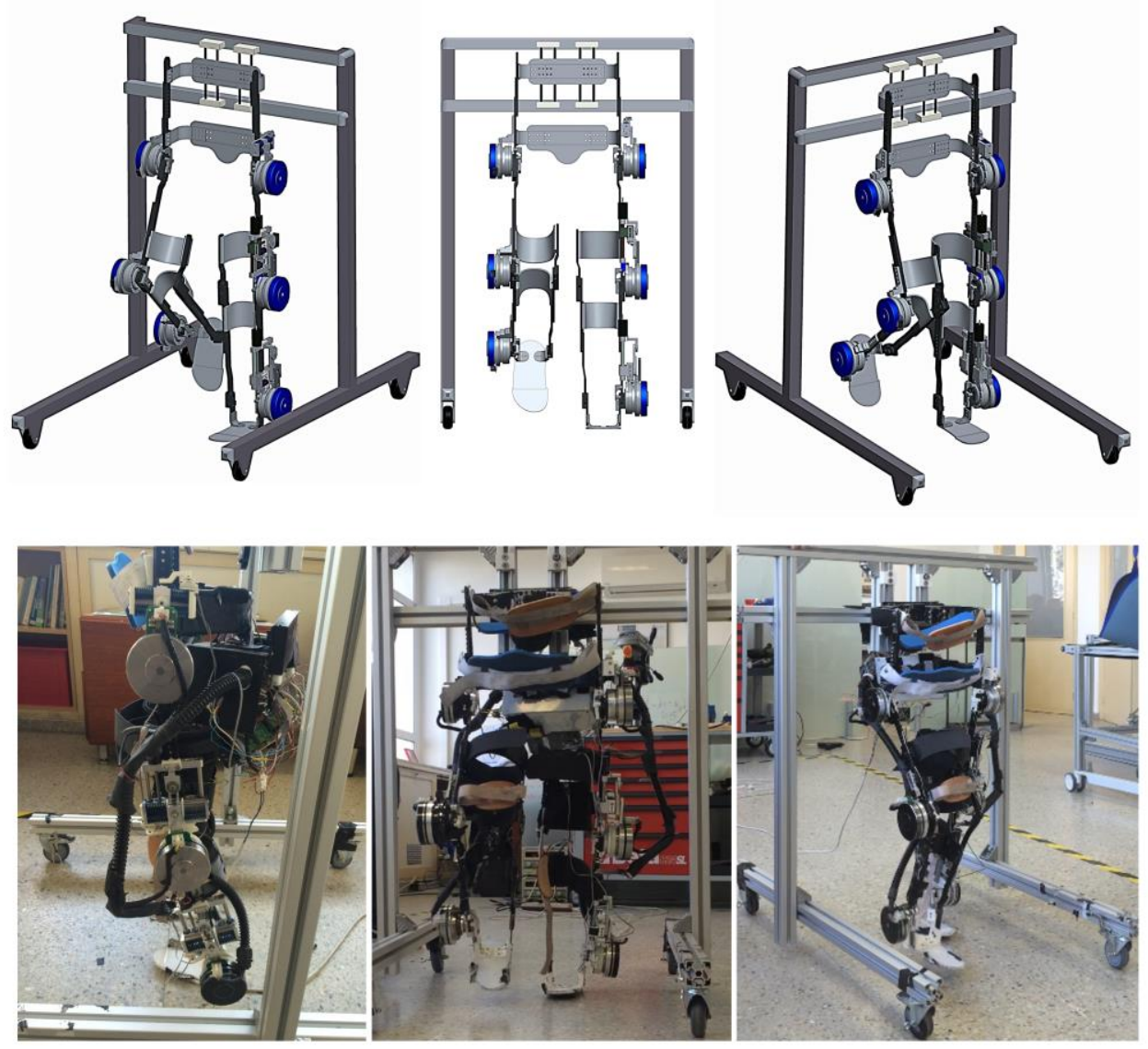

Figure 8-1. ATLAS- Compliant Prototype and CAD design.

\subsection{Publications and stays research}

During the development of this doctoral thesis, the author has collaborated with two internationally recognized research center during his stays research, and several scientific publications and patents have been produced.

\subsubsection{Stay Research}

- Jun to Nov/2014 UNIVERSITY OF HOUSTON - Houston, USA. Visitor Scholar at the "Laboratory for Noninvasive Brain-Machine Interface Systems". 
- Participation on tests with SCI subjects on board of the commercial exoskeleton REX, commanded by the detection of user intention by means of user's EEG signals.

- Off-line data analysis of the EEG and kinematic data collected during the experiments to detect the presence of motion or eye artifacts.

- Sep to Dec/2013 VRIJE UNIVERSITEIT BRUSSELS - Brussels, Belgium. Visitor Scholar at the "Robotics and Multibody Mechanics Research Group".

- Instrumentation of the AMP-Foot 3.0, a powered prosthetic ankle for amputees.

- Developed of basic control and evaluation of the prosthesis components.

\subsubsection{Journal publications}

- M. Cestari, D.Sanz-Merodio, J.C. Arevalo and E. Garcia. "An Adjustable Compliant Joint for Lower-Limb Exoskeletons". IEEE/ASME Transactions on Mechatronics (2015), Vol.20, no.2, pp.889-898.

- E. Garcia, J.C. Arevalo, M. Cestari and D.Sanz-Merodio. "On the technological instantiation of a biomimetic leg concept for agile locomotion". ASME Journal of Mechanism and Robotics (2015), Vol.7, no.3.

- M. Cestari, D.Sanz-Merodio, J.C. Arevalo and E. Garcia. "ARES, a variable stiffness actuator with embedded force sensor for the ATLAS exoskeleton". ndustrial Robot: An International Journal (2014), Vol. 41 Iss: 6, pp.518 - 526.

- D. Sanz-Merodio, M. Cestari, E. Garcia. "Generation and control of adaptative gaits in lower-limb exoskeletons for motion assistance", International Journal of Advanced Robotic Systems 2014, Vol 28, no.5, pp.329-338.

- J.C. Arevalo, M. Cestari, D.Sanz-Merodio and E. Garcia. "On the Necesity of Including Joint Passive Dynamics in the Impedance Control of Robotic Legs". International Journal of Advanced Robotic Systems 2014, DOI: 10.5772/58474.

- P. Cherelle, V .Grosu, M. Cestari, B. Vanderborght, and D. Lefeber. "The amp-foot 3- new generation propulsive prosthetic feet with explosive motion characteristicsdesign \&validation”. BioMedical Engineering OnLine (Submitted in 2014-Accepted). 
- D. Sanz-Merodio, M. Cestari, J. C. Arevalo, E. Garcia, (2013) "Exploiting joint synergy for actuation in a lowerlimb active orthosis", Industrial Robot: An International Journal, Vol. 40 Iss: 3, pp.224- 228.

- D. Sanz-Merodio, M. Cestari, J.C. Arevalo and E. Garcia. "Control Motion Approach of a Lower Limb Orthosis to Reduce Energy Consumption”. International Journal of Advanced Robotic Systems 2013, Vol 1, no.3, pp.219-233.

\subsubsection{Conference publications}

- M. Cestari, D.Sanz-Merodio, J.C. Arevalo and E. Garcia. "Actuator with AdjustableRigidity and Embedded Sensor for an Active Orthosis Knee Joint”. CLAWAR 2013. Nature-Inspired Mobile Robotics, pp. 59-67.

- D. Sanz-Merodio, M. Cestari, J.C. Arevalo and E. Garcia. "Implementation of an adjustable compliant knee joint in a lower-limb exoskeleton". CLAWAR 2013. NatureInspired Mobile Robotics, pp. 223-231.

- D. Sanz-Merodio, M. Cestari, J.C. Arevalo and E. Garcia. "Gait parameter adaptation for lower-limb exoskeletons". International Work-Conference on Bioinformatics and Biomedical Engineering 2013, pp. 667-675.

- D. Sanz-Merodio, M. Cestari, J.C. Arevalo and E. Garcia. "A lower-limb exoskeleton for gait assistance in quadriplegia". IEEE International Conference on Robotics and Biomimetics 2012, pp. 122-127.

- M. Cestari, D.Sanz-Merodio, J.C. Arevalo and E. Garcia. "Development of Synergic Multiarticular Actuation in a Lower-limb Active Orthosis". 13th Mechatronics Forum International Conference. 2012. Vol.1, pp. 34-39.

\subsubsection{Patents}

Three patents have been produced as consequence of this PhD work. They are currently under commercial exploitation by Marsi-Bionics.

- PCT/ES2015/070855. E. Garcia, M. Cestari, D.Sanz-Merodio, and X. Carrillo "Exoesqueleto para asistencia al movimiento humano" (11-25-2015) Assignee: CSIC$\operatorname{UPM}(85 \%)$, M-B (15\%). 
- ES201330882 \& WO2014198979 (Prosecution). M. Cestari, D.Sanz-Merodio, y E. Garcia "Articulation with controllable stiffness and force-measuring device" (06-132013). Licensed to M-B by the CSIC and UPM.

- ES201330882 - EP2907495 (Prosecution) \& US2015265490 (Prosecution). M. Cestari, D.Sanz-Merodio, J.C. Arevalo y E. Garcia. "Walker comprising mechanism for assisting a user in standing and sitting operations" (10-11-2012). Licensed to M-B by the CSIC and UPM. 


\section{References}

[1] A. Albu-Schaffer, O. Eiberger, M. Grebenstein, S. Haddadin, C. Ott, T. Wimbock, S. Wolf, and G. Hirzinger. Soft robotics. Robotics Automation Magazine, IEEE, 15 (3): 20 -30, september 2008. ISSN 1070-9932. doi: 10.1109/MRA.2008.927979.

[2] R. Alexander. Three uses of springs in legged locomotion. The International Journal of Robotics Research, 9: 53-61, 1990.

[3] ARGO Medical Technology Inc. ReWalk-tm, 2016. URL http://www.rewalk.com//.

[4] S. K. Au, H. Herr, J. Weber, and E. C. Martinez-Villalpando. Powered ankle-foot prosthesis for the improvement of amputee ambulation. Conference of the IEEE EMBS, 2007.

[5] S. K. Banala, S. K. Agrawal, and J. P. Scholz. Active leg exoskeleton (alex) for gait rehabilitation of motor-impaired patients. In Rehabilitation Robotics, 2007. ICORR 2007. IEEE 10th International Conference on, pages 401-407, June 2007. doi: 10.1109/ICORR.2007.4428456.

[6] S. K. Banala, S. K. Agrawal, S. H. Kim, and J. P. Scholz. Novel gait adaptation and neuromotor training results using an active leg exoskeleton. IEEE/ASME Transactions on Mechatronics, 15 (2): 216-225, April 2010. ISSN 1083-4435. doi: 10.1109/TMECH.2010.2041245.

[7] R. Bogue. Robotic exoskeletons: a review of recent progress. Industrial Robot: An International Journal, 42 (1): 5-10, 2015. doi: 10.1108/IR-08-2014-0379. URL http://dx.doi.org/10.1108/IR-08-2014-0379.

[8] M. Cestari, D. Sanz-Merodio, J. C. Arevalo, and E. Garcia. Andador con mecanismo de asistencia en operaciones de levantado y sentado de un usuario., 2014. 
[9] M. Cestari, D. Sanz-Merodio, J. C. Arevalo, and E. Garcia. An adjustable compliant joint for lower-limb exoskeletons. IEEE/ASME Transactions on Mechatronics, 20 (2): 889-898, April 2015. ISSN 1083-4435. doi: 10.1109/TMECH.2014.2324036.

[10] P. Cherelle, V. Grosu, P. Beyl, A. Mathys, R. Van Ham, M. Van Damme, B. Vanderborght, and D. Lefeber. The maccepa actuation system as torque actuator in the gait rehabilitation robot altacro. In Biomedical Robotics and Biomechatronics (BioRob), 2010 3rd IEEE RAS and EMBS International Conference on, pages 27-32, 2010. doi: 10.1109/BIOROB.2010.5627030.

[11] J. L. Contreras-Vidal and R. G. Grossman. Neurorex: A clinical neural interface roadmap for eeg-based brain machine interfaces to a lower body robotic exoskeleton. In Engineering in Medicine and Biology Society (EMBC), 2013 35th Annual International Conference of the IEEE, pages 1579-1582, July 2013. doi: 10.1109/EMBC.2013.6609816.

[12] A. M. Dollar and H. Herr. Lower extremity exoskeletons and active orthoses: Challenges and state-of-the-art. IEEE Transactions on Robotics, 24 (1): 144-158, FEB 2008. ISSN 1552-3098. doi: 10.1109/TRO.2008.915453.

[13] O. Eiberger, S. Haddadin, M. Weis, A. Albu-Scha?ffer, and G. Hirzinger. On joint design with intrinsic variable compliance: derivation of the dlr qa-joint. In Robotics and Automation (ICRA), 2010 IEEE International Conference on, pages 1687 -1694, may 2010. doi: 10.1109/ROBOT.2010.5509662.

[14] Ekso Bionics. Ekso, 2016. URL http://www.eksobionics.com/.

[15] C. English and D. Russell. Mechanics and stiffness limitations of a variable stiffness actuator for use in prosthetic limbs. Mechanism and Machine Theory, 34 (1): 7-25, JAN 1999. ISSN 0094-114X. doi: 10.1016/S0094-114X(98)00026-3.

[16] R. J. Farris, H. A. Quintero, S. A. Murray, K. H. Ha, C. Hartigan, and M. Goldfarb. A preliminary assessment of legged mobility provided by a lower limb exoskeleton for persons with paraplegia. IEEE Transactions on Neural Systems and Rehabilitation Engineering, 22 (3): 482-490, May 2014. ISSN 1534-4320. doi: 10.1109/TNSRE.2013.2268320. 
[17] Federación Española de Enfermedades Neuromusculares. ASEM, 2016. URL http://www.asem-esp.org/.

[18] M. Frey, G. Colombo, M. Vaglio, R. Bucher, M. Jorg, and R. Riener. A novel mechatronic body weight support system. IEEE Transactions on Neural Systems and Rehabilitation Engineering, 14 (3): 311-321, Sept 2006. ISSN 1534-4320. doi: 10.1109/TNSRE.2006.881556.

[19] E. Garcia, D. Sanz-Merodio, F. Sanchez, J. Arevalo, and P. Gonzalez-de-Santos. Development of the atlas lower-limb active orthosis. In International Conference on Climbing and Walking Robots (CLAWAR), 2011. doi: 10.1142/9789814374286_0116.

[20] E. Garcia, M. Cestari, and D. Sanz-Merodio. Wearable exoskeletons for the physical treatment of children with quadriparesis. In Humanoid Robots (Humanoids), 2014 14th IEEE-RAS International Conference on, pages 425-430, Nov 2014. doi: 10.1109/HUMANOIDS.2014.7041395.

[21] D. Haeufle, M. Günther, R. Blickhan, and S. Schmitt. Can quick release experiments reveal the muscle structure? a bionic approach. Journal of Bionic Engineering, 9 (2): 211 - 223, 2012. ISSN 1672-6529. doi: http://dx.doi.org/10.1016/S1672-6529(11)60115-7. URL http://www.sciencedirect.com/science/article/pii/S1672652911601157.

[22] R. Ham, T. Sugar, B. Vanderborght, K. Hollander, and D. Lefeber. Compliant actuator designs. Robotics Automation Magazine, IEEE, 16 (3): 81 -94, september 2009. ISSN 1070-9932. doi: 10.1109/MRA.2009.933629.

[23] Y. Han and X. Wang. The biomechanical study of lower limb during human walking. Science China Technological Sciences, 54 (4): 983-991, 2011. ISSN 1862-281X. doi: 10.1007/s11431-011-4318-z. URL http://dx.doi.org/10.1007/s11431-011-4318-z.

[24] J. Hurst and A. Rizzi. Series compliance for an efficient running gait. Robotics Automation Magazine, IEEE, 15 (3): 42 -51, september 2008. ISSN 1070-9932. doi: 10.1109/MRA.2008.927693.

[25] J. Hurst, J. Chestnutt, and A. Rizzi. An actuator with physically variable stiffness for highly dynamic legged locomotion. In Robotics and Automation, 2004. Proceedings. ICRA 
'04. 2004 IEEE International Conference on, volume 5, pages 4662-4667 Vol.5, 2004. doi: 10.1109/ROBOT.2004.1302453.

[26] M. Hutter, C. Remy, M. Hoepflinger, and R. Siegwart. High compliant series elastic actuation for the robotic leg scarleth. In International Conference on Climbing and Walking Robots (CLAWAR), 2011.

[27] M. Hutter, a. M. B. C. Gehring, M. Hoespflinger, C. D. Remy, and R. Siegwart. Starleth: A compliant quadrupedal robot for fast, efficient, and versatile locomotion. In International Conference on Climbing and Walking Robots (CLAWAR), 2012.

[28] J. J. G. Iñaki Díaz and E. Sánchez. Lower-Limb Robotic Rehabilitation: Literature Review and Challenges. Journal of Robotics, 2011: 11, 2011.

[29] IHMC. X1-IHMC Exoskeleton, 2016. URL www.robots.ihmc.us.

[30] A. Jafari, N. Tsagarakis, and D. Caldwell. AwAS-II: A new Actuator with Adjustable Stiffness based on the novel principle of adaptable pivot point and variable lever ratio. In Robotics and Automation (ICRA), 2011 IEEE International Conference on, pages 4638-4643, 2011. doi: 10.1109/ICRA.2011.5979994.

[31] A. Jafari, N. Tsagarakis, and D. Caldwell. A Novel Intrinsically Energy Efficient Actuator With Adjustable Stiffness (AwAS). Mechatronics, IEEE/ASME Transactions on, 18 (1): 355-365, 2013. ISSN 1083-4435. doi: 10.1109/TMECH.2011.2177098.

[32] H. K. Kwa, J. Noorden, M. Missel, T. Craig, J. Pratt, and P. Neuhaus. Development of the IHMC mobility assist exoskeleton. In Robotics and Automation, 2009. ICRA '09. IEEE International Conference on, pages 2556-2562, 2009. doi: 10.1109/ROBOT.2009.5152394.

[33] La Federación Internacional de Espina Bífida e Hidrocefalia. Spina bifida- FEBHI, 2016. URL http://www.febhi.org/.

[34] K. Low. Robot-assisted gait rehabilitation: From exoskeletons to gait systems. In Defense Science Research Conference and Expo (DSR), 2011, pages 1-10, 2011. doi: 10.1109/DSR.2011.6026886. 
[35] M. F. M. CUERPO Y CORPORALIDAD EN LA PARAPLEJIA: UNA TEORÍA DE ENFERMERÍA. PhD thesis, Universidad Nacional de Colombia- Facultad de Enfermería, 2011.

[36] Marsi-Bionics. M-B, 2016. URL www.marsibionics.com.

[37] S. Migliore. Control of robotics joint using principles from the equilibrium point hypothesis of animal motor control. $\mathrm{PhD}$ thesis, Georgia institute of Technology, 2004.

[38] S. Migliore, E. Brown, and S. DeWeerth. Biologically inspired joint stiffness control. In IEEE International Conference on Robotics and Automation (ICRA), Vols 1-4, pages 4508-4513. IEEE, 2005. ISBN 0-7803-8914-X.

[39] D. S. Migliore S.A, Brown E.A. Novel Nonlinear Elastic Actuators for Passively Controlling Robotic Joint Compliance. ASME Journal of Mechanical Design, 129 (4): 406-412, 2006. doi: $10.1115 / 1.2429699$.

[40] P. D. Neuhaus, J. H. Noorden, T. J. Craig, T. Torres, J. Kirschbaum, and J. E. Pratt. Design and Evaluation of Mina a Robotic Orthosis for Paraplegics. In IEEE International Conference on rehabilitation Robotics (ICORR), International Conference on Rehabilitation Robotics ICORR, 2011. ISBN 978-1-4244-9862-8.

[41] U. of Oregon, 2015. URL http://mime.oregonstate.edu/.

[42] Parker. Indego Exoskeleton, 2016. URL www.Indego.com.

[43] M. Plooij, G. Mathijssen, P. Cherelle, D. Lefeber, and B. Vanderborght. Lock your robot: A review of locking devices in robotics. IEEE Robotics Automation Magazine, 22 (1): 106117, March 2015. ISSN 1070-9932. doi: 10.1109/MRA.2014.2381368.

[44] G. Pratt and M. Williamson. Series elastic actuators. In Intelligent Robots and Systems 95. 'Human Robot Interaction and Cooperative Robots', Proceedings. 1995 IEEE/RSJ International Conference on, volume 1, pages 399-406 vol.1, 1995. doi: 10.1109/IROS.1995.525827.

[45] S. M. M. Rahman and R. Ikeura. A novel variable impedance compact compliant ankle robot for overground gait rehabilitation and assistance. Procedia Engineering, 41 (0): 522 - 
531, 2012. ISSN 1877-7058. doi: 10.1016/j.proeng.2012.07.207. URL http://www.sciencedirect.com/science/article/pii/S1877705812026070.

[46] REX Bionics. Rex, 2016. URL http://www.rexbionics.com/.

[47] D. Robinson, J. Pratt, D. Paluska, and G. Pratt. Series elastic actuator development for a biomimetic walking robot. In Advanced Intelligent Mechatronics, 1999. Proceedings. 1999 IEEE/ASME International Conference on, pages 561 -568, 1999. doi: 10.1109/AIM.1999.803231.

[48] D. W. Robinson. Design and Analysis of Series Elasticity in Closed-loop Actuator Force Control. PhD thesis, Massachusetts Institute of Technology, 1996.

[49] P. Rosenbaum, N. Paneth, A. Leviton, M. G. M. Bax, D. Damiano, B. Dan, and B. Jacobsson. A report: the definition and classification of cerebral palsy April 2006. Developmental medicine and child neurology, 109: 8-14, 2007.

[50] D. Sanz-Merodio, M. Cestari, J. C. Arevalo, and E. Garcia. A lower-limb exoskeleton for gait assistance in quadriplegia. In Robotics and Biomimetics (ROBIO), 2012 IEEE International Conference on, pages 122-127, Dec 2012. doi: 10.1109/ROBIO.2012.6490954.

[51] D. Sanz-Merodio, M. Cestari, J. C. Arevalo, and E. Garcia. Control motion approach of a lower limb orthosis to reduce energy consumption. International Journal of Advanced Robotic Systems, 9, 2012.

[52] R. Schiavi, G. Grioli, S. Sen, and A. Bicchi. Vsa-ii: a novel prototype of variable stiffness actuator for safe and performing robots interacting with humans. In Robotics and Automation, 2008. ICRA 2008. IEEE International Conference on, pages 2171 -2176, may 2008. doi: 10.1109/ROBOT.2008.4543528.

[53] B. Stansfield, S. Hillman, M. Hazlewood, and J. Robb. Regression analysis of gait parameters with speed in normal children walking at self-selected speeds. Gait \& Posture, 23 (3): 288 - 294, 2006. ISSN 0966-6362. doi: http://dx.doi.org/10.1016/j.gaitpost.2005.03.005. URL http://www.sciencedirect.com/science/article/pii/S0966636205000585.

[54] Statistics-Spain. $\quad$ Statistics, $2016 . \quad$ URL http://www.observatoriodeladiscapacidad.es/. 
[55] Statistics-Spinal cord injuries. SCI. URL www.nscusc.uab.edu.

[56] G. Tonietti, R. Schiavi, and A. Bicchi. Design and control of a variable stiffness actuator for safe and fast physical human/robot interaction. In Robotics and Automation, 2005. ICRA 2005. Proceedings of the 2005 IEEE International Conference on, pages 526-531, 2005. doi: 10.1109/ROBOT.2005.1570172.

[57] N. G. Tsagarakis, I. Sardellitti, and D. G. Caldwell. A new variable stiffness actuator (compact-vsa): Design and modelling. In Intelligent Robots and Systems (IROS), 2011 IEEE/RSJ International Conference on, pages $378-383$, sept. 2011. doi: 10.1109/IROS.2011.6095006.

[58] H. Vallery, J. Veneman, E. van Asseldonk, R. Ekkelenkamp, M. Buss, and H. van der Kooij. Compliant actuation of rehabilitation robots. Robotics Automation Magazine, IEEE, 15 (3): 60 -69, september 2008. ISSN 1070-9932. doi: 10.1109/MRA.2008.927689.

[59] B. Vanderborght, B. Verrelst, R. V. Ham, M. V. Damme, P. Beyl, and D. Lefeber. Development of a compliance controller to reduce energy consumption for bipedal robots. Autonomous Robots, 24: 41-434, 2008.

[60] B. Vanderborght, N. Tsagarakis, C. Semini, R. Van Ham, and D. Caldwell. MACCEPA 2.0: Adjustable compliant actuator with stiffening characteristic for energy efficient hopping. In Robotics and Automation, 2009. ICRA '09. IEEE International Conference on, pages 544 -549, may 2009. doi: 10.1109/ROBOT.2009.5152204.

[61] B. Vanderborght, A. Albu-Schaeffer, A. Bicchi, E. Burdet, D. Caldwell, R. Carloni, M. Catalano, O. Eiberger, W. Friedl, G. Ganesh, M. Garabini, M. Grebenstein, G. Grioli, S. Haddadin, H. Hoppner, A. Jafari, M. Laffranchi, D. Lefeber, F. Petit, S. Stramigioli, N. Tsagarakis, M. V. Damme, R. V. Ham, L. Visser, and S. Wolf. Variable impedance actuators: A review. Robotics and Autonomous Systems, 61 (12): 1601 - 1614, 2013. ISSN 0921-8890. doi: http://dx.doi.org/10.1016/j.robot.2013.06.009. URL http://www.sciencedirect.com/science/article/pii/S0921889013001188.

[62] B. Vanderborght, A. Albu-Schaeffer, A. Bicchi, E. Burdet, D. Caldwell, R. Carloni, M. Catalano, O. Eiberger, W. Friedl, G. Ganesh, M. Garabini, M. Grebenstein, 
G. Grioli, S. Haddadin, H. Hoppner, A. Jafari, M. Laffranchi, D. Lefeber, F. Petit, S. Stramigioli, N. Tsagarakis, M. V. Damme, R. V. Ham, L. Visser, and S. Wolf. Variable impedance actuators: A review . Robotics and Autonomous Systems, 61 (12): 1601 - 1614, 2013. ISSN 0921-8890. doi: http://dx.doi.org/10.1016/j.robot.2013.06.009. URL http://www.sciencedirect.com/science/article/pii/S0921889013001188.

[63] J. F. Veneman, R. Ekkelenkamp, R. Kruidhof, and H. v. d. K. F. C.T. van der Helm. A series elastic- and bowden-cable-based actuation system for use as torque actuator in exoskeleton-type robots. The International Journal of Robotics Research, 25: 261-281, 2006.

[64] VIACTORS consortium. VIACTORS, 2016. URL http://www.viactors.org.

[65] L. C. Visser, R. Carloni, F. Klijnstra, and S. Stramigioli. A prototype of a novel energy efficient variable stiffness actuator. In 2010 Annual International Conference of the IEEE Engineering in Medicine and Biology, pages 3703-3706, Aug 2010. doi: 10.1109/IEMBS.2010.5627424.

[66] Vrije Universiteit Brussel. Altacro:a Step Rehabilitation Robot, 2016. URL www.altacro.vub.ac.be.

[67] M. Vukobratovic, B. Borovac, D.Surla, and D. Stokic. Biped locomotion: Dynamics, stability, control and application. Springer, 1990.

[68] S. Wang, W. van Dijk, and H. van der Kooij. Spring uses in exoskeleton actuation design. In Rehabilitation Robotics (ICORR), 2011 IEEE International Conference on, pages 1-6, 29 2011-july 1 2011. doi: 10.1109/ICORR.2011.5975471.

[69] D. A. Winter and D. G. E. Robertson. Joint torque and energy patterns in normal gait. Biological Cybernetics, 29: 137-142, 1978.

[70] S. Wolf and G. Hirzinger. A new variable stiffness design: Matching requirements of the next robot generation. In IEEE Interantional Conference on Robotics and automation, pages 1741-1746. IEEE, 2008. ISBN 978-1-4244-1646-2. doi: 10.1109/ROBOT.2008.4543452. IEEE International Conference on Robotics and Automation, Pasadena, CA, MAY 19-23, 2008.

[71] D. world statistics. Disable world-statistics, 2016. URL http://www.disabledworld.com/. 
[72] H. Yu, S. Huang, G. Chen, S.-L. Toh, M. Cruz, Y. Ghorbel, C. Zhu, and Y. Yin. Design and analysis of a novel compact compliant actuator with variable impedance. In Robotics and Biomimetics (ROBIO), 2012 IEEE International Conference on, pages 1188-1193, 2012. doi: 10.1109/ROBIO.2012.6491131. 